\title{
Solenoidal Fields for Ion Beam Transport and Focusing
}

\author{
Edward Lee and Matthaeus Leitner \\ Lawrence Berkeley National Laboratory \\ August 2009
}

\section{Introduction and Contents}

In this report we calculate time-independent fields of solenoidal magnets that are suitable for ion beam transport and focusing.

There are many excellent Electricity and Magnetism textbooks that present the formalism for magnetic field calculations and apply it to simple geometries [1-1], but they do not include enough relevant detail to be used for designing a charged particle transport system. This requires accurate estimates of fringe field aberrations, misaligned and tilted fields, peak fields in wire coils and iron, external fields, and more. Specialized books on magnet design, technology, and numerical computations [1-2] provide such information, and some of that is presented here. The AIP Conference Proceedings of the US Particle Accelerator Schools [1-3] contain extensive discussions of design and technology of magnets for ion beams - except for solenoids. This lack may be due to the fact that solenoids have been used primarily to transport and focus particles of relatively low momenta, e.g. electrons of less than $50 \mathrm{MeV}$ and protons or $\mathrm{H}^{-}$of less than $1.0 \mathrm{MeV}$, although this situation may be changing with the commercial availability of superconducting solenoids with up to 20T bore field [1-4]. Internal reports from federal laboratories and industry treat solenoid design in detail for specific applications. The present report is intended to be a resource for the design of ion beam drivers for Inertial Fusion Energy [1-5] and Warm Dense Matter experiments [1-6], although it should also be useful for a broader range of applications.

The field produced by specified currents and material magnetization can always be evaluated by solving Maxwell's equations numerically, but it is also desirable to have reasonably accurate, simple formulas for conceptual system design and fast-running beam dynamics codes, as well as for general understanding. Most of this report is devoted to such formulas, but an introduction to the $\operatorname{Tosca}^{\odot}$ code [1-7] and some numerical results obtained with it are also presented. Details of design, fabrication, installation, and operation of magnet systems are not included; here we are concerned with calculations that precede or supplement detailed design. Mathematical derivations are presented with only a moderate number of steps. While there is no claim of originality, except for various numerical approximations and a conceptual induction module design in section 20 , many of the results and discussions are not readily available elsewhere.

Our primary topic is axisymmetric solenoidal systems with no magnetic materials. These simplifying features allow useful analytical calculations, which occupy sections 213. Deviations from axisymmetry are considered in sections 14, 15, 21, 22, and 23 and the effects of magnetic materials are treated in sections 16-20. Since magnetic aberrations 
are mixed with geometric aberrations in computing ion orbits, section 22 on the ion equations of motion in an arbitrary field is included.

\section{Contents}

Section 1. Introduction and Contents 1

\begin{tabular}{lll} 
Section 2. Solenoid Basics & 5 \\
\hline
\end{tabular}

2.1 Model assumptions and equations $\quad 5$

2.2 Simple field estimate $\quad 7$

2.3 Current distributions, global picture, and moments 8

2.4 Vector potential and flux lines 11

2.5 Stress and energy 12

\begin{tabular}{lr} 
Section 3. Near-Axis Field & 13 \\
\hline
\end{tabular}

3.1 On-axis field and near-axis expansion 13

3.2 Useful integrals 16

\begin{tabular}{ll} 
Section 4. Design Formulas for the On-Axis Field & 17 \\
\hline
\end{tabular}

4.1 Simple cases 17

4.2 Thick current layer $\quad 20$

4.3 Uniform fields 20

\begin{tabular}{ll} 
Section 5. Vector and Scalar Potentials & 23 \\
\hline
\end{tabular}

5.1 Vector potential 23

5.2 Magnetic scalar potential 24

5.3 Global scalar potential 25

\begin{tabular}{ll} 
Section 6. Green Function Solutions & 26 \\
\hline
\end{tabular}

6.1 Green function formalism 26

6.2 Explicit field expressions 27

6.3 Reduction to complete elliptic integrals 29

Section 7. Approximation of the Green Function Integrals $\quad 31$

7.1 Expansion of $f_{i}(a)$ in powers of $a \quad 31$

7.2 Approximation of $f_{i}(a)$ as $a \rightarrow 1$

7.3 Accurate approximation for $0 \leq a \leq 1$

7.4 Systematic approximation of $f_{i}(a) \quad 37$

Section 8. Thin Current Layer-Complete Solution 39

8.1Semi-infinite layer: near-axis fields 39

8.2 Semi-infinite layer: exact fields and potentials $\quad 40$

8.3 Current layer end divergence $\quad 42$

8.4 Finite length lens 43

8.5 Stored energy 45

8.6 Current ring 47

8.7 Approximation of fields and potentials

\begin{tabular}{lr} 
Section 9. Thick Current Layer & 54 \\
\hline
\end{tabular}

9.1 Uniform thick layer $\quad 54$

9.2 Approximation by a thin layer $\quad 57$

9.3 General thick layer - Bessel transform solution $\quad 57$ 
9.4 Pancake current layer $\quad 60$

\begin{tabular}{lll} 
Section 10. & Periodic Thin Current Layer & 61 \\
\hline
\end{tabular}

\begin{tabular}{lll} 
Section 11. & Far Field and External Field & 64 \\
\hline
\end{tabular}

11.1 Far field expansion 64

11.2 External field expansion $\quad 67$

\begin{tabular}{lll} 
Section 12. & Field in the Wire Layer & 69 \\
\hline
\end{tabular}

Section 13. Current Distribution to Produce a Given Field 71

\begin{tabular}{lll} 
Section 14. Misaligned and Tilted Solenoid & 74 \\
\hline
\end{tabular}

\begin{tabular}{lll}
\hline Section 15. Multiple Channels - Cross Talk & 76 \\
\hline
\end{tabular}

\begin{tabular}{lll} 
Section 16. & Magnetic Materials Basics & 78 \\
\hline
\end{tabular}

16.1 Ferromagnetism $\quad 78$

16.2 Magnetization formalism $\quad 80$

16.3 B-H relations and jump conditions $\quad 82$

16.4 Magnetization curve $\quad 85$

16.5 Permanent magnet solenoid $\quad 87$

Section 17. Wire Layer Embedded in a Highly Permeable Yoke 89

Section 18. Periodic Thin Current Layer with a Highly Permeable Yoke 93

\begin{tabular}{lll}
\hline Section 19. & Tosca $^{\odot}$ Model & 98 \\
\hline
\end{tabular}

19.1 General solution strategy in 3D $\quad 99$

19.2 Solution strategy in 2D 102

19.3 Example 103

Section 20. Application of Tosca ${ }^{\odot}$ to Induction Linac Design 107

20.1 Introduction 107

20.2 Example $\quad 110$

\begin{tabular}{lr} 
Section 21. Biot-Savart Formula & 120 \\
\hline
\end{tabular}

21.1 General formula 121

21.2 Straight wire segment field $\quad 122$

21.3 Helix field 123

Section 22. Single Particle Equations of Motion in an Arbitrary field 127

22.1 Time as the independent variable 127

22.2 Longitudinal distance as the independent variable $\quad 128$

22.3 Paraxial approximation $\quad 130$

\begin{tabular}{lr} 
Section 23. Steering Dipole Field & 130 \\
\hline
\end{tabular}

\section{References}

1-1 Jackson, J.D., Classical Electrodynamics, $2^{\text {nd }}$ or $3^{\text {rd }}$ edition, chap. 5, Wiley, New York (1975 or 1999).

Feynman, R., R. Leighton, and M. Sands, The Feynman Lectures on Physics, Vol. II, chaps. 13,14,15,36,37, Addison-Wesley, Reading, MA (1964).

Panofsky, W.K.H., and M. Phillips, Classical Electricity and Magnetism, $2^{\text {nd }}$ edition, chaps. 7 \& 8, Addison-Wesley, Reading, MA (1962). 
Smythe, W.R., Static and Dynamical Electricity, $3^{\text {rd }}$ edition, chap. 7, McGrawHill, New York (1969).

1-2 For example: Wilson, M.N., Superconducting Magnets, Clarendon Press, Oxford (1983).

1-3 Magnet calculations are presented in many volumes of the AIP Proceedings of The US Particle Accelerator Schools (http://uspas.fnal.gov); one example is:

Willen, E., P. Dahl, and J. Herrera, Superconducting Magnets, AIP Conference Proceedings 153, American Institute of Physics, New York (1987).

1-4 For example: American Magnetics, Inc., 112 Flint Rd., Oakridge, TN 37831 , www.americanmagnetics.com.

1-5 Lee, E., Solenoidal Transport for Heavy Ion Fusion, Nuclear Instruments and Methods in Physics Research, Volume 544, Issues 1-2 (2005).

1-6 Barnard, J.J., et al., Accelerator and Ion Beam Tradeoffs for Studies of Warm Dense Matter, Proceedings of the 2005 Particle Accelerator Conference, Knoxville TN (05/16-20/2005).

1-7 Tosca $^{\odot}$ is commercially available. Contact: Vector Fields Inc., 1700 N. Farnsworth Avenue, Aurora, IL 60505, USA, http://www.vectorfields.com.

Another code widely used for magnet computations is Poisson Superfish, which is available from the Los Alamos Accelerator Code Group:

Halback, K. and R.F. Holsinger, SUPERFISH-A Computer Program for Evaluation of RF Cavities with Cylindrical Symmetry, Particle Accelerators 7, (1976), and "Poisson Superfish Documentation" by James H. Billen and Lloyd M. Young, Los Alamos National Laboratory Report \# LA-UR-96-1834. The software can be downloaded at http://laacg1.lanl.gov (despite the title, magnet calculations are included).

\section{Acknowledgements}

The authors thank Ms. Lynn Heimbucher for her great effort and patience over several years while shepherding this report through its many revisions. Helpful discussions were held with Shlomo Caspi, Andy Faltens, Alex Friedman, Enrique Henestroza, Steven Lund, and William Waldron.

This work was supported by the Director, Office of Science, and Office of Fusion Energy Sciences, of the U.S. Department of Energy under Contract No. DE-AC02-05CH11231. 


\section{Solenoid Basics}

\subsection{Model assumptions and equations}

We wish to calculate the magnetic field $(\vec{B})$ of a system of solenoids that is suitable for the transport and focusing of ion beams. Our initial model assumes the field is constant in time and has strict azimuthal symmetry around the system axis, which is the zaxis of a cylindrical coordinate system $(r, \theta, z)$. Since the variable $r$ denotes perpendicular distance from the $z$ axis, the distance from the coordinate origin to field point $\vec{r}$ is $|\vec{r}|=\sqrt{r^{2}+z^{2}}$, and its Cartesian coordinates are $[x=r \cos (\theta), y=r \sin (\theta), z]$. The wire current density in the model has only the single component $J_{\theta}(r, z)$, which is the source for the two magnetic field components $\left[B_{r}(r, z), B_{z}(r, z)\right]$. Magnetized materials provide an additional source $J_{\theta}^{\text {mag }}(r, z)$, which is equal to the curl of the magnetization density $\vec{M}$, with components $\left[M_{r}(r, z), M_{z}(r, z)\right]$. In this case $J_{\theta}+J_{\theta}^{\mathrm{mag}}$ is the total source for $\vec{B}$. Deviations from axisymmetry are considered only in sections 14, 15, 21, 22 and 23 . The magnetization of induction cores, with idealized current density and field components $\left(J_{r}, J_{z}, B_{\theta}, M_{\theta}\right)$, is not treated here.

Wire leads and windings are assumed to be paired such that field aberrations due to deviations from solenoidal symmetry are cancelled down to a negligible level. This includes cancellation of the small $z$ component of current that is present in a single (helical) coil of wire by returning the current in a second coil on top of it. Also, the smallscale variations of field inside and close to individual wire or cable sections are ignored, i.e. a smoothed model for $J_{\theta}(r, z)$ is adopted. In effect, the dominant collective field from the current density is calculated here, without any distinction between wire and cable, and small-scale variations are left for detailed magnet computations. However, one must be careful when representing actual currents by a smoothed $J_{\theta}(r, z)$. For example the correct dipole moment of a solenoid (see eqns. $2.11-2.13$ ) should be recovered in the smoothed model.

SI units (Tesla, Ampere, meter) are used throughout this report. The relevant field equations in the absence of magnetic materials are then

$$
\vec{\nabla} \times \vec{B}=\mu_{0} \vec{J}, \quad \vec{\nabla} \cdot \vec{B}=0
$$

where $\mu_{0}=4 \pi x 10^{-7} \mathrm{Tm} / A$. Equation (2.1a) requires $\vec{\nabla} \cdot J=0$. The field conventionally denoted by $\vec{H}$ is essentially equivalent to $\vec{B}$ in the absence of material magnetization $\left(\vec{H}=\vec{B} / \mu_{0}-\vec{M}\right)$. It is not used until section 16 , where its role is discussed in detail. 
To convert any equation to Gaussian (cgs) form, substitute $\mu_{0} \rightarrow 4 \pi / c$. Unit conversions are 1.0 Tesla $=10^{4}$ Gauss, 1.0 Ampere $=2.99792 \ldots x 10^{9}$ statampere, and $1.0 \mathrm{~m}=10^{2} \mathrm{~cm}$.

Assuming solenoidal symmetry, eqns. $(2.1 \mathrm{a}, \mathrm{b})$ become

$$
\frac{\partial B_{r}}{\partial \mathrm{z}}-\frac{\partial B_{\mathrm{z}}}{\partial r}=\mu_{0} J_{\theta}, \quad \frac{1}{r} \frac{\partial}{\partial r} r B_{r}+\frac{\partial B_{\mathrm{z}}}{\partial \mathrm{z}}=0 .
$$

From these coupled first-order equations one can readily derive the decoupled secondorder equations:

$$
\begin{aligned}
& \frac{1}{r} \frac{\partial}{\partial r} r \frac{\partial B_{\mathrm{z}}}{\partial r}+\frac{\partial^{2} B_{\mathrm{z}}}{\partial \mathrm{z}^{2}}=-\mu_{0} \frac{1}{r} \frac{\partial}{\partial r} r J_{\theta}, \\
& \frac{\partial}{\partial r} \frac{1}{r} \frac{\partial}{\partial r} r B_{r}+\frac{\partial^{2} B_{r}}{\partial \mathrm{z}^{2}}=\mu_{0} \frac{\partial J_{\theta}}{\partial \mathrm{z}} .
\end{aligned}
$$

The operator acting on $B_{z}$ in eqns. $(2.3 \mathrm{a}, \mathrm{b})$ is the scalar Laplacian in cylindrical coordinates with $\partial / \partial \theta=0$, while the operator acting on $B_{r}$ is not quite of this standard form. Both of these field equations can be solved using the Green function for the Laplacian operator - see section 6 . This may seem unnecessarily complicated since integrations are over all three spatial variables $(r, \theta, z)$ to obtain functions of only the two variables $(r, z)$. However, for the cases of greatest interest, the thin and thick uniform current layers, the integrations can be reduced to the single variable $\theta$, and the analysis is not much more complicated than the textbook case of a single current loop. It may seem simpler to use the Biot-Savart formula (also a Green function solution), which yields $\vec{B}$ from a single integration along the wire path - see section 21 , but this is somewhat misleading because the path may be very long and must be specified by a more complicated formula than is used for the smoothed $J_{\theta}(r, z)$. On the other hand, the BiotSavart formula gives fine-scale detail of the field around the wire that is missed by the Green function approach using a smoothed source, and is therefore useful for detailed design. We also employ Fourier and Bessel series and transformations, several types of magnetic potential, and for really complicated cases a finite element code.

If Cartesian components and coordinates are used then eqns. $(2.3 \mathrm{a}, \mathrm{b})$ are replaced by the compact vector form

$$
\nabla^{2} \vec{B}=-\mu_{0} \vec{\nabla} x \vec{J}
$$

which can be immediately integrated using the Green function for the Laplacian operator. It is then necessary to project $B_{r}$ from $\vec{B}$; this is only a minor complication, and is equivalent to the solution of eqn. (2.3b) given in section 6 . 


\subsection{Simple field estimate}

Generally we assume $J_{\theta}=0$ at large $|\vec{r}|$. However, to make a rough estimate of field strength we examine an infinite, uniform, thin current layer of $S$ Amperes $/ \mathrm{m}$ :

$$
J_{\theta}(r, z)=S \delta(r-R) \quad \text { all } \mathrm{z}
$$

where $\delta(x)$ is the Dirac delta function. Eqns. $(2.2 \mathrm{a}, \mathrm{b})$ then give

$$
B_{z}=\mu_{0} S \Theta(r-R), \quad B_{r}=0
$$

Here $\Theta(x)$ denotes a downwards unit step at $x=0$, with $d \Theta(x) / d x=-\delta(x)$ :

$$
\Theta(x)= \begin{cases}1, & x<0 \\ 0, & x>0 .\end{cases}
$$

For a bore field of $1.0 \mathrm{~T}$ we need

$$
S=\frac{B_{z}}{\mu_{0}}=\frac{1.0}{4 \pi \times 10^{-7}}=795.8 \times 10^{3} \mathrm{~A} / \mathrm{m}
$$

In round numbers $\mathrm{S}=.80 \mathrm{MA} / \mathrm{m}$ for $1.0 \mathrm{~T}$ (or $800 \mathrm{~A} / \mathrm{cm}$ for $1.0 \mathrm{kG}$ ) for a long magnet, but $\mathrm{S}$ must be somewhat higher for short magnets.

Solenoidal fields for heavy ion beam transport must be very strong, due to the large ion momenta at kinetic energies of interest and near parallelism of field lines and trajectories. However, superconducting wire or cable can carry very high current densities at high fields, so a high field magnet can be made with a relatively thin current layer. Examples of "engineering-averaged critical current density" follow-see section 20.

Critical fields and current density at $4.2 \mathrm{~K}$

\begin{tabular}{|lcc|}
\hline Superconductor & wire field & peak J \\
$\mathrm{NbTi}$ & $6.0 \mathrm{~T}$ & $5 \times 10^{8} \mathrm{~A} / \mathrm{m}^{2}$ \\
$\mathrm{Nb}_{3} \mathrm{Sn}$ & $10.0 \mathrm{~T}$ & $7.5 \times 10^{8} \mathrm{~A} / \mathrm{m}^{2}$ \\
$\mathrm{Nb}_{3} \mathrm{Sn}$ & $15.0 \mathrm{~T}$ & $4.8 \times 10^{8} \mathrm{~A} / \mathrm{m}^{2}$ \\
\hline
\end{tabular}


These current densities take into account space for $\mathrm{Cu}$ stabilizer and insulation, as well as actual superconductor, but there is no additional safety factor or allowance for gaps. They are lower than the critical current densities for the pure superconductor by factors of 3 10. For example, a bore field of $10.0 \mathrm{~T}$ may require $S \approx 10.0 \mathrm{MA} / \mathrm{m}$, which can be made (in principle) using $\mathrm{Nb}_{3} \mathrm{Sn}$ at $4.2 \mathrm{~K}$ with current layer thickness $=\mathrm{S} / \mathrm{J}=10^{7} / 7.5 \times 10^{8}=.0133 \mathrm{~m}$. A safety factor of $30 \%$ might be added.

When the radial distance from the magnet axis out to the wire is greater than a few times the total layer thickness, we can model the current layer as an annulus of infinitesimal thickness for the purpose of computing the field near the magnet axis. However, high field solenoids, especially those using normal conductor, often have total current layer thickness comparable to the vacuum bore radius or greater. This more complicated geometry is analyzed in section 9 .

\subsection{Current distributions, global picture, and moments}

The field of a solenoid is closely related to a radial integral of the current density, which we call the "cumulative current distribution":

$$
S(r, z) \equiv \int_{r}^{\infty} d r^{\prime} J_{\theta}\left(r^{\prime}, z\right),
$$

which has net current distribution $S(z)=S(r=0, z)$. If $\mathrm{S}(\mathrm{r}, \mathrm{z})$ varies slowly with $\mathrm{z}$ then eqns. $(2.2 \mathrm{a}, \mathrm{b})$ appear to have the approximate solution

$$
\begin{aligned}
& B_{z} \approx \mu_{0} S(r, z), \\
& B_{r} \approx-\frac{\mu_{0}}{r} \int_{0}^{r} d r^{\prime} r^{\prime} \frac{\partial S\left(r^{\prime}, z\right)}{\partial z} .
\end{aligned}
$$

However, most solenoids have abrupt current layer ends, so eqns. (2.8a,b) give only a very rough approximation inside the magnet bore and wire. They are incorrect at large $r$. Sections 3-12 are devoted to obtaining accurate formulas for all ranges of $(\mathrm{r}, \mathrm{z})$.

To avoid confusion we mention here that the symbol $S$, which is always a radial integration of $J_{\theta}$, appears in four closely related roles: the simple scale value $S$, the cumulative current distribution $S(r, z)$, the net current distribution

$S(z)$, and the cumulative current distribution $S(r)$ that is present when $S(r, z)$ is uniform in $z$ between specified magnet ends. If $J_{\theta}$ is uniform in $r$ between radii $R_{1}$ and $R_{2}$ and vanishes otherwise, then $S(r, z)=S(z)$ for $0 \leq r \leq R_{1}$, and decreases linearly to zero between $R_{1}$ and $R_{2}$. Solenoids with uniform current density produce fields suitable for beam transport. However, there are applications that require a more complicated distribution within a single magnet, for example to make a very uniform bore field. Such fields can be designed by superposing the fields of several simple layers. 
The symbol $I$ denotes current in a wire, current in a ring, or total azimuthal wire current in a magnet, depending on context. In the latter two cases $I=\int d z S(z)$. The value of the line integral $\int d \vec{s} \bullet \vec{B} / \mu_{0}$ along a closed path in vacuum equals the net enclosed wire current $I$. This result is still correct when magnetized materials are enclosed by the integration path, as will be shown at the end of section 16 .

Since $\vec{\nabla} \cdot \vec{B}=0$ and $\vec{B}$ vanishes at large $|\vec{r}|$ (at least as fast as $|\vec{r}|^{-3}$ ), there must be a "return flux". The total flux through any plane with fixed coordinate $\mathrm{z}$ is

$$
\int_{0}^{\infty} d r 2 \pi r B_{z}(r, z)=0
$$

and (ideally) all flux lines form closed loops at fixed values of $\theta$. However, for a very long system the far field (i.e. the field at large $|\vec{r}|$ much greater than the system length) gets mixed up with the Earth's field or the fields from other sources, so the concept of return flux is actually slightly ambiguous. The external field produced outside of the current layer but close to the magnet system, usually with the sign of $B_{z}$ reversed from the adjacent bore field, is of great interest - see the figure below. For example, it may interfere with the functioning of induction cores in a linac or with nearby instrumentation. The external field is calculated in several sections - especially section 11 .

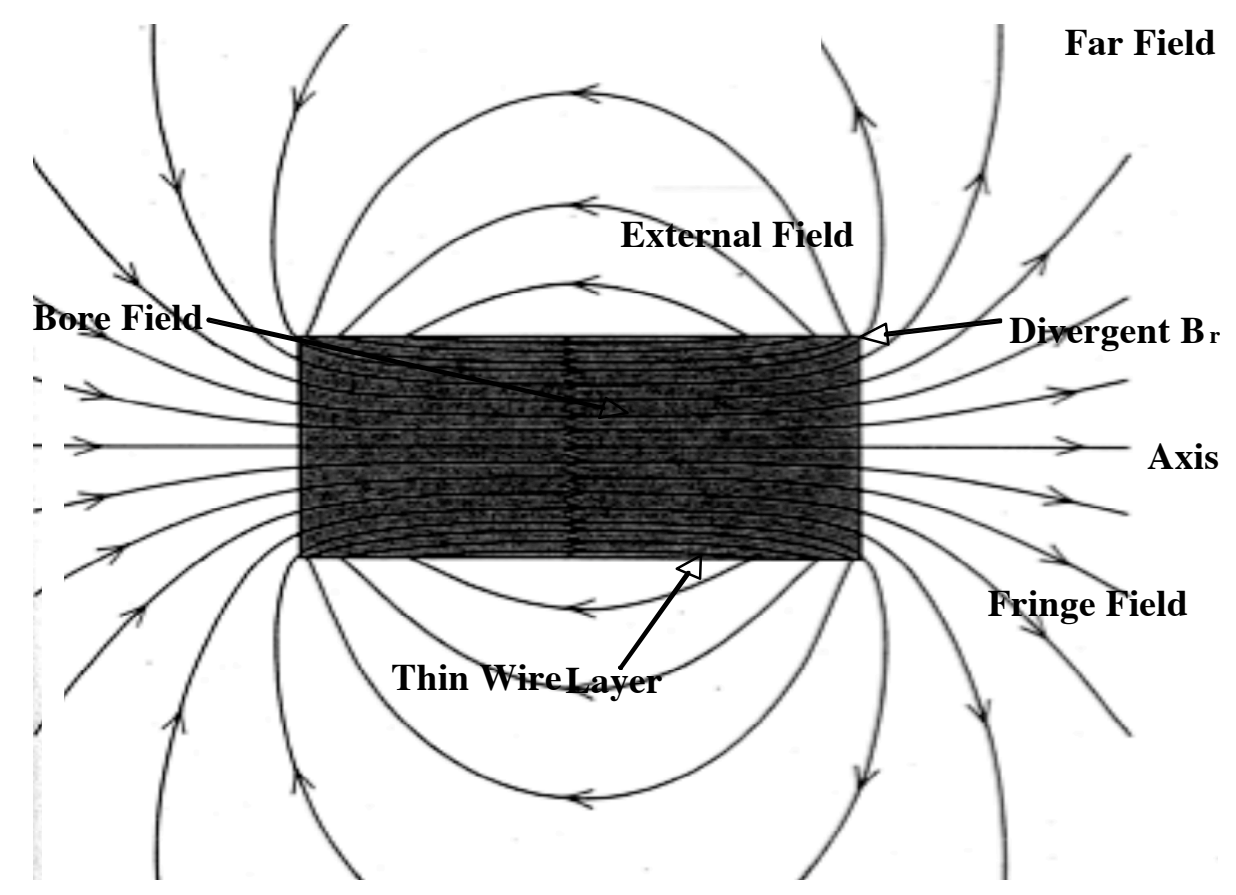

One (rather expensive) way to reduce or eliminate the far field is to place an additional current layer with reverse polarity at larger radius than the primary layer. For 
example a very long, thin current layer $S$ at radius $R$ can be surrounded by a second layer $-S / 4$ at $2 R$, resulting in zero net flux in the region $r<2 R$. This causes a reduction of the bore field from $1.0 \mu_{0} S$ to $.75 \mu_{0} S$. A permeable annular yoke can also be used to confine the return flux and reduce the external field, without any reduction of the bore field - see section 18 .

For visualizing and calculating the field outside the magnet bore it is useful to define "direct" and "residual" fields:

$$
\begin{aligned}
& \vec{B}^{\text {direct }} \equiv \mu_{0} S(r, z) \hat{e}_{z} \\
& \vec{B}^{\text {residual }} \equiv \vec{B}-\vec{B}^{\text {direct }}
\end{aligned}
$$

A "direct flux" is defined as

$$
F(z)=\int_{0}^{\infty} d r 2 \pi r \mu_{0} S(r, z)=\int_{0}^{\infty} d r \pi r^{2} \mu_{0} J_{\theta}(r, z)
$$

where we integrated by parts to obtain the last expression.

A single loop of wire of radius $R$ and carrying azimuthal current $I$ has magnetic dipole moment $\pi R^{2} I$. Magnetic dipole moments add for a system with solenoidal symmetry, so in general we have for a system's total magnetic dipole moment $m_{1}$ :

$$
\begin{aligned}
& m_{1}=\int_{-\infty}^{+\infty} d z \int_{0}^{\infty} d r \pi r^{2} J_{\theta}=\int_{-\infty}^{+\infty} d z \frac{F(z)}{\mu_{0}} \\
& =2 \pi \int_{-\infty}^{+\infty} d z \int_{0}^{\infty} d r r S(r, z)
\end{aligned}
$$

Assuming $m_{1}$ is non-zero, the field at very large $|\vec{r}|$, i.e. the far field-see section 11 , is

$$
B_{r}^{\mathrm{far}}=\frac{\mu_{0} m_{1}}{4 \pi} \frac{3 r z}{{\sqrt{r^{2}+z^{2}}}^{5}}, \quad B_{z}^{\mathrm{far}}=\frac{\mu_{0} m_{1}}{4 \pi} \frac{2 z^{2}-r^{2}}{{\sqrt{r^{2}+z^{2}}}^{5}} .
$$

Higher order moments, which generate field components that fall off more rapidly with $|\vec{r}|$, are also present. However, the coordinate origin can usually be positioned such that the second moment $\left(m_{2}=\int d z \int d r^{2 \pi} r^{2} z J_{\theta}\right)$ vanishes.

Solenoidal multipoles and their moments should not be confused with transverse multipole magnets that are commonly used for beam transport and focusing, and which are referred to as dipoles, quadrupoles, sextupoles, etc. 
So far we have define two radial moments weighted by $J_{\theta}$ :

$$
\begin{aligned}
& S(z)=\int_{0}^{\infty} d r J_{\theta}(r, z), \\
& S(z) \overline{r^{2}}(z)=\int_{0}^{\infty} d r r^{2} J_{\theta}(r, z)=2 \int_{0}^{\infty} d r r S(r, z) .
\end{aligned}
$$

Additional radial moments weighted by $J_{\theta}$ such as $S \overline{r^{4}}, S \overline{r^{6}}, \ldots$ will be used in section 11 to evaluate the external field. Note that $\overline{r^{2}}, \overline{r^{4}}$, etc. can actually be negative if $J_{\theta}$ varies in sign as a function of $\mathrm{r}$, so they are not true mean values.

As mentioned, care is needed when representing a magnet by a smooth current density. Consider a simple wire coil of $n$ turns at radius $R$ with turn-to-turn longitudinal spacing $\delta$. If the wire current is $I$ then the net azimuthal current is $n I$ and the net magnetic dipole moment is $m_{1}=\pi R^{2} n I$. However, the effective coil length is $\ell=n \delta$, not $(n-1) \delta$, since each loop would be centered in a strip of length $\delta$ for an accurate finite difference calculation. The correct current distribution is $S=n I / \ell=I / \delta$, which is consistent with the value $m_{1}=\ell \pi R^{2} S$ obtained from eqn. (2.12). The physical length of the coil alone, taking into account that it is actually a helix, is also $\ell$ (plus one wire thickness). The longitudinal current of the coil is $I$, which would be the source of an azimuthal field. However, this is assumed to be cancelled by a second coil at slightly larger radius.

\subsection{Vector potential and flux lines}

It is sometimes helpful to work with one of four different magnetic potentials - see sections 5 and 19 . Here we only mention the vector potential $\vec{A}$, with $\vec{B}=\vec{\nabla} x \vec{A}$, which for a solenoidal system can be reduced to the single component $A_{\theta}(r, z)$ :

$$
B_{r}=-\frac{\partial A_{\theta}}{\partial z}, B_{z}=\frac{1}{r} \frac{\partial}{\partial r} r A_{\theta} .
$$

A flux surface is characterized by

$$
r A_{\theta}(r, z)=\text { constant. }
$$

This is easily shown by considering any flux line that lies on a particular flux surface. The line has constant $\theta$, so it is only necessary to show that $r A_{\theta}$ is constant along it. Since by definition the flux line element $d \vec{r}$ is parallel to $\vec{B}$, the differential of $r A_{\theta}$ along the line is 


$$
d\left(r A_{\theta}\right)=\left(\frac{\partial}{\partial r} r A_{\theta}\right) d r+\left(\frac{\partial r A_{\theta}}{\partial z}\right) d z=r\left(B_{z} d r-B_{r} d z\right)=r \hat{e}_{\theta} \cdot(\vec{B} x \vec{d} r)=0
$$

If adjacent flux surfaces have constants $r A_{\theta}=C_{1}$ and $C_{2}$, then the magnetic flux contained between the two surfaces is $2 \pi\left(C_{2}-C_{1}\right)$. The magnitude of the field strength midway between the two flux surfaces is approximately

$$
B=\frac{C_{2}-C_{1}}{\nabla \cdot r}
$$

where $\Delta$ is the local normal distance between the surfaces and $r$ is the radial distance from the axis to the midpoint between the surfaces. Due to the factor of $r^{-1}$ in eqn. (2.18), the magnitude of $\vec{B}$ is not simply proportional to the inverse spacing between flux surfaces $\left(\Delta^{-1}\right)$ as is sometimes assumed, although this can be a useful visual aid in a region where $r$ is nearly constant. The local density of flux lines through any plane normal to $\vec{B}$ can be specified to be proportional to $\overrightarrow{|B|}$, but this is not usually done when making flux line plots. A flux surface has the topology of a torus, and a system made from more than one magnet may have several flux surfaces corresponding to a given value of $r A_{\theta}$.

Another way to generate a flux line is to solve the equation

$$
\frac{d \vec{r}}{d s}=\frac{\vec{B}(\vec{r})}{|\vec{B}(\vec{r})|}
$$

starting from some point on the line of interest. Here $\vec{r}(s)$ is simply the parameterization of the line, with s denoting distance along the line. For example the starting point could be on the surface of an acceleration gap where unwanted electrons are emitted.

\subsection{Stress and energy}

Material stress is not the topic of this report, but it is a major concern in the design of high field magnets, so a simple estimate is presented here. The force density from the field pushing on the current layer is

$$
\vec{J} x \vec{B}=\hat{e}_{r} J_{\theta} B_{z}-\hat{e}_{z} J_{\theta} B_{r}
$$

Since the wire is in mechanical equilibrium, this force density must equal the divergence of the material stress tensor. A detailed stress analysis is too complicated to summarize here-see e.g. ref [1-2], but it is seen from eqn. (2.20) that the longitudinal magnetic force tends to compress a wire layer while the radial magnetic force pushes it outwards against 
a restraining collar of the magnet assembly. There is no material stress in the vacuum bore, so at the collar there is approximately a material force per $m^{2}$ pushing outwards:

$$
F \approx \int d r J_{\theta} B_{z} \approx \frac{B_{w}^{2}}{2 \mu_{0}},
$$

where $B_{w}$ is the longitudinal field at the inner edge of the wire layer - roughly the peak bore field. Suppose $B_{w}=1.0 T$, then $F \approx\left((1.0)^{2} / 2 \times 4 \pi \times 10^{-7}\right) \approx 4 \times 10^{5} \mathrm{~N} / \mathrm{m}^{2}=4.0 \mathrm{Bar}$, which is quite modest. However a 15.0T bore field produces about 900 Bar, which requires substantial support to prevent wire movement.

The external field of a solenoid without a permeable return yoke extends to a large distance compared to the field of a transverse quadrupole magnet. The estimation of stress and wire field in a system of solenoids must therefore include the field contributions of nearby magnets as well as that of the magnet of interest. This is particularly the case for a transport lattice made of closely spaced solenoids.

Since solenoidal transport systems for energetic ions are expected to have high fields in large-bore magnets, they also have large stored field energy. In the absence of magnetic materials this is

$$
W=\int d^{3} r|\vec{B}|^{2} / 2 \mu_{0}
$$

For example if a $1.0 \mathrm{~m}^{3}$ volume is filled with a $15 \mathrm{~T}$ field, then the stored energy is $15^{2} /\left(2 \times 4 \pi \times 10^{-7}\right) \approx 90 \mathrm{MJ}$. The related topic of magnet cooling is not discussed here.

\section{Near-Axis Field}

For simulating beam dynamics it is often sufficient to accurately approximate the magnetic field near the system axis, e.g. out to $50 \%$ of the radius of the nearest wire layer. A general treatment is presented here and some useful examples of the on-axis field are given in section 4 .

\subsection{On-axis field and near-axis expansion}

Recall eqn. (2.3a) for $B_{z}$ :

$$
\nabla^{2} B_{z}=-\mu_{0} \frac{1}{r} \frac{\partial}{\partial r} r J_{\theta}
$$


This may be formally integrated using the Green function for the 3-d scalar Laplacian operator - see section 6 :

$$
B_{z}=\frac{\mu_{0}}{4 \pi} \int d^{3} r^{\prime} \frac{\frac{1}{r^{\prime}} \frac{\partial}{\partial r^{\prime}} r^{\prime} J_{\theta}^{\prime}}{\left|\vec{r}^{\prime}-\vec{r}\right|}
$$

or explicitly in cylindrical coordinates,

$$
B_{z}=\frac{\mu_{0}}{4 \pi} \int_{-\infty}^{+\infty} d z^{\prime} \int_{0}^{\infty} d r^{\prime} r^{\prime} \int_{0}^{2 \pi} d \theta^{\prime} \frac{\frac{1}{r^{\prime}} \frac{\partial}{\partial r^{\prime}} r^{\prime} J_{\theta}\left(r^{\prime}, z^{\prime}\right)}{\sqrt{\left(z^{\prime}-z\right)^{2}+r^{\prime 2}+r^{2}-2 r^{\prime} r \cos \left(\theta^{\prime}-\theta\right)}}
$$

Here and in similar integrations we may set $\theta=0$ inside the integrand since by symmetry $B_{z}$ is a function only of $\mathrm{r}$ and $\mathrm{z}$. After an integration by parts in $r^{\prime}$ we get

$$
B_{z}(r, z)=\frac{\mu_{0}}{4 \pi} \int_{-\infty}^{+\infty} d z^{\prime} \int_{0}^{\infty} d r^{\prime} r^{\prime} J_{\theta}\left(r^{\prime}, z^{\prime}\right) \int_{0}^{2 \pi} d \theta^{\prime} \frac{\left(r^{\prime}-r \cos \left(\theta^{\prime}\right)\right)}{{\sqrt{\left(z^{\prime}-z\right)^{2}+r^{\prime 2}+r^{2}-2 r^{\prime} r \cos \left(\theta^{\prime}\right)}}^{3}}
$$

This formula is completely general for a static solenoidal field, and it gives the right answer even when the current layer extends to infinity in $z$.

To get the near-axis field, we first evaluate the on-axis field:

$$
B_{0}(z) \equiv B_{z}(r=0, z)=\frac{\mu_{0}}{2} \int_{-\infty}^{+\infty} d z^{\prime} \int_{0}^{\infty} d r^{\prime} \frac{r^{\prime 2} J_{\theta}\left(r^{\prime}, z^{\prime}\right)}{{\sqrt{\left(z^{\prime}-z\right)^{2}+r^{\prime 2}}}^{3}}
$$

If $B_{0}(z)$ is accurately determined from this expression or in some other way, such as measurements on a real magnet system, then $B_{0}(z)$ may be used to generate a power series solution of eqn. (3.1) that is valid for small $r$ (where $J_{\theta}=0$ ). From

$$
\frac{1}{r} \frac{\partial}{\partial r} r \frac{\partial B_{z}}{\partial r}=-\frac{\partial^{2} B_{z}}{\partial z^{2}}
$$

we get by iteration:

$$
B_{z}(r, z)=B_{0}-\frac{B_{0}^{\prime \prime} r^{2}}{4}+\frac{B_{0}^{i v} r^{4}}{64}-\frac{B_{0}{ }^{v i} r^{6}}{2304}+\ldots
$$

The radial field component is easily obtained from $\vec{\nabla} \cdot \vec{B}=0$ by integrating in r: 


$$
B_{r}(r, z)=-\frac{1}{r} \int_{0}^{r} d r^{\prime} r^{\prime} \frac{\partial B_{z}\left(r^{\prime}, z\right)}{\partial z}=-\frac{B_{0}^{\prime} r}{2}+\frac{B_{0}^{\prime \prime \prime} r^{3}}{16}-\frac{B_{0}^{v} r^{5}}{384}+\frac{B_{0}^{v i i} r^{7}}{18432}-\ldots \ldots
$$

The leading term in each of the above expansions of $B_{z}$ and $B_{r}$ is used when computing the linearized dynamics of beam ions. Higher order (non-linear in $r$ ) terms are referred to as "fringe field aberrations" in this context. Two terms from each of the expansions usually give an accurate field representation inside the vacuum bore, especially near a magnet center, but the expansion for $B_{z}$ fails in the wire and beyond, where the field component generally reverses sign. The expansion for $B_{r}$ may give a fair approximation in the wire except near magnet ends. When truncating the expansions after some power of r, $B_{r}$ should be represented by one power of $r$ higher than $B_{z}$ in order to preserve $\vec{\nabla} \cdot \vec{B}=0$. Crossing a thin current layer of net current distribution $S(z), B_{z}$ jumps by $-\mu_{0} S(z)$ but $B_{r}$ is continuous. However, $B_{r}$ has logarithmic singularities at the ends of the layer - see section 8. For large $|z|$, well beyond any magnet end, the power series expansions are valid out to values of $r$ comparable to the longitudinal distance from the magnet end. But the expansions do not correctly yield the reversal of $B_{z}$ at larger values of $r$. Also, because of the high order derivatives, the expansions of $B_{r}$ and $B_{z}$ may be unreliable using measured $B_{0}$.

To check the accuracy of the near-axis expansion $(3.6 a, b)$ we examine the thin semi-infinite current layer,

$$
J_{\theta}=S \Theta(z) \delta(r-R)
$$

We get from eqn. (3.5) - see section 4:

$$
B_{0}(z)=\frac{\mu_{0} S}{2}\left(1-\frac{z}{\sqrt{z^{2}+R^{2}}}\right)
$$

In this simple case fringe field aberrations are maximum at $\mathrm{z}=0$ for $B_{r}$ and at $z \approx \pm .5 R$ for $B_{z}$. Approximating the fields by only two terms works well for $\mathrm{r}<.5 \mathrm{R}$, but for $\mathrm{r}=.8 \mathrm{R}$ (the largest value one would consider for beam transport) the convergence is slow, as shown in the following tables. Note that the approximate $B_{z}$ oscillates as higher order terms are added. 


\begin{tabular}{|ccc|}
\hline Number of expansion terms & $B_{z} / \mu_{0} S_{\text {at r }=.8 \mathrm{R}, \mathrm{z}=.5 \mathrm{R}}$ & $B_{r} / \mu_{0} S$ at $\mathrm{r}=.8 \mathrm{R}, \mathrm{z}=0$ \\
1 & .276393 & .200000 \\
2 & .207701 & .248000 \\
3 & .190116 & .267200 \\
4 & .189066 & .276160 \\
5 & .190881 & .280676 \\
exact & .191960 & .286062 \\
\end{tabular}

\begin{tabular}{|ccc|}
\hline Number of expansion terms & $B_{z} / \mu_{0} S$ at $\mathrm{r}=.5 \mathrm{R}, \mathrm{z}=.5 \mathrm{R}$ & $B_{r} / \mu_{0} S$ at $\mathrm{r}=.5 \mathrm{R}, \mathrm{z}=0$ \\
1 & .276393 & .125000 \\
2 & .249560 & .136719 \\
3 & .246877 & .138550 \\
4 & .246814 & .138884 \\
5 & .246857 & .138949 \\
exact & .246867 & .138967 \\
\hline
\end{tabular}

\subsection{Useful integrals}

The following (non-trivial but easily verified) indefinite integrals are of use in subsequent sections:

$$
\begin{aligned}
& \int \frac{d x}{{\sqrt{x^{2}+a^{2}}}^{3}}=\frac{x}{a^{2} \sqrt{x^{2}+a^{2}}} \\
& \int \frac{d x}{\sqrt{x^{2}+a^{2}}}=\log \left(\frac{x+\sqrt{x^{2}+a^{2}}}{a}\right)=\sinh ^{-1}(x / a) \\
& \int \frac{d x}{\sqrt{a^{2}-x^{2}}}=\sin ^{-1}\left(\frac{x}{a}\right) \\
& \int \frac{d x}{a^{2}-x^{2}}=\frac{1}{a} \tanh ^{-1}\left(\frac{x}{a}\right) \\
& \int \frac{d x}{a^{2}+x^{2}}=\frac{1}{a} \tan ^{-1}\left(\frac{x}{a}\right) \\
& \int \frac{d x}{\sqrt{a^{2}+x^{2}}}=\frac{x}{3 a^{4}}\left(\frac{2}{\sqrt{a^{2}+x^{2}}}+\frac{a^{2}}{{\sqrt{a^{2}+x^{2}}}^{3}}\right)
\end{aligned}
$$




$$
\int \frac{d x}{\left(a x^{2}+b\right) \sqrt{f x^{2}+g}}=\frac{1}{\sqrt{b(a g-b f)}} \tan ^{-1}\left(\frac{x \sqrt{a g-b f}}{\sqrt{b\left(f x^{2}+g\right)}}\right)
$$

[for $a, b, f, g>0$ and $a g-b f>0]$.

The surprising integral $(3.10 \mathrm{~g})$ is used in section 9 to evaluate $B_{z}(r, z)$ for a uniform, thick current layer.

\section{Design Formulas for the On-Axis Field}

To calculate the on-axis field, denoted $B_{0}(z)$, we may replace a moderately thick, uniform current layer with a thin layer at its average radius $(\mathrm{R})$ with surprisingly little error - see section 9. Making this approximation leads to many useful formulas. The replacement current density has the simple form:

$$
J_{\theta}(r, z)=S(z) \delta(r-R)
$$

and eqn. (3.5) becomes

$$
B_{0}(z)=\frac{\mu_{0}}{2} \int_{-\infty}^{+\infty} d z^{\prime} \frac{R^{2} S\left(z^{\prime}\right)}{{\sqrt{\left(z^{\prime}-z\right)^{2}+R^{2}}}^{3}}
$$

\subsection{Simple cases}

$\underline{\text { Case } 1}$ Constant $S(z)=S$ for all z:

Using the integral (3.10a) we recover eqn. (2.5a),

$$
B_{0}=\left.\frac{\mu_{0} S}{2} \frac{\left(z^{\prime}-z\right)}{\sqrt{\left(z^{\prime}-z\right)^{2}+R^{2}}}\right|_{-\infty} ^{+\infty}=\mu_{0} S
$$

$\underline{\text { Case } 2}$ Semi-infinite Layer:

$$
S(z)=S \Theta\left(z-z_{0}\right)= \begin{cases}S & -\infty<z<z_{0} \\ 0 & z_{0}<z<\infty\end{cases}
$$

A very useful formula is derived by changing the upper limit of integration over $z^{\prime}$ to $z_{0}$ in eqn. (4.3): 


$$
B_{0}(z)=\left.\frac{\mu_{0} S}{2} \frac{\left(z^{\prime}-z\right)}{\sqrt{\left(z^{\prime}-z\right)^{2}+R^{2}}}\right|_{-\infty} ^{z_{0}}=\frac{\mu_{0} S}{2}\left[1-\frac{\left(z-z_{0}\right)}{\sqrt{\left(z-z_{0}\right)^{2}+R^{2}}}\right] .
$$

Note the symmetry around $z_{0}$, with $B_{0}\left(z_{0}\right)=\mu_{0} S / 2$. We can build up many other cases of interest by superposition using this result.

Case 3 Lens with current layer length $\ell$, centered at $z=0$ :

We apply eqn. (4.5) with positive and negative current layers ending respectively at $\ell / 2$ and $-\ell / 2$ :

$$
B_{0}^{\text {lens }}(z)=\frac{\mu_{0} S}{2}\left[\frac{(z+\ell / 2)}{\sqrt{(z+\ell / 2)^{2}+R^{2}}}-\frac{(z-\ell / 2)}{\sqrt{(z-\ell / 2)^{2}+R^{2}}}\right] .
$$

This formula is equivalent to the well-known textbook result [4-1]:

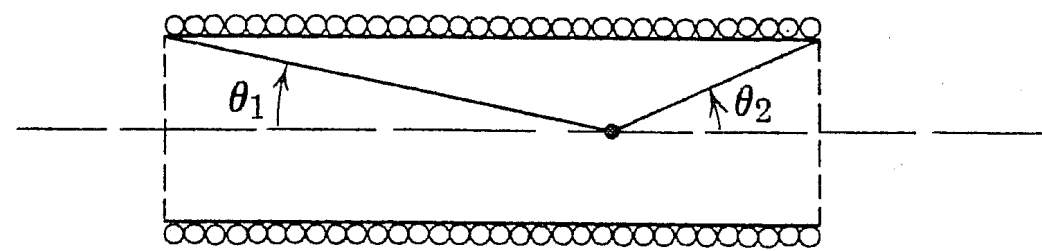

$$
B_{0}=\frac{\mu_{0} N I}{2}\left[\cos \left(\theta_{1}\right)+\cos \left(\theta_{2}\right)\right],
$$

where $\mathrm{N}$ is the number of wire loops per meter, each carrying current $\mathrm{I}$, and the angles are to points at the wire layer ends drawn from the point of interest on the $\mathrm{z}$ axis (see figure). The field at the lens midpoint $(r=0, z=0)$ is

$$
B_{0}^{\text {lens }}(0)=\frac{\mu_{0} S}{\sqrt{1+4 R^{2} / \ell^{2}}},
$$

so a lens of length $\ell=2 R$ has peak field on axis reduced to $\mu_{0} S / \sqrt{2}=.7071 \mu_{0} S$. For $|z|$ large, the leading order expansion term of eqn. (4.6) is

$$
B_{0}^{\text {lens }} \rightarrow \frac{\mu_{0} S R^{2} \ell}{2|z|^{3}},
$$


consistent with eqn. (2.13b), with magnetic dipole moment $m_{1}=\pi R^{2} \ell S$. The on-axis field of a short lens $\left(\mu_{0} S=1.0 \mathrm{~T}, \mathrm{R}=.25 \mathrm{~m}, \ell=1.0 \mathrm{~m}\right)$ is displayed below.

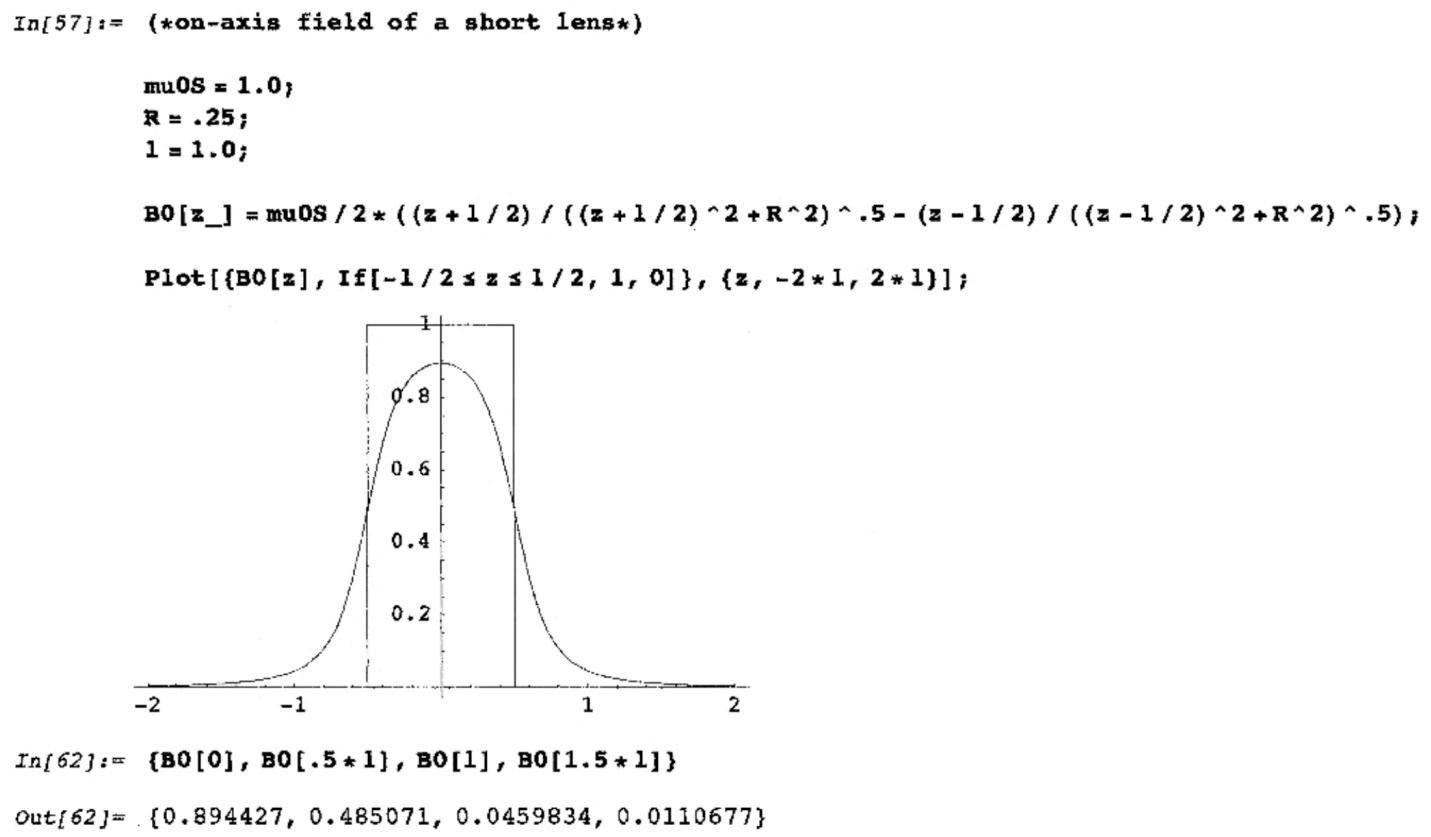

$\left.I_{n}[62\}:=\quad \mathrm{BO}[0], \mathrm{BO}[.5 * 1], \mathrm{BO}[1], \mathrm{BO}[1.5 * 1]\right\}$

Out $[62]=\{0.894427,0.485071,0.0459834,0.0110677\}$

Case 4 Periodic system of lenses (same polarity) of current layer length $\ell$, gap length $\mathrm{g}$, and period $\mathrm{P}=\ell+\mathrm{g}$ :

$$
B_{0}(z)=\sum_{n=-\infty}^{\infty} B_{0}^{\text {lens }}(z-n P)
$$

Case 5 Gap of length $\mathrm{g}$, centered at $\mathrm{z}=0$, between long solenoids:

We subtract a lens of length $\mathrm{g}$ from an infinitely long solenoid:

$$
B_{0}^{\text {gap }}(z)=\mu_{0} S-B_{0}^{\text {lens }}(z)
$$

At the center of the gap

$$
B_{0}^{\mathrm{gap}}(0)=\mu_{0} S\left(1-\frac{1}{\sqrt{1+4 R^{2} / g^{2}}}\right)
$$


For $\mathrm{g}=2 \mathrm{R}$, the field at the gap center is reduced to $(1-1 / \sqrt{2}) \mu_{0} S=.2929 \mu_{0} S$. To prevent large variations of $B_{0}$ with $\mathrm{z}$ in a periodic system, the gaps must be very short compared with $2 \mathrm{R}$. However, this is not generally necessary for the design of a good periodic transport system if the ion betatron wavelength is longer than $2 \mathrm{P}$. If the polarities are opposed then an antisymmetric "cusp" field is produced around $z=0$.

Case 6 Current ring of radius $R$ :

$$
J_{\theta}=I \delta(r-R) \delta(z)
$$

From eqn. (3.5) or eqn. (4.2) we get

$$
B_{0}^{\text {ring }}(z)=\frac{\mu_{0} I R^{2}}{2{\sqrt{z^{2}+R^{2}}}^{3}}
$$

\subsection{Thick current layer}

The on-axis field of a uniform, thick current layer can be computed from any of the thin layer cases with a simple average over the wire layer radius $R$ :

$$
B_{0}^{\text {thick }}(z)=\int_{R_{1}}^{R_{2}} \frac{d R}{R_{2}-R_{1}} B_{0}^{\text {thin }}(z ; R) \text {, }
$$

where $R_{1}$ and $R_{2}$ are the inner and outer radii of the thick layer. For example eqn. (4.6) for a thin layer lens of length $\ell$ gives, using eqn. (3.10b):

$$
\begin{aligned}
& B_{0}^{\text {thick }}(z)=\int_{R_{1}}^{R_{2}} \frac{d R}{R_{2}-R_{1}} \frac{\mu_{0} S}{2}\left\{\frac{(z+\ell / 2)}{\sqrt{(z+\ell / 2)^{2}+R^{2}}}-\frac{(z-\ell / 2)}{\sqrt{(z-\ell / 2)^{2}+R^{2}}}\right\} \\
& =\frac{\mu_{0} S}{2\left(R_{2}-R_{1}\right)}\left\{(z+\ell / 2) \log \left[\frac{\mathrm{R}_{2}+\sqrt{(z+\ell / 2)^{2}+R_{2}^{2}}}{\mathrm{R}_{1}+\sqrt{(z-\ell / 2)^{2}+R_{1}^{2}}}\right]\right. \\
& \left.-(z-\ell / 2) \log \left[\frac{R_{2}+\sqrt{(z-\ell / 2)^{2}+R_{2}^{2}}}{R_{1}+\sqrt{(z-\ell / 2)^{2}+R_{1}^{2}}}\right]\right\} \text {. }
\end{aligned}
$$

\subsection{Uniform fields}

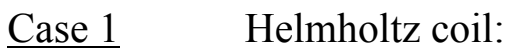


Two rings, each of radius $R$, current $I$, and separated by distance $L$, have on-axis field from eqn. (4.14):

$$
B_{0}(z)=\frac{\mu_{0} I R^{2}}{2}\left[\frac{1}{{\sqrt{(z-L / 2)^{2}+R^{2}}}^{3}}+\frac{1}{{\sqrt{(z+L / 2)^{2}+R^{2}}}^{3}}\right] .
$$

If $L=R$, then $B_{0}^{\prime \prime}(0)=0$ and $B_{0}(0)=(.8) .7155 \mu_{0} I / R$. This is a simple way to make a uniform field close to the coil center, but it is not useful for producing high fields since the field strength in the wire is much larger than at the coil center.

$\underline{\text { Case } 2}$ Nearly uniform field in a long solenoid:

A large volume of strong uniform field can be made by superposing simple solenoids of various lengths, radii and current layer distributions. A mathematically simple version consists of a pair of thin-layer lenses [see eqn. (4.6)] placed symmetrically around $z=0$ at the same radius $R$, but with unequal lengths $\left(\ell_{1}, \ell_{2}\right)$ and distributions $\left(S_{1}, S_{2}\right)$. The derivatives $B_{0}{ }^{\prime \prime}(0)$ and $B_{0}^{i v}(0)$ both vanish if we set

$$
\begin{aligned}
& \frac{\ell_{2}}{2 R}=\sqrt{\frac{3\left(\ell_{1} / 2 R\right)^{2}+10}{4\left(\ell_{1} / 2 R\right)^{2}-3}}, \\
& S_{2}=-S_{1}\left(\frac{\ell_{1}}{\ell_{2}}\right){\sqrt{\frac{1+\left(\ell_{2} / 2 R\right)^{2}}{1+\left(\ell_{1} / 2 R\right)^{2}}}}^{5} .
\end{aligned}
$$

Layer \#2 is shorter than \#1 if $\ell_{1} / 2 R>\sqrt{2.5}$, and has a reversed current. Both layers must have $\ell / 2 R>\sqrt{.75}$. The combined layers produce a somewhat lower field strength than that of layer \#1 alone:

$$
B_{0}(0)=\frac{\mu_{0} S_{1} \ell_{1} / 2}{\sqrt{\left(\ell_{1} / 2\right)^{2}+R^{2}}}+\frac{\mu_{0} S_{2} \ell_{2} / 2}{\sqrt{\left(\ell_{2} / 2\right)^{2}+R^{2}}}
$$

For example, taking $\mu_{0} S_{1}=10.0 \mathrm{~T}$ and $\ell_{1}=4.0 R$, we get $\mu_{0} S_{2}=-3.27097 \mathrm{~T}$, and $\ell_{2}=2.60177 R$, producing $B_{0}(0)=6.35096 T$. The \#1 solenoid alone would produce $B_{0}(0)=8.94427 \mathrm{~T}$. The on-axis field for the double layer at representative points is

\begin{tabular}{|c|c|c|c|c|c|c|}
\hline$z / R$ & 0 & \pm .2 & \pm .4 & \pm .6 & \pm .8 & \pm 1.0 \\
\hline$B_{0}$ & 6.35096 & 6.35096 & 6.35083 & 6.34929 & 6.34063 & 6.30777 \\
\hline
\end{tabular}


There is essentially no variation over $25 \%$ of the $4.0 \mathrm{~m}$ bore length in this example and only .7\% variation over $50 \%$ of the bore length. Transverse variation is similarly small in these zones.

\section{Case $3 \quad$ Uniform field inside an ellipsoidal shell:}

Although this is not particularly useful for beam transport, it is worth mentioning that an ellipsoidal shell of constant current per meter produces an exactly constant field inside the enclosed volume. Denoting the cumulative current layer density

$$
S(r, z)=S \cdot \Theta\left(\frac{r^{2}}{a^{2}}+\frac{z^{2}}{b^{2}}-1\right),
$$

where $\mathrm{a}$ and $\mathrm{b}$ are ellipsoid radii, then

$$
J_{\theta}=-\frac{\partial S(r, z)}{\partial r}=\frac{2 r}{a^{2}} S \cdot \delta\left(1-\frac{r^{2}}{a^{2}}-\frac{z^{2}}{b^{2}}\right)
$$

This current pattern can be created with closely spaced wire loops, each carrying current $I=S \Delta$, where $\Delta$ is the constant longitudinal separation between loops.

Eqn. (3.5) yields

$$
B_{0}(z)=\frac{\mu_{0} S a^{2}}{2} \int_{-b}^{b} d z^{\prime} \frac{\left(1-z^{\prime} 2 / b^{2}\right)}{\sqrt{\left(z^{\prime}-z\right)^{2}+a^{2}\left(1-z^{\prime} 2 / b^{2}\right)}},
$$

from which it may be numerically verified that $B_{0}(z)$ is actually constant for $|z|<b$. $B_{z}$ is therefore also independent of $r$ inside the ellipsoid, and we may write

$$
\begin{aligned}
& B_{z}^{\text {inside }}=\mu_{0} S f(a / b) \\
& f(a / b)=\frac{a^{2}}{b^{2}} \int_{0}^{1} \frac{d y\left(1-y^{2}\right)}{{\sqrt{y^{2}+\frac{a^{2}}{b^{2}}\left(1-y^{2}\right)}}^{3}} .
\end{aligned}
$$

\begin{tabular}{|c|c|c|c|c|c|c|c|}
\hline$a / b$ & 0 & .25 & .50 & 1.0 & 2.0 & 4.0 & $\infty$ \\
\hline$f$ & 1.00000 & .924593 & .826436 & .666667 & .472800 & .296359 & .000000 \\
\hline
\end{tabular}


A proof that $B_{z}$ is constant is mathematically related to the solution for the gravitational potential of an ellipsoid of constant density, obtained in the Eighteenth Century [4-2]. It is found by elementary integration that for $a / b \equiv x<1$,

$$
f(x)=\frac{1}{1-x^{2}}\left[1-\frac{x^{2}}{\sqrt{1-x^{2}}} \log \left(\frac{1+\sqrt{1-x^{2}}}{x}\right)\right],
$$

and for $a / b=x>1$,

$$
f(x)=\frac{1}{x^{2}-1}\left[\frac{x^{2}}{\sqrt{x^{2}-1}} \sin ^{-1}\left(\frac{\sqrt{\mathrm{x}^{2}-1}}{\mathrm{x}}\right)-1\right] .
$$

\section{References}

4-1 See e.g. Reference [1-1], J.D. Jackson, pg. 225.

4-2 Chandrasekhar, S., Ellipsoidal Figures of Equilibrium, chap. 3, Dover Publications, NY (1969).

\section{Vector and Scalar Potentials}

The vector potential $A_{\theta}$ and three scalar potentials, denoted here by $\phi, \varphi$, and $\Phi$, are used in calculating and visualizing solenoidal fields. Each potential has features that make it useful for some applications and a poor choice for others. The scalar potentials $\phi$ and $\varphi$ are described below, while $\Phi$ is a special potential used in Tosca ${ }^{\odot}$ and described in section 19.

\subsection{Vector potential}

As discussed in section 2, the vector potential (only $A_{\theta}$ is needed) is useful for defining and plotting flux surfaces. Another property of $A_{\theta}$ is that the derived field components

$$
B_{r}=-\frac{\partial A_{\theta}}{\partial z}, \quad B_{z}=\frac{1}{r} \frac{\partial}{\partial r} r A_{\theta}
$$

automatically satisfy $\vec{\nabla} \cdot \vec{B}=0$. Also, azimuthal symmetry results in the conservation of canonical angular momentum for ion beam dynamics:

$$
P_{\theta}=r\left(\gamma M v_{\theta}+q \partial A_{\theta}\right)=\text { constant }
$$


for an ion of $\theta-$ momentum $\gamma M v_{\theta}$ and charge state $q$. The constancy of $P_{\theta}$ can be used to eliminate $v_{\theta}$ when $A_{\theta}$ is known.

Unlike the scalar potential $\phi$ described below, the vector potential provides a complete or "global" characterization of the magnetic field that is valid everywhere. Its source is $J_{\theta}$, and if a magnetization current density $J_{\theta}^{m a g}$ is present, it simply adds to the current density of the wire layer. The second order equation for $A_{\theta}$ is

$$
\frac{\partial}{\partial r} \frac{1}{r} \frac{\partial}{\partial r} r A_{\theta}+\frac{\partial^{2} A_{\theta}}{\partial z^{2}}=\frac{\partial B_{z}}{\partial r}-\frac{\partial B_{r}}{\partial z}=-\mu_{0} J_{\theta}
$$

As with eqn. (2.3b) for $B_{r}$, the operator acting on $A_{\theta}$ is not quite the scalar Laplacian, but this does not cause a serious problem in obtaining a Green function solution - see section 6 .

One drawback in using $A_{\theta}$ (and the scalar potentials) to calculate $\vec{B}$ is that it must be differentiated, which is an extra operation that can produce unnecessarily complicated approximate expressions. Also, derivatives generate errors in discretized computations. Another drawback is that $A_{\theta}$ is the azimuthal component of a vector field and must therefore be handled carefully when transforming to a tilted magnet.

\subsection{Magnetic scalar potential}

The field in the bore of a (transverse) magnetic dipole, quadrupole, or higher order beamline element is often characterized by a magnetic scalar potential $\phi$. A magnetic scalar potential can also be used to characterize the bore field in a solenoidal system. In a connected region of vacuum, where $\vec{\nabla} \times \vec{B}=0$, we set

$$
\vec{B}=-\mu_{0} \vec{\nabla} \phi, \quad \nabla^{2} \phi=-\vec{\nabla} \cdot \vec{B} / \mu_{0}=0
$$

with the factor of - $\mu_{0}$ inserted to be consistent with standard convention. This is an attractive, compact representation of structural aberrations, which appear as angular multipoles, e.g. $\phi \sim \phi_{n}(r, z) \cos (n \theta)$, as well as fringe field terms.

By itself $\phi$ cannot be a global solution. It is necessary to connect $\phi$ to its current source through boundary conditions or a known component of $\vec{B}$ - see sections 17 and 23. For example $\phi$ is constant on the surface of a highly permeable material if the magnetic field is well below saturation, e.g. $B \leq 1.0 T$ for magnetically soft iron. In fact

this formalism is most useful when magnetic materials are present, with $\vec{H}=-\vec{\nabla} \phi$ and $\nabla^{2} \phi=\vec{\nabla} \cdot \vec{M}$ everywhere outside the wire - see sections 16 and 17 . Another difficulty 
with $\phi$ is that it may be multi-valued. This follows from $\oint \vec{B} \cdot \overrightarrow{d r} \neq 0$ around any loop that encloses non-zero net wire current. The idea is to position the discontinuities in the $r-z$ plane such that a single branch of $\phi$ can be applied in the region of interest. Alternatively a hybrid formalism can be used - see section 19.

In vacuum, surfaces with $\phi=$ constant have perpendicular intersection with flux surfaces ( $r A_{\theta}=$ constant), and adjacent surfaces of constant $\phi$ may be specified such that their separation is inversely proportional to $|\vec{B}|$.

\subsection{Global scalar potential}

The limitations of the magnetic scalar potential $\phi$ may be circumvented for a solenoid by defining a global scalar potential, denoted here by $\varphi$. Let

$$
\vec{B}=\mu_{0} S(r, z) \hat{e}_{z}+\vec{\nabla} \varphi
$$

where $S(r, z)$ is the cumulative current distribution defined in section 2 and $J_{\theta}^{\text {mag }}$ can be added to $J_{\theta}$ as usual. This formula for $\vec{B}$ satisfies $\vec{\nabla} x \vec{B}=\mu_{0} \vec{J}$. To make $\vec{\nabla} \cdot \vec{B}=0$ we set

$$
\nabla^{2} \varphi=-\frac{\partial}{\partial z} \mu_{0} S(r, z)
$$

The term $\mu_{0} S(r, z) \hat{e}_{z}$ in eqn. (5.5) is just the previously defined direct field of the system - see section 2 , and $\vec{\nabla} \varphi$ is the residual field (i.e. fringe field, external field, etc.). It is clear from eqn. (5.5) that $\varphi$ is single valued since $\vec{B}-\mu_{0} S(r, z) \hat{e}_{z}$ has vanishing curl everywhere. Also, $\varphi$ is a global solution since eqn. (5.6) is valid over all space. In principle a global scalar potential can be defined for any configuration of currents, but it is not useful unless $\vec{\nabla} x \vec{B}=\mu_{0} \vec{J}$ has a simple solution, as is the case for a solenoidal system.

Note that $\vec{\nabla} \varphi$ is discontinuous at the ends of a magnet bore and it generally subtracts from the direct field inside the bore. This is a very awkward way to treat the field close to the axis, where it is used for beam dynamics calculations and smoothness is important. However, $\varphi$ gives an intuitively appealing picture of the external field by an analogy with electrostatics, with the source on the rhs of eqn. (5.6) acting like a charge density proportional to $\partial S(r, z) / \partial z$. For a thin current layer at radius $\mathrm{R}$, this source is a pair of disks of opposite magnetic charge $\left( \pm \pi R^{2} \mu_{0} S\right)$ at the solenoid ends. In section 11 the global scalar potential will be used to evaluate the external field, which will in turn be used in section 15 to calculate the interference of fields among off-axis solenoids. 


\section{Green Function Solutions}

\subsection{Green function formalism}

Any equation of the form (in 3-d)

$$
\nabla^{2} f=g(\vec{r})
$$

with $g(\vec{r})=0$ at large $|\vec{r}|$, may be formally integrated using the Green function

$$
G\left(\vec{r}, \overrightarrow{r^{\prime}}\right)=\frac{-1}{4 \pi\left|\overrightarrow{r^{\prime}}-\vec{r}\right|} ; \quad f(\vec{r})=\int d^{3} r^{\prime} G\left(\vec{r}, \overrightarrow{r^{\prime}}\right) g\left(\overrightarrow{r^{\prime}}\right)
$$

This works because

$$
\nabla^{2} G=\nabla^{\prime 2} G=\delta\left(\overrightarrow{r^{\prime}}-\vec{r}\right)
$$

where $\delta(\vec{x})$ is the 3 -d Dirac delta function. In general, from eqn. (2.3c),

$$
\vec{B}=\frac{\mu_{0}}{4 \pi} \int d^{3} r^{\prime} \frac{\vec{\nabla}^{\prime} x \vec{J}^{\prime}}{\left|\vec{r}^{\prime}-\vec{r}\right|}
$$

It is seen from eqn. (6.2) that $G\left(\vec{r}, \overrightarrow{r^{\prime}}\right)$ diverges as $\left|\overrightarrow{r^{\prime}}-\vec{r}\right| \rightarrow 0$, but this is not a fundamental difficulty. The Green function solution $f(\vec{r})$ is finite unless $g(\vec{r})$ is itself singular. However, a numerical evaluation of $f(\vec{r})$ in a region where $g(\vec{r}) \neq 0$ may suffer from an unphysical divergence; this is easily fixed by letting $\left|\overrightarrow{r^{\prime}}-\vec{r}\right| \rightarrow\left(\left|\overrightarrow{r^{\prime}}-\vec{r}\right|^{2}+10^{-6}\right)^{1 / 2}$ in the Green function. For a more accurate fix see the comment following eqn. (6.29).

We have already used $G\left(\vec{r}, \overrightarrow{r^{\prime}}\right)$ to solve for $B_{z}$ in section (3):

$$
B_{z}=\frac{\mu_{0}}{4 \pi} \int d^{3} r^{\prime}\left(\frac{1}{r^{\prime}} \frac{\partial}{\partial r^{\prime}} r^{\prime} J_{\theta}^{\prime}\right) \frac{1}{\left|\overrightarrow{r^{\prime}}-\vec{r}\right|}=\frac{\mu_{0}}{4 \pi} \int d^{3} r^{\prime} J_{\theta}^{\prime} \frac{r^{\prime}-r \cos \left(\theta^{\prime}-\theta\right)}{\left|\overrightarrow{r^{\prime}}-\vec{r}\right|^{3}}
$$


Equation (5.6) for the global scalar potential $\varphi$ is also of the proper form for application of the Green function:

$$
\varphi=\frac{\mu_{0}}{4 \pi} \int d^{3} r^{\prime}\left(\frac{\partial S^{\prime}}{\partial z^{\prime}}\right) \frac{1}{\left|\overrightarrow{r^{\prime}}-\vec{r}\right|} .
$$

Equations (2.3b) for $B_{r}$ and (5.3) for $A_{\theta}$ do not have the Laplacian operator, but they may be converted to the desired form by multiplying both sides by $\cos \theta$, i.e.

$$
\nabla^{2}\left(B_{r} \cos \theta\right)=\left(\frac{\partial}{\partial r} \frac{1}{r} \frac{\partial}{\partial r} r B_{r}+\frac{\partial^{2} B_{r}}{\partial z^{2}}\right) \cos \theta=\mu_{0} \frac{J_{\theta}}{\partial z} \cos \theta .
$$

This yields

$$
B_{r}=-\frac{\mu_{0}}{4 \pi} \int d^{3} r^{\prime}\left(\frac{\partial J_{\theta}^{\prime}}{\partial z^{\prime}}\right) \frac{\cos \left(\theta^{\prime}-\theta\right)}{\left|\overrightarrow{r^{\prime}}-\vec{r}\right|},
$$

where we have used the identity

$$
\cos \left(\theta^{\prime}\right)=\cos (\theta) \cos \left(\theta^{\prime}-\theta\right)-\sin (\theta) \sin \left(\theta^{\prime}-\theta\right)
$$

inside the Green function integral (the $\sin (\theta)$ contribution vanishes by symmetry). In similar fashion we get

$$
A_{\theta}=\frac{\mu_{0}}{4 \pi} \int d^{3} r^{\prime} J_{\theta}{ }^{\prime} \frac{\cos \left(\theta^{\prime}-\theta\right)}{\left|\overrightarrow{r^{\prime}}-\vec{r}\right|} .
$$

The four Green function solutions given above all have denominators that are odd powers of the function

$$
\left|\overrightarrow{r^{\prime}}-\vec{r}\right|=\left[\left(z^{\prime}-z\right)^{2}+r^{\prime 2}+r^{2}-2 r^{\prime} r \cos \left(\theta^{\prime}-\theta\right)\right]^{1 / 2} .
$$

Several transformations can be made. First, by symmetry we may let $\theta^{\prime}-\theta \rightarrow \theta^{\prime}$ everywhere in an integrand. Second, if a derivative with respect to $z$ or $z^{\prime}$ appears in the integrand, we may use the identity

$$
\frac{\partial}{\partial z} \frac{1}{\left|\overrightarrow{r^{\prime}}-\vec{r}\right|}=-\frac{\partial}{\partial z^{\prime}} \frac{1}{\left|\overrightarrow{r^{\prime}}-\vec{r}\right|},
$$


integrate by parts, and sometimes get a simplification. For example, a unit step-function of $z^{\prime}$ can be turned into a delta function of $z^{\prime}$.

\subsection{Explicit field expressions}

Writing everything explicitly, we have

$$
B_{z}=\frac{\mu_{0}}{4 \pi} \int_{-\infty}^{\infty} d z^{\prime} \int_{0}^{\infty} d r^{\prime} r^{\prime} J_{\theta}\left(r^{\prime}, z^{\prime}\right) \int_{0}^{2 \pi} d \theta^{\prime} \frac{\left(r^{\prime}-r \cos \left(\theta^{\prime}\right)\right)}{{\sqrt{\left(\mathrm{z}^{\prime}-\mathrm{z}\right)^{2}+r^{\prime 2}+r^{2}-2 r^{\prime} r \cos \left(\theta^{\prime}\right)}}^{3}}
$$

Denoting the denominator in eqn. (6.12) by $\sqrt{\ldots \ldots}^{3}$, we get for the other fields

$$
\begin{aligned}
& B_{r}=-\frac{\mu_{0}}{4 \pi} \int_{-\infty}^{+\infty} d z^{\prime} \int_{0}^{\infty} d r^{\prime} r^{\prime} \frac{\partial J_{\theta}\left(r^{\prime}, z^{\prime}\right)}{\partial z^{\prime}} \int_{0}^{2 \pi} d \theta^{\prime} \frac{\cos \left(\theta^{\prime}\right)}{\sqrt{\ldots .}}, \\
& A_{\theta}=\frac{\mu_{0}}{4 \pi} \int_{-\infty}^{+\infty} d z^{\prime} \int_{0}^{\infty} d r^{\prime} r^{\prime} J_{\theta}\left(r^{\prime}, z^{\prime}\right) \int_{0}^{2 \pi} d \theta^{\prime} \frac{\cos \left(\theta^{\prime}\right)}{\sqrt{\ldots .}} \\
& \varphi=\frac{\mu_{0}}{4 \pi} \int_{-\infty}^{+\infty} d z^{\prime} \int_{0}^{\infty} d r^{\prime} r^{\prime} \frac{\partial S\left(r^{\prime}, z^{\prime}\right)}{\partial z^{\prime}} \int_{0}^{2 \pi} d \theta^{\prime} \frac{1}{\sqrt{\ldots \ldots}}
\end{aligned}
$$

It is clear that if we derive $B_{r}=-\partial A_{\theta} / \partial z$ from eqn. (6.14) we will simply recover the result obtained by direct integration, eqn. (6.13). We may also derive $B_{z}=\frac{1}{r} \frac{\partial}{\partial r} r A_{\theta}$ using eqn. (6.14). This procedure is correct, but it is not obvious from inspection that the result is actually equivalent to the direct integration, eqn. (6.12). We therefore have the alternative form:

$$
\begin{aligned}
B_{z}=\frac{1}{r} & \frac{\partial}{\partial r} r A_{\theta}=\frac{\mu_{0}}{4 \pi} \int_{-\infty}^{+\infty} d z^{\prime} \int_{0}^{\infty} d r^{\prime} r^{\prime} J_{\theta}\left(r^{\prime}, z^{\prime}\right) \bullet \\
& \quad \int_{0}^{2 \pi} d \theta^{\prime} \cos \left(\theta^{\prime}\right)\left[\frac{1}{r \sqrt{\ldots .}}-\frac{r-r^{\prime} \cos \left(\theta^{\prime}\right)}{\sqrt{\ldots .}}\right] .
\end{aligned}
$$

It may be verified from numerical examples that the two formulas for $B_{z}$ are the same. Mathematically, their equivalence can be reduced to the identity (with $0 \leq a \leq 1$ )

$$
\int_{0}^{2 \pi} d \theta \sqrt{1-a \cos (\theta)}=\left(1-a^{2}\right) \int_{0}^{2 \pi} d \theta \frac{1}{\sqrt{1-a \cos (\theta)}^{3}}
$$


which is also not obvious. One proof of eqn. (6.17) is that the two expressions have the same expansion coefficients multiplying all powers of $a^{2}$. This identity will be used in section 7 and below in eqn. (6.25d).

Not surprisingly, there is also a second integral expression for $A_{\theta}$, which can be derived from eqns. (6.5) and (6.9):

$$
\begin{aligned}
& A_{\theta}=r B_{z}-r \frac{\partial A_{\theta}}{\partial r} \\
& =\frac{\mu_{0}}{4 \pi} \int d^{3} r^{\prime} \frac{J_{\theta}^{\prime} r^{\prime} r \sin ^{2}\left(\theta^{\prime}-\theta\right)}{\left|\overrightarrow{r^{\prime}}-\vec{r}\right|^{3}} \\
& =\frac{\mu_{0}}{4 \pi} \int_{-\infty}^{+\infty} d z^{\prime} \int_{0}^{\infty} d r^{\prime} r^{\prime 2} r J_{\theta}\left(r^{\prime}, z^{\prime}\right) \int_{0}^{2 \pi} d \theta^{\prime} \frac{\sin ^{2}\left(\theta^{\prime}\right)}{\sqrt{\ldots .}}
\end{aligned}
$$

Similar to eqn. (6.17), the equivalence of this expression and eqn. (6.14) implies an identity:

$$
\int_{0}^{2 \pi} d \theta \frac{\sin ^{2}(\theta)}{\sqrt{1-a \cos (\theta)}^{3}}=\frac{2}{a} \int_{0}^{2 \pi} d \theta \frac{\cos (\theta)}{\sqrt{1-a \cos (\theta)}}
$$

which can be proved using integration by parts in $\theta$. The two identities can be shown to be essentially equivalent.

\subsection{Reduction to complete elliptic integrals}

Numerical integration of the Green function solutions is greatly simplified when the integrals over $\theta^{\prime}$ are worked in advance, leaving only integrations over $r^{\prime}$ and $z^{\prime}$. This can be done exactly using the complete elliptic integrals $\mathrm{E}$ and $\mathrm{K}$, or in an accurate approximation using analytical fits - see section 7 . First we isolate the essential $\theta^{\prime}$ integrations. Denote

$$
a\left(r, z, r^{\prime}, z^{\prime}\right) \equiv \frac{2 r r^{\prime}}{\left(z^{\prime}-z\right)^{2}+r^{2}+r^{\prime 2}},
$$

which has range $0 \leq a \leq 1$, and define the five functions:

$$
f_{0}(a)=\int_{0}^{2 \pi} \frac{d \theta}{2 \pi} \sqrt{1-a \cos (\theta)}
$$




$$
\begin{aligned}
& f_{1}(a)=\int_{0}^{2 \pi} \frac{d \theta}{2 \pi} \frac{\cos (\theta)}{\sqrt{1-a \cos (\theta)}}, \\
& f_{2}(a)=\int_{0}^{2 \pi} \frac{d \theta}{2 \pi} \frac{1}{\sqrt{1-a \cos (\theta)}}, \\
& f_{3}(a)=\int_{0}^{2 \pi} \frac{d \theta}{2 \pi} \frac{\cos (\theta)}{\sqrt{1-a \cos (\theta)}^{3}}, \\
& f_{4}(a)=\int_{0}^{2 \pi} \frac{d \theta}{2 \pi} \frac{1}{\sqrt{1-a \cos (\theta)}^{3}} .
\end{aligned}
$$

We express $f_{0}$ and $f_{2}$ in terms of $\mathrm{E}$ and $\mathrm{K}$; the standard definitions [6-1] are

$$
\begin{aligned}
& E(x)=\int_{0}^{\pi / 2} d y \sqrt{1-x \sin ^{2}(y)}, \\
& K(x)=\int_{0}^{\pi / 2} d y \frac{1}{\sqrt{1-x \sin ^{2}(y)}} .
\end{aligned}
$$

Warning: these definitions of $\mathrm{E}(x)$ and $K(x)$ are used in Mathematica ${ }^{\circledR}$ [6-2], but in some texts $x^{2}$ appears in the integrands instead of $x$. Making a change of variable in these definitions, we get

$$
\begin{aligned}
& f_{0}(a)=\frac{2 \sqrt{1+a}}{\pi} E\left(\frac{2 a}{1+a}\right), \\
& f_{2}(a)=\frac{2}{\pi \sqrt{1+a}} K\left(\frac{2 a}{1+a}\right),
\end{aligned}
$$

and the remaining $f_{i}(a)$ are found from $f_{0}$ and $f_{2}$ using the relations

$$
\begin{aligned}
& f_{1}(a)=\frac{1}{a}\left(f_{2}(a)-f_{0}(a)\right), \\
& f_{4}(a)=\frac{f_{0}(a)}{1-a^{2}}, \\
& f_{3}(a)=\frac{1}{a}\left(\frac{f_{0}(a)}{1-a^{2}}-f_{2}(a)\right) .
\end{aligned}
$$

The four Green function solutions, eqns. (6.12-6.15), reduce to 


$$
\begin{aligned}
& B_{z}=\frac{\mu_{0}}{2} \int_{-\infty}^{+\infty} d z^{\prime} \int_{0}^{\infty} d r^{\prime} r^{\prime 2} J_{\theta}\left(r^{\prime}, z^{\prime}\right){\sqrt{\frac{a}{2 r r^{\prime}}}}^{3}\left[f_{4}(a)-\frac{r}{r^{\prime}} f_{3}(a)\right], \\
& B_{r}=\frac{-\mu_{0}}{2} \int_{-\infty}^{+\infty} d z^{\prime} \int_{0}^{\infty} d r^{\prime} r^{\prime} \frac{\partial J_{\theta}\left(r^{\prime}, z^{\prime}\right)}{\partial z^{\prime}} \sqrt{\frac{a}{2 r r^{\prime}}} f_{1}(a), \\
& A_{\theta}=\frac{\mu_{0}}{2} \int_{-\infty}^{+\infty} d z^{\prime} \int_{0}^{\infty} d r^{\prime} r^{\prime} J_{\theta}\left(r^{\prime}, z^{\prime}\right) \sqrt{\frac{a}{2 r r^{\prime}}} f_{1}(a), \\
& \varphi=\frac{\mu_{0}}{2} \int_{-\infty}^{+\infty} d z^{\prime} \int_{0}^{\infty} d r^{\prime} r^{\prime} \frac{\partial S\left(r^{\prime}, z^{\prime}\right)}{\partial z^{\prime}} \sqrt{\frac{a}{2 r r^{\prime}}} f_{2}(a) .
\end{aligned}
$$

The divergence of the Green function carries over to the $f_{i}(a)$ as $a \rightarrow 1.0$. This can be fixed up by a substitution such as $a \rightarrow a /\left(1+a^{4} / 10000\right)$.

\section{References}

6-1 Abramowitz, M. and I.A. Stegun, Handbook of Mathematical Functions, Dover, NY (1965).

6-2 Wolfram, S., The Mathematica ${ }^{\circledR}$ Book, $3^{\text {rd }}$ edition, Cambridge University Press (1991).

\section{Approximation of the Green Function Integrals}

The complete elliptic integrals $E$ and $K$, defined in section 6, may be used to evaluate the functions $f_{i}(a)$ that appear in the general Green function solutions for $B_{z}, B_{r}, A_{\theta}$ and $\varphi$. The resulting formulas are not very revealing; one might just as well evaluate the $f_{i}(a)$ numerically from their defining integrals (see plots of $f_{i}(a)$ below). Our objective in this section is to approximate these integrals with elementary functions and thereby aid analysis and speed up computations.

\subsection{Expansion of $f_{i}(a)$ in powers of $a$}

Except for $f_{0}$, which is defined to help evaluate the other integrals, all of the $f_{i}$ diverge as $a \rightarrow 1$, so their power-series expansions are of limited value. However, since the argument of the $f_{i}(a)$, 


$$
a \equiv \frac{2 r r^{\prime}}{\left(z^{\prime}-z\right)^{2}+r^{\prime 2}+r^{2}},
$$

is small when $r$ is either large or small, these expansions are of use in evaluating both the near-axis field and far field. Expanding $\sqrt{1-a \cos (\theta)}$ in the integrands and working the resulting elementary integrals over $\theta$ yields:

$$
\begin{gathered}
f_{0}(a)=\int_{0}^{2 \pi} \frac{d \theta}{2 \pi} \sqrt{1-a \cos (\theta)}=\left(1-\frac{1}{16} a^{2}-\frac{15}{1024} a^{4}-\frac{105}{16384} a^{6} \ldots\right) \\
f_{1}(a)=\int_{0}^{2 \pi} \frac{d \theta}{2 \pi} \frac{\cos (\theta)}{\sqrt{1-a \cos (\theta)}}=\frac{a}{4}\left(1+\frac{15}{32} a^{2}+\frac{315}{1024} a^{4} \cdots\right) \\
f_{2}(a)=\int_{0}^{2 \pi} \frac{d \theta}{2 \pi} \frac{1}{\sqrt{1-a \cos (\theta)}}=\left(1+\frac{3}{16} a^{2}+\frac{105}{1024} a^{4}+\frac{1155}{16384} a^{6}+\cdots\right), \\
f_{3}(a)=\int_{0}^{2 \pi} \frac{d \theta}{2 \pi} \frac{\cos (\theta)}{\sqrt{1-a \cos (\theta)}^{3}}=\frac{3 a}{4}\left(1+\frac{35}{32} a^{2}+\frac{1155}{1024} a^{4}+\cdots\right) \\
f_{4}(a)=\int_{0}^{2 \pi} \frac{d \theta}{2 \pi} \frac{1}{\sqrt{1-a \cos (\theta)}^{3}}=\left(1+\frac{15}{16} a^{2}+\frac{945}{1024} a^{4}+\frac{15015}{16384} a^{6} \cdots\right)
\end{gathered}
$$

The expansion coefficients and plots suggest that $f_{1}$ and $f_{2}$ diverge as Log $\left(1-a^{2}\right)$, while $f_{3}$ and $f_{4}$ diverge as $1 /\left(1-a^{2}\right)$. This behavior is quantified below and accurate approximate formulas are found that are valid throughout the entire range $0 \leq a \leq 1$. 


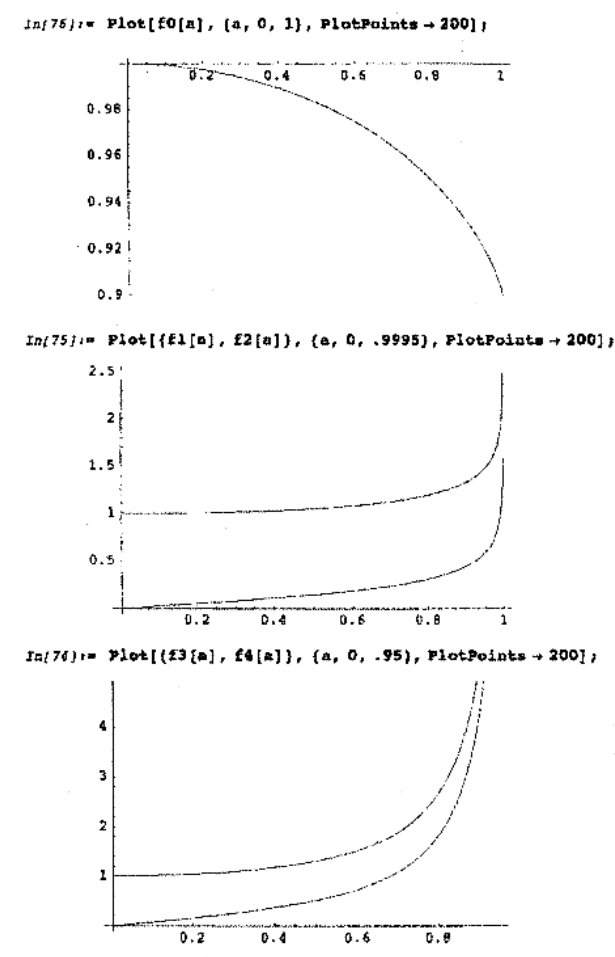

\subsection{Approximation of $f_{i}(a)$ as $a \rightarrow 1$}

To proceed, we make use of five linear relations:

$$
\begin{aligned}
& f_{0}^{\prime}=-f_{1} / 2, \\
& f_{2}^{\prime}=f_{3} / 2, \\
& f_{2}=f_{0}+a f_{1}, \\
& f_{4}=\frac{f_{0}}{1-a^{2}}, \\
& f_{3}=\frac{1}{a}\left(f_{4}-f_{2}\right)=\frac{a f_{0}}{1-a^{2}}-f_{1} .
\end{aligned}
$$

Equations $(7.3 \mathrm{a}, \mathrm{b}, \mathrm{c})$ follow immediately from the definitions of the $f_{i}$, while $(7.3 \mathrm{~d}, \mathrm{e})$ also make use of eqn. (6.17). These five relations determine the $f_{i}$ exactly, given the initial values $\left[f_{0}(0)=1, f_{1}(0)=0\right]$. Consequently, no approximate formulas can satisfy all five relations and end points at once. When we find good approximations for $f_{0}$ and $f_{1}$, 
then $\left(f_{2}, f_{3}, f_{4}\right)$ can be approximated using eqns. (7.3c-e). By eliminating $\left(f_{2}, f_{3}, f_{4}\right)$ among the five relations we obtain coupled first order equations for $f_{0}$ and $4 a f_{1}$ :

$$
\begin{aligned}
& \frac{d}{d a}\left(4 a f_{1}\right)=\frac{2 a f_{0}}{1-a^{2}}, \\
& \frac{d f_{0}}{d a}=-\frac{\left(4 a f_{1}\right)}{8 a} .
\end{aligned}
$$

The value of $f_{0}$ at $a=1$ is obtained from its definition by a simple integration. This number appears repeatedly in expansions of the $f_{i}$ near $a=1$, so it is denoted here by $C$ :

$$
C \equiv f_{0}(1)=\int_{0}^{2 \pi} \frac{d \theta}{2 \pi} \sqrt{1-\cos (\theta)}=\frac{2 \sqrt{2}}{\pi}=.900316316 \ldots
$$

It is seen from the above plot that $f_{0}$ varies only moderately with $a^{2}$, so we have a rough first approximation that recovers the end points:

$$
f_{0} \approx 1-a^{2}+C a^{2}
$$

which has maximum relative error $f_{0}^{\text {approx }} / f_{0}-1=-.0159$ occurring at $a=.823$. This simple fit may be inserted in equation (7.4a) and integrated to get

$$
f_{1} \approx \frac{1}{4 a}\left\{a^{2}-C\left[\log \left(1-a^{2}\right)+a^{2}\right]\right\}
$$

which has the correct leading term as $a \rightarrow 0$ and correct divergence as $a \rightarrow 1$. Its maximum relative error is $\left(f_{1}^{\text {aprox }} / \mathrm{f}_{1}-1\right)=-.0128$ at $a=.931$. To improve on these rough approximations we could evaluate $f_{0}$ by integrating eqn. (7.4b), inserting expression (7.7) for $f_{1}$. This leads to a very accurate formula, but it involves the relatively unfamiliar function PolyLog $\left[2, a^{2}\right]$, which violates the goal of transparency.

We return to the integral that defines $f_{1}$ for guidance. After some analytical effort it is found that as $a \rightarrow 1$,

$$
4 a f_{1}=4 a \int_{0}^{2 \pi} \frac{d \theta}{2 \pi} \frac{\cos (\theta)}{\sqrt{1-a \cos (\theta)}} \rightarrow C\left[D-\log \left(1-a^{2}\right)\right]
$$

where $\mathrm{D}$ is the constant

$$
D \equiv 6 \log (2)-4=.158883084 \ldots
$$


(a beer for any reader who analytically verifies eqns. $(7.8,7.9)$ ). A simple modification of eqn. (7.7) that contains the correct limits for both $a \rightarrow 0$ and $a \rightarrow 1$, is

$$
f_{1}(a) \approx \frac{C}{4 a}\left[\left(\frac{1}{C}-1\right) a^{2}+\left(1+D-\frac{1}{C}\right) a^{4}-\log \left(1-a^{2}\right)\right]
$$

which has maximum relative error $\left(f_{1}^{a p p r o x} / f_{1}-1\right)=.00726$ at $a=.744$. In similar fashion we improve on the rough approximation for $f_{0}$, eqn. (7.6):

$$
f_{0}(a) \approx 1-\frac{a^{2}}{16}-\left(1-C-\frac{1}{16}\right) a^{4}
$$

which has maximum relative error $\left(f_{0}^{\text {approx }} / f_{0}-1\right)=-.00841$ at $a=.902$.

While eqns. (7.10 and 7.11) are adequate for some applications, much more accurate approximations for $f_{1}$ and $f_{0}$ can be derived by including additional terms from their $a \rightarrow 1$ expansions. To treat this limit we first define the variable $x \equiv 1-a^{2}$, so eqns. $(7.4 \mathrm{a}, \mathrm{b})$ can be written in the convenient form

$$
\begin{aligned}
& f_{0}=-x \frac{d}{d x}\left(4 a f_{1}\right), \\
& \left(4 a f_{1}\right)=16(1-x) \frac{d f_{0}}{d x} .
\end{aligned}
$$

Guided by eqn. (7.8), the expanded form of $4 a f_{1}$ is assumed to be

$$
4 a f_{1}=C\left\{\left[D-A_{1} x-A_{2} x^{2}-A_{3} x^{3}-. . .\right]-\left[1-B_{1} x-B_{2} x^{2}-B_{3} x^{2}-. .\right] \log (x)\right\},
$$

where $A_{i}$ and $B_{i}$ are constants to be determined. From eqn. (7.12) we then get

$$
\begin{aligned}
& f_{0}=C\left\{\left[1+\left(A_{1}-B_{1}\right) x+\left(2 A_{2}-B_{2}\right) x^{2}+\left(3 A_{3}-B_{3}\right) x^{3}+. . .\right]-\right. \\
& \left.\left[B_{1} x+2 B_{2} x^{2}+3 B_{3} x^{3}+. .\right] \log (\mathrm{x})\right\}
\end{aligned}
$$

The expansions (7.14) and (7.15) may then be inserted into eqn. (7.13) and the coefficients of terms of the form $x^{n}$ or $x^{n} \log (x)$ equated. This somewhat laborious procedure yields for the first four values of $B_{i}$ and $A_{i}$ :

$$
B_{1}=\frac{1}{16}=.0625, \quad B_{2}=\frac{15}{1024}=.014648438 \ldots,
$$




$$
\begin{gathered}
B_{3}=\frac{105}{16384}=.006408691 \ldots, B_{4}=\frac{15015}{4194304}=.003579855 \ldots, \quad(7.16 \mathrm{c}, \mathrm{d}) \\
A_{1}=\frac{2+D}{16}=.134930193 \ldots, A_{2}=\frac{13+15 D}{1024}=.015022701 \ldots, \quad(7.16 \mathrm{e}, \mathrm{f}) \\
A_{3}=\frac{163+315 D}{49152}=.004334476 \ldots, A_{4}=\frac{31183+90090 D}{25165824}=.001807879 \ldots .(7.16 \mathrm{~g}, \mathrm{~h})
\end{gathered}
$$

\subsection{Accurate approximation for $0 \leq a \leq 1$}

We now have two expansions each for $f_{0}$ and $f_{1}$, which apply as either $a \rightarrow 0$ or $a \rightarrow 1$. The goal is to combine terms from these expansions such that simple, accurate formulas are obtained for the entire interval $(0 \leq a \leq 1)$. There are many ways to do this and just one is described here. That is to retain only the terms $A_{1}$ and $B_{1}$ from the expansions that are valid as $a \rightarrow 1$, and insert additional terms that recover the correct limits as $a \rightarrow 0$. These inserted terms vanish as $\left(1-a^{2}\right)^{2}$ when $a \rightarrow 1$ and include parameters that can be adjusted to produce a minimum error over the whole interval.

Denoting the approximate formulas as $g_{0}(a)$ and $g_{1}(a)$, we first set

$$
\begin{aligned}
& f_{1}(a) \approx g_{1}(a)=\frac{C}{4 a}\left\{\left[D-A_{1}\left(1-a^{2}\right)-p\left(1-a^{2}\right)^{2}\left(1+q a^{4}\right)\right]\right. \\
& \left.-\left[1-B_{1}\left(1-a^{2}\right)-r\left(1-a^{2}\right)^{2}\left(1+s a^{4}\right)\right] \log \left(1-a^{2}\right)\right\} .
\end{aligned}
$$

To recover the terms $4 a f_{1} \rightarrow 0+a^{2}+15 a^{4} / 32$ as $a \rightarrow 0$ we set

$$
\begin{aligned}
& p=D-A_{1}=.023952890 \ldots, \\
& r=-\frac{1}{C}+A_{1}+2 p+1-B_{1}=.009615239 \ldots, \\
& q=\frac{1}{p}\left(-\frac{15}{32 C}-p+\frac{1+B_{1}+3 r}{2}\right)=.044655330 \ldots,
\end{aligned}
$$

and for an overall optimum approximation

$$
s=.141 \quad .
$$

This gives the maximum relative error 


$$
\frac{g_{1}}{f_{1}}-1=\mp 8.5 \times 10^{-6}
$$

at $a=.57$ and .89 .

For $f_{0}(a)$ we have

$$
\begin{aligned}
f_{0}(a) \approx g_{0}(a)= & C\left\{\left[1+\left(A_{1}-B_{1}\right)\left(1-a^{2}\right)+t\left(1-a^{2}\right)^{2}\left(1+u a^{4}\right)\right]\right. \\
& \left.-\left[B_{1}\left(1-a^{2}\right)+v\left(1-a^{2}\right)^{2}\left(1+w a^{4}\right)\right] \log \left(1-a^{2}\right)\right\} .
\end{aligned}
$$

To recover the terms $f_{0} \rightarrow 1-a^{2} / 16-15 a^{4} / 1024$ as $a \rightarrow 0$ we set

$$
\begin{aligned}
& t=\frac{1}{C}-1-A_{1}+B_{1}=.038290541 \ldots, \\
& v=-\frac{1}{16 C}-2 B_{1}+A_{1}+2 t=.017091230 \ldots, \\
& u=\frac{1}{t}\left(-\frac{15}{1024 C}-t+\frac{B_{1}+3 v}{2}\right)=.060745565 \ldots,
\end{aligned}
$$

and for an overall optimum approximation

$$
w=.205 \text {. }
$$

This gives maximum relative error

$$
\frac{g_{0}}{f_{0}}-1= \pm 2.4 \times 10^{-5}
$$

at $a=.70, .94$.

In numerical form these approximate functions are

$$
\begin{aligned}
& g_{1}(a)=\frac{1}{4 a}\left(\frac{1}{1.1107207}\right)\left\{\left[(.1588831)-(.1349302)\left(1-a^{2}\right)-\right.\right. \\
& \left.-(.0239529)\left(1-a^{2}\right)^{2}\left(1+(.0446553) a^{4}\right)\right]-[1- \\
& \left.\left.-(.0625)\left(1-a^{2}\right)-(.0096153)\left(1-a^{2}\right)^{2}\left(1+(.141) a^{4}\right)\right] \log \left(1-a^{2}\right)\right\},
\end{aligned}
$$




$$
\begin{aligned}
& g_{0}(a)=\left(\frac{1}{1.1107207}\right)\left\{\left[1+(.0724302)\left(1-a^{2}\right)+(.0382905) \bullet\right.\right. \\
& \left.\cdot\left(1-a^{2}\right)^{2}\left(1+(.0607456) a^{4}\right)\right]-\left[(.0625)\left(1-a^{2}\right)+\right. \\
& \left.\left.+(.0170912)\left(1-a^{2}\right)^{2}\left(1+(.205) a^{4}\right)\right] \log \left(1-a^{2}\right)\right\} .
\end{aligned}
$$

\subsection{Systematic approximation of $f_{i}(a)$}

The optimized fits for $f_{0}(a)$ and $f_{1}(a)$ given above by eqns. $(7.23,7.24)$, are sufficient for essentially any numerical evaluation of ideal solenoidal fields in the absence of magnetic materials. However, it is also useful to have formulas for the $f_{i}(a)$ that have the correct expansions in powers of $a$ through any specified order, even though there may be minor inaccuracies near $a=1$. This situation arises, for example, in evaluating the dynamical effects of fringe field aberrations. The approximate formulas given below are each composed from terms that adequately reproduce $f_{i}(a)$ as $a \rightarrow 1$, followed by a polynomial with three coefficients that successively reproduce the first three expansion terms as $a \rightarrow 0$ (for $f_{1}$ and $f_{3}$ the first term vanishes). For example, we set

$$
f_{0}(a) \approx h_{0}(a)=C\left\{\left[1+\frac{1+D}{16}\left(1-a^{2}\right)-\frac{1}{16}\left(1-a^{2}\right) \log \left(1-\mathrm{a}^{2}\right)\right]+\left[\alpha+\beta a^{2}+\gamma a^{4}\right]\left(1-a^{2}\right)^{2}\right\} .
$$

The coefficients $(\alpha, \beta, \gamma)$ are fixed to successively recover the three leading terms $f_{0} \rightarrow 1-\frac{1}{16} a^{2}-\frac{15}{1024} a^{4}$. If one doesn't care about the $a^{4}$ term, the coefficient $\gamma$ can be dropped without changing $\alpha$ or $\beta$. Similarly an additional term $\sim a^{6}$ could be added to the polynomial if a correct sixth order term in $h_{0}$ is desired. The systematic formulas are as follows:

$$
\begin{aligned}
& f_{0}(a) \approx h_{0}(a)=C\left\{\left[1+\frac{1+D}{16}\left(1-a^{2}\right)-\frac{1}{16}\left(1-a^{2}\right) \log \left(1-\mathrm{a}^{2}\right)\right]\right. \\
& \left.+\left[\left(\frac{1}{C}-\frac{17}{16}-\frac{D}{16}\right)+\left(\frac{31}{16 C}-\frac{34}{16}-\frac{D}{16}\right) a^{2}+\left(\frac{2929}{1024 C}-\frac{101}{32}-\frac{D}{16}\right) a^{4}\right]\left(1-a^{2}\right)^{2}\right\},
\end{aligned}
$$

with maximum relative error $h_{0} / f_{0}-1=-.00055$ at $a=.83$, 


$$
\begin{aligned}
& f_{1}(a) \approx h_{1}(a)=\frac{C}{4 a}\left\{\left[D-\frac{2+D}{16}\left(1-a^{2}\right)-\log \left(1-\mathrm{a}^{2}\right)+\frac{1}{16}\left(1-a^{2}\right) \log \left(1-\mathrm{a}^{2}\right)\right]\right. \\
& \left.+\left[\left(\frac{2}{16}-\frac{15 D}{16}\right)+\left(\frac{1}{C}-\frac{13}{16}-\frac{31 D}{16}\right) a^{2}+\left(\frac{79}{32 C}-\frac{73}{32}-\frac{47 D}{16}\right) a^{4}\right]\left(1-a^{2}\right)^{2}\right\}
\end{aligned}
$$

with maximum relative error $h_{1} / f_{1}-1=.00029$ at $a=.73$,

$$
\begin{aligned}
& f_{2}(a) \approx h_{2}(a)=h_{0}(a)+a h_{1}(a) \\
& =C\left\{\left[\frac{4+D}{4}+\frac{2+3 D}{64}\left(1-a^{2}\right)-\frac{1}{4} \log \left(1-a^{2}\right)-\frac{3}{64}\left(1-a^{2}\right) \log \left(1-a^{2}\right)\right]\right. \\
& \left.+\left[\left(\frac{1}{C}-\frac{33}{32}-\frac{19 D}{64}\right)+\left(\frac{35}{16 C}-\frac{149}{64}-\frac{35 D}{64}\right) a^{2}+\left(\frac{3561}{1024 C}-\frac{477}{128}-\frac{51 D}{64}\right) a^{4}\right]\left(1-a^{2}\right)^{2}\right\},
\end{aligned}
$$

with maximum relative error $\frac{h_{2}}{f_{2}}-1=-.00037$ at $a=.815$,

$$
\begin{aligned}
& f_{3}(a) \approx h_{3}(a)=\frac{C}{a}\left\{\left[\frac{1}{1-a^{2}}-\frac{15+3 D}{16}+\frac{3}{16} \log \left(1-a^{2}\right)\right]\right. \\
& \left.+\left[\left(\frac{3 D-1}{16}\right)+\left(\frac{3}{4 C}-\frac{14}{16}+\frac{3 D}{16}\right) a^{2}+\left(\frac{201}{128 C}-\frac{57}{32}+\frac{3 D}{16}\right) a^{4}\right]\left(1-a^{2}\right)\right\},
\end{aligned}
$$

with maximum relative error $\frac{h_{3}}{f_{3}}-1=.00064$ at $a=.77$, and finally

$$
\begin{aligned}
& f_{4}(a) \approx h_{4}(a)=\frac{h_{0}(a)}{1-a^{2}}=C\left\{\left[\frac{1}{1-a^{2}}+\frac{1+D}{16}-\frac{1}{16} \log \left(1-a^{2}\right)\right]\right. \\
& \left.+\left[\left(\frac{1}{C}-\frac{17}{16}-\frac{D}{16}\right)+\left(\frac{31}{16 C}-\frac{34}{16}-\frac{D}{16}\right) a^{2}+\left(\frac{2929}{1024 C}-\frac{101}{32}-\frac{D}{16}\right) a^{4}\right]\left(1-a^{2}\right)\right\}
\end{aligned}
$$

with maximum relative error $\frac{h_{4}}{f_{4}}-1=-.00055$ at $a=.83$.

\section{Thin Current Layer - Complete Solution}

This section presents many expressions, both exact and approximate, for the fields of a uniform, thin current layer. In most cases the current layer extends from $z=-\infty$ to $z=0$; the fields produced by layers of finite length may then be found by translation and superposition. 


\subsection{Semi-infinite layer: near-axis field}

In section 4 we derived the on-axis field for a thin, semi-infinite current layer, which was then used to build up more complex layouts:

$$
\begin{aligned}
& J_{\theta}(r, z)=S \delta(r-R) \Theta(z), \\
& B_{0}(z)=\frac{\mu_{0} S}{2}\left(1-\frac{z}{\sqrt{z^{2}+R^{2}}}\right),
\end{aligned}
$$

where $\Theta(z)$ is the downward unit step defined in section 2. From section 3 the near-axis field components, including the two lowest-order fringe field aberrations, are

$$
\begin{aligned}
& B_{z} \approx B_{0}-\frac{B_{0}^{\prime \prime} r^{2}}{4}+\frac{B_{0}^{i v} r^{4}}{64}-\ldots \\
& =\frac{\mu_{0} S}{2}\left[1-\frac{z}{{\sqrt{z^{2}+R^{2}}}^{2}}-\frac{3}{4} \frac{R^{2} z r^{2}}{{\sqrt{z^{2}+R^{2}}}^{5}}-\frac{15}{64} \frac{R^{2}\left(3 R^{2} z-4 z^{3}\right) r^{4}}{{\sqrt{z^{2}+R^{2}}}^{9}}+\ldots\right], \\
& B_{r}=-\frac{B_{0}{ }^{\prime} r}{2}+\frac{B_{0}^{\prime \prime \prime} r^{3}}{16}-\frac{B_{0}^{v} r^{5}}{384}+\ldots \\
& =\frac{\mu_{0} S}{4}\left[\frac{R^{2} r}{{\sqrt{z^{2}+R^{2}}}^{3}}+\frac{3}{8} \frac{R^{2}\left(R^{2}-4 z^{2}\right) r^{3}}{{\sqrt{z^{2}+R^{2}}}^{7}}+\frac{15}{64} \frac{R^{2}\left(R^{4}-12 z^{2} R^{2}+8 z^{4}\right) r^{5}}{{\sqrt{z^{2}+R^{2}}}^{11}}+\ldots\right] \text {. }
\end{aligned}
$$

\subsection{Semi-infinite layer: exact fields and potentials}

The near-axis fields of the semi-infinite layer may be extended to any value of $r$ using the Green function solutions. The resulting expressions involve only integrations over $\theta$ because the integrations over $r^{\prime}$ and $z^{\prime}$ are workable. We have from eqns. (6.12) and (6.13):

$$
\begin{array}{r}
B_{z}=\frac{\mu_{0}}{4 \pi} \int_{-\infty}^{+\infty} d z^{\prime} \int_{0}^{\infty} d r^{\prime} r^{\prime}\left[S \delta\left(r^{\prime}-R\right) \Theta\left(z^{\prime}\right)\right] \int_{0}^{2 \pi} d \theta \frac{r^{\prime}-r \cos (\theta)}{{\sqrt{\left(z^{\prime}-z\right)^{2}+r^{\prime 2}+r^{2}-2 r^{\prime} r \cos (\theta)}}^{3}} \\
=\frac{\mu_{0} S}{2} \int_{0}^{2 \pi} \frac{d \theta}{2 \pi}\left(\frac{R^{2}-R r \cos (\theta)}{R^{2}+r^{2}-2 R r \cos (\theta)}\right)\left[1-\frac{z}{\sqrt{z^{2}+R^{2}+r^{2}-2 R r \cos (\theta)}}\right],
\end{array}
$$




$$
\begin{gathered}
B_{r}=\frac{-\mu_{0}}{4 \pi} \int_{-\infty}^{\infty} d z^{\prime} \int_{0}^{\infty} d r^{\prime} r^{\prime}\left[\frac{\partial}{\partial z^{\prime}} S \delta\left(r^{\prime}-R\right) \Theta\left(z^{\prime}\right)\right] \int_{0}^{2 \pi} d \theta \frac{\cos (\theta)}{\sqrt{\left(z^{\prime}-z\right)^{2}+r^{\prime 2}+r^{2}-2 r^{\prime} r \cos (\theta)}} \\
=\frac{\mu_{0} S}{2} \int_{0}^{2 \pi} \frac{d \theta}{2 \pi} \frac{R \cos (\theta)}{\sqrt{z^{2}+R^{2}+r^{2}-2 R r \cos (\theta)}} .
\end{gathered}
$$

The first integral in eqn. (8.4a) for $B_{z}$ is simply (but not obviously)

$$
\int_{0}^{2 \pi} \frac{d \theta}{2 \pi} \frac{R^{2}-R r \cos (\theta)}{R^{2}+r^{2}-2 R r \cos (\theta)}=\Theta(r-R)
$$

so we find the expected limits

$$
B_{z} \rightarrow \begin{cases}\mu_{0} S \Theta(r-R) & z \rightarrow-\infty \\ 0 & z \rightarrow+\infty\end{cases}
$$

while at $z=0$ there is the neat result:

$$
B_{z}(r, 0)=\frac{\mu_{0} S}{2} \Theta(r-R)
$$

For $|\vec{r}| \gg>$, eqns. (8.4a,b) may be expanded in inverse powers of $|\vec{r}|$ to yield in leading order

$$
\vec{B} \approx \mu_{0} S \Theta(r-R) \Theta(z) \hat{e}_{z}+\frac{\mu_{0} S R^{2} \vec{r}}{4|\vec{r}|^{3}} .
$$

This is just a uniform direct field that terminates at $z=0$ plus a residual "magnetic monopole field" with net outward flux $\pi R^{2} \mu_{0} S$ centered at $\vec{r}=0$. A generalization of this result is presented in section 11. An approximation of $\vec{B}$ for the thin layer, that uses only elementary functions and which is good for all $(r, z)$, is presented at the end of this section.

Eqn. (8.2) for $B_{0}(z)$ is recovered from eqn. (8.4a) by setting $r=0$. For general $(r, z), B_{z}$ does not appear to be expressible in terms of well-known functions, but if we insert $r=R$, then we obtain

$$
B_{z}(R, z)=\frac{\mu_{0} S}{4}\left[1-\frac{z f_{2}\left(2 R^{2} /\left(z^{2}+2 R^{2}\right)\right)}{\sqrt{z^{2}+2 R^{2}}}\right] .
$$


For $z<0$ this is the longitudinal field along the center of the thin current layer. The longitudinal field immediately inside or outside the layer (denoted $r=R \pm$ ) is therefore

$$
B_{z}(R \pm, z<0)=\frac{\mu_{0} S}{4}\left[1-\frac{z f_{2}\left(2 R^{2} /\left(z^{2}+R^{2}\right)\right)}{\sqrt{z^{2}+2 R^{2}}}\right] \mp \frac{\mu_{0} S}{2}
$$

with $f_{2}$ defined in section (7):

$$
f_{2}(a)=\int_{0}^{2 \pi} \frac{d \theta}{2 \pi} \frac{1}{\sqrt{1-a \cos (\theta)}} .
$$

For the vector potential we get from eqn. (6.20):

$$
\begin{gathered}
A_{\theta}=\frac{\mu_{0}}{4 \pi} \int_{-\infty}^{+\infty} d z^{\prime} \int_{0}^{\infty} d r^{\prime} r^{\prime 2} r\left[S \delta\left(r^{\prime}-R\right) \Theta\left(z^{\prime}\right)\right] \int_{0}^{2 \pi} d \theta \frac{\sin ^{2}(\theta)}{\sqrt{\left(z^{\prime}-z\right)^{2}+r^{\prime 2}+r^{2}-2 r^{\prime} r \cos (\theta)}} \\
=\frac{\mu_{0} S R^{2} r}{2} \int_{0}^{2 \pi} \frac{d \theta}{2 \pi} \sin ^{2}(\theta) \int_{-\infty}^{0} d z^{\prime} \frac{1}{\sqrt{\left(z^{\prime}-z\right)^{2}+R^{2}+r^{2}-2 R r \cos (\theta)}} \\
=\frac{\mu_{0} S R^{2} r}{2} \int_{0}^{2 \pi} \frac{d \theta}{2 \pi} \frac{\sin ^{2}(\theta)}{R^{2}+r^{2}-2 R r \cos (\theta)}\left[1-\frac{z}{\sqrt{z^{2}+r^{2}+R^{2}-2 R r \cos (\theta)}}\right] .
\end{gathered}
$$

Finally, for the global potential we get from eqn. (6.15):

$$
\begin{aligned}
\varphi=\frac{\mu_{0}}{4 \pi} \int_{-\infty}^{+\infty} d z^{\prime} \int_{0}^{\infty} d r^{\prime} r^{\prime}\left[\frac{\partial}{\partial z^{\prime}} S \Theta\left(r^{\prime}-R\right) \Theta\left(z^{\prime}\right)\right] \int_{0}^{2 \pi} d \theta \frac{1}{\sqrt{\left(z^{\prime}-z\right)^{2}+r^{\prime 2}+r^{2}-2 r^{\prime} r \cos (\theta)}} \\
=-\frac{\mu_{0} S}{2} \int_{0}^{2 \pi} \frac{d \theta}{2 \pi}\left\{\sqrt{z^{2}+r^{2}+R^{2}-2 R r \cos (\theta)}-\sqrt{z^{2}+r^{2}}\right. \\
\left.+r \cos (\theta) \log \left[\frac{\sqrt{z^{2}+r^{2}+R^{2}-2 R r \cos (\theta)}+R-r \cos (\theta)}{\sqrt{z^{2}+r^{2}}-r \cos (\theta)}\right]\right\} .
\end{aligned}
$$

\subsection{Current layer end divergence}

From equations (8.9) and (8.10) it follows that very close to the current layer end $(r=R \pm, \quad z=0 \pm), B_{z}$ is discontinuous as: 


\begin{tabular}{|c|c|c|}
\hline$r$ & $z$ & $B_{z} / \mu_{0} S$ \\
\hline$R-$ & $0-$ & $3 / 4$ \\
\hline$R-$ & $0+$ & $1 / 4$ \\
\hline$R+$ & $0-$ & $-1 / 4$ \\
\hline$R+$ & $0+$ & $1 / 4$ \\
\hline
\end{tabular}

This peculiar behavior is easily seen by numerically evaluating expression (8.4a) near the current layer end. It can also be deduced from the divergent behavior of $B_{r}$ (see below), using the Maxwell equation $\vec{\nabla} \cdot \vec{B}=0$.

Expression (8.4b) for $B_{r}$ can be written in the compact form

$$
\begin{aligned}
& B_{r}=\frac{\mu_{0} S}{2} \frac{R}{\sqrt{z^{2}+r^{2}+R^{2}}} f_{1}(b), \\
& b \equiv 2 R r /\left(z^{2}+r^{2}+R^{2}\right), \\
& f_{1}(b)=\int_{0}^{2 \pi} \frac{d \theta}{2 \pi} \frac{\cos (\theta)}{\sqrt{1-b \cos (\theta)}},
\end{aligned}
$$

as defined in section 6 . Recall from section 7 that as $b \rightarrow 1$,

$$
f_{1}(b) \rightarrow \frac{C}{4 b}\left[D-\log \left(1-b^{2}\right)\right]
$$

where $C=2 \sqrt{2} / \pi$ and $D=(6 \log (2)-4)$. It is apparent that $B_{r}$ is weakly divergent at $(r=R, z=0)$. Explicitly, around this point

$$
B_{r} \rightarrow \frac{\mu_{0} S}{4 \pi}\left\{D-\log \left[\frac{z^{2}+(r-R)^{2}}{R^{2}}\right]\right\}
$$

Although $B_{r}$ becomes infinite for an infinitesimally thin current layer, it is finite for a layer of finite thickness. In fact in a thin layer $B_{r}$ exceeds the scale field strength $\left(\mu_{0} S\right)$ only when $\sqrt{z^{2}+(r-R)^{2}} \leq R / 400$. For current layer thickness greater than $R / 20$ the divergent behavior of the thin layer is not representative. Interestingly, the logarithmic divergence does have an effect in permanent magnets, which have essentially zero surface current layer thickness; this causes a partial demagnetization at magnet corners due to the non-ideal magnetization properties of the material. 


\subsection{Finite length lens}

For a solenoid of finite length $\ell$ and wire radius $R$, centered at $z=0$, equations $(8.4 \mathrm{a}, \mathrm{b})$ give by translation and superposition:

$$
\begin{aligned}
& B_{z}=\frac{\mu_{0} S}{2} \int_{0}^{2 \pi} \frac{d \theta}{2 \pi}\left[\frac{R^{2}-R r \cos (\theta)}{R^{2}+r^{2}-2 R r \cos (\theta)}\right] \\
& \text { - }\left[\frac{(z+\ell / 2)}{\sqrt{(z+\ell / 2)^{2}+r^{2}+R^{2}-2 R r \cos (\theta)}}-\frac{(z-\ell / 2)}{\sqrt{(z-\ell / 2)^{2}+r^{2}+R^{2}-2 R r \cos (\theta)}}\right] \\
& B_{r}=\frac{\mu_{0} S}{2} \int_{0}^{2 \pi} \frac{d \theta}{2 \pi} \bullet \\
& \text { - }\left[\frac{R \cdot 17 \mathrm{a})}{\sqrt{(\mathrm{z}-\ell / 2)^{2}+r^{2}+R^{2}-2 R r \cos (\theta)}}-\frac{R \cos (\theta)}{\sqrt{(\mathrm{z}+\ell / 2)^{2}+r^{2}+R^{2}-2 R r \cos (\theta)}}\right]
\end{aligned}
$$

Plots of $B_{z}$ and $B_{r}$ for several values of $r$ are displayed below for a lense of length $\ell=4.0 \mathrm{~m}$ and radius $R=1.0 \mathrm{~m}$. Note that the plots of $B_{z}$ "square up" for $r \approx R$, while the plots of $B_{r}$ display the logarithmic divergence. 


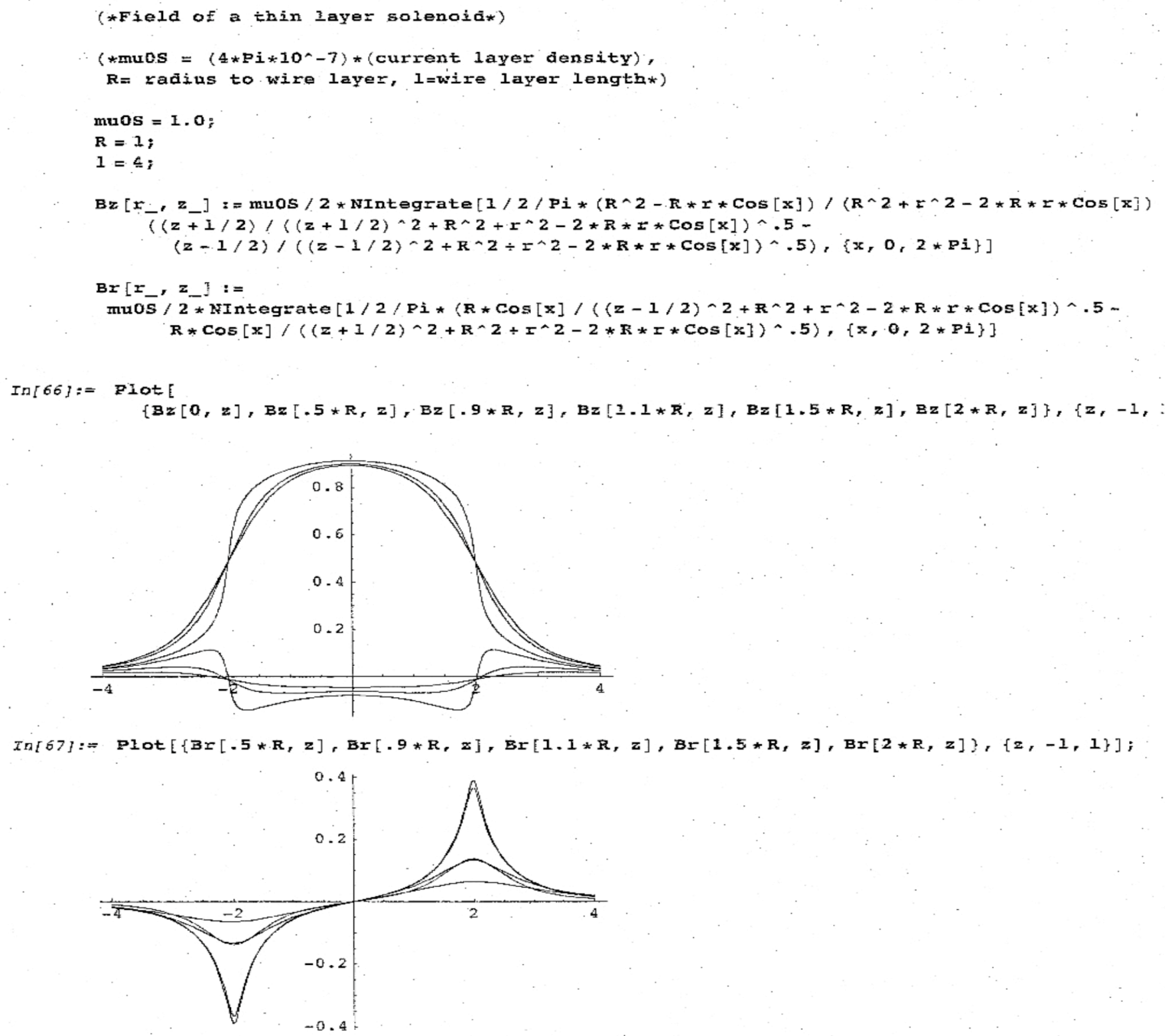

The vector potential of the finite length, thin layer, solenoid can also be written as a single integration over $\theta$ using the Green function (6.9):

$$
\begin{aligned}
& A_{\theta}=\frac{\mu_{0}}{4 \pi} \int d^{3} r^{\prime} \frac{J_{\theta}{ }^{\prime} \cos \left(\theta^{\prime}-\theta\right)}{\mid \overrightarrow{r^{\prime}-\vec{r} \mid}} \\
& =\frac{\mu_{0} S R}{2} \int_{0}^{2 \pi} \frac{d \theta}{2 \pi} \cos (\theta) \int_{-\ell / 2}^{\ell / 2} d z^{\prime} \frac{1}{\sqrt{\left(z^{\prime}-z\right)^{2}+r^{2}+R^{2}-2 R r \cos (\theta)}} \\
& =\frac{\mu_{0} S R}{2} \int_{0}^{2 \pi} \frac{d \theta}{2 \pi} \cos (\theta) \log \left[\frac{\sqrt{(z-\ell / 2)^{2}+R^{2}+r^{2}-2 R r \cos (\theta)}-(z-\ell / 2)}{\sqrt{(z+\ell / 2)^{2}+R^{2}+r^{2}-2 R r \cos (\theta)}-(z+\ell / 2)}\right] .
\end{aligned}
$$


Equation (8.11) gives the alternative, equivalent form

$$
\begin{aligned}
& A_{\theta}=\frac{\mu_{0} S R^{2} r}{2} \int_{0}^{2 \pi} \frac{d \theta}{2 \pi} \frac{\sin ^{2}(\theta)}{R^{2}+r^{2}-2 \operatorname{Rrcos}(\theta)} \bullet \\
& \cdot\left[\frac{(z+\ell / 2)}{\sqrt{(z+\ell / 2)^{2}+R^{2}+r^{2}-2 R r \cos (\theta)}}-\frac{(z-\ell / 2)}{\sqrt{(z-\ell / 2)^{2}+R^{2}+r^{2}-2 \operatorname{Rrcos}(\theta)}}\right] .
\end{aligned}
$$

\subsection{Stored energy}

The magnetic field energy of a thin-layer lens is readily evaluated. In the absence of permeable materials we have in general,

$$
W=\int d^{3} r \frac{|\vec{B}|^{2}}{2 \mu_{0}}=\int d^{3} r \frac{\vec{B} \cdot \vec{\nabla} x \vec{A}}{2 \mu_{0}}=\int d^{3} r \frac{\vec{A} \cdot \vec{\nabla} x \vec{B}}{2 \mu_{0}}=\int d^{3} r \frac{\vec{A} \cdot \vec{J}}{2}
$$

For $J_{\theta}=S(z) \delta(r-R)$, this becomes

$$
W=\pi R \int d z S(z) A_{\theta}(R, z)
$$

Inserting expression (8.19) for $A_{\theta}$ and working the integration over $z$ gives for a lens of length $\ell$ :

$$
W=\frac{\pi R^{2} \ell\left(\mu_{0} S\right)^{2}}{2 \mu_{0}} w(x)
$$

where $x=\ell^{2} / 2 R^{2}$ and

$$
w(x)=\frac{1}{\sqrt{x}} \int_{0}^{2 \pi} \frac{d \theta}{2 \pi}(1+\cos (\theta))[\sqrt{x+1-\cos (\theta)}-\sqrt{1-\cos (\theta)}] .
$$

This function can be expressed using the previously defined $f_{0}$ and $f_{3}$-see section 7: 


$$
w(x)=\sqrt{\frac{x+1}{x}}\left[f_{0}\left(\frac{1}{1+x}\right)-\frac{1}{3}\left(1-\frac{1}{(1+x)^{2}}\right) f_{3}\left(\frac{1}{1+x}\right)\right]-\frac{2}{3} \frac{C}{\sqrt{x}},
$$

which slowly approaches unity as $x$ increases:

$$
w(x) \rightarrow 1-\frac{2}{3} \frac{C}{\sqrt{x}}+\frac{1}{4 x}
$$

For very large $x>>1, w(x) \rightarrow 1$ and expression (8.22) is just the energy one gets using only the direct field of the magnet bore. For $x>.2$, i.e. $\ell>.63 R$, which covers most cases of practical interest,

$$
w(x) \approx \frac{1}{1+\frac{.63}{\sqrt{x}}}
$$

with error less that $1 \%$, so we have in general for the thin layer lens

$$
W \approx \frac{\pi R^{2} \ell\left(\mu_{0} S\right)^{2}}{2 \mu_{0}} \frac{1}{1+.89 R / \ell}
$$

It is surprising that the value of $W$ for the finite-length lens is smaller than the approximate value found using only the direct field.

Suppose the current layer is made of $n$ turns of wire, each carrying current I . Then $S=n \mathrm{I} / \ell-$ see section 2.3 for a discussion of effective length $\ell$, and the magnet's inductance is

$$
L=\frac{2 W}{\mathrm{I}^{2}} \approx \frac{\mu_{0} n^{2} \pi R^{2}}{\ell+.89 R}
$$

\subsection{Current ring}

Some solenoid systems are made out of a set of current rings. We derived the onaxis field of a ring in section 4-1, and here we derive its field for all $(r, z)$. The ring itself is taken to have infinitesimal thickness, so the magnetic field strongly diverges at the wire. Simple exact and approximate results are found in this somewhat unphysical limit. 
Formulas for a ring of finite thickness with rectangular cross-section can be written down using results from section 9 .

As in section 4-1 we assume current density

$$
J_{\theta}=I \delta(r-R) \delta(z),
$$

and the on-axis field is

$$
B_{0}^{\text {ring }}(z)=\frac{\mu_{0} I R^{2}}{2{\sqrt{z^{2}+R^{2}}}^{3}}
$$

The two leading terms of the near-axis expansions are

$$
\begin{aligned}
& B_{z} \approx B_{0}-B_{0}{ }^{\prime \prime} \frac{r^{2}}{4}=\frac{\mu_{0} I R^{2}}{2}\left[\frac{1}{{\sqrt{z^{2}+R^{2}}}^{3}}-\frac{\left(12 z^{2}-3 R^{2}\right)}{\sqrt{z^{2}+R^{2}}} \frac{r^{2}}{4}\right], \\
& B_{r} \approx-B_{0}{ }^{\prime} \frac{r}{2}+B_{0}^{\prime \prime \prime} \frac{r^{3}}{16}=\frac{\mu_{0} I R^{2}}{2}\left[\frac{3 z}{\sqrt{z^{2}+R^{2}}}{ }^{5} \frac{r}{2}+\frac{45 R^{2} z-60 z^{3}}{\sqrt{z^{2}+R^{2}}} \frac{r^{3}}{16}\right] \text {. }
\end{aligned}
$$

For general $(r, z)$ eqns. (6.26. 6.27, and 6.28) yield

$$
\begin{aligned}
& B_{z}=\frac{\mu_{0} I R^{2}}{2} \frac{f_{4}(b)-\frac{r}{R} f_{3}(b)}{{\sqrt{z^{2}+r^{2}+R^{2}}}^{3}}, \\
& B_{r}=\frac{\mu_{0} I R z}{2} \frac{f_{3}(b)}{{\sqrt{z^{2}+r^{2}+R^{2}}}^{3}}, \\
& A_{\theta}=\frac{\mu_{0} I R}{2} \frac{f_{1}(b)}{\sqrt{z^{2}+r^{2}+R^{2}}},
\end{aligned}
$$

with $b=2 r R /\left(z^{2}+r^{2}+R^{2}\right)$. However eqn. (8-33) is much more easily derived from eqn. (6-14) using $B_{r}=-\partial A_{\theta} / \partial z$.

Accurate approximate formulas for $B_{z}, B_{r}$, and $\mathrm{A}_{\theta}$ are found by substituting the approximate formulas for $f_{i}(b)$ from section 7-4 into eqns (8.32, 8.33, and 8.34); here we display only the expression for $A_{\theta}$ : 


$$
\begin{aligned}
& A_{\theta} \approx \frac{\mu_{0} I R}{2} \frac{1}{\sqrt{z^{2}+r^{2}+R^{2}}} \frac{C}{4 b}\left\{\left[D-\frac{2+D}{16}\left(1-b^{2}\right)-\log \left(1-\mathrm{b}^{2}\right)+\frac{1}{16}\left(1-\mathrm{b}^{2}\right) \log \left(1-\mathrm{b}^{2}\right)\right]\right. \\
& \left.+\left[\left(\frac{2}{16}-\frac{15 D}{16}\right)+\left(\frac{1}{C}-\frac{13}{16}-\frac{31 D}{16}\right) b^{2}+\left(\frac{79}{32 C}-\frac{73}{32}-\frac{47 D}{16}\right) b^{4}\right]\left(1-b^{2}\right)^{2}\right\},
\end{aligned}
$$

which has a maximum relative error of $.029 \%$ at $b=.73$.

The singular limit of the ring current field close to the wire is found by expanding eqn. (8.35) with

$$
\begin{aligned}
& s \equiv r-R<R, \quad z<<R: \\
& A_{\theta} \rightarrow \frac{\mu_{0} I}{4 \pi}\left[D+\frac{s}{R}\left(1-\frac{D}{2}\right)-\log \left(\frac{\mathrm{z}^{2}+s^{2}}{R^{2}}\right)+\frac{s}{2 R} \log \left(\frac{\mathrm{z}^{2}+s^{2}}{R^{2}}\right)\right], \\
& B_{r}=-\frac{\partial A_{\theta}}{\partial z} \rightarrow \frac{\mu_{0} I}{2 \pi}\left(\frac{z}{z^{2}+s^{2}}\right)\left(1-\frac{s}{2 R}\right), \\
& B_{z}=\frac{1}{r} \frac{\partial}{\partial r} r A_{\theta} \rightarrow \frac{\mu_{0} I}{4 \pi R}\left[\left(1+\frac{D}{2}\right)-\frac{1}{2} \log \left(\frac{\mathrm{z}^{2}+s^{2}}{R^{2}}\right)+\frac{s^{2}}{z^{2}+s^{2}}-\frac{2 R s}{z^{2}+s^{2}}\right] .
\end{aligned}
$$

The relative error in eqns. $(8.36,8.37$, and 8.38$)$ is less than $1.2 \%$ for $\sqrt{s^{2}+z^{2}}<R / 10$. Very near the wire $\vec{B}$ diverges as

$$
\vec{B} \rightarrow \frac{\mu_{0} I}{2 \pi} \frac{z \hat{e}_{r}-s \hat{e}_{z}}{z^{2}+s^{2}}
$$

as expected, plus an additional divergent logarithmic term for $B_{z}$.

\subsection{Approximation of fields and potentials using elementary functions}

In section 8.2 we derived expressions for the fields and potentials $\left(B_{r}, B_{z}, A_{\theta}, \varphi\right)$ of the semi-infinite thin current layer, $J_{\theta}=S \delta(r-R) \Theta(z)$. Those exact global results involve only integrations over $\theta$, but it would aid computations if the integrals could be evaluated using only elementary functions. That appears to be impossible, but it is found that the four fields/potentials can be approximated with fair accuracy using functions that are no more complicated than the arctangent. While such approximations are not unique, they are strongly constrained if we accept these conditions:

accurate for all $(r, z)$, e.g. less than $1 \%$ error;

simple-only one or two lines of symbols;

only use elementary functions; 
correct limits as $r \rightarrow 0$ and $\infty$;

continuous (except for $B_{z}$ ) at the current layer;

correct lowest order aberration.

This is all possible except for an obvious tradeoff between accuracy and simplicity, and the interested reader can easily improve on the accuracy of the expressions given below.

\section{Approximation of $B_{r}$}

This is the most straightforward case. We start with eqn. (8.13):

$$
B_{r}=\frac{\mu_{0} S R}{2} \frac{f_{1}(b)}{\sqrt{z^{2}+r^{2}+R^{2}}},
$$

with $b(r, z)=2 r R /\left(z^{2}+r^{2}+R^{2}\right)$. It is only necessary to insert a sufficiently accurate, but elementary, expression for $f_{1}(b)$. Eqn. (7.10) is suitable:

$$
B_{r} \approx \frac{\mu_{0} S R}{2 \sqrt{z^{2}+r^{2}+R^{2}}} \frac{C}{4 b}\left[\left(\frac{1}{C}-1\right) b^{2}+\left(1+D-\frac{1}{C}\right) b^{4}-\log \left(1-b^{2}\right)\right],
$$

with $C=2 \sqrt{2} / \pi$ and $D=6 \log (2)-4$. The maximum relative error of .726\% occurs for $b(r, z)=.744$, which is near the current layer end.

\section{Approximation of $B_{z}$}

The trick here is to write $B_{z}$ as the sum of direct and residual parts:

$$
B_{z}=\mu_{0} S \Theta(r-R) \Theta(z)+B_{z}^{\text {residual }}
$$

and then approximate $B_{z}^{\text {residual }}$. For $z=0$ and $r>R, B_{z}^{\text {residual }}$ vanishes, and as $z \rightarrow 0$ it approaches $\pm \mu_{0} S / 2$ for $r<R$. A simple expression, which can almost be written down from an inspection of the various limits, is

$$
B_{z}^{\text {residual }} \approx \frac{\mu_{0} S}{2} \frac{z}{|z|}\left(\sqrt{\frac{z^{2}}{R^{2}}+1}-\frac{|z|}{R}\right) \frac{1}{2}\left[\frac{r+R}{\sqrt{z^{2}+(r+R)^{2}}}-\frac{(r-R)}{\sqrt{z^{2}+(r-R)^{2}}}\right] .
$$

This formula meets the conditions given above except that it is inaccurate close to the current layer end (maximum error of $.14 \mu_{0} S / 2$ ) and does not have the correct lowest order aberration, but it does include all of the important qualitative features of the exact $B_{z}^{\text {residual }}$. It is derived below along with several improved versions. 
We start by observing that since

$$
\frac{\partial B_{z}^{\text {residual }}}{\partial \mathrm{r}}=\frac{\partial B_{r}}{\partial z}
$$

and $B_{z}^{\text {residual }} \rightarrow 0$ as $r \rightarrow \infty$, we have from eqn. (8.4b):

$$
\begin{aligned}
B_{z}^{\text {residual }} & =\int_{\infty}^{r} d r^{\prime} \frac{\partial B_{r}\left(r^{\prime}, z\right)}{\partial z}=-\frac{\mu_{0} S R z}{2} \int_{0}^{2 \pi} \frac{d \theta}{2 \pi} \cos (\theta) \int_{\infty}^{r} d r^{\prime} \frac{1}{{\sqrt{z^{2}+r^{\prime 2}+R^{2}-2 r^{\prime} R \cos (\theta)}}^{3}} \\
& =-\frac{\mu_{0} S R z}{2} \int_{0}^{2 \pi} \frac{d \theta}{2 \pi} \frac{\cos (\theta)}{z^{2}+R^{2} \sin ^{2}(\theta)} \frac{r-R \cos (\theta)}{\sqrt{z^{2}+r^{2}+R^{2}-2 r R \cos (\theta)}}
\end{aligned}
$$

Here we used eqn. (3.10a) after changing the variable of integration from $r^{\prime}$ to $r^{\prime}-R \cos (\theta)$. In order to approximate the expression (8.45) it is convenient to write it in the compact form:

$$
\begin{aligned}
& B_{z}^{\text {residual }}=\frac{\partial \psi}{\partial r}, \\
& \psi(r, z)=\frac{\mu_{0} S}{2} \frac{z}{|z|} \sqrt{z^{2}+r^{2}+R^{2}} J\left(\frac{z}{R}, b\right), \\
& J\left(\frac{z}{R}, b\right)=-\frac{|z|}{R} \int_{0}^{2 \pi} \frac{d \theta}{2 \pi} \frac{\cos (\theta)}{\frac{\mathrm{z}^{2}}{R^{2}}+\sin ^{2}(\theta)} \sqrt{1-b \cos (\theta)} .
\end{aligned}
$$

We now expand $J$ in (odd) powers of $b$ :

$$
J\left(\frac{z}{R}, b\right)=j_{1}\left(\frac{z}{R}\right) \alpha_{1} b+j_{3}\left(\frac{z}{R}\right) \alpha_{3} b^{3}+j_{5}\left(\frac{z}{R}\right) \alpha_{5} b^{5}+\ldots,
$$

with the coefficients $\alpha_{i}$ from the Taylor series expansion of $-\sqrt{1-b \cos (\theta)}$ :

$$
\alpha_{1}=\frac{1}{2}, \alpha_{3}=\frac{1}{16}, \alpha_{5}=\frac{7}{256}, \alpha_{7}=\frac{33}{2048}, \ldots
$$

The functions $j_{n}(z / R)$ are defined for odd $n$ as

$$
j_{n}(x)=|x| \int_{0}^{2 \pi} \frac{d \theta}{2 \pi} \frac{\cos ^{\mathrm{n}+1}(\theta)}{x^{2}+\sin ^{2}(\theta)}
$$




$$
\begin{aligned}
& j_{1}(x)=\sqrt{x^{2}+1}-|\mathrm{x}|, \\
& j_{3}(x)={\sqrt{x^{2}+1}}^{3}-|x|^{3}-\frac{3}{2}|x|, \\
& j_{5}(x)={\sqrt{x^{2}+1}}^{5}-|x|^{5}-\frac{5}{2}|x|^{3}-\frac{15}{8}|x|, \\
& j_{n+2}(x)=\left(1+x^{2}\right) j_{n}(x)-\frac{(n+1) !}{2^{n+1}\left(\frac{n+1}{2} !\right)^{2}}|x| .
\end{aligned}
$$

The series (8.49) converges slowly, but this situation is greatly improved by making use of the expansion

$$
\frac{\sqrt{1+b}-\sqrt{1-b}}{2}=\alpha_{1} b+\alpha_{3} b^{3}+\alpha_{5} b^{5} \ldots
$$

Thus we can write

$$
\begin{gathered}
J(x, b)=j_{1}(x) \frac{1}{2}(\sqrt{1+b}-\sqrt{1-b})+\left(j_{3}(x)-j_{1}(x)\right) \alpha_{3} b^{3} \\
+\left(j_{5}(x)-j_{1}(x)\right) \alpha_{5} b^{5}+\ldots
\end{gathered}
$$

The leading term $\left(\sim j_{1}(x)\right)$ gives a fair approximation of $J(x, b)$; additional terms such as $\left(j_{3}-j_{1}\right) \alpha_{3} b^{3}$ are small, but their sum converges slowly for $x \approx .1$ and $b \rightarrow 1$. Keeping only the leading term we have

$$
\begin{aligned}
& \psi \approx \frac{\mu_{0} S}{2} \frac{z}{|z|} \sqrt{z^{2}+r^{2}+R^{2}} j_{1}\left(\frac{z}{R}\right) \frac{1}{2}(\sqrt{1+b}-\sqrt{1-b}), \\
& =\frac{\mu_{0} S}{2} \frac{z}{|z|}\left(\sqrt{\frac{z^{2}}{R^{2}}+1}-\frac{|z|}{R}\right) \frac{1}{2}\left(\sqrt{z^{2}+(r+R)^{2}}-\sqrt{z+(r-R)^{2}}\right) .
\end{aligned}
$$

Applying $B_{z}^{\text {residual }}=\partial \psi / \partial r$ we get the advertised result, eqn (8.43).

There are several tricks that improve accuracy (with increased complexity); here we only use the simplest of these, which exploits the decreasing difference between the $j_{n}(x)$ as $n$ increases. That is to write eqn. (8.49) as 


$$
J(x, b)=j_{1}(x) \frac{b}{2}+j_{3}(x)\left(\frac{\sqrt{1+b}-\sqrt{1-b}}{2}-\frac{b}{2}\right)+\left(j_{5}(x)-j_{3}(x)\right) \alpha_{5} b^{5}+\ldots,
$$

and drop terms $\sim\left(j_{n}-j_{3}\right)$. We get

$$
\begin{aligned}
& \left.\psi \approx \frac{\mu_{0} S}{2} \frac{z}{|z|} \mid\left\{j_{1}\left(\frac{z}{R}\right)-j_{3}\left(\frac{z}{R}\right)\right] \frac{r R}{\sqrt{z^{2}+r^{2}+R^{2}}}+j_{3}\left(\frac{z}{R}\right)\left[\frac{\sqrt{z^{2}+(r+R)^{2}}-\sqrt{z^{2}+(r-R)^{2}}}{2}\right]\right\} \\
& B_{z}^{\text {residual }}=\frac{\partial \psi}{\partial r} \approx \frac{\mu_{0} S}{2} \frac{z}{|z|}\left[\left(\sqrt{\frac{z^{2}}{R^{2}}+1}-\left|\frac{z}{R}\right|-{\sqrt{\frac{z^{2}}{R^{2}}+1}}^{3}+\left|\frac{z}{R}\right|^{3}+\frac{3}{2} \mid \frac{z}{R}\right)\left(\frac{R}{\sqrt{z^{2}+r^{2}+R^{2}}}-\frac{r^{2} R}{{\sqrt{z^{2}+r^{2}+R^{2}}}^{3}}\right)\right. \\
& \left.+\left(\sqrt{\frac{z^{2}}{R^{2}}+1}-\left.|| \frac{z}{R}\right|^{3}-\frac{3}{2} \mid \frac{z}{R}\right) \mid \frac{1}{2}\left(\frac{(r+R)}{\sqrt{z^{2}+(r+R)^{2}}}-\frac{(r-R)}{\sqrt{z^{2}+(r-R)^{2}}}\right)\right] .
\end{aligned}
$$

Accuracy is now excellent everywhere except very close to the current layer end, but maximum error is still about $.14 \mu_{0} S / 2$ in that zone. The lowest order aberration is recovered as $r \rightarrow 0$.

\section{Approximation of $A_{\theta}$}

A very accurate global approximation of $A_{\theta}$ can be written down, but it is too complicated to be worth presenting here. However, a general approach that gives simple but not very accurate formulas, as well as more complicated but reasonably accurate ones, is as follows. We write

$$
A_{\theta}=-\int_{\infty}^{z} d z^{\prime} B_{r}\left(r, z^{\prime}\right)=\int_{z}^{\infty} d z^{\prime} \frac{\mu_{0} S R}{2} \frac{f_{1}\left(b^{\prime}\right)}{\sqrt{z^{\prime 2}+r^{2}+R^{2}}},
$$

where $b^{\prime}=2 r R /\left(z^{\prime 2}+r^{2}+R^{2}\right)$. Making the change of variable

$$
y=\frac{z^{\prime}}{\sqrt{z^{\prime 2}+r^{2}+R^{2}}}, \quad b^{\prime}=\frac{2 r R\left(1-y^{2}\right)}{r^{2}+R^{2}},
$$

we get

$$
A_{\theta}=\frac{\mu_{0} S R^{2} r}{4\left(r^{2}+R^{2}\right)} \underset{\frac{z}{\sqrt{z^{2}+r^{2}+R^{2}}}}{\int^{1} d y}\left[\frac{f_{1}\left(b^{\prime}\right)}{b^{\prime} / 4}\right] .
$$


The idea is to approximate $f_{1}\left(b^{\prime}\right) /\left(b^{\prime} / 4\right)$ using functions of $b^{\prime}(y)$ that result in simple integrals in eqn. (8.61). The expansion (7.2b),

$$
\frac{f_{1}\left(b^{\prime}\right)}{b^{\prime} / 4}=1+\frac{15}{32} b^{\prime 2}+\frac{315}{1024} b^{\prime 4}+\ldots
$$

does this, but convergence is poor for $b^{\prime} \geq .5$. This means that a truncation of the expansion will give inaccurate results near the current layer. To do a good job requires that a term $\sim-C \log \left(1-\mathrm{b}^{\prime 2}\right)$ be included in the approximation of $f_{1}\left(b^{\prime}\right) /\left(b^{\prime} / 4\right)$, but that leads to a more complicated final result. Instead of the power series, eqn. (8.62), we use

$$
\frac{f_{1}\left(b^{\prime}\right)}{b^{\prime} / 4} \approx 1+\frac{15}{32} b^{\prime 2}-(.27) b^{\prime 4}-C\left[\log \left(1-b^{\prime 2}\right)+b^{\prime 2}\right],
$$

which has a maximum relative error of about $3 \%$ near $b^{\prime}=.994$. The integration over $y$ is elementary, but produces many terms involving both $r$ and $z$. It may be written compactly by defining the functions

$$
p(r)=\frac{2 r R}{r^{2}+R^{2}}, \quad q(r, z)=\frac{z}{\sqrt{z^{2}+r^{2}+R^{2}}} .
$$

Then we finally get

$$
\begin{aligned}
A_{\theta} & \approx\left(\frac{\mu_{0} S R}{8}\right) p\left\{(1-q)+\left(\frac{15}{32}-C\right) p^{2}\left(\frac{8}{15}-q+\frac{2 q^{3}}{3}-\frac{q^{5}}{5}\right)\right. \\
& -(.27) p^{4}\left(\frac{128}{315}-q+\frac{4 q^{3}}{3}-\frac{6 q^{5}}{5}+\frac{4 q^{7}}{7}-\frac{q^{9}}{9}\right) \\
& -C\left[-q \log \left(1-\mathrm{p}^{2}\left(1-q^{2}\right)^{2}\right)-4(1-q)\right. \\
& +2 \sqrt{\frac{1-p}{p}}\left(\tan ^{-1}\left(\sqrt{\frac{p}{1-p}}\right)-\tan ^{-1}\left(q \sqrt{\frac{p}{1-p}}\right)\right) \\
& \left.\left.+\sqrt{\frac{1+p}{p}} \log \left(\frac{1+\sqrt{\frac{\mathrm{p}}{1+\mathrm{p}}}}{1-\sqrt{\frac{p}{1+p}}} \cdot \frac{1-q \sqrt{\frac{\mathrm{p}}{1+\mathrm{p}}}}{1+q \sqrt{\frac{p}{1+p}}}\right)\right]\right)
\end{aligned}
$$

This approximate potential has maximum error of about $1.5 \%$. It has the correct limits as $r \rightarrow 0$ and $\infty$ and the correct lowest order aberration. It should be of some value for 
plotting flux surfaces $\left(r A_{\theta}=\right.$ constant $)$, but deriving $B_{z}=\frac{1}{r} \frac{\partial}{\partial r} r A_{\theta}$ leads to a very complicated formula.

\section{Approximate Global Potential $\varphi$}

There seems to be little point in approximating $\varphi$ everywhere, and the formulas are always complicated for even moderate accuracy, so none are written down here. A good way to generate such formulas is to plug known approximations for $B_{r}, B_{z}^{\text {residual }}$, and $f_{0}(b)$ - see section 7 , into

$$
\varphi=z B_{z}^{\text {residual }}+(r-R b) B_{r}-\frac{\mu_{0} S R^{2}}{2} \frac{f_{0}(b)}{\sqrt{z^{2}+r^{2}+R^{2}}} .
$$

The derivation of eqn. (8.66) is too lengthy to include here, but it is similar to the derivation of eqn. (8.45).

\section{Thick Current Layer}

\subsection{Uniform thick layer}

While the thin current layer formulas given in sections 4 and 8 are sufficient for most beam dynamics applications, thick layer results are needed for precise near-axis fields, external fields, field in the wire, and material stress. We first treat the case of radially uniform current density:

$$
J_{\theta}=\frac{S(z)}{R_{2}-R_{1}} \quad\left(R_{1}<r<R_{2}\right) .
$$

As in section 4, it is usually sufficient to derive the field for a uniform, semi-infinite, thick current layer, $S(z)=S \Theta(z)$, and any system of lenses may be built up by translations and super-position.

Recall from section 8 the semi-infinite thin layer field $\left[J_{\theta}=S \Theta(z) \delta(r-R)\right]$ :

$$
\begin{aligned}
& B_{z}^{\text {thin }}=\frac{\mu_{0} S}{2} \int_{0}^{2 \pi} \frac{d \theta}{2 \pi} \frac{R^{2}-R r \cos (\theta)}{R^{2}+r^{2}-2 R r \cos (\theta)}\left[1-\frac{z}{\sqrt{z^{2}+R^{2}+r^{2}-2 R r \cos (\theta)}}\right], \\
& B_{r}^{\text {thin }}=\frac{\mu_{0} S}{2} \int_{0}^{2 \pi} \frac{d \theta}{2 \pi} \frac{R \cos (\theta)}{\sqrt{z^{2}+R^{2}+r^{2}-2 R r \cos (\theta)}} .
\end{aligned}
$$


For the thick, uniform, semi-infinite layer we simply compute a radial superposition of these thin layer fields:

$$
\left(B_{z}, B_{r}\right)^{\text {thick }}=\int_{R_{1}}^{R_{2}} \frac{d R}{R_{2}-R_{1}}\left(B_{z}, B_{r}\right)^{\text {thin }}
$$

The on-axis field of the semi-infinite thick layer is

$$
\begin{aligned}
B_{0}^{\text {thick }}(z)=\int_{R_{1}}^{R_{2}} & \frac{d R}{R_{2}-R_{1}} \frac{\mu_{0} S}{2}\left(1-\frac{z}{\sqrt{z^{2}+R^{2}}}\right) \\
& =\frac{\mu_{0} S}{2}\left[1-\frac{z}{R_{2}-R_{1}} \log \left(\frac{R_{2}+\sqrt{z^{2}+R_{2}^{2}}}{R_{1}+\sqrt{z^{2}+R_{1}^{2}}}\right)\right] .
\end{aligned}
$$

For a thick-layer lens of length $\ell$ and centered at $z=0$, the on-axis field is therefore

$$
\begin{aligned}
B_{0}^{\mathrm{Lens}}(z)=\frac{\mu_{0} S}{2\left(R_{2}-R_{1}\right)}\left\{\left(\frac{\ell}{2}+z\right) \log \left[\frac{R_{2}+\sqrt{\left(\frac{\ell}{2}+z\right)^{2}+R_{2}^{2}}}{R_{1}+\sqrt{\left(\frac{\ell}{2}+z\right)^{2}+R_{1}^{2}}}\right]\right. \\
+\left(\frac{\ell}{2}-z\right) \log \left[\frac{\left.R_{2}+\sqrt{\left(\frac{\ell}{2}-z\right)^{2}+R_{2}^{2}}\right]}{\left.R_{1}+\sqrt{\left(\frac{\ell}{2}-z\right)^{2}+R_{1}^{2}}\right]} .\right.
\end{aligned}
$$

Near-axis values of $B_{z}$ and $B_{r}$ can then be obtained using these results and the radial expansions of section 3 . For the field at the magnet center $(z=0)$, eqn. (9.5) reduces to

$$
B_{0}^{\text {Lens }}(0)=\frac{\mu_{0} S \ell}{2\left(R_{2}-R_{1}\right)} \log \left(\frac{R_{2}+\sqrt{\ell^{2} / 4+R_{2}^{2}}}{R_{1}+\sqrt{\ell^{2} / 4+R_{1}^{2}}}\right) .
$$

The semi-infinite, thick-layer formulas for $B_{z}$ and $B_{r}$ at general $(r, z)$ can also be written in terms of integrations over $\theta$. The integrals over $R$ in eqn. (9.3) can actually be evaluated in terms of elementary functions, although the results are somewhat messy. But the speed of computation is greatly increased over the direct application of eqn. (9.3), especially inside the current layer. First we define

$$
u(r, \theta)=R_{1}-r \cos (\theta), v(r, \theta)=R_{2}-r \cos (\theta)
$$


Then, using the integrals given at the end of section 3 and omitting some intermediate steps, we get from eqns. $(9.2,9.3)$ :

$$
\begin{aligned}
& B_{r}^{\text {thick }}=\frac{\mu_{0} S}{2\left(R_{2}-R_{1}\right)} \int_{0}^{2 \pi} \frac{d \theta}{2 \pi} \cos (\theta)\left\{\sqrt{v^{2}+z^{2}+r^{2} \sin ^{2}(\theta)}\right. \\
& \left.-\sqrt{u^{2}+z^{2}+r^{2} \sin ^{2}(\theta)}+r \cos (\theta) \log \left[\frac{v+\sqrt{v^{2}+z^{2}+r^{2} \sin ^{2}(\theta)}}{u+\sqrt{u^{2}+z^{2}+r^{2} \sin ^{2}(\theta)}}\right]\right\}, \\
& B_{z}^{\text {thick }}=\frac{\mu_{0} S(r)}{2}-\frac{\mu_{0} S z}{2\left(R_{2}-R_{1}\right)} \int_{0}^{2 \pi} \frac{d \theta}{2 \pi}\left\{\log \left[\frac{v+\sqrt{v^{2}+z^{2}+r^{2} \sin ^{2}(\theta)}}{u+\sqrt{u^{2}+z^{2}+r^{2} \sin ^{2}(\theta)}}\right]\right. \\
& -\left(\frac{r \cos (\theta)}{z}\right)\left[\tanh ^{-1}\left(\frac{\sqrt{v^{2}+z^{2}+r^{2} \sin ^{2}(\theta)}}{z}\right)-\tanh ^{-1}\left(\frac{\sqrt{u^{2}+z^{2}+r^{2} \sin ^{2}(\theta)}}{z}\right)\right] \\
& \left.-\left(\frac{r \sin (\theta)}{z}\right)\left[\tan ^{-1}\left(\frac{z}{r \sin (\theta)} \frac{v}{\sqrt{v^{2}+z^{2}+r^{2} \sin ^{2}(\theta)}}\right)-\tan ^{-1}\left(\frac{z}{r \sin (\theta)} \frac{u}{\sqrt{u^{2}+z^{2}+r^{2} \sin ^{2}(\theta)}}\right)\right]\right\} \text {. }
\end{aligned}
$$

Here we have used the cumulative current distribution,

$$
S(r)= \begin{cases}S & 0<r<R_{1}, \\ S \frac{R_{2}-r}{R_{2}-R_{1}} & R_{1}<r<R_{2}, \\ 0 & R_{2}<r .\end{cases}
$$

\subsection{Approximation by a thin layer}

It was asserted in section 4 that, with only minor error, the on-axis field of a thin current layer could replace the on-axis field of a uniform thick layer. There are many ways to select the three parameters $(S, R, \ell)$ of the equivalent thin layer. Here we use the same $\mathrm{S}$ and $\ell$ as the thick layer and set $\mathrm{R}$ to give the correct net magnetic dipole moment:

$$
\mathrm{R}^{2}=\int_{0}^{\infty} \frac{d r J_{\theta} r^{2}}{S}=\int_{R_{1}}^{R_{2}} d r \frac{1}{R_{2}-R_{1}} r^{2}=\frac{1}{3}\left(R_{2}^{2}+R_{2} R_{1}+R_{1}^{2}\right) .
$$

Defining a thickness parameter 


$$
\Delta \equiv\left(R_{2}-R_{1}\right) / R,
$$

we have from eqn. (9.11),

$$
R_{1}=R\left(\sqrt{1-\frac{\Delta^{2}}{12}}-\frac{\Delta}{2}\right), \quad R_{2}=R\left(\sqrt{1-\frac{\Delta^{2}}{12}}+\frac{\Delta}{2}\right) .
$$

We require that $\Delta<\sqrt{3}$ to prevent $R_{1}<0$. A somewhat different formula for $R$ is obtained if we equate the focal lengths of the magnets $\left(f^{-1} \sim \int d z B_{0}^{2}(z)\right)$. The following table gives the maximum ratio of on-axis fields for the thin layer, eqn. (4.6), relative to the equivalent thick layer, eqn. (9.5), for various values of $\Delta$ and $\ell / R$. Except for very small $\ell / R$ this maximum occurs outside the magnet at $z \approx \pm(\ell / 2+.8 R)$. Around $z=0$ the near axis expansion is unreliable as $r \rightarrow R_{1}$.

\begin{tabular}{|l|l|l|l|l|}
\hline & $B_{0}^{\text {thin }} / B_{0}^{\text {thick }}$ & & & \\
$\ell / R$ & $\Delta=.1$ & $\Delta=.2$ & $\Delta=.5$ & $\Delta=1.0$ \\
\hline 1 & 1.00094 & 1.0038 & 1.024 & 1.101 \\
\hline 2 & 1.00088 & 1.0035 & 1.023 & 1.094 \\
\hline 4 & 1.00082 & 1.0033 & 1.021 & 1.087 \\
\hline 8 & 1.00079 & 1.0032 & 1.020 & 1.084 \\
\hline 16 & 1.00078 & 1.0031 & 1.020 & 1.083 \\
\hline
\end{tabular}

\subsection{General thick layer - Bessel transform solution}

We treat a current layer with fixed ends and general current density $J_{\theta}(r)=-d S(r) / d r$ between the ends. The Green function solutions of section 6 can be used for this, as can weighted averages of eqns. (9.2a,b). However, different and possibly useful expressions can be derived using the Bessel transform.

First, the semi-infinite layer, $J_{\theta}=J_{\theta}(r) \Theta(z)$, has $B_{z}(r, z \rightarrow-\infty)=\mu_{0} S(r)$, and by symmetry

$$
B_{z}(r, z=0)=\frac{\mu_{0} S(r)}{2}
$$

For $z>0$,

$$
B_{z} \sim J_{0}(k r) e^{-k z}
$$


with $k>0$, is a valid solution of $\nabla^{2} B_{z}=0$, except for the boundary condition (9.14). The correct solution for $z \geq 0$ is given by the Bessel transform:

$$
\begin{aligned}
& B_{z}(r, z \geq 0)=\frac{\mu_{0}}{2} \int_{0}^{\infty} d k \quad k J_{0}(k r) e^{-k z} T(k), \\
& T(k)=\int_{0}^{\infty} d r r J_{0}(k r) S(r) .
\end{aligned}
$$

A calculation similar to the above gives

$$
B_{z}(r, z \leq 0)=\mu_{0} S(r)-\frac{\mu_{0}}{2} \int_{0}^{\infty} d k k J_{0}(k r) e^{+k z} T(k) .
$$

Combining these results we have for the entire semi-infinite layer:

$$
B_{z}=\mu_{0} S(r) \Theta(z)+\frac{z}{|z|} \frac{\mu_{0}}{2} \int_{0}^{\infty} d k \quad k J_{0}(k r) e^{-k|z|} T(k)
$$

The magnetic field can also be derived from the global scalar potential - see section 5:

$$
B_{z}(r, z)=\mu_{0} S(r) \Theta(z)+\partial \varphi / \partial z
$$

Equation (9.19) then gives for $\varphi$ and $B_{r}$ :

$$
\begin{aligned}
& \varphi(r, z)=-\frac{\mu_{0}}{2} \int_{0}^{\infty} d k J_{0}(k r) e^{-k|z|} T(k), \\
& B_{r}(r, z)=\frac{\partial \varphi}{\partial r}=\frac{\mu_{0}}{2} \int_{0}^{\infty} d k \quad k J_{1}(k r) e^{-k|z|} T(k) .
\end{aligned}
$$

Using superposition, a lens of length $\ell$, centered at $z=0$, has fields

$$
\begin{aligned}
& B_{z}(r, z)=\mu_{0} S(r)[\Theta(z-\ell / 2)-\Theta(z+\ell / 2)] \\
& +\frac{\mu_{0}}{2} \int_{0}^{\infty} d k \quad k J_{0}(k r) T(k)\left[\frac{(z-\ell / 2)}{|z-\ell / 2|} e^{-k|z-\ell / 2|}-\frac{(z+\ell / 2)}{|z+\ell / 2|} e^{-k|z+\ell / 2|}\right], \\
& B_{r}(r, z)=\frac{\mu_{0}}{2} \int_{0}^{\infty} d k k J_{1}(k r) T(k)\left[e^{-k|z-\ell / 2|}-e^{-k|z+\ell / 2|}\right] .
\end{aligned}
$$

It remains to evaluate $T(k)$; we can get analytical results for several cases. First we integrate by parts in eqn. (9.17) using $J_{\theta}=-d S(r) / d r$ : 


$$
T(k)=\int_{0}^{\infty} d r S(r) \frac{\partial}{\partial r} \frac{r J_{1}(k r)}{k}=\int_{0}^{\infty} d r J_{\theta}(r) \frac{r J_{1}(k r)}{k}
$$

Case 1 (thin layer):

$$
\begin{aligned}
& J_{\theta}(r)=S \delta(r-R), \\
& T(k)=\frac{R S J_{1}(k R)}{k}
\end{aligned}
$$

Case $2 \quad\left(J_{\theta}\right.$ decreasing as $\left.1 / r\right)$ :

$$
\begin{aligned}
& J_{\theta}(r)=\frac{S}{r \log \left(R_{2} / R_{1}\right)} \quad R_{1}<r<R_{2}, \\
& T(k)=\frac{S}{k^{2} \log \left(R_{2} / R_{1}\right)}\left[J_{0}\left(k R_{1}\right)-J_{0}\left(k R_{2}\right)\right] .
\end{aligned}
$$

Case 3 (uniform thick layer):

$$
\begin{aligned}
& J_{\theta}=\frac{S}{R_{2}-R_{1}} \quad R_{1}<r<R_{2}, \\
& T(k)=\frac{S}{k^{3}\left(R_{2}-R_{1}\right)} \int_{k R_{1}}^{k R_{2}} d y y J_{1}(y) .
\end{aligned}
$$

The integration over $y$, although not elementary, can be expressed as a hypergeometric function:

$$
\int_{0}^{x} d y y J_{1}(y)=\frac{x^{3}}{6} \quad{ }_{1} F_{2}\left(\frac{3}{2} ; 2, \frac{5}{2} ;-x^{2} / 4\right) .
$$

In Mathematica ${ }^{\circledR}$ notation:

$$
\begin{aligned}
& { }_{1} F_{2}(a ; b, c ; z)=\text { Hypergeometic PFQ }[\{\mathrm{a}\},\{b, c\}, z] \\
& =1+\frac{a}{b c} \frac{z}{1 !}+\frac{a(a+1)}{b(b+1) c(c+1)} \frac{z^{2}}{2 !}+\ldots .
\end{aligned}
$$

Equation (9.31) becomes 


$$
T(k)=\frac{S}{6\left(R_{2}-R_{1}\right)}\left[\left(R_{2}^{3}\right)_{1} F_{2}\left(\frac{3}{2} ; 2, \frac{5}{2} ;-\frac{k^{2} R_{2}^{2}}{4}\right)-R_{1}^{3}{ }_{1} F_{2}\left(\frac{3}{2} ; 2, \frac{5}{2} ; \frac{-k^{2} R_{1}^{2}}{4}\right)\right] .
$$

\subsection{Pancake current layer}

Some applications may require a current layer that is radially thick but longitudinally thin, like a pancake with a hole in the middle or a compact disc. The radially uniform current density is approximated by

$$
J_{\theta}^{\mathrm{PC}}=\frac{I \delta(z)}{R_{2}-R_{1}} \quad R_{1}<r<R_{2} .
$$

The fields from such a layer are easily calculated using the Green function formalism of section (6) and we only give the results here:

$$
\begin{aligned}
& B_{0}^{\mathrm{PC}}(z)=\frac{\mu_{0} I}{2\left(R_{2}-R_{1}\right)}\left[\log \left(\frac{\mathrm{R}_{2}+\sqrt{z^{2}+R_{2}^{2}}}{R_{1}+\sqrt{z^{2}+R_{1}^{2}}}\right)-\frac{R_{2}}{\sqrt{z^{2}+R_{2}^{2}}}+\frac{R_{1}}{\sqrt{z^{2}+R_{1}^{2}}}\right], \\
& B_{z}^{\mathrm{PC}}(r, z)=\frac{\mu_{0} I}{2\left(R_{2}-R_{1}\right)} \int_{0}^{2 \pi} \frac{d \theta}{2 \pi}\left\{\log \left[\frac{\mathrm{R}_{2}-r \cos (\theta)+\sqrt{z^{2}+R_{2}^{2}+r^{2}-2 R_{2} r \cos (\theta)}}{\mathrm{R}_{1}-r \cos (\theta)+\sqrt{z^{2}+R_{1}^{2}+r^{2}-2 R_{1} r \cos (\theta)}}\right]\right. \\
& \left.-\frac{\mathrm{R}_{2}}{\sqrt{z^{2}+R_{2}^{2}+r^{2}-2 R_{2} r \cos (\theta)}}+\frac{\mathrm{R}_{1}}{\sqrt{z^{2}+R_{1}^{2}+r^{2}-2 R_{1} r \cos (\theta)}}\right\}, \\
& B_{r}^{\mathrm{PC}}(r, z)=\frac{\mu_{0} I z}{2\left(R_{2}-R_{1}\right)} \int_{0}^{2 \pi} \frac{d \theta}{2 \pi} \cos (\theta)\left[\frac{1}{\sqrt{z^{2}+R_{1}^{2}+r^{2}-2 R_{1} r \cos (\theta)}}\right. \\
& \frac{\mathrm{R}_{1}+\sqrt{z^{2}+R_{1}^{2}+r^{2}-2 R_{1} r \cos (\theta)}}{\mathrm{R}_{1}-r \cos (\theta)+\sqrt{z^{2}+R_{1}^{2}+r^{2}-2 R_{1} r \cos (\theta)}}-\frac{1}{\sqrt{z^{2}+R_{2}^{2}+r^{2}-2 R_{2} r \cos (\theta)}} \cdot \\
& \text { - } \left.\frac{\mathrm{R}_{2}+\sqrt{z^{2}+R_{2}^{2}+r^{2}-2 R_{2} r \cos (\theta)}}{\mathrm{R}_{2}-r \cos (\theta)+\sqrt{z^{2}+R_{2}^{2}+r^{2}-R_{2} r \cos (\theta)}}\right] \text {, } \\
& A_{\theta}^{\mathrm{PC}}(r, z)=\frac{\mu_{0} I}{2\left(R_{2}-R_{1}\right)} \int_{0}^{2 \pi} \frac{d \theta}{2 \pi} \cos (\theta)\left\{\sqrt{z^{2}+R_{2}^{2}+r^{2}-2 R_{2} r \cos (\theta)}-\right.\text { (cont.) }
\end{aligned}
$$


$\left.-\sqrt{z^{2}+R_{1}^{2}+r^{2}-2 R_{1} r \cos (\theta)}+r \cos (\theta) \log \left[\frac{\mathrm{R}_{2}-r \cos (\theta)+\sqrt{z^{2}+R_{2}^{2}+r^{2}-2 R_{2} r \cos (\theta)}}{\mathrm{R}_{1}-r \cos (\theta)+\sqrt{z^{2}+R_{1}^{2}+r^{2}-2 R_{1} r \cos (\theta)}}\right]\right\}$.

\section{Periodic Thin Current Layer}

The field of an infinite, periodic current layer is of interest for beam transport. The external field is very different from that of an isolated magnet, particularly at large $r$, where it falls off exponentially rather than as $r^{-3}$. The return flux is diluted to zero intensity in this case.

Let $\ell$ denote an individual solenoid's effective layer length and $g$ denote gap lengths between adjacent wire layers. Assuming the solenoids all have the same polarity, the lattice period length is $P=\ell+g$. For one magnet of the lattice we set

$$
J_{\theta}=S \delta(r-R) \quad-\frac{\ell}{2}<z<\frac{\ell}{2},
$$

and extend this layer periodically. Then the Fourier expansion of $S(z)$ with period $P$ is

$$
S(z)=\frac{S \ell}{P}+\sum_{n=1}^{\infty} S_{n} \cos \left(\frac{2 \pi n z}{P}\right)
$$

with

$$
S_{n}=\frac{2 S}{\pi n} \sin \left(\frac{\pi n \ell}{P}\right)
$$

The longitudinal field can be similarly expanded:

$$
B_{z}=\mu_{0} S \frac{\ell}{P} \Theta(r-R)+\sum_{n=1}^{\infty} B_{n}(r) \cos \left(\frac{2 \pi n z}{P}\right),
$$

with

$$
\begin{array}{ll}
B_{n}(r)=\left(\mu_{0} S\right) \sin \left(\frac{\pi n \ell}{P}\right)\left(\frac{4 R}{P}\right) K_{1}\left(\frac{2 \pi n R}{P}\right) I_{0}\left(\frac{2 \pi n r}{P}\right) & 0<r<R, \\
B_{n}(r)=-\left(\mu_{0} S\right) \sin \left(\frac{\pi n \ell}{P}\right)\left(\frac{4 R}{P}\right) I_{1}\left(\frac{2 \pi n R}{P}\right) K_{0}\left(\frac{2 \pi n r}{P}\right) & R<r<\infty,
\end{array}
$$


where $I_{n}$ and $K_{n}$ are modified Bessel functions of the first and second kind. The functions $B_{n}(r)$ satisfy

$$
\frac{1}{r} \frac{\partial}{\partial r} r \frac{\partial B_{n}}{\partial r}-\left(\frac{2 \pi n}{P}\right)^{2} B_{n}=-\mu_{0} S_{n} \frac{1}{r} \frac{\partial}{\partial r} r \delta(r-R) .
$$

We have used the jump conditions for $B_{n}(r)$ at $R$, which may be derived from eqn. (10.6):

$$
\begin{aligned}
& B_{n}(R+)-B_{n}(R-)=-\mu_{0} S_{n}, \\
& \frac{\partial B_{n}}{\partial r}(R+)-\frac{\partial B_{n}}{\partial r}(R-)=0,
\end{aligned}
$$

and the Bessel function identity,

$$
I_{0}(x) K_{1}(x)+I_{1}(x) K_{0}(x)=\frac{1}{x} .
$$

In similar fashion we find

$$
\begin{array}{ll}
B_{r}=\sum_{n=1}^{\infty} C_{n}(r) \sin \left(\frac{2 \pi n z}{P}\right) \\
C_{n}(r)=\left(\mu_{0} S\right) \sin \left(\frac{\pi n \ell}{P}\right)\left(\frac{4 R}{P}\right) K_{1}\left(\frac{2 \pi n R}{P}\right) I_{1}\left(\frac{2 \pi n r}{P}\right) & 0<r<R \\
C_{n}(r)=\left(\mu_{0} S\right) \sin \left(\frac{\pi n \ell}{P}\right)\left(\frac{4 R}{P}\right) I_{1}\left(\frac{2 \pi n R}{P}\right) K_{1}\left(\frac{2 \pi n r}{P}\right) & R<r<\infty
\end{array}
$$

This solves $\vec{\nabla} \cdot \vec{B}=0$ with $B_{z}$ given above. The components $C_{n}(r)$ are continuous at $r=R$.

At large $r$ the asymptotic expansion of $K_{0}(2 \pi n r / P)$ gives

$$
B_{n} \sim \frac{\exp (-2 \pi \mathrm{n} \mathrm{r} / \mathrm{P})}{\sqrt{r}}
$$

showing the asserted exponential decrease of field strength outside the current layer.

However, the calculated net flux in this example is $\mu_{0} S \pi R^{2} \ell / P$, so the return flux must 
be regarded as being diluted to zero magnitude. Plots of $B_{z}$ and $B_{r}$ at various $r$ are presented below for the parameters:

$$
\mu_{0} S \frac{\ell}{P}=10.0 \mathrm{~T}, R=.2 \mathrm{~m}, \quad \ell=.8 \mathrm{~m}, \quad g=.2 \mathrm{~m}, P=\ell+g=1.0 \mathrm{~m} .
$$

A total of 200 terms are used in the Fourier expansion. Note that while $B_{z}$ reaches $\sim 7.0 \mathrm{~T}$ in the gaps, it drops to less than $.5 \mathrm{~T}$ in the volume behind the wire where an induction core could be located. This calculation is modified in section 18 with the addition of a permeable yoke.
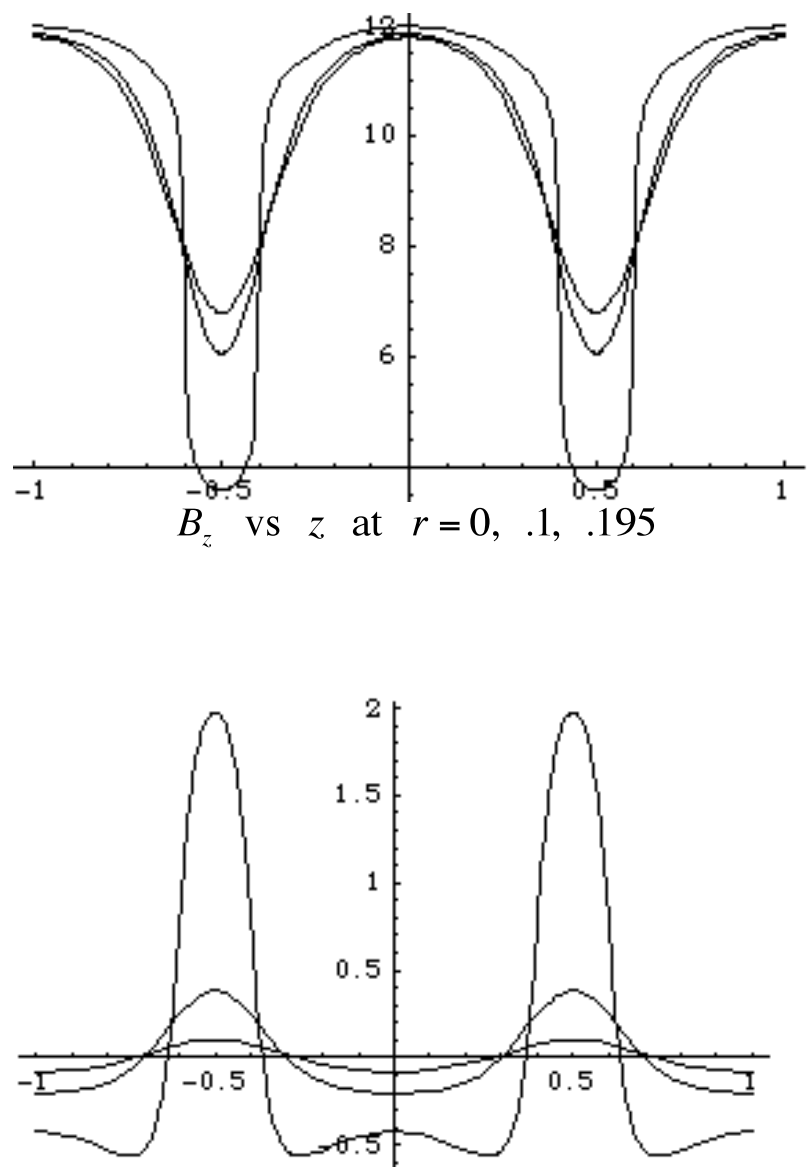

$$
B_{z} \text { vs } z \text { at } r=.25, .4, .55
$$




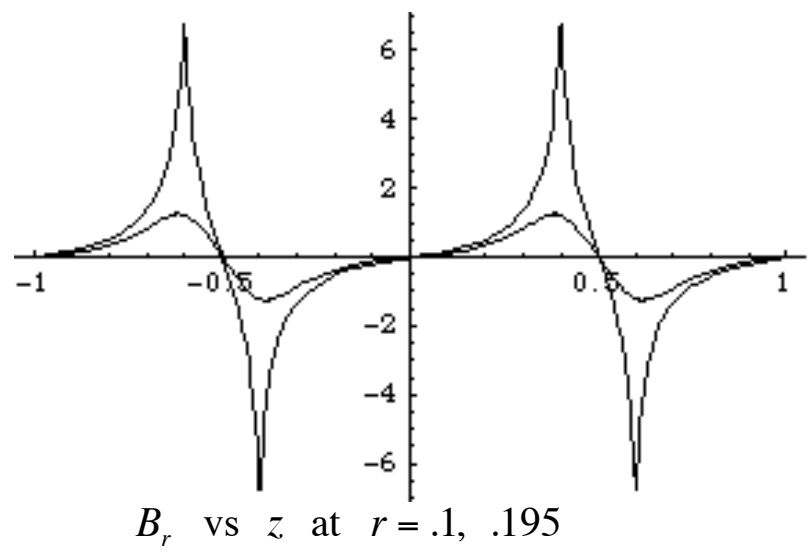

\section{Far Field and External Field}

Because a solenoid system may have a large longitudinal dipole moment, its far field can interfere with nearby experiments and the operation of accelerator components. A short magnetic bend resembles a solenoid turned on its side, but a permeable yoke is usually used to confine its return flux and eliminate the far field. This technique is not as effective for a transport solenoid because the yoke must not block the vacuum bore. Also, if annular yokes are used in an induction linac they increase the diameter of adjacent induction cores and thereby increase the overall cost. It is possible to greatly reduce the far field by alternating the polarity of solenoids, but this may reduce their effectiveness for beam transport and actually increase the external field (just outside the wire). We are therefore motivated to evaluate the far and external fields without return flux confinement.

The far field and external field can be approximated using two different series expansions of the global potential $\varphi$. Both expansions may have contributions from several or many individual magnets, so it may be misleading to consider only one magnet at a time. Recall from section 5 ,

$$
\vec{B}=\mu_{0} S(r, z) \hat{e}_{z}+\vec{\nabla} \varphi,
$$

where $\mathrm{S}(\mathrm{r}, \mathrm{z})$ is the cumulative current distribution:

$$
S(r, z)=\int_{r}^{\infty} d r^{\prime} J_{\theta}\left(r^{\prime}, z\right)
$$

The Green function solution for $\varphi$ is, from section 6 :

$$
\varphi=\frac{\mu_{0}}{4 \pi} \int_{-\infty}^{+\infty} d z^{\prime} \int_{0}^{\infty} d r^{\prime} r^{\prime} \int_{0}^{2 \pi} d \theta^{\prime} \frac{\partial S^{\prime}}{\partial z^{\prime}} \frac{1}{\sqrt{\left(z^{\prime}-z\right)^{2}+r^{\prime 2}+r^{2}-2 r^{\prime} r \cos \left(\theta^{\prime}-\theta\right)}} .
$$




\subsection{Far field expansion}

We first consider the far field expansion, which is in inverse powers of $|\vec{r}|=\left(r^{2}+z^{2}\right)^{1 / 2}$, with $r$ and $z$ both treated as large on the order $|\vec{r}|$, which is larger than $\left|\overrightarrow{r^{\prime}}\right|$. We could laboriously expand $\left|\overrightarrow{r^{\prime}}-\vec{r}\right|^{-1}$ in inverse powers of $|\vec{r}|$, work the elementary integrals over $\theta^{\prime}$, and gather terms of the same order. Fortunately this task has already been done for us in texts that treat electrostatics using spherical harmonics. For $|\vec{r}|>\left|\overrightarrow{r^{\prime}}\right|$,

$$
\int_{0}^{2 \pi} \frac{d \theta}{2 \pi} \frac{1}{\left|\overrightarrow{r^{\prime}}-\vec{r}\right|}=\sum_{n=0}^{\infty} \frac{\left|\overrightarrow{r^{\prime}}\right|^{n}}{|\vec{r}|^{n+1}} P_{n}(\cos \psi) P_{n}\left(\cos \psi^{\prime}\right)
$$

where

$$
\cos (\psi) \equiv \frac{z}{|\vec{r}|}, \quad \cos \left(\psi^{\prime}\right) \equiv \frac{z^{\prime}}{\left|\overrightarrow{r^{\prime}}\right|},
$$

and the $P_{n}$ are the Legendre polynomials:

$$
\begin{aligned}
& P_{0}(x)=1, \\
& P_{1}(x)=x, \\
& P_{2}(x)=\left(3 x^{2}-1\right) / 2, \\
& P_{3}(x)=\left(5 x^{3}-3 x\right) / 2, \\
& P_{4}(x)=\left(35 x^{4}-30 x^{2}+3\right) / 8, \\
& P_{5}(x)=\left(63 x^{5}-70 x^{3}+15 x\right) / 8, \text { etc. }
\end{aligned}
$$

Inserting expression (11.4) into eqn. (11.3) gives

$$
\varphi=\frac{\mu_{0}}{2} \int_{-\infty}^{+\infty} d z^{\prime} \int_{0}^{\infty} d r^{\prime} r^{\prime} \frac{\partial S^{\prime}}{\partial z^{\prime}}\left\{\frac{1}{|\vec{r}|}+\frac{z^{\prime} z}{|\vec{r}|^{3}}+\frac{\left(2 z^{2}-r^{2}\right)\left(2 z^{\prime 2}-r^{\prime 2}\right)}{4|\vec{r}|^{5}}+\frac{\left(2 z^{3}-3 z r^{2}\right)\left(2 z^{\prime 3}-3 z^{\prime} r^{\prime 2}\right)}{4|\vec{r}|^{7}}+\ldots\right\} .
$$

The leading (monopole) term vanishes unless $S^{\prime}$ does not vanish as $\left|z^{\prime}\right| \rightarrow \infty$. After integration by parts in $z^{\prime}$ eqn. (11.7) becomes 


$$
\begin{aligned}
\varphi=-\frac{\mu_{0}}{2} \int_{-\infty}^{+\infty} d z^{\prime} \int_{0}^{\infty} d r^{\prime} r^{\prime} S^{\prime}\left\{\frac{z}{|\vec{r}|^{3}}+\frac{\left(2 z^{2}-r^{2}\right) z^{\prime}}{|\vec{r}|^{5}}+\frac{\left(2 z^{3}-3 z r^{2}\right)\left(6 z^{\prime 2}-3 r^{\prime 2}\right)}{4|\vec{r}|^{7}}+\ldots\right\} \\
=\varphi_{1}+\varphi_{2}+\varphi_{3}+\ldots
\end{aligned}
$$

Equation (11-8) may be written in the compact form

$$
\varphi=-\frac{\mu_{0}}{4 \pi}\left[\frac{m_{1} P_{1}(\cos \psi)}{|\vec{r}|^{2}}+\frac{m_{2} P_{2}(\cos \psi)}{|\vec{r}|^{3}}+\frac{m_{3} P_{3}(\cos \psi)}{|\vec{r}|^{4}}+\ldots\right],
$$

where the $m_{i}$ are the multipole moments:

$$
\begin{aligned}
& m_{1}=2 \pi \int_{-\infty}^{+\infty} d z \int_{0}^{\infty} d r r S(r, z), \\
& m_{2}=2 \pi \int_{-\infty}^{+\infty} d z \int_{0}^{\infty} d r r S(r, z)(2 z), \\
& m_{3}=2 \pi \int_{-\infty}^{+\infty} d z \int_{0}^{\infty} d r r S(r, z) \frac{3\left(2 z^{2}-r^{2}\right)}{2},
\end{aligned}
$$

etc. Here $m_{1}$ is the total dipole moment with the conventional definition given in section 2. The second $\left(m_{2}\right)$ and third $\left(m_{3}\right)$ moments have been defined in a way that makes eqn. (11-10) simple in appearance.

For the far field expansion to be useful we must specify a coordinate origin, which should be at the magnet system center in some weighted sense. For a single lens the origin would usually be at the lens center. More generally the quadrupole moment can usually be zeroed by the right choice of origin. However, if $m_{1}$ vanishes, then $m_{2}$ is independent of the coordinate origin. In this case $m_{3}$ can be made to vanish.

Using $\vec{B}^{\text {residual }}=\vec{\nabla} \varphi$, the dipole components of the far field are

$$
\begin{aligned}
& B_{r}^{f a r}=-\frac{\partial}{\partial r} \frac{\mu_{0}}{4 \pi} \frac{m_{1} z}{{\sqrt{r^{2}+z^{2}}}^{3}}=\frac{\mu_{0}}{4 \pi} \frac{3 m_{1} z r}{{\sqrt{r^{2}+z^{2}}}^{5}}, \\
& B_{z}^{f a r}=-\frac{\partial}{\partial z} \frac{\mu_{0}}{4 \pi} \frac{m_{1} z}{{\sqrt{r^{2}+z^{2}}}^{3}}=+\frac{\mu_{0}}{4 \pi} \frac{m_{1}\left(2 z^{2}-r^{2}\right)}{{\sqrt{r^{2}+z^{2}}}^{5}} .
\end{aligned}
$$


A solenoid with current density $J_{\theta}(r)$ between ends at $z= \pm \ell / 2$ has only odd order magnetic moments. If this current density is also constant between radii $\mathrm{R}_{1}$ and $\mathrm{R}_{2}$, i.e. $J_{\theta}=S /\left(R_{2}-R_{1}\right)$, then the two lowest magnetic moments are

$$
\begin{aligned}
& m_{1}=\frac{\pi \ell S}{3} \frac{R_{2}^{3}-R_{1}^{3}}{R_{2}-R_{1}} \\
& m_{3}=\pi \ell S\left(\frac{\ell^{2}}{12} \frac{R_{2}^{3}-R_{1}^{3}}{R_{2}-R_{1}}-\frac{3}{20} \frac{R_{2}^{5}-R_{1}^{5}}{R_{2}-R_{1}}\right) .
\end{aligned}
$$

These expressions can still be used if the solenoid is centered at $z=z_{0}$ by substituting $z \rightarrow z-z_{0}$ in field expressions such as eqn. $(11.12 \mathrm{a}, \mathrm{b})$. Note that $m_{3}=0$ if $\left(R_{2}^{5}-R_{1}^{5}\right) /\left(R_{2}^{3}-R_{1}^{3}\right)=5 \ell^{2} / 9$. For a thin layer this is $R=\ell / \sqrt{3}$.

\subsection{External field expansion}

To obtain an expansion for the external field we take $r^{\prime}<r$, but make no assumption about $z$ and $z^{\prime}$. Then we write equation (11.3) as

$$
\begin{gathered}
\varphi=\frac{\mu_{0}}{2} \int_{-\infty}^{+\infty} d z^{\prime} \int_{0}^{\infty} d r^{\prime} r^{\prime} \frac{\partial S^{\prime}}{\partial z^{\prime}} \frac{1}{\sqrt{\left(z^{\prime}-z\right)^{2}+r^{2}}} \int_{0}^{2 \pi} \frac{d \theta^{\prime}}{2 \pi} \frac{1}{\sqrt{1-\left[\frac{2 r^{\prime} r \cos \left(\theta^{\prime}\right)-r^{\prime 2}}{\left(z^{\prime}-z\right)^{2}+r^{2}}\right]}} \\
=\frac{\mu_{0}}{2} \int_{-\infty}^{+\infty} d z^{\prime} \int_{0}^{\infty} d r^{\prime} r^{\prime} \frac{\partial S^{\prime}}{\partial z^{\prime}} \frac{1}{\sqrt{\left(z^{\prime}-z\right)^{2}+r^{2}}} \int_{0}^{2 \pi} \frac{d \theta^{\prime}}{2 \pi} \cdot \\
\bullet\left\{1+\frac{1}{2}[\ldots]+\frac{3}{8}[\ldots]^{2}+\frac{5}{16}[\ldots]^{3}+\frac{35}{128}[\ldots]^{4}+\ldots\right\},
\end{gathered}
$$

where $[\ldots]$ denotes the bracketed expression in eqn. (11.14). After the integrals over $\theta^{\prime}$ are worked and terms of like order in $r^{\prime}$ are grouped we have

$$
\begin{aligned}
& \varphi=\frac{\mu_{0}}{2} \int_{-\infty}^{+\infty} d z^{\prime} \int_{0}^{\infty} d r^{\prime} r^{\prime} \frac{\partial S^{\prime}}{\partial z^{\prime}} \bullet \\
& \bullet\left\{\frac{1}{\sqrt{\left(z^{\prime}-z\right)^{2}+r^{2}}}+r^{\prime 2}\left[-\frac{1}{2} \frac{1}{\sqrt{\ldots}^{3}}+\frac{3}{4} \frac{r^{2}}{\sqrt{\ldots}^{5}}\right]+r^{\prime 4}\left[\frac{3}{8} \frac{1}{\sqrt{\ldots}^{5}}-\frac{15}{8} \frac{r^{2}}{\sqrt{\ldots}^{7}}+\frac{105}{64} \frac{r^{4}}{\sqrt{\ldots}^{9}}\right]+\ldots\right\} .
\end{aligned}
$$

The radial moments defined in section 2 may now be used: 


$$
\begin{aligned}
& S(z) \overline{r^{2}}(z)=2 \int d r^{\prime} r^{\prime} S\left(r^{\prime}, z\right)=\int d r^{\prime} r^{\prime 2} J_{\theta}\left(r^{\prime}, z\right) \\
& S(z) \overline{r^{4}}(z)=4 \int d r^{\prime} r^{\prime 3} S\left(r^{\prime}, z\right)=\int d r^{\prime} r^{4} J_{\theta}\left(r^{\prime}, z\right) \\
& S(z) \overline{r^{6}}(z)=6 \int d r^{\prime} r^{5} S\left(r^{\prime}, z\right)=\int d r^{\prime} r^{\prime 6} J_{\theta}\left(r^{\prime}, z\right) \\
& \varphi=\frac{\mu_{0}}{2} \int_{-\infty}^{+\infty} d z^{\prime}\left\{\left[\frac{\partial}{\partial z^{\prime}} \frac{S\left(z^{\prime}\right) \overline{r^{2}}\left(z^{\prime}\right)}{2}\right] \cdot \frac{1}{\sqrt{\left(z^{\prime}-z\right)^{2}+r^{2}}}+\left[\frac{\partial}{\partial z^{\prime}} \frac{S\left(z^{\prime}\right) \overline{r^{4}}\left(z^{\prime}\right)}{4}\right] \bullet\right. \\
& \left.\left[-\frac{1}{2} \frac{1}{\sqrt{\ldots}^{3}}+\frac{3}{4} \frac{r^{2}}{\sqrt{\ldots}^{5}}\right]+\left[\frac{\partial}{\partial z^{\prime}} \frac{S\left(z^{\prime}\right) \overline{r^{6}}\left(z^{\prime}\right)}{6}\right] \bullet\left[\frac{3}{8} \frac{1}{\sqrt{\sqrt{n}^{5}}}-\frac{15}{8} \frac{r^{2}}{\sqrt{\sqrt{n}^{7}}}+\frac{105}{64} \frac{r^{4}}{\sqrt{\sqrt{n}^{9}}}\right]+\ldots\right\} \text {. }
\end{aligned}
$$

This series appears to be complicated, but in fact we have again generated Legendre polynomials. Let

$$
\cos (\gamma) \equiv \frac{\left(z^{\prime}-z\right)}{\sqrt{\left(z^{\prime}-z\right)^{2}+r^{2}}}
$$

Then eqn. (11.18) can be written

$$
\begin{aligned}
\varphi=\frac{\mu_{0}}{2} \int_{-\infty}^{+\infty} d z^{\prime} & \left\{\frac{P_{0}(\cos \gamma)}{\sqrt{\left(z^{\prime}-z\right)^{2}+r^{2}}}\left[\frac{\partial}{\partial z^{\prime}} \frac{S\left(z^{\prime}\right) \overline{r^{2}\left(z^{\prime}\right)}}{2}\right]\right. \\
& \left.-\frac{1}{2} \frac{P_{2}(\cos \gamma)}{\sqrt{\ldots}^{3}}\left[\frac{\partial}{\partial z^{\prime}} \frac{S\left(z^{\prime}\right) \overline{r^{4}\left(z^{\prime}\right)}}{4}\right]+\frac{3}{8} \frac{P_{4}(\cos \gamma)}{\sqrt{\ldots}^{5}}\left[\frac{\partial}{\partial z^{\prime}} \frac{S\left(z^{\prime}\right) \overline{r^{6}\left(z^{\prime}\right)}}{6}\right]+\ldots\right\} .
\end{aligned}
$$

We treat the semi-infinite distribution

$$
S(r, z)=S(r) \Theta(z)
$$

with $S(r=0) \equiv S$. From eqn. (11.18),

$$
\varphi=\frac{-\mu_{0} S}{2}\left\{\frac{\overline{r^{2}}}{2} \frac{1}{|\vec{r}|}+\frac{\overline{r^{4}}}{4}\left[\frac{-1}{2|\vec{r}|^{3}}+\frac{3 r^{2}}{4|\vec{r}|^{5}}\right]+\frac{\overline{r^{6}}}{6}\left[\frac{3}{8|\vec{r}|^{5}}-\frac{15 r^{2}}{8|\vec{r}|^{7}}+\frac{105 r^{4}}{64|\vec{r}|^{9}}\right]+\ldots\right\}
$$

These terms are proportional to the radial moments at the current layer end and include a monopole term. For a thin annular layer at radius R,

$$
\overline{r^{2}}=R^{2}, \overline{r^{4}}=R^{4}, \overline{r^{6}}=R^{6}, \ldots
$$


For a uniform thick layer $\left(R_{1} \leq r \leq R_{2}\right)$ :

$$
\overline{r^{2}}=\frac{R_{2}^{3}-R_{1}^{3}}{3\left(R_{2}-R_{1}\right)}, \overline{r^{4}}=\frac{R_{2}^{5}-R_{1}^{5}}{5\left(R_{2}-R_{1}\right)}, \overline{r^{6}}=\frac{R_{2}^{7}-R_{1}^{7}}{7\left(R_{2}-R_{1}\right)}, \ldots
$$

The leading (monopole) term of eqn. (11.22) gives

$$
\vec{B}^{e x}=\vec{\nabla} \varphi \approx \frac{\mu_{0} S \overline{r^{2}}}{4} \frac{\vec{r}}{|\vec{r}|^{3}}=(\operatorname{direct} \text { flux }) \cdot \frac{\vec{r}}{4 \pi|\vec{r}|^{3}},
$$

as expected. In general, far away from $z=0$, the first two terms from eqn. (11.22) plus the direct field give

$$
\vec{B} \approx \mu_{0} S(r) \Theta(z) \hat{e}_{z}+\frac{\mu_{0} S}{2}\left\{\frac{\overline{r^{2}}}{2} \frac{\vec{r}}{|\vec{r}|^{3}}-\frac{\overline{r^{4}}}{4}\left[\frac{3 \vec{r}}{2|\vec{r}|^{5}}-\frac{15 r^{2} \vec{r}}{4|\vec{r}|^{7}}+\frac{3 r \hat{e}_{r}}{2|\vec{r}|^{5}}\right]\right\} .
$$

\section{Field in the Wire Layer}

We now have several ways to calculate the field within the wire layer. Near the layer ends an accurate calculation requires the general formalism of section 6 or the thick layer solution (equation (9.3)), plus contributions from nearby magnets. Away from a layer end the external field expansion of section 11 plus the direct field, $\mu_{0} S(r, z) \hat{e}_{z}$, is applicable, e.g. eqn (11.26).

The peak field in the wire layer is of great interest since it limits the engineering averaged critical current density - see sections 2 and 20. Usually this peak field occurs at the inner edge of the wire layer at the middle of a magnet. The logarithmic singularity of $B_{r}$ at the ends of a thin layer is usually not representative of realistic cases. The peak field can be accurately estimated using the first two terms of the near-axis expansion if the magnet is not too short $(R / \ell \leq .5)$ :

$$
\begin{aligned}
& B_{z} \approx B_{0}(z)-B_{0}^{\prime \prime}(z) \frac{r^{2}}{4}, \\
& B_{r} \approx-B_{0}^{\prime}(z) \frac{r}{2}+B_{0}^{\prime \prime \prime}(z) \frac{r^{3}}{16} .
\end{aligned}
$$


We use the thick layer formula for a single magnet, eqn. (9.5), to obtain $B_{0}$ and $B_{0}{ }^{\prime \prime}$ at the magnet center $(\mathrm{z}=0)$, and by symmetry $B_{r}$ vanishes there. At the inner wire radius $R_{1}$ this gives for a magnet of length $\ell$ :

$$
\begin{gathered}
B_{\max } \cong B_{0}(0)-\frac{B_{0}^{\prime \prime}(0) R_{1}^{2}}{4}=\frac{\mu_{0} S}{2} \frac{\ell}{R_{2}-R_{1}} \bullet \\
\text { - }\left\{\log \left[\frac{2 R_{2} / \ell+\sqrt{1+\left(2 R_{2} / \ell\right)^{2}}}{2 R_{1} / \ell+\sqrt{1+\left(2 R_{1} / \ell\right)^{2}}}\right]+\frac{1}{4}\left(\frac{2 R_{1}}{\ell}\right)^{2}\left[\frac{\left(2 R_{2} / \ell\right)^{3}}{{\sqrt{1+\left(2 R_{2} / \ell\right)^{2}}}^{3}}-\frac{\left(2 R_{1} / \ell\right)^{3}}{{\sqrt{1+\left(2 R_{1} / \ell\right)^{2}}}^{3}}\right]\right\} .
\end{gathered}
$$

This formula may be written in dimensionless form using the definitions

$$
\begin{aligned}
& \bar{R}=\frac{R_{1}+R_{2}}{2}, \Delta=\frac{R_{2}-R_{1}}{\bar{R}}, \alpha=2 \bar{R} / \ell \\
& B_{\max }^{\mathrm{approx}} \cong\left(\mu_{0} S\right) \frac{1}{\alpha \Delta}\left\{\log \left[\frac{\alpha\left(1+\frac{\Delta}{2}\right)+\sqrt{1+\alpha^{2}\left(1+\frac{\Delta}{2}\right)^{2}}}{\alpha\left(1-\frac{\Delta}{2}\right)+\sqrt{1+\alpha^{2}\left(1-\frac{\Delta}{2}\right)^{2}}}\right]\right. \\
& \left.+\frac{1}{4} \alpha^{5}\left(1-\frac{\Delta}{2}\right)^{2}\left[\frac{\left(1+\frac{\Delta}{2}\right)^{3}}{\sqrt{1+\alpha^{2}\left(1+\frac{\Delta}{2}\right)^{2}}}-\frac{\left(1-\frac{\Delta}{2}\right)^{3}}{\sqrt{1+\alpha^{2}\left(1-\frac{\Delta}{2}\right)^{2}}}\right]\right\}
\end{aligned}
$$

The accuracy of eqn. (12.4) is indicated in the following table, where the ratio $B_{\max } / B_{\max }^{\text {approx }}$ is given for several values of $\Delta$ and $\alpha$.

\begin{tabular}{|l|l|l|l|}
\hline$\Delta \backslash \alpha$ & 1.0 & .5 & .25 \\
\hline 1 & .998320 & .999746 & .999990 \\
\hline .5 & .992932 & .998618 & .999948 \\
\hline .25 & .987348 & .997435 & .999904 \\
\hline .10 & .982344 & .996471 & .999868 \\
\hline .04 & .979903 & .996029 & .999851 \\
\hline
\end{tabular}

The exact values of $B_{\max } / \mu_{0} S$ for several values of $\alpha$ and $\Delta$ are computed and tabulated below using 


$$
\begin{gathered}
B_{\max }=\frac{\mu_{0} S}{R_{2}-R_{1}} \int_{R_{1}}^{R_{2}} d R \int \frac{d \theta}{2 \pi}\left[\frac{R^{2}-R R_{1} \cos (\theta)}{R^{2}+R_{1}^{2}-2 R R_{1} \cos (\theta)}\right] \bullet \\
\bullet \frac{\ell / 2}{\sqrt{\ell^{2} / 4+R^{2}+R_{1}^{2}-2 R R_{1} \cos (\theta)}} .
\end{gathered}
$$

Values of $B_{\max } / \mu_{0} S$ for given $\Delta$ and $\alpha$

\begin{tabular}{|l|l|l|l|}
\hline$\Delta \backslash \alpha$ & 1.0 & .5 & .25 \\
\hline 1.0 & .742700 & .897437 & .968828 \\
\hline .5 & .776529 & .907269 & .971029 \\
\hline .25 & .798374 & .912353 & .971858 \\
\hline .10 & .812152 & .915356 & .972267 \\
\hline .04 & .817666 & .916536 & .972412 \\
\hline
\end{tabular}

\section{Current Distribution to Produce a Given Field}

When designing a magnet system one usually asks for a current distribution to produce a desired field. For solenoids it is clear that any on-axis field profile with $B \leq 10 T$ can be made with scale length variations down to a few centimeters. But for beam transport and focusing this may also require an unacceptably small radius for the current layer. Increasing the current layer radius increases the scale length of field variations. In the present calculation we assume the current is concentrated in a thin layer of fixed radius $R ; J_{\theta}=S(z) \delta(r-R)$. For given $B_{0}(z)$ it is always possible to solve for $S(z)$, which provides guidance for a configuration of actual lenses. The field at the wire layer may be unacceptably large if the variations of $B_{0}(z)$ have scale length smaller than $R$, however this problem may be evaded in some applications by using small radius solenoids where the beam radius is small.

Recall the thin layer formula for the on-axis field - see section 3,

$$
B_{0}(z)=\frac{\mu_{0} R^{2}}{2} \int_{-\infty}^{+\infty} d z^{\prime} \frac{S\left(z^{\prime}\right)}{{\sqrt{\left(z^{\prime}-z\right)^{2}+R^{2}}}^{3}}
$$

Defining the Fourier integral transformations

$$
\left[\tilde{B}_{0}(k), \tilde{S}(k)\right]=\int_{-\infty}^{+\infty} d z e^{-i k z}\left[B_{0}(z), S(z)\right],
$$

we get from eqn. (13.1) 


$$
\begin{aligned}
& \tilde{B}_{0}(k)=\frac{\mu_{0} R^{2}}{2} \int_{-\infty}^{+\infty} d z^{\prime} S\left(z^{\prime}\right) \int_{-\infty}^{+\infty} d z \frac{e^{-i k z}}{{\sqrt{\left(z^{\prime}-z\right)^{2}+R^{2}}}^{3}} \\
& =\frac{\mu_{0}}{2} \int_{-\infty}^{+\infty} d z^{\prime} e^{-i k z^{\prime}} S\left(z^{\prime}\right) \int_{-\infty}^{+\infty} d x \frac{e^{-i k R x}}{{\sqrt{x^{2}+1}}^{3}},
\end{aligned}
$$

with $x=\left(z-z^{\prime}\right) / R$. Inserting the identity

$$
\int_{0}^{\infty} d x \frac{\cos (k R x)}{{\sqrt{x^{2}+1}}^{3}}=|k R| K_{1}(k R \mid)
$$

gives the general relation

$$
\tilde{B}_{0}(k)=\mu_{0} \tilde{S}(k)|k R| K_{1}(|k R|),
$$

where $K_{1}$ is the modified Bessel function. Equation (13.5) gives $\tilde{S}$ for a thin layer of radius $R$ if $\tilde{B}_{0}$ is derived from a given on-axis field.

For the specific example,

$$
B_{0}(z)=B_{00} e^{-z^{2} / L^{2}},
$$

the transform of $B_{0}(z)$ and inversion for $S(z)$ are

$$
\begin{aligned}
& \tilde{B}_{0}(k)=B_{00} \sqrt{\pi} L e^{-k^{2} L^{2} / 4}, \\
& \mu_{0} S(z)=\int_{-\infty}^{+\infty} \frac{d k}{2 \pi} e^{i k z} \mu_{0} \tilde{S}(k)=\int_{-\infty}^{+\infty} \frac{d k}{2 \pi} e^{i k z} \frac{B_{00} \sqrt{\pi} L e^{-k^{2} L^{2} / 4}}{(|k| R) K_{1}(|k| R)} .
\end{aligned}
$$

Setting $y=k L$ gives the convenient form for computations:

$$
\frac{\mu_{0} S(z)}{B_{00}}=\frac{1}{\sqrt{\pi}} \frac{L}{R} \int_{0}^{\infty} d y \frac{e^{-y^{2} / 4} \cos (y z / L)}{y K_{1}(y R / L)} .
$$

For $R / L \rightarrow 0$ and $|z| / L$ not too large, this formula goes over to the expected form (using $\left.x K_{1}(x) \rightarrow 1\right)$,

$$
\mu_{0} S(z) \rightarrow B_{00} e^{-z^{2} / L^{2}}=B_{0}(z) .
$$

However for finite $R / L, \mu_{0} S(z)$ exceeds $B_{00}$ at $z=0$, e.g. by a factor of 2.5 when $R / L=1.0$. Also, oscillations of $S(z)$ appear; these provide guidance for the layout of 
solenoids that can produce a desired on-axis field. The following figures plot $\mu_{0} S(z) / B_{00}$ for various values of $R$, with $\mathrm{L}=1.0$.
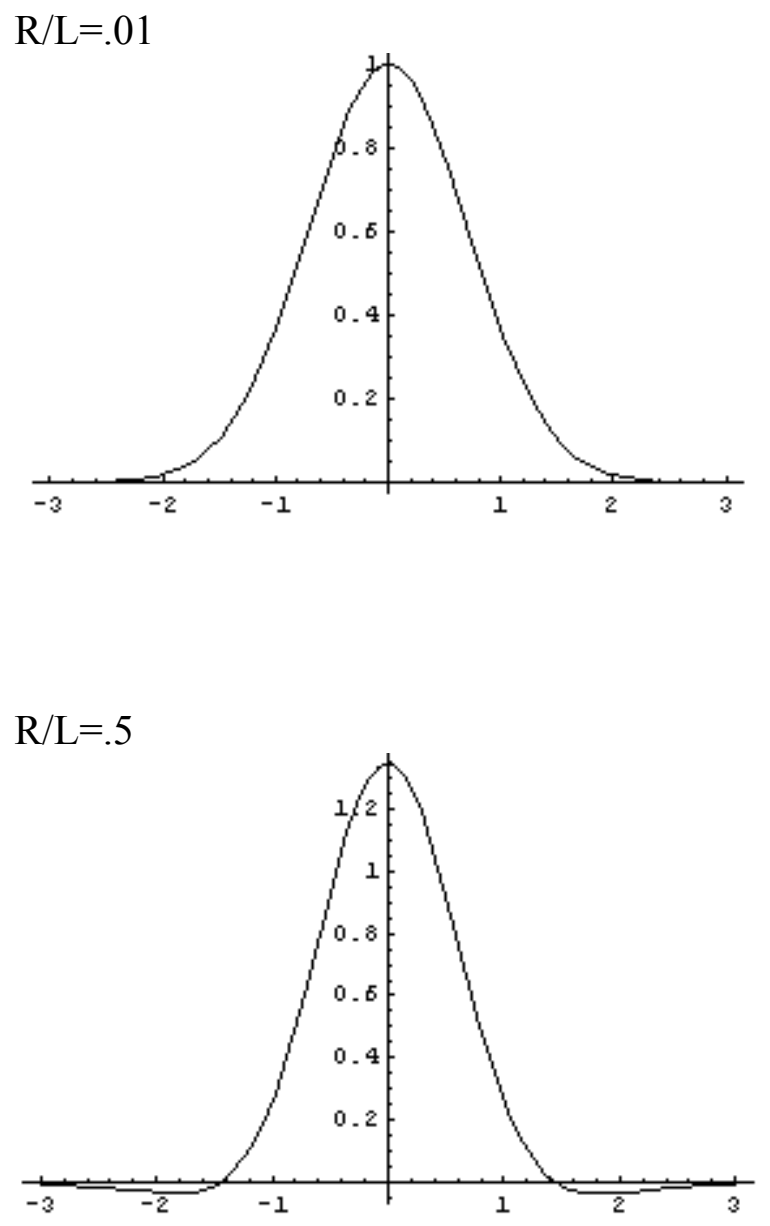

$\mathrm{R} / \mathrm{L}=1$

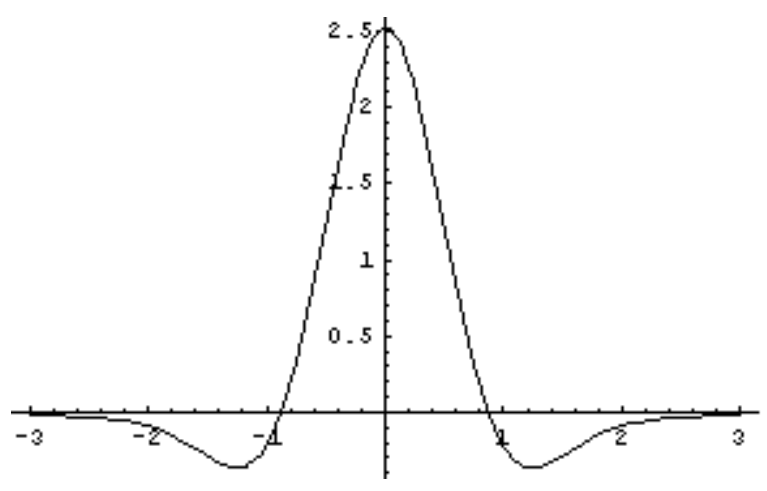


$\mathrm{R} / \mathrm{L}=2$

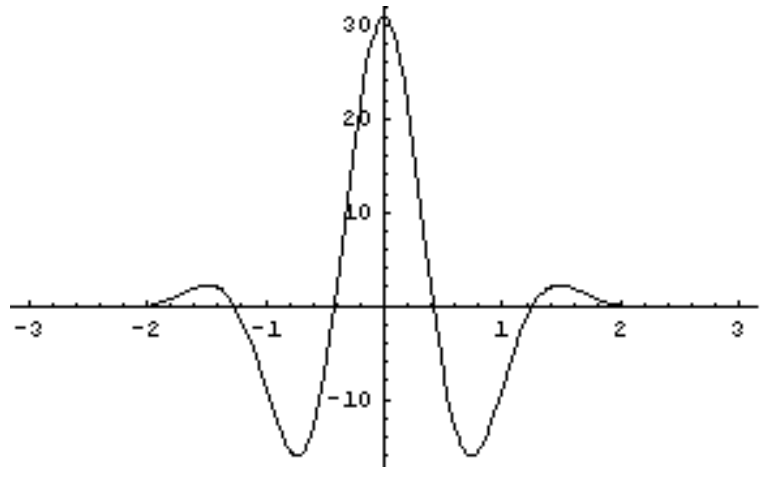

\section{Misaligned and Tilted Solenoid}

So far we have only treated axisymmetric systems, with field components $B_{r}(r, z)$ and $B_{z}(r, z)$. There are always small deviations from this convenient symmetry because of imperfections in design and manufacture, stress, and positioning errors (misalignment and tilt). These can have a significant effect on a transported ion beam, so it is necessary to specify maximum allowed deviations and make precise field measurements. However, the restricted problem of the effect of positioning errors on the field can be treated analytically as presented below.

The field of a single magnet is considered to be ideal and perfectly characterized in its own coordinate frame. It greatly simplifies the formalism to use Cartesian coordinates. For example if $B_{0}(z)$ is the known on-axis field in the absence of a positioning error, then

$$
\begin{aligned}
& B_{z}^{I}(x, y, z)=B_{0}(z)-B_{0}^{\prime \prime}(z) \frac{x^{2}+y^{2}}{4}+\ldots \\
& B_{x}^{I}(x, y, z)=-\frac{B_{0}^{\prime}(z) x}{2}+\frac{B_{0}^{\prime \prime \prime}(z) x\left(x^{2}+y^{2}\right)}{16}-\ldots \\
& B_{y}^{I}(x, y, z)=-\frac{B_{0}^{\prime}(z) y}{2}+\frac{B_{0}^{\prime \prime \prime}(z) y\left(x^{2}+y^{2}\right)}{16}-\ldots
\end{aligned}
$$

is the magnet's ideal near-axis field.

A misalignment is simply a translation of the magnet center by the vector

$$
\vec{T}=\Delta_{x} \hat{e}_{x}+\Delta_{y} \hat{e}_{y}+\Delta_{z} \hat{e}_{z},
$$


with displacements $\Delta_{i}$ typically less than $\pm 10^{-3} \mathrm{~m}$. The misaligned field is obtained from eqn. (14-1) simply by substituting the displacement:

$$
\vec{B}=\vec{B}^{\mathrm{I}}\left(x-\Delta_{x}, y-\Delta_{y}, z-\Delta_{z}\right)
$$

A second type of positioning error is a tilt, which holds a magnet's center fixed and orients its axis at an angle $\alpha$ with respect to the system axis, typically with $\alpha$ less than $10^{-3}$ radians. The tilted magnet axis points in the direction of the unit vector

$$
\hat{e}=\sin (\alpha) \cos (\beta) \hat{e}_{x}+\sin (\alpha) \sin (\beta) \hat{e}_{y}+\cos (\alpha) \hat{e}_{z},
$$

where $\beta$ is the angle of the projection of $\hat{e}$ in the $x-y$ plane. The two angles $(\alpha, \beta)$ are the polar coordinates of $\hat{e}$, the usual symbols $(\theta, \varphi)$ having been used for other quantities. A net azimuthal rotation of a magnet around its own axis does not need to be considered since it does not change the field.

The coordinates of a fixed point in the system frame are denoted by $(x, y, z)$ and in the translated and rotated magnet frame by $(X, Y, Z)$. These coordinate sets are related by the inhomogeneous linear transformation

$$
\left(\begin{array}{l}
X \\
Y \\
Z
\end{array}\right)=\left(\begin{array}{l}
R_{11} R_{12} R_{13} \\
R_{21} R_{22} R_{23} \\
R_{31} R_{32} R_{33}
\end{array}\right)\left(\begin{array}{l}
x-\Delta_{x} \\
y-\Delta_{y} \\
z-\Delta_{z}
\end{array}\right)
$$

where $\left(R_{i j}\right)$ is the orthogonal matrix

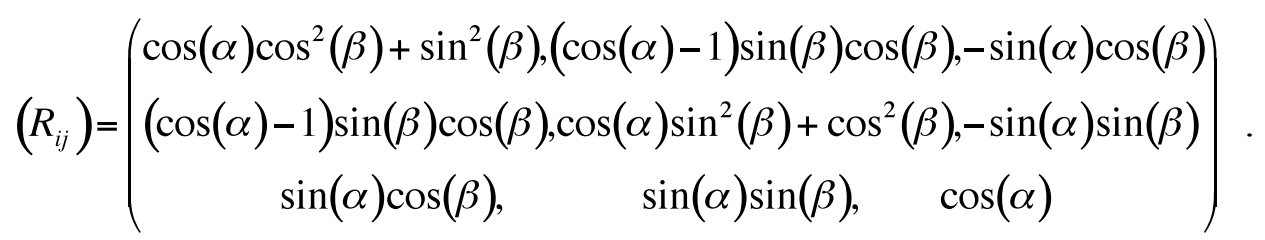

Eqn. (14.6) may be derived by multiplying the matrices for successive rotations of the magnet's coordinate frame through angles $\beta, \alpha$, and $-\beta$.

The field components of the rotated and translated magnet are then found by applying the transpose of $\left(R_{i j}\right)$ :

$$
\begin{aligned}
& B_{x}(x, y, z)=R_{11} B_{x}^{I}(X, Y, Z)+R_{21} B_{y}^{I}(X, Y, Z)+R_{31} B_{z}^{I}(X, Y, Z), \\
& B_{y}(x, y, z)=R_{12} B_{x}^{I}(X, Y, Z)+R_{22} B_{y}^{I}(X, Y, Z)+R_{32} B_{z}^{I}(X, Y, Z),
\end{aligned}
$$




$$
B_{z}(x, y, z)=R_{13} B_{x}^{I}(X, Y, Z)+R_{23} B_{y}^{I}(X, Y, Z)+R_{33} B_{z}^{I}(X, Y, Z) .
$$

The rotation angles $(\alpha, \beta)$ are general, but since $\alpha$ is usually very small for tilt errors, the rotation matrix can be well approximated by letting $\cos (\alpha) \rightarrow 1$ and $\sin (\alpha) \rightarrow \alpha$. Defining the small tilt angles in the $x$ and $y$ directions:

$$
\theta_{x}=\alpha \cos (\beta), \quad \theta_{y}=\alpha \sin (\beta),
$$

the approximate matrix is then

$$
\left(R_{i j}\right) \approx\left(\begin{array}{ccc}
1 & 0 & -\theta_{x} \\
0 & 1 & -\theta_{y} \\
\theta_{x} & \theta_{y} & 1
\end{array}\right) .
$$

This is not quite orthogonal and leads to small violations of $\vec{\nabla} \cdot \vec{B}=0$. Keeping only terms that are linear in $\left(\theta_{x}, \theta_{y}, \Delta_{x}, \Delta_{y}, x, y\right)$, we have the approximate near-axis field:

$$
\begin{aligned}
& B_{x} \approx-\frac{1}{2} B_{0}^{\prime}\left(z-\Delta_{z}\right)\left[\left(x-\Delta_{x}\right)-\theta_{x}\left(z-\Delta_{z}\right)\right]+\theta_{x} B_{0}\left(z-\Delta_{z}\right), \\
& B_{y} \approx-\frac{1}{2} B_{0}^{\prime}\left(z-\Delta_{z}\right)\left[\left(y-\Delta_{x}\right)-\theta_{y}\left(z-\Delta_{z}\right)\right]+\theta_{y} B_{0}\left(z-\Delta_{z}\right), \\
& B_{z} \approx B_{0}\left(z-\Delta_{z}\right) .
\end{aligned}
$$

\section{Multiple Channels - Cross Talk}

We have treated a system of solenoids that are centered on a single straight axis except for individual misalignments and tilts. More complicated systems are of interest, in which solenoids are positioned along several axes that may have differing orientations. For example a multiple-beam linac could have parallel, closely packed channels, while a system of solenoids designed to bring multiple beams to a single focal spot would have several orientations. In such cases the problem of magnetic interference between channels (cross talk) is severe, and it is important to make good estimates of the unwanted field components. This does not mean that any significant cross talk is unacceptable. Generally the effects of unwanted fields can be greatly reduced by a symmetrical layout of channels and the addition of weak transverse dipoles and quadrupoles around each channel.

We need a simple formula for the external field produced by a solenoid with arbitrary position and orientation. This is provided by a generalization of eqn. (11.22) for the external global potential of a semi-infinite current layer: 


$$
\begin{aligned}
& J_{\theta}(r, z)=J_{\theta}(r) \Theta(z), \\
& S=\int_{0}^{\infty} d r J_{\theta}(r), \\
& \varphi \cong-\frac{\mu_{0} S}{2}\left\{\frac{\overline{r^{2}}}{2} \frac{1}{|\vec{r}|}+\frac{\overline{r^{4}}}{4}\left[\frac{-1}{2|\vec{r}|^{3}}+\frac{3 r^{2}}{4|\vec{r}|^{5}}\right]\right\} .
\end{aligned}
$$

Here we truncated after the lowest moments:

$$
S \overline{r^{2}}=\int_{0}^{\infty} d r r^{2} J_{\theta}, S \overline{r^{4}}=\int_{0}^{\infty} d r r^{4} J_{\theta}(r) .
$$

For a thin current layer with radius $\mathrm{R}$, we have $\overline{r^{2}}=R^{2}$ and $\overline{r^{4}}=R^{4}$. For a thick uniform current layer between $R_{1}$ and $R_{2}$ :

$$
\overline{r^{2}}=\frac{R_{2}^{3}-R_{1}^{3}}{3\left(R_{2}-R_{1}\right)}, \overline{r^{4}}=\frac{R_{2}^{5}-R_{1}^{5}}{5\left(R_{2}-R_{1}\right)} .
$$

Suppose a solenoid end, denoted by subscript i, is located at $\vec{r}_{i}$ and has direction; $\hat{e}_{i}$ then its external global potential $\left(\varphi_{i}\right)$ is obtained by substituting into eqn. (15.3) $|\vec{r}| \rightarrow\left|\vec{r}-\vec{r}_{i}\right|$ and

$$
r^{2}=|\vec{r}|^{2}-z^{2}=|\vec{r}|^{2}-\left(\vec{r} \cdot \hat{e}_{z}\right)^{2} \rightarrow\left|\vec{r}-\vec{r}_{i}\right|^{2}-\left(\vec{r} \cdot \hat{e}_{i}-\vec{r}_{i} \cdot \hat{e}_{i}\right)^{2}
$$

The quantities $S, \overline{r^{2}}$, and $\overline{r^{4}}$ are unchanged. Since $\varphi$ is a scalar quantity we have

$$
\varphi_{i} \cong-\frac{\mu_{0} S}{2}\left\{\frac{\overrightarrow{r^{2}}}{2} \frac{1}{\left|\vec{r}-\overrightarrow{r_{i}}\right|}+\frac{\overline{r^{4}}}{4}\left[\frac{-1}{2\left|\vec{r}-\vec{r}_{i}\right|^{3}}+\frac{3}{4} \frac{\left|\vec{r}-\vec{r}_{i}\right|^{2}-\left(\vec{r} \cdot \hat{e}_{i}-\vec{r}_{i} \cdot \hat{e}_{i}\right)^{2}}{\left|\vec{r}-\vec{r}_{i}\right|^{5}}\right]\right\} \text {. }
$$

Each magnet contributes two terms of this type. For example if the downstream end is at $\vec{r}_{2}$ and the current layer length is $\ell$, then the upstream end is at $\vec{r}_{1}=\vec{r}_{2}-\ell \hat{e}$. The overall minus sign in eqn. (15.7) is changed to a plus for the upstream magnet end. The total residual field is simply

$$
\vec{B}^{\text {residual }}=\vec{\nabla} \sum_{i} \varphi_{i},
$$


with applicable values of $\pm S, \vec{r}_{i}, \overline{r^{2}}$, and $\overline{r^{4}}$.

We have included only the two lowest-order terms in the expansion of $\varphi$; this should be sufficient for nearly any estimate. In fact the first (monopole) term,

$$
\varphi_{i} \approx-\frac{\mu_{0} S_{i}\left(\overline{r^{2}}\right)_{i}}{4\left|\vec{r}-\vec{r}_{i}\right|}
$$

should be a good rough guide. The field of the "monopole" term,

$$
\vec{B}_{i}^{\text {residual }}=\vec{\nabla} \varphi_{i} \approx \frac{\mu_{0} S_{i}\left(\overrightarrow{r^{2}}\right)_{i}\left(\vec{r}-\overrightarrow{r_{i}}\right)}{4\left|\vec{r}-\overrightarrow{r_{i}}\right|^{3}},
$$

is just a spherically symmetrical field with the same net flux as the magnet's direct field.

\section{Magnetic Materials Basics}

There are several reasons for placing highly permeable material outside solenoidal wire layers: confine return flux, shape flux surfaces, increase the bore field, and reduce total field energy. Another type of application is in the core of an induction module, however this involves time-dependent fields that do not have solenoidal symmetry and is therefore not considered here. A solenoid can also be a permanent magnet of low permeability without any wire layers.

\subsection{Ferromagnetism}

We are primarily interested in highly permeable ferromagnetic materials, i.e. those where the parallel alignment of atomic magnetic moments produces strong macroscopic fields in response to weak externally-applied fields. Ordinary paramagnetic materials are not of interest since their induced fields are very small, and ferromagnetic materials saturate at fields of only a few kilogauss or less. While the subject of magnetic materials is too vast (and beyond our competence) to even begin a summary here, a few remarks are made for background and clarity. For more detail see references [16-1, 2, 3].

At temperatures below their Curie points metallic Fe, Co, and Ni display spontaneous magnetization, in which the atomic magnetic moments line up in parallel to minimize the local energy density, i.e. they have the maximum value that can be projected in a particular direction. These atomic moments are smaller by a factor of several in the ferromagnetic material than in the isolated atoms. A ferromagnetic domain, in which the atomic moments are all essentially parallel, is usually only microns in diameter (but can be much larger in e.g. ferromagnetic tape), and a bulk material sample 
is usually made up of many small crystals, each containing many such domains. A macroscopic sample's total energy also includes contributions from small-scale fields around the domains as well as magnetostriction and domain wall structure. The small domain size and random orientation minimize the total energy in the absence of an applied field. These features can be viewed as the result of a competition between atomic and macroscopic (but small scale) forces. The domains resist reorientation, so even a very soft (easily magnetized) material can be in a persistent magnetized state of non-minimum energy in the absence of any external field. The three room-temperature ferromagnetic elements are often alloyed with each other and other elements such as $\mathrm{Cr}, \mathrm{Si}, \mathrm{Al}, \mathrm{Mn}, \mathrm{Cu}$, etc. to produce desired characteristics while retaining ferromagnetic features. A small amount of carbon is also usually present in iron as a separate phase mixed with the magnetic crystals.

Below $912^{\circ} \mathrm{C}$ the stable phase of pure iron is a body-centered-cubic crystal with Curie point $770^{\circ} \mathrm{C}$. The magnetic domains are usually aligned in the directions of the three cubic axes (say $\hat{e}_{x}, \hat{e}_{y}, \hat{e}_{z}$ ). This material is often referred to as "ferrite" - not to be confused with the ferromagnetic iron oxide ceramics of the same name that are used in rapidly pulsed cores. Nearly pure iron with about .1\% carbon has the combined properties of high permeability and high saturation field desired for application with high field solenoids. An example is 1010 steel $(\mathrm{Fe}, .08-.13 \% C, .3-.6 \% \mathrm{Mn})$, which is adopted for the linac module design presented in section 20.

Unlike the "ferritic steel" described above, beamline parts such as flanges and the beam pipe are not magnetic. This is achieved with "austenitic steel", which has facecentered-cubic structure, stabilized at normal temperatures by the addition of a large fraction of nickel. A common alloy of this type is 304 stainless steel $(\mathrm{Fe}, 18 \% \mathrm{Cr}, 8 \% \mathrm{Ni}$, $2 \% \mathrm{Mn}, .75 \% \mathrm{Si}, \mathrm{C} \leq .08 \%$ ), used for kitchenware as well as accelerator components.

When an externally produced field is applied to a permeable material, the magnetic domains tend to line up with it, again minimizing energy density. At low fields this happens by domain wall movement of favorably oriented domains at the expense of unfavorably oriented neighbors. For moderate-to-high external fields domains rotate into alignment. The process of domain growth by wall movement is resisted by internal fiction and occurs in tiny jumps with dissipation of energy as heat. For an excellent qualitative discussion of this process see reference [16-3].

Permanent magnets are manufactured from materials that lock in an externally induced magnetization along a preferred crystal axis (the easy axis) as they cool from a melt; examples are $\mathrm{SmCo}_{5}, \mathrm{Sm}_{2} \mathrm{Co}_{17}$, and $\mathrm{NdBFe}$. Amorphous ferromagnetic alloys such as metglass are produced as uncrystalized tape by very rapid cooling of a melt, and as such they have essentially zero anisotropy energy (along the tape). Although expensive, the insulated tape has ideal characteristics for transformers and induction cores pulsed on a $\mu$ s time scale. 


\subsection{Magnetization formalism}

If in a macroscopic region of a material the mean (vector) magnetic dipole moment per atom is $\vec{m}(\vec{r})$, and $n(\vec{r})$ is the atomic number density, then

$$
\vec{M}(\vec{r})=n(\vec{r}) \vec{m}(\vec{r})
$$

is called the magnetization density. For a highly permeable material this is an average over many domains. We expect $|\vec{m}|$ to be less than the maximum projected atomic moment, which is on the order of a Bohr magneton (Fe: $2.218 m_{B}$, Co: $1.714 m_{B}$, Ni: $\left..604 m_{B}\right)$, but this limit is approached at high applied fields. It was mentioned in section (2) that for solenoidal symmetry $\vec{M}$ has the form

$$
\vec{M}=M_{r}(r, z) \hat{e}_{r}+M_{z}(r, z) \hat{e}_{z},
$$

and that it provides a current density

$$
\vec{J}^{m a g}=\vec{\nabla} x \vec{M}=\hat{e}_{\theta}\left(\frac{\partial M_{r}}{\partial z}-\frac{\partial M_{z}}{\partial r}\right)
$$

The derivation of this relation is presented in many E\&M textbooks and is not repeated here. However, its plausibility is made clear by considering a cylinder of length $\ell$ and radius $R \ll \ell$, with uniform magnetization density $M \hat{e}_{z}$. Then eqn. (16.3) gives

$$
J_{\theta}^{m a g}=M \quad \delta(r-R),
$$

and the total circulating current is

$$
I^{m a g}=\ell M .
$$

We verify that this gives the correct net magnetic moment:

moment $=$ area $\mathrm{x}$ current $=\left(\pi R^{2}\right)(\ell M)=$ volume $\mathrm{x}\left(\begin{array}{l}\text { magnetization } \\ \text { density }\end{array}\right)=\sum_{\text {atoms }} m_{z}$.

Eqn. (2.1a) is now generalized to

$$
\vec{\nabla} x \vec{B}=\mu_{0}\left(\vec{J}+\vec{J}^{m a g}\right)=\mu_{0}(\vec{J}+\vec{\nabla} x \vec{M}),
$$

where $\vec{J}$ denotes the contribution from wire. It is convenient and conventional to define another field: 


$$
\vec{H} \equiv \frac{\vec{B}}{\mu_{0}}-\vec{M}
$$

so we write eqn. (16.5) as

$$
\vec{\nabla} \times \vec{H}=\vec{J}
$$

Eqn. (2.1b), $\vec{\nabla} \cdot \vec{B}=0$, is still true, so from eqn. (16.6) we have

$$
\vec{\nabla} \cdot \vec{H}=-\vec{\nabla} \cdot \vec{M}
$$

Therefore $\vec{H}$ may be regarded as being generated by the combination of wire current density $\vec{J}$ and the scalar quantity $-\vec{\nabla} \cdot \vec{M}$ (sometimes called the magnetic pole density). Applying a theorem of vector calculus [16-4], $\vec{H}$ may be derived from its curl and divergence according to

$$
\vec{H}=\vec{\nabla} x \int \frac{d^{3} r^{\prime}}{4 \pi} \frac{\overrightarrow{J^{\prime}}}{\left|\overrightarrow{r^{\prime}}-\vec{r}\right|}+\vec{\nabla} \int \frac{d^{3} r^{\prime}}{4 \pi} \frac{\overrightarrow{\nabla^{\prime}} \cdot \overrightarrow{M^{\prime}}}{\left|\overrightarrow{r^{\prime}}-\vec{r}\right|}
$$

assuming the sources vanish at large $|\vec{r}|$. It is useful to think of $\vec{H}$ as the part of $\vec{B}$ that is produced by distributed sources $\vec{J}$ and $\vec{\nabla} \cdot \vec{M}$, while $\mu_{0} \vec{M}$ itself is the local contribution to $\vec{B}$.

We have followed SI convention in defining $\vec{H}$, so in vacuum it differs from $\vec{B}$ by the factor $\mu_{0}^{-1}$. Unfortunately this causes $\vec{B}$ and $\vec{H}$ to have different units; these are Tesla or Webers $/ \mathrm{m}^{2}$ for $\vec{B}$ and Ampere/m for $\vec{H}$ and $\vec{M}$. If $|\vec{B}|$ is $1.0 \mathrm{~T}$ in vacuum then $|\vec{H}|$ is 795775 Ampere/m. Sometimes $\vec{H}$ is defined as $\vec{H}=\vec{B}-\mu_{0} \vec{M}$ instead of by eqn. (16.6) to avoid this inconvenience.

If Gaussian units are used then the factor $\mu_{0}$ in eqn. (16.6) is dropped and $\vec{B}, \vec{H}$, and $\vec{M}$ all have units of gauss $\left(1.0\right.$ gauss $\left.=10^{-4} \mathrm{~T}\right)$, but for $\vec{H}$ this unit is called the oersted. Also, for Gaussian units factors of $4 \pi$ and $\mathrm{c}$ are inserted as follows:

$$
\begin{aligned}
& \vec{J}^{\text {mag }}=c \vec{\nabla} x \vec{M}, \vec{H}=\vec{B}-4 \pi \vec{M}, \\
& \vec{\nabla} x \vec{H}=\frac{4 \pi}{c} \vec{J}, \quad \vec{\nabla} \cdot \vec{H}=-4 \pi \vec{\nabla} \cdot \vec{M}
\end{aligned}
$$


There is considerable variety in the names given for $\vec{B}$ and $\vec{H}$, although they tend to reflect their respective properties or uses. Some of these names are

$$
\begin{aligned}
& \vec{B} \text { : magnetic field, magnetic induction, magnetic flux density. } \\
& \vec{H} \text { : magnetic field, magnetic intensity, magnetization field. }
\end{aligned}
$$

\subsection{B-H relations and jump conditions}

So far we have not related $\vec{H}$ to $\vec{B}$ except by its definition, eqn. (16.6). In fact for a permeable ferromagnetic material there is no completely fixed relation except

$$
\left|\vec{B} / \mu_{0}-\vec{H}\right|=|\vec{M}|<M_{0}
$$

with $M_{0}$ the magnetization density within a single domain. For example, pure iron (natural isotopic mix) at 0 Kelvin has

$$
\rho=7.87 \times 10^{3} \mathrm{~kg} / \mathrm{m}^{3}, \text { atomic mass }=9.273 \times 10^{-26} \mathrm{~kg}, \mathrm{~m} / \mathrm{m}_{B}=2.218 .
$$

Inserting the natural constants

$$
m_{B}=9.274 \times 10^{-24} \mathrm{Am}^{2}, \mu_{0}=4 \pi \times 10^{-7} \mathrm{Tm} / \mathrm{A},
$$

we get

$$
M_{0}=\frac{\rho}{\operatorname{mass}} \frac{m}{m_{B}} m_{B}=1.746 \times 10^{6} \frac{A}{m}, \quad \mu_{0} M_{0}=2.194 T .
$$

Unalloyed iron with negligible carbon can approach this level of magnetization. An alloy of iron and cobalt $(70 \% \mathrm{Fe}, 30 \% \mathrm{Co})$ can increase $M_{0}$ by $10 \%$.

The value of $\vec{M}$ in a macroscopic sample actually depends on its history of exposure to externally generated fields as well as the instantaneous value of $\vec{H}$. By contrast a linear relation characterizes good permanent magnet blocks:

$$
\vec{B}=\hat{e}_{I I}\left(B^{r e m}+\mu_{0} \mu_{I I}^{r} H_{I I}\right)+\hat{e}_{\perp} \mu_{0} \mu_{\perp}^{r} H_{\perp},
$$

where $I I$ and $\perp$ refer to directions along and normal to the easy axis of magnetization. Here $B^{\text {rem }}$, called the remanent field, is simply the "permanent" value of $\mu_{0} M$ in a long sample, and the relative permeabilities $\mu_{I I}^{r}$ and $\mu_{\perp}^{r}$ are constants out to large values of $H$ and typically exceed unity by less than $10 \%$. For very large reverse $\mu_{0} H$ (depending on 
material but ideally of order $B^{\text {rem }}$ or greater) a block's properties become nonlinear and change irreversibly. Inside a permanent magnet block $H$ and $B$ are usually opposed.

It is apparent that the commonly assumed, linear, isotropic relation

$$
\vec{B}=\mu \vec{H}
$$

is of limited validity except for paramagnetic and diamagnetic substances at low fields (or $\mu_{0}$ vacuum). Nevertheless its use is sometimes a justifiable approximation. For example cast iron excited from an initial state of no remanent field exhibits such a linear permeability out to $|\vec{B}| \approx .3 T$, with $\mu \approx 400 \mu_{0}$. Some types of steel have $\mu$ greater than $10^{4} \mu_{0}$. Confusing the situation, eqn. (16.12) is often regarded as the definition of $\mu$ when $B$ and $H$ lie on the magnetization curve - see below. In fact we adopt this convention in sections 19 and 20, which deal with the Tosca ${ }^{\odot}$ model. If we assume the validity of eqn. (16.12) then the following definitions are made:

$$
K^{m} \equiv \mu^{r} \equiv \mu / \mu_{0}
$$

is the relative permeability, and

$$
\chi^{m}=K^{m}-1
$$

is the magnetic susceptibility. From the definition of $\vec{H}$ we then have

$$
\vec{M}=\frac{\vec{B}}{\mu_{0}}-\vec{H}=K^{m} \vec{H}-\vec{H}=\chi^{m} \vec{H}
$$

An improvement on eqn. (16.12), used in some computations, is

$$
\vec{B}=\vec{B}_{0}+\mu\left(\vec{H}-\vec{H}_{0}\right)
$$

where $\vec{B}_{0}$ and $\vec{H}_{0}$ are some particular values and $\mu$ is a local coefficient.

For many computations it is simply assumed that $\mu / \mu_{0} \rightarrow \infty$, i.e. $\vec{H}$ vanishes in the highly permeable material. However, to compute $\vec{B}$ inside the material requires specification of details about the permeability, even though it approaches infinity - see section 18 . 
If $\vec{B}$ and $\vec{H}$ are to be determined by solving their coupled partial differential equations, then jump conditions at vacuum-material interfaces are required. From eqns. (16.7) and (2.1b) these are

$$
\begin{aligned}
& H_{t} \text { is continuous, } \\
& B_{n} \text { is continuous, }
\end{aligned}
$$

where $t$ and $n$ refer to components tangential and normal to the interface.

These jump conditions suggest the utility of a highly permeable material for flux control or field shaping. Since $\mu_{0} \vec{H}$ can essentially vanish in the material, its tangential component on the vacuum side of the boundary also vanishes, as does the vacuum tangential component of $\vec{B}$. But the vacuum field can be derived from a scalar potential - see section $5, \vec{B}=-\mu_{0} \vec{\nabla} \phi$, which is single valued around the material if no wire is enclosed. Therefore $\phi$ is constant on the material surface. By analogy with electrostatics, the material acts like an electrical conductor, and strong normal fields may be restricted to particular locations where the surface is highly curved. Roughly speaking the field is channeled in and out of the material where desired, and routed away from locations (such as an induction core) where it could cause a problem. It is also apparent that stored energy might be reduced in this way since energy density in the material is roughly $B H / 2 \approx 0$, although the concentration of flux in particular locations outside the material tends to increase the local stored energy density. The design of the poles and return flux yoke of a cyclotron is an application of these ideas.

Field calculations for highly permeable materials are complicated by both the non-linearity of $B-H$ curves and the phenomenon of hysteresis. The latter is closely related to internal dissipation of energy during excitation and de-excitation. Following the textbooks, we imagine that in a macroscopic region of material $\vec{B}$ and $\vec{H}$ are parallel (more about this assumption below) and $H$ is increasing or decreasing slowly with time. In general, depending on the sign of $d H / d t$, two different curves are generated. If $H$ is cyclical, a closed loop in the $B-H$ plane is produced. If sufficiently large values of $H$ are in the cycle, so that the material is pushed well into saturation, then a maximal hysteresis loop is generated, and all other loops lie somewhere inside of it. The maximal hysteresis loop is the boundary of accessible points in the $B-H$ plane under the assumptions of parallelism and slow variation. This is considerably more restrictive than the condition (16.10). The intercepts of the maximal hysteresis curve with the $B$ and $H$ axes are called respectively the remanence $\left( \pm B_{\text {rem }}\right)$ and coercive force $\left( \pm H_{\text {coer }}\right)$. Some loops have zero bias current, i.e. no mean $H$, and form a nested set around the origin $(B=H=0)$. Other (biased) loops are offset from the origin and may or may not encircle it. In this picture we assume that the magnet is excited slowly enough that eddy currents are not appreciable and that magnetic domains have time to adjust their walls or orientations. All closed loops must cycle in a counter-clockwise direction to avoid a 
violation of the second law of thermodynamics. To see this, note that the differential work done by an external circuit is $H d B$ per unit volume. A clockwise loop would extract energy into the circuit while cooling the material (eliminating any need for controlled fusion energy). Through every point $(B, H)$ interior to the maximal loop we may draw a pair of paths, which are followed depending on whether $H$ is increasing or decreasing. A grid of crossing paths covers the accessible $B-H$ space, and any point may be reached by traversing several paths in their allowed directions. Complete demagnetization can be done by cycling $H$ with gradually decreasing amplitude.

\subsection{Magnetization curve}

Since ferromagnetic materials have a nonlinear magnetization response when driven over a large range, a generalization of eqn. (16.12) is required for computations. Usually it is assumed that a material is excited from an initially unmagnetized state $(B=H=0)$ and that $H$ only increases in this process, so there is no ambiguity from hysteresis. Then $B$ follows a known "magnetization curve", i.e. $B=F(H)$ - see the accompanying figure for examples (from [16-5]). Computations may be made by setting

$$
\vec{B}=\vec{H} F(\vec{r},|\vec{H}|) /|\vec{H}|,
$$

which assumes $\vec{B}$ and $\vec{H}$ remain parallel everywhere. The assumption of parallelism works if the response is linear, i.e. $F=\mu(\vec{r})|\vec{H}|$. To demonstrate this we write eqn. (16.9) in the form

$$
H_{i}=H_{i}^{J}+\int \frac{d^{3} r^{\prime}}{4 \pi} M_{j}^{\prime} \frac{\partial^{2}}{\partial x_{i} \partial x_{j}} \frac{1}{\left|\overrightarrow{r^{\prime}}-\vec{r}\right|}
$$

with subscripts $(i, j)$ denoting Cartesian components with a sum over $j$, and $\vec{H}^{J}$ is the part of $\vec{H}$ produced by currents in wire. For the linear isotropic response we have

$$
\vec{M}\left(\overrightarrow{r^{\prime}}\right)=\chi^{m}\left(\overrightarrow{r^{\prime}}\right) \vec{H}\left(\overrightarrow{r^{\prime}}\right),
$$

with $\chi^{m}=\mu / \mu_{o}-1$, and eqn. (16.19) can be reduced to a set of coupled linear equations by dividing the entire space into small volumes (finite element method). $\vec{H}$ is then obtained from $\vec{H}^{J}$ by a single matrix inversion, and the assumption of parallelism has lead to a unique solution. For a non-linear magnetization curve, we can use 


$$
\vec{M}=\frac{\vec{B}}{\mu_{0}}-\vec{H}=\left(\frac{F(\vec{r},|\vec{H}|)}{\mu_{0}|\vec{H}|}-1\right) \vec{H}
$$

and it is necessary to iterate to solve eqn. (16.19). Unfortunately it is not clear that this procedure leads to the physically correct solution, although this is generally assumed. Consider a system that is excited from the un-magnetized state by slowly increasing the wire current. If the material response is linear then $\vec{B}$ and $\vec{H}$ simply scale up proportional to current without changing direction. But the situation is changed when the material saturates and $\vec{B} \rightarrow \mu_{0} \vec{H}$; fields then approximate a vacuum pattern determined from the wire current alone, and in general they have a different direction than when the permeable material is effective. During the current increase $\vec{B}$ and $\vec{H}$ have changed direction; but this is different from the simple experimental conditions in which magnetization curves are determined. It is conceivable that for a gradually changing direction of $\vec{H}$, that $\vec{M}$ (and therefore $\vec{B}$ ) stays parallel, but this is an additional material property to be determined by experiment.

A mathematical model of hysteresis has been described in an interesting series of reports - see [16-6]. Recall that every $B-H$ point inside the limiting cycle is crossed by two curves with slopes $d B / d H$, depending on whether $H$ is increasing or decreasing. These slopes are found to be well approximated by the assumed formula

$$
\frac{d B}{d H}= \pm \alpha[f(H)-B]+g(H)
$$

where $\alpha$ is a constant and $(f, g)$ are material-dependent functions with examples given in the cited report. The choice $\pm \rightarrow+$ or - depends respectively on $H$ increasing or decreasing. If $\alpha, f$ and $g$ are known, then the magnetization curve $F(H)$ can be found in numerical form by integrating eqn. (16.22) from the origin $(B=H=0)$ with increasing $H$ :

$$
\begin{aligned}
& \frac{d B}{d H}+\alpha B=\alpha f(H)+g(H) \\
& F(H)=B=\int_{0}^{H} d H^{\prime}\left[\alpha f\left(H^{\prime}\right)+g\left(H^{\prime}\right)\right] e^{\alpha\left(H^{\prime}-H\right)} .
\end{aligned}
$$

For small field changes $(\Delta \mathrm{B}, \Delta \mathrm{H})$ the local energy density changes by $\mathrm{H} \Delta \mathrm{B}$. According to eqn. (16.22) this energy is produced irreversibly as heat $[\Delta Q= \pm \mathrm{H} \alpha(f(\mathrm{H})-\mathrm{B}) \Delta \mathrm{H}]$ and as a reversible increment $[\Delta U=\mathrm{H} g(\mathrm{H}) \Delta \mathrm{H}]$. In this model we are therefore able to identify a stored "field energy" that depends only on $\mathrm{H}$ : 


$$
U^{\text {field }}=\int_{0}^{\mathrm{H}} d \mathrm{H}^{\prime} \quad \mathrm{H}^{\prime} \quad g\left(\mathrm{H}^{\prime}\right) .
$$

For the simple linear relation $g=\mu=\mathrm{B} / \mathrm{H}$ this becomes $U^{\text {field }}=\mu \mathrm{H}^{2} / 2$.

\subsection{Permanent magnet solenoid}

Finally we consider a simple permanent magnet solenoid consisting of an annulus of constant magnetization $M \hat{e}_{z}$ between radii $R_{1}$ and $R_{2}$, with length $\ell$, and centered at $z=0$. The magnetization currents are simply a pair of thin layers:

$$
J_{\theta}^{\mathrm{mag}}=-\frac{\partial M_{z}}{\partial r}=M\left[\delta\left(r-R_{2}\right)-\delta\left(r-R_{1}\right)\right] .
$$

Applying the thin layer result, eqn. (4.6), we have the on-axis field,

$$
B_{0}(z)=\frac{\mu_{0} M}{2}\left[\begin{array}{l}
\frac{(z+\ell / 2)}{\sqrt{(z+\ell / 2)^{2}+R_{2}^{2}}}-\frac{(z-\ell / 2)}{\sqrt{(z-\ell / 2)^{2}+R_{2}^{2}}} \\
-\frac{(z+\ell / 2)}{\sqrt{(z+\ell / 2)^{2}+R_{1}^{2}}}+\frac{(z-\ell / 2)}{\sqrt{(z-\ell / 2)^{2}+R_{1}^{2}}}
\end{array}\right] .
$$

This function is plotted below for $\left(\mu_{0} M=1.0 \mathrm{~T}, R_{2}=.5 \mathrm{~m}, R_{1}=.25 \mathrm{~m}, \quad \ell=1.0 \mathrm{~m}\right)$. Note that $B_{0}$ is negative in the solenoid bore and positive outside, and its maximum absolute value is only $.19 \mu_{0} M$. When such a solenoid is used as a particle beam lens, its focal strength is proportional to the integral of $B_{0}^{2}$, so this is not a very effective use of material, although such lenses have been used to confine electron beams in Klystrons.

It can be easily verified that

$$
\int_{-\infty}^{+\infty} d z B_{0}(z)=0
$$

In fact this relation is true for any solenoid made only of magnetic materials since $\vec{\nabla} x \vec{H}=\vec{J}$ requires that the line integral of $\vec{H}$ around any closed loop must equal the enclosed wire current:

$$
\oint \overrightarrow{d r} \cdot \vec{H}=I=0 .
$$

In the present case we may take the integration loop along the entire axis and return at large radius, where there is a vanishing contribution. The line integral of $\overrightarrow{\mathrm{B}} / \mu_{0}$ along any closed path in vacuum equals only the enclosed wire current since $\vec{M}$ vanishes on the 
path. This is a special feature of $\vec{J}^{m a g}=\vec{\nabla} x \vec{M}$ with $\vec{M}$ non-zero only in a bounded region of space.

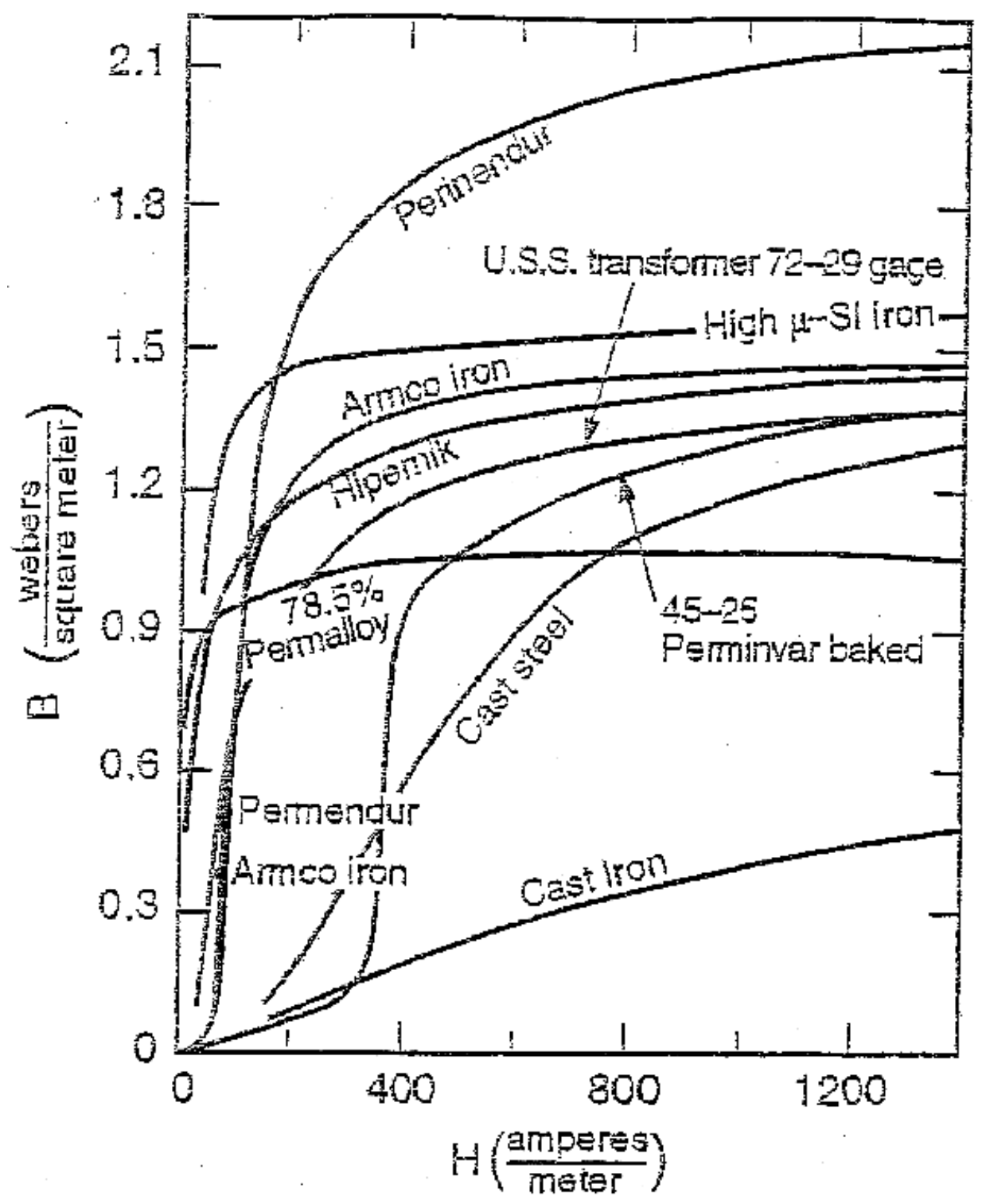

$\mathrm{mu} 0 \mathrm{M}=1.0$;

$\mathrm{R} 2=.5$;

$\mathrm{R} 1=.25$;

$1=1.0$;

$$
\begin{aligned}
\mathrm{B} 0\left[\mathrm{z}_{3}\right]= & \mathrm{mu} 0 \mathrm{M} / \\
2^{*}\left((\mathrm{z}+1 / 2) /\left((\mathrm{z}+1 / 2)^{\wedge} 2+\mathrm{R} 2^{\wedge} 2\right)^{\wedge} .5-(\mathrm{z}-\right. & 1 / 2) /\left((\mathrm{z}-1 / 2)^{\wedge} 2+\mathrm{R} 2^{\wedge} 2\right)^{\wedge} .5-(\mathrm{z}+ \\
& 1 / 2) /\left((\mathrm{z}+1 / 2)^{\wedge} 2+\mathrm{R} 1^{\wedge} 2\right)^{\wedge} .5+(\mathrm{z}- \\
& \left.1 / 2) /\left((\mathrm{z}-1 / 2)^{\wedge} 2+\mathrm{R} 1^{\wedge} 2\right)^{\wedge} .5\right)
\end{aligned}
$$


$\operatorname{Plot}[\mathrm{B} 0[\mathrm{z}],\{\mathrm{z},-2,2\}]$;

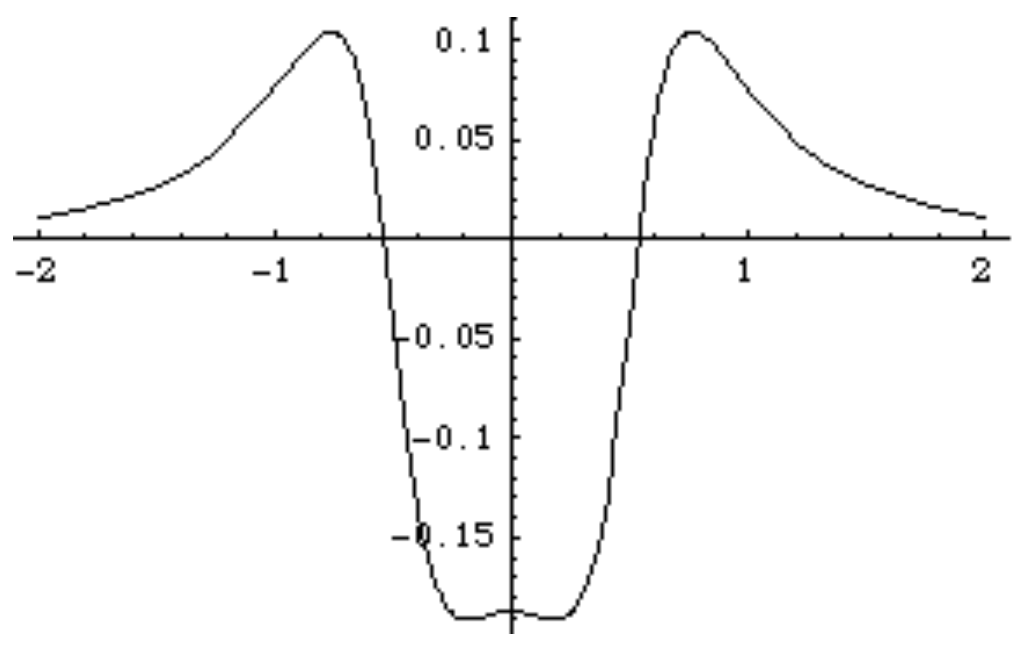

\section{References}

16-1 Bozorth, R.M., Ferromagnetism, IEEE Press (1993).

16-2 Kittel, C., Introduction to Solid State Physics, $7^{\text {th }}$ ed., ch. 15, John Wiley \& Sons (1996).

16-3 Feynman, R., R. Leighton, and M. Sands, The Feynman Lectures on Physics, Vol. II, ch. 37, Addison - Wesley, Reading, MA (1964).

16-4 Panofsky, W.K.H., and M. Phillips, Classical Electricity and Magnetism, $2^{\text {nd }}$ ed., pp. 2-5, Addison - Wesley, Reading, MA (1962).

16-5 Chao, A., and M. Tigner, Handbook of Accelerator Physics and Engineering, World Scientific (1999).

16-6 Hodgdon, M., Applications of a Theory of Ferromagnetic Hysteresis, IEEE Transactions on Magnetics, Vol. 24, No 1.

\section{Wire Layer Embedded in a Highly Permeable Yoke}

A simple geometry that displays flux control is a wire layer (of any shape) that has net azimuthal current $I$ and is embedded in a long permeable annulus (yoke) with $\mu=\infty$. The inner radius of the annulus/yoke is at $r=b$, which is also the magnet's bore radius, and a narrow slot connects the wire layer to the bore at $z=0$ (see figure). 


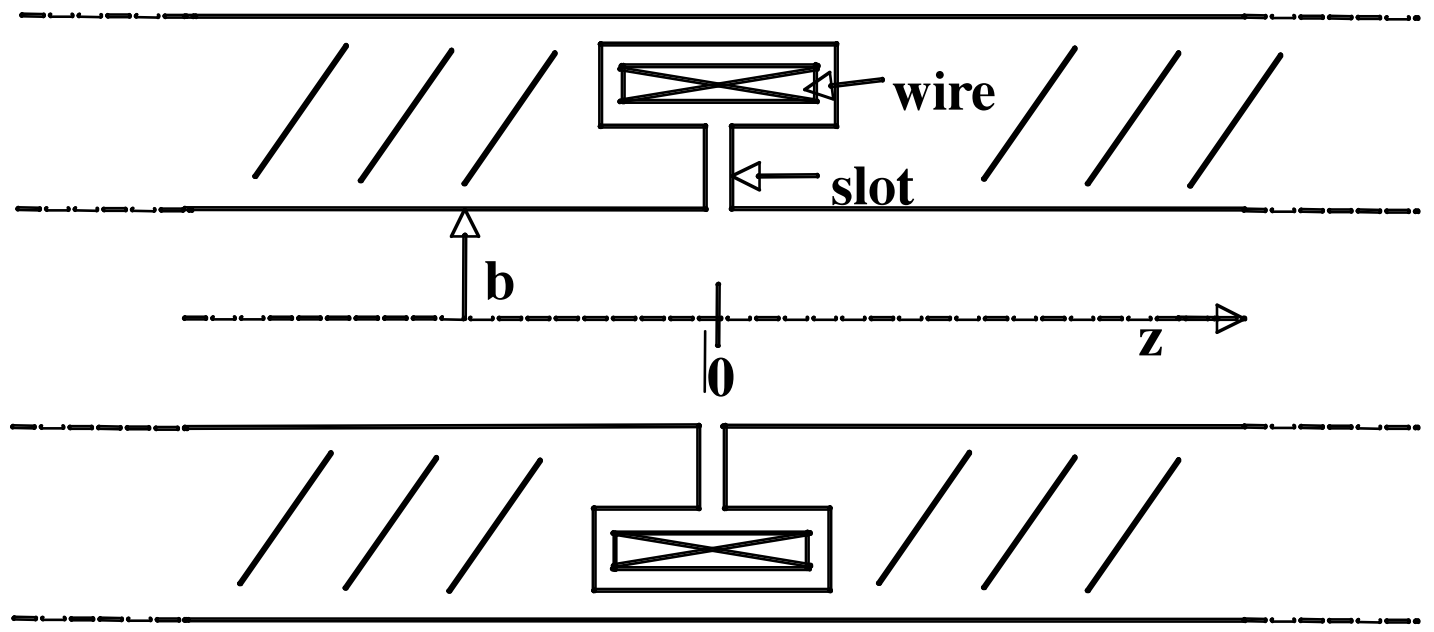

Inside the bore, where

$$
\vec{H}=\frac{\vec{B}}{\mu_{0}}=-\overrightarrow{\nabla \phi},
$$

we have

$$
\nabla^{2} \phi=0, \quad \nabla^{2} B_{z}=0
$$

Along the inner surface of the annulus $\phi$ is constant and $B_{z}$ vanishes, except at $z=0$, where $\phi$ jumps and $B_{z}$ is infinite:

$$
\begin{aligned}
& \phi(b, z)= \begin{cases}+I / 2 & z<0 \\
-I / 2 & z>0,\end{cases} \\
& B_{z}(b, z)=\mu_{0} I \delta(z) .
\end{aligned}
$$

Here the yoke is assumed to extend to $z= \pm \infty$ for computations. Outside the yoke $\phi$ is constant except for the intrinsic discontinuity described in section 5; it runs from any point on the exterior of the yoke to $r=\infty$.

To solve for $B_{z}$ in the magnet bore we apply a Fourier integral transform to eqn. (17.2b): 


$$
\begin{aligned}
& \tilde{B}_{z}(r, k)=\int_{-\infty}^{+\infty} d z \quad e^{-i k z} B_{z}(r, z), \\
& \frac{1}{r} \frac{\partial}{\partial r} r \frac{\partial \tilde{B}_{z}}{\partial r}-k^{2} \tilde{B}_{z}=0 .
\end{aligned}
$$

Equation (17.6) is satisfied by

$$
\tilde{B}_{z}=f(k) I_{0}(k \mid r),
$$

where $I_{0}$ is the modified Bessel function and $f(k)$ is to be determined from the boundary condition (17.4). Since we may write the delta function as

$$
\delta(z)=\int_{-\infty}^{+\infty} d k \frac{e^{i k z}}{2 \pi}
$$

we have immediately from equations (17.4) and (17.7)

$$
B_{z}=\int_{-\infty}^{+\infty} d k \frac{e^{i k z}}{2 \pi} \tilde{B}_{z}=\mu_{0} I \int_{-\infty}^{+\infty} d k \frac{e^{i k z}}{2 \pi} \frac{I_{0}(k \mid r)}{I_{0}(k \mid b)} .
$$

A convenient form for computations, which displays scaling with $r / b$ and $z / b$ is

$$
B_{z}=\frac{\mu_{0} I}{\pi b} \int_{0}^{\infty} d y \frac{\cos (y z / b) I_{0}(y r / b)}{I_{0}(y)}
$$

where $y \equiv k b$. The radial field is obtained from eqn. (17.9) by integrating $\vec{\nabla} \cdot \vec{B}=0$ :

$$
\begin{gathered}
B_{r}=-\mu_{0} I \int_{-\infty}^{+\infty} d k \frac{k}{|k|} i \frac{e^{i k z}}{2 \pi} \frac{I_{1}(k \mid r)}{I_{0}(k \mid b)} \\
=\frac{\mu_{0} I}{\pi b} \int_{0}^{\infty} d y \sin (y z / b) \frac{I_{1}(y r / b)}{I_{0}(y)} .
\end{gathered}
$$

The bore field is displayed for several values of $r / b$ at the end of this section. A strong (exponential) falloff with $|z| / b$ is apparent and contrasts sharply with the $|z|^{-3}$ fall off from a lens without a yoke. This calculation is formally equivalent to that of an electrostatic field in a conducting pipe that is split at $z=0$ and with a potential difference applied between the two sides.

The potential $\phi$ can be derived from eqn. (17.9) by integrating in $z$, however at this point it is more instructive to use an expansion in ordinary Bessel functions: 


$$
-\phi=\frac{I}{2}+\sum_{i=1}^{\infty} A_{i} J_{0}\left(x_{i} r / b\right) e^{-x_{i} z / b},
$$

valid for $z>0$. For $z<0, \phi$ is determined by its antisymmetry:

$$
\phi(r,-z)=-\phi(r, z)
$$

so the there is exponential decay in both directions. The $x_{i}$ are the zeros of $J_{0}$, and the coefficients $A_{i}$ are determined from the condition

$$
\begin{aligned}
& 0=-\phi(r, 0)=\frac{I}{2}+\sum_{i=1}^{\infty} A_{i} J_{0}\left(x_{i} r / b\right) \\
& A_{i}=-\frac{I}{x_{i} J_{1}\left(x_{i}\right)} .
\end{aligned}
$$

We have for $z>0$,

$$
\begin{aligned}
& -\phi=\frac{I}{2}-I \sum_{i=1}^{\infty} \frac{J_{0}\left(x_{i} r / b\right) e^{-x_{i} z / b}}{x_{i} J_{1}\left(x_{i}\right)}, \\
& B_{z}=-\mu_{0} \frac{\partial \phi}{\partial z}=\mu_{0} \frac{I}{b} \sum_{i=1}^{\infty} \frac{J_{0}\left(x_{i} r / b\right) e^{-x_{i} z / b}}{J_{1}\left(x_{i}\right)} .
\end{aligned}
$$

The first five values of $x_{i}$ and $J_{1}\left(x_{i}\right)$ are tabulated to aid in computations:

\begin{tabular}{|c|c|c|}
\hline$i$ & $x_{i}$ & $J_{1}\left(x_{i}\right)$ \\
\hline 1 & 2.4048255577 & .5191474973 \\
2 & 5.5200781103 & -.3402648066 \\
3 & 8.6537279129 & .2714522999 \\
4 & 11.7915344390 & -.2324598314 \\
5 & 14.9309177085 & .2065464331 \\
\hline
\end{tabular}

The on-axis field can be calculated from either the Fourier integral or Bessel series, although the latter is poorly convergent for $|z| / b \approx 0$. The leading term of the series is a good approximation for $|z| / b \geq 1$ : 


$$
B_{0}(z) \rightarrow \frac{\mu_{0} I}{b} \frac{e^{-x_{1} \mid z / b}}{J_{1}\left(x_{1}\right)}
$$

but is large by a factor of 2.9 at $z=0$. An excellent approximation for all $z$ is

$$
B_{0}(z) \approx \frac{\mu_{0} I}{b} \frac{1}{J_{1}\left(x_{1}\right)} \frac{1}{\left[2 \cosh \left(x_{1}|z| / s b\right)\right]^{s}},
$$

with $s=1.538781$. This has maximum relative error of $\pm 2 \times 10^{-4}$ at $|z| / b=(0, \pm .9 b)$.

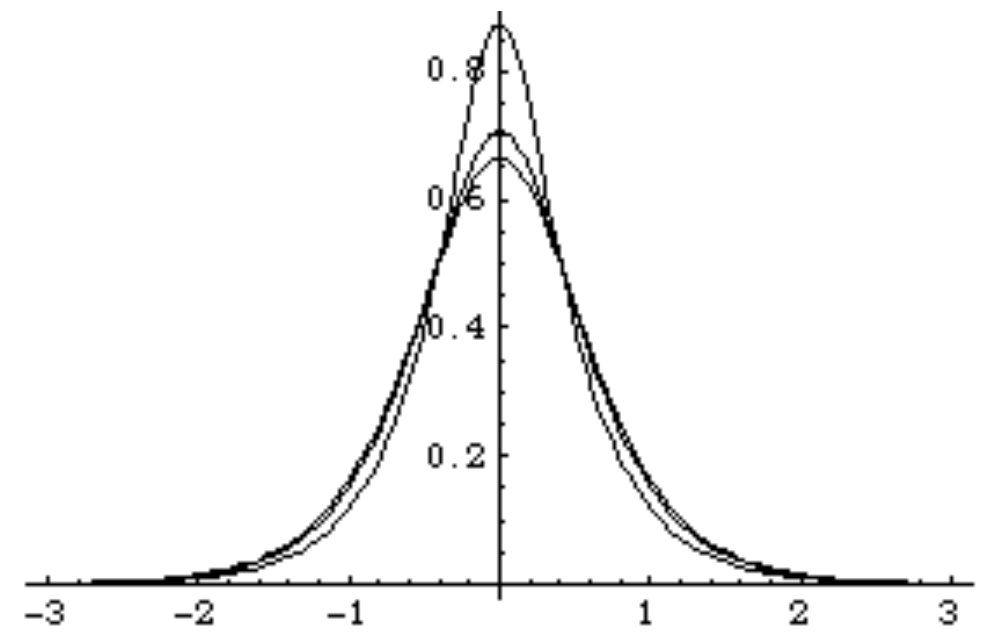

$B_{z}(r, z)$ for $r=(0, b / 4, b / 2)$. In this case $\mu_{0} I=1.0 T m$ and $b=1.0 m$.

\section{Periodic Thin Current Laver with a Highly Permeable Yoke}

The purpose of this section is only to present some considerations of return flux controls; for a practical example see section 20.

Return flux can be channeled by a permeable annulus if the field there is kept below saturation $\left(B_{\text {sat }} \approx 1.0-2.0 \mathrm{~T}\right.$ for magnet iron). For a periodic system of solenoids having the same polarity, the field in the annulus or "yoke" is roughly

$$
B_{\text {yoke }} \approx-\frac{R^{2} B_{\text {bore }} \ell / P}{R_{2}^{2}-R_{1}^{2}},
$$

where $R$ is the wire radius, $R_{1}$ and $R_{2}$ are the yoke radii, $\ell / P$ is the ratio of wire layer length to period length, and $B_{\text {bore }}$ is the peak bore field. This assumes no flux returns at $r=\alpha$. The volume of the yoke should be at least $B_{\text {bore }} / B_{\text {sat }}$ times the volume of magnet 
bore to prevent stray flux from leaking into the outside world. In practice there will be gaps in the yoke, so some flux lines will bulge out anyway.

The field in the presence of a highly permeable yoke can be examined with the simple periodic model:

$$
\begin{aligned}
& J_{\theta}= \begin{cases}S \delta(r-R) & -\ell / 2<z<\ell / 2, \\
0 & \ell / 2<|z|<P / 2,\end{cases} \\
& \mu= \begin{cases}\infty & R_{1}<r<R_{2}, \\
0 & \text { other } r,\end{cases}
\end{aligned}
$$

with $R<R_{1}$ and $J_{\theta}$ repeating with period P. Note that there are no gaps in the yoke model. The Fourier expansion of the current density is

$$
J_{\theta}=\delta(r-R)\left[\frac{S \ell}{P}+\sum_{n=1}^{\infty} \delta_{n} \cos \left(k_{n} z\right)\right] \equiv \delta(r-R) S(z)
$$

with

$$
\delta_{n}=\frac{2 S}{\pi n} \sin \left(\frac{\pi n \ell}{P}\right), k_{n}=\frac{2 \pi n}{P} .
$$

In the yoke $\vec{H}=\vec{B} / \mu \rightarrow 0$ by assumption, and since the tangential components of $\vec{H}$ at $R_{1}$ and $R_{2}$ are continuous, we conclude that $B_{z}=\mu_{0} H_{z}=0$ at $r=R_{1-}$ and $r=R_{2+}$. Since $\vec{B}$ must vanish as $r \rightarrow \infty$, it must also vanish for all $r>R_{2}$. For $r<R_{1}$ we must simply solve

$$
\nabla^{2} B_{z}=-\mu_{0} \frac{1}{r} \frac{\partial}{\partial r} r J_{\theta}
$$

with the boundary condition $B_{z}\left(R_{1-}\right)=0$.

The value of $\vec{B}$ inside the yoke is indeterminate unless more information is given about the permeability, which must be assumed to be not quite infinite. For example, suppose we have a non-linear magnetization curve

$$
\vec{B}=\frac{\vec{H}}{|\vec{H}|} \mathrm{F}(|\vec{H}|)
$$




$$
\vec{H}=\frac{\vec{B}}{|\vec{B}|} \mathrm{F}^{-1}(|\vec{B}|)
$$

Then in the yoke we must solve $\vec{\nabla} \cdot \vec{B}=0$ along with

$$
\vec{\nabla} x\left[\frac{\vec{B}}{|\vec{B}|} \mathrm{F}^{-1}(|\vec{B}|)\right]=\vec{\nabla} x \vec{H}=0
$$

The boundary conditions for the yoke interior are that $B_{r}$ be continuously joined to its vacuum values at $R_{1}$ and $R_{2}$ and that the net flux of the system vanish. For the simple assumption that $\mu$ is very large but constant, the Cartesian components of the yoke field satisfy

$$
\nabla^{2} \vec{B}=\vec{\nabla} \vec{\nabla} \cdot \vec{B}-\vec{\nabla} x(\vec{\nabla} x \vec{B})=-\vec{\nabla} x(\vec{\nabla} x \mu \vec{H})=0
$$

Returning to the analytical model and assuming $B / H$ is infinite but constant in the yoke; we have inside all four radial zones

$$
\nabla^{2} B_{z}=0
$$

At zone boundaries $\left(R, R_{1}, R_{2}\right)$ both $B_{r}$ and $\partial B_{z} / \partial r$ are continuous, while $B_{z}$ jumps across the wire:

$$
B_{z}\left(R_{+}, z\right)-B_{z}\left(R_{-}, z\right)=-\mu_{0} S(z)
$$

At the yoke boundaries $B_{z}$ jumps from zero in vacuum to a finite value in the material, which is found by solving the model equations. We proceed by solving for the field produced by individual terms of the expansion [eqn. (18.4)] for $J_{\theta}$. First, the term $S \ell / P$ produces

$$
B_{z} \rightarrow\left\{\begin{array}{ccc}
\frac{\mu_{0} S \ell}{P} & & 0<r<R, \\
0 & R<r<R_{1}, \\
-\frac{R^{2}}{R_{2}^{2}-R_{1}^{2}} \frac{\mu_{0} S \ell}{P} & R_{1}<r<R_{2}, \\
0 & R_{2}<r<\infty .
\end{array}\right.
$$

This function has net zero flux, vanishes at $r=\infty$, and has no on-axis contribution from the permeable material. Next consider the field from any periodic term of eqn. (18.4); this has the form 


$$
\begin{aligned}
& S(z) \rightarrow \delta_{n} \cos \left(k_{n} z\right), \\
& B_{z} \rightarrow \mu_{0} f_{n}(r) \delta_{n} \cos \left(k_{n} z\right), \\
& B_{r} \rightarrow \mu_{0} g_{n}(r) \delta_{n} \sin \left(k_{n} z\right) .
\end{aligned}
$$

Inside each zone $f_{n}$ satisfies

$$
\frac{1}{r} \frac{d}{d r} r \frac{d f_{n}}{d r}-k_{n}^{2} f_{n}=0
$$

and from $\vec{\nabla} \times \vec{B}=0$, inside each zone

$$
g_{n}=\frac{1}{k_{n}} \frac{\partial f_{n}}{\partial r}
$$

Equation (18.14) is a modified Bessel equation, so $f_{n}(r)$ is a linear combination of $I_{0}\left(k_{n} r\right)$ and $K_{0}\left(k_{n} r\right)$ in every zone, and $g_{n}(r)$ is the combination of $I_{1}\left(k_{n} r\right)$ and $K_{1}\left(k_{n} r\right)$ determined from eqn. (18.15). Applying the various boundary and jump conditions and finiteness at $r=0$ we find, suppressing the subscript $(n)$ :

$$
\begin{aligned}
\frac{0<r<R}{f(r)} & =A I_{0}(k r), \\
g(r) & =A I_{1}(k r), \\
\frac{R<r<R_{1}}{f(r)} & =B\left[\frac{I_{0}(k r)}{I_{0}\left(k R_{1}\right)}-\frac{K_{0}(k r)}{K_{0}\left(k R_{1}\right)}\right], \\
g(r) & =B\left[\frac{I_{1}(k r)}{I_{0}\left(k R_{1}\right)}+\frac{K_{1}(k r)}{K_{0}\left(k R_{1}\right)}\right], \\
\frac{R_{1}<r<R_{2}}{f(r)} & =C\left[\frac{I_{0}(k r)}{I_{1}\left(k R_{2}\right)}+\frac{K_{0}(k r)}{K_{1}\left(k R_{2}\right)}\right],
\end{aligned}
$$




$$
\begin{aligned}
g(r) & =C\left[\frac{I_{1}(k r)}{I_{1}\left(k R_{2}\right)}-\frac{K_{1}(k r)}{K_{1}\left(k R_{2}\right)}\right], \\
\frac{R_{2}<r<\infty}{f(r)} & =g(r)=0,
\end{aligned}
$$

with coefficients

$$
\begin{aligned}
& A=\frac{k R}{I_{0}\left(k R_{1}\right)}\left[I_{1}(k R) K_{0}\left(k R_{1}\right)+K_{1}(k R) I_{0}\left(k R_{1}\right)\right], \\
& B=k R I_{1}(k R) K_{0}\left(k R_{1}\right), \\
& C=\frac{R I_{1}(k R)}{R_{1} I_{0}\left(k R_{1}\right)}\left[\frac{I_{1}\left(k R_{1}\right)}{I_{1}\left(k R_{2}\right)}-\frac{K_{1}\left(k R_{1}\right)}{K_{1}\left(k R_{2}\right)}\right]^{-1} .
\end{aligned}
$$

We have used the Bessel function identity,

$$
I_{1}(x) K_{0}(x)+I_{0}(x) K_{1}(x)=\frac{1}{x},
$$

to simplify these expressions.

Plots. $B_{z}$ vs. $z$ for various $r$ are given below for the case $\left(R=.2, R_{1}=.6, R_{2}=.8, P=1.0, \ell=.8, \mu_{0} S \ell / P=10.0 T\right)$. The suppression of the external field in the zone $R<r<R_{1}$ is evident - compare section (10). A (very expensive) total of 200 terms were summed.

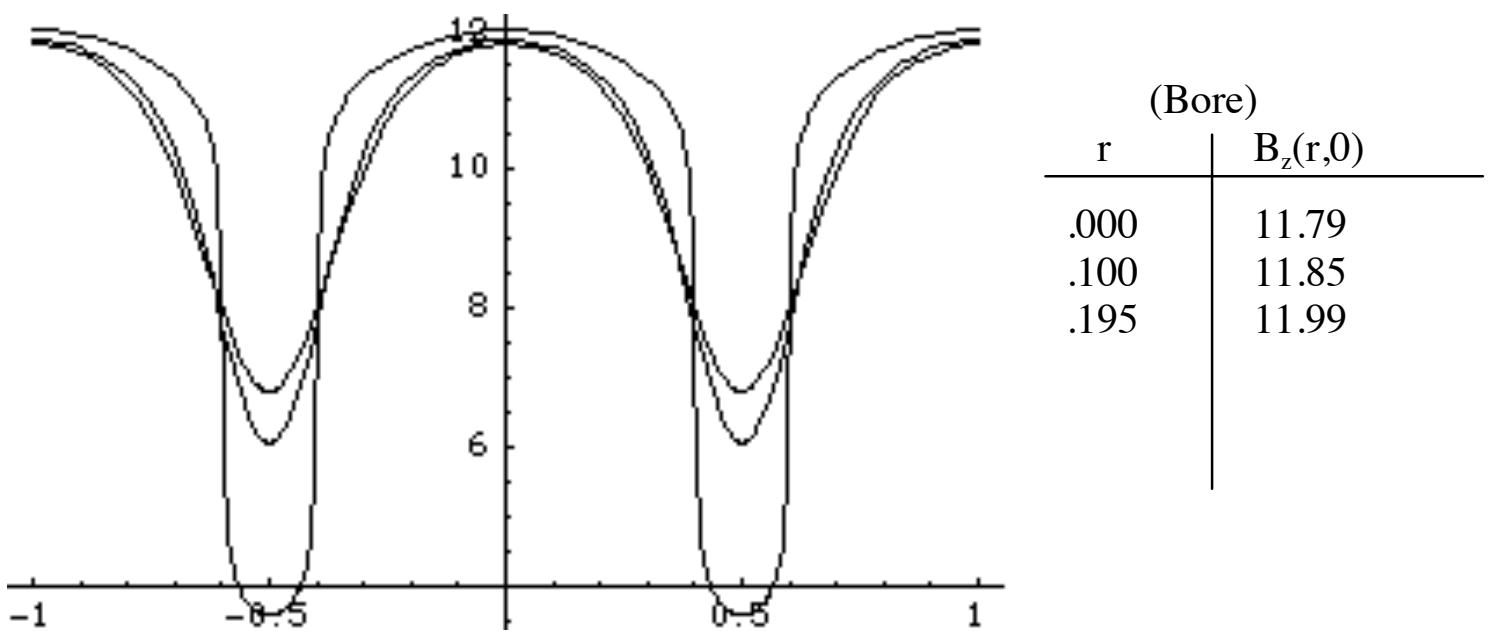



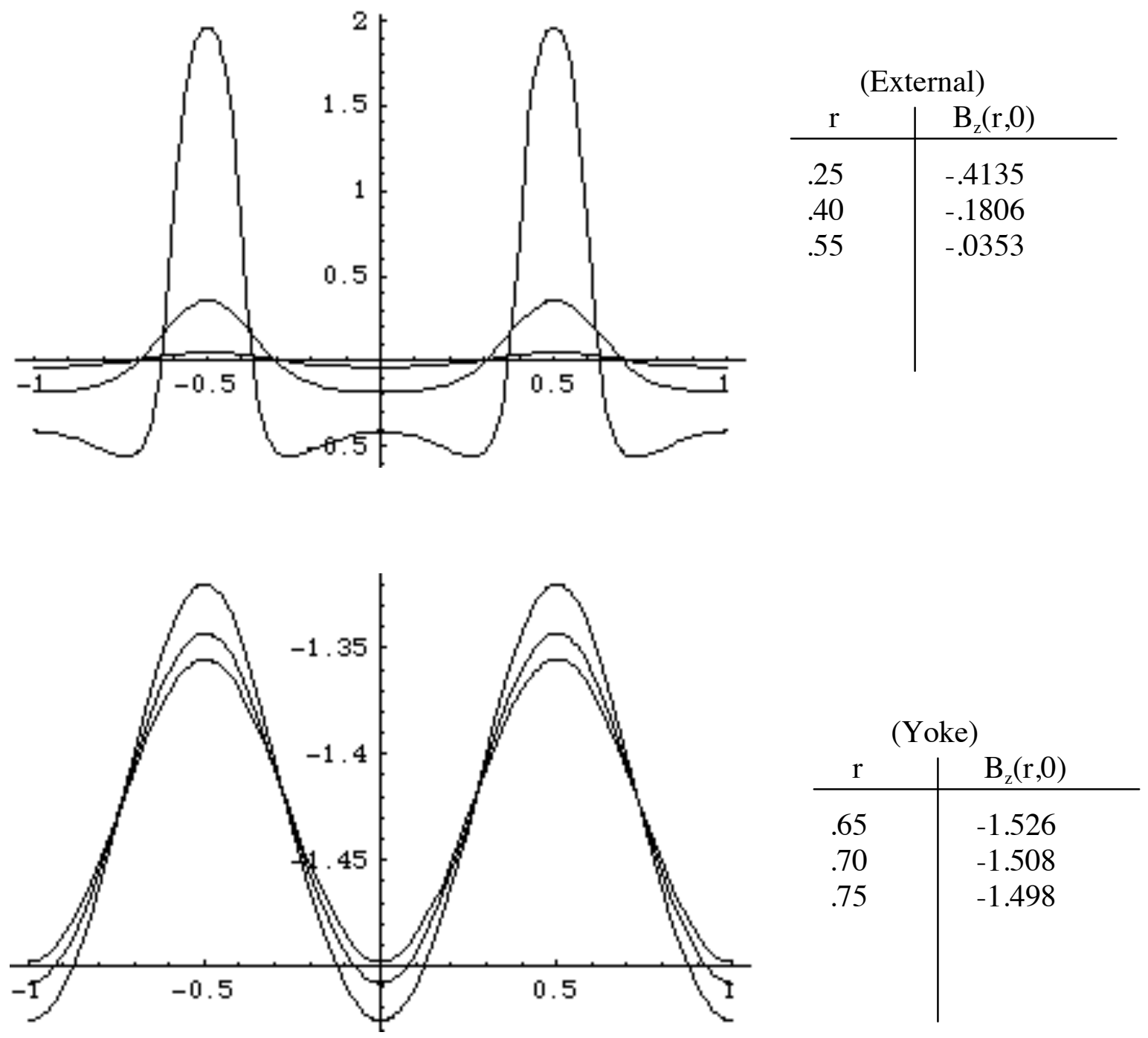

\section{Tosca $^{\circledR}$ Model}

Many computer programs have been written for the purpose of solving Laplace's or Poisson's equation under given boundary conditions. A number of numerical methods of solution are available for solving a system of partial differential equations; one of the most commonly used is finite element discretization. Finite element analysis (FEA) requires special enhancements, which are described briefly in this chapter, to make it applicable to electromagnetic field calculations.

The computer programs for magnetic fields most widely used in the accelerator engineering community are the public domain code POISSON [1-7] and the commercial code Tosca $^{(}$[1-7]. POISSON can simulate only 2D planar or axisymmetric geometries, whereas Tosca ${ }^{\odot}$ allows calculations in 2D and 3D. Since today's personal computers have become sufficiently powerful, the fidelity of these codes is so good that often simulations 
are used to check measurements, and large magnetic devices are designed without the need for building prototypes. For instance, a field accuracy of the order of parts in $10^{6}$ is required for simulating shielded superconducting NMR systems. Another application is to cyclotron magnets with their stringent field accuracies (parts in $10^{5}$ ), which can nowadays be entirely designed using $\operatorname{Tosca}^{\circledR}$ alone.

FEA codes are especially well suited for modeling magnetic fields with nonlinear materials present, e.g. magnet steel or permanent magnets. Forces on conductors and pole pieces can be easily calculated. In addition, harmonic or transient field problems with eddy currents present can be solved. Eddy current heating as well as magnetic forces can be coupled to thermal and structural FEA simulations for the engineering design.

\subsection{General solution strategy in 3D}

In this section the notation for fields differs somewhat from that used in the rest of the report in order to correspond to Tosca ${ }^{\circledR}$ conventions. A detailed description of how to numerically solve Maxwell's equations is beyond the scope of this report. Nevertheless, since the computation of magnetic fields requires a few special numerical techniques, the peculiarities are briefly mapped out here. We want to discretize Maxwell's equations:

$$
\begin{aligned}
& \vec{\nabla} x \vec{E}=-\frac{\partial \vec{B}}{\partial t}, \\
& \vec{\nabla} x \vec{H}=\frac{\partial \vec{D}}{\partial t}+\vec{j}, \\
& \vec{\nabla} \cdot \vec{D}=\rho, \\
& \vec{\nabla} \cdot \vec{B}=0,
\end{aligned}
$$

with $\vec{D}=\varepsilon \vec{E}, \vec{B}=\mu \vec{H}$, and $\vec{j}=\sigma \vec{E}$ (or specified). In these equations $\vec{E}$ and $\vec{H}$ are the electric and magnetic fields and $\vec{D}$ and $\vec{B}$ are the electric and magnetic flux densities. The current density is denoted by $\vec{j}$, and $\rho$ denotes the charge density. The three material functions $\varepsilon, \mu$, and $\sigma$ (permittivity, permeability, and conductivity) are in general functions of the spatial coordinates and also nonlinear functions of the electromagnetic field strength, e.g. the magnetization curve in metal. All three material properties may be tensors, as in laminated transformer cores and permanent magnets.

The usual procedure for solving Maxwell's equations is to simplify them as far as possible, first setting some of the quantities $\varepsilon, \mu$, or $\sigma$ equal to constants if possible, and then deducing a second order differential equation. This second-order differential equation for some intermediate function (typically a vector potential, scalar potential, or both) can then be discretized and numerically solved for realistically shaped structures by means of finite difference, finite element, or integral methods. The finite element method is based on division of the domain of these equations (volume of space in which the 
equations are satisfied) into small volumes (the finite elements) as shown in Figure 1. Within each finite element a simple polynomial is used to approximate the solution.

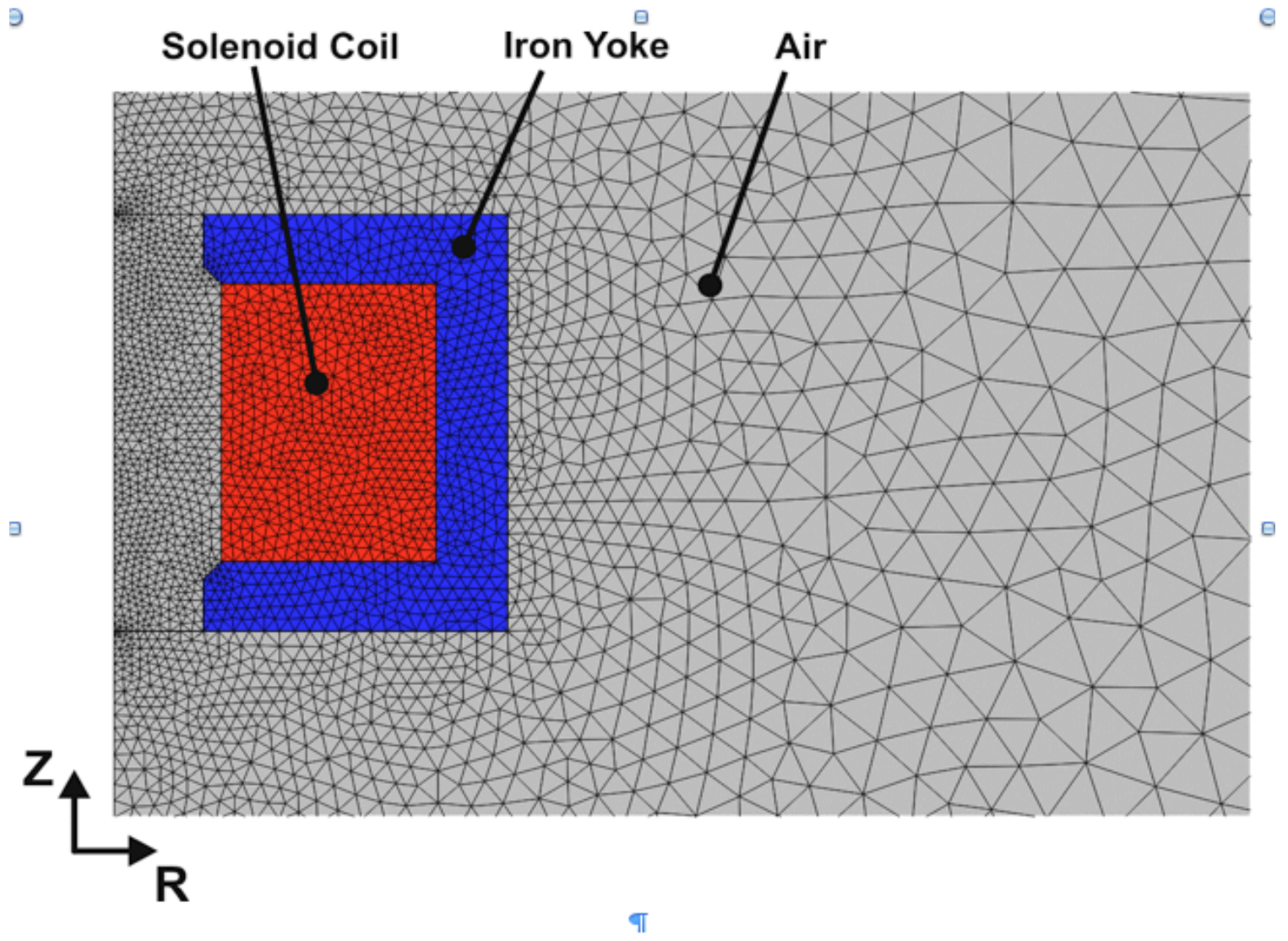

Figure 1: Example FEA grid of a solenoid magnet. था

Consider first a Poisson equation describing an electric potential function $\Phi_{e}$ :

$$
\vec{\nabla} \cdot \vec{E}=-\vec{\nabla} \cdot \vec{\nabla} \Phi_{e}=\frac{\rho}{\varepsilon_{0}}
$$

Such an equation,

$$
-\left(\frac{\partial^{2}}{\partial x^{2}} \Phi_{e}+\frac{\partial^{2}}{\partial y^{2}} \Phi_{e}+\frac{\partial^{2}}{\partial z^{2}} \Phi_{e}\right)=\frac{\rho}{\varepsilon_{0}},
$$

can be easily discretized for computations. In order to define $\Phi_{e}$, boundary conditions are required; these may be either assigned values of $\Phi_{e}$ or its normal derivative on a surface. In all electrostatic field examples it is essential that the potential is defined at least at one point in the domain, otherwise an infinite number of solutions could be generated by adding an arbitrary constant to a particular solution. 
The situation is different for magnetic problems. In the magnetostatic limit, Maxwell's equations reduce to

$$
\vec{\nabla} x\left(\frac{\vec{B}}{\mu}\right)=\vec{j} \quad, \vec{\nabla} \cdot \vec{B}=0 .
$$

Since $\vec{B}$ is not "curl free", it follows that it cannot be represented everywhere by the gradient of a scalar potential as in the electrostatic case. It is convenient to split the total field into two parts, a conductor source field and a gradient of a scalar potential, in order to obtain a description of the field in terms of a simple scalar potential:

$$
\vec{H}=\vec{H}_{s}-\vec{\nabla} \Phi \text { with } \vec{\nabla} \cdot \vec{H}_{s}=0 \text { (reduced scalar potential), }
$$

The conductor source field can be evaluated separately using the general form of the Biot-Savart law - see section 21:

$$
\vec{H}_{s}=\frac{1}{4 \pi} \int d^{3} r^{\prime} \frac{\vec{j}^{\prime} x\left(\vec{r}-\vec{r}^{\prime}\right)}{\left|\vec{r}-\vec{r}^{\prime}\right|^{3}}
$$

This is the decomposition of $\vec{H}$ into components produced by the wire and the magnetization density presented in section 16 - see eqns. (16.9) and 16.19). The field $\vec{H}_{s}$ is identical with $\vec{H}^{J}$. However, here we wish to compute $\Phi$ with $\vec{H}_{s}$ considered to be known. Introducing the scalar permeability $\mu$ and combining $\vec{\nabla} \cdot \vec{B}=0$ with the above two equations, we have the partial differential equation for $\Phi$ :

$$
\vec{\nabla} \cdot \mu \vec{\nabla} \Phi-\vec{\nabla} \cdot \mu \vec{H}_{s}=0
$$

with $\vec{H}_{s}$ calculated from eqn. (19.6).

The splitting of the magnetic field into a "curly" source part and a reduced scalar potential part has finally reduced the problem to a purely scalar one. Equation (19.7), like the Poisson equation for electrostatic fields, can be solved using the finite element method. Unfortunately, this method leads to large computational errors, especially in volumes where $\vec{H}_{s}$ and $\vec{\nabla} \Phi$ strongly cancel each other. This difficulty can be avoided when currents are not flowing in the magnetic materials. Exterior to the volumes where current flows the total field can be represented using the "total scalar potential" $\Psi$ :

$$
\vec{H}=-\vec{\nabla} \Psi \text { (total scalar potential), }
$$

which is just the scalar potential $\phi$ introduced in section 5, and which satisfies:

$$
\vec{\nabla} \cdot \mu \vec{\nabla} \Psi=0
$$


By combining the two representations (the total and the reduced scalar potentials) cancellation difficulties can be completely avoided. Therefore, the minimal combination for magnetic field simulations consists in using the reduced potential plus $\vec{H}_{s}$ only inside volumes where currents flow and using the total scalar potential everywhere else. On the interface between the total and reduced potential spaces the two potentials are linked together (internally in the computer code) by applying the conditions of normal B and tangential $\mathrm{H}$ continuity. This procedure has important consequences for setting up the simulation model. The reduced potential volume should completely enclose any coils in such a way that it is not possible to find a closed path in total potential which encloses a non-zero current. In other words any closed contour integral of $\mathrm{H}$ through any total scalar region must vanish, since otherwise the solution would be multi-valued.

\subsection{Solution strategy in $2 D$}

In 2D simulations, using either cylindrical or rectangular coordinates, the solution strategy for Maxwell's equations can be dramatically simplified compared to the general 3D strategy outline above. Since the primary topic of this report is the calculation of axisymmetric solenoid systems, we will describe the 2D method in more detail, and will give a few computation examples here and in the next chapter. Still, for a solenoid-based particle accelerator design, 3D simulations would be necessary in regions where several solenoid beamlines are transversely merged into a single beamline, or where solenoid lead effects become critical.

By using a vector potential, $\vec{B}=\vec{\nabla} x \vec{A}$, we can rewrite the relevant Maxwell's equation in the magnetostatic limit:

$$
\vec{\nabla} x\left(\frac{1}{\mu} \vec{\nabla} x \vec{A}\right)=\vec{j}
$$

Imposing gauge condition $\vec{\nabla} \cdot \vec{A}=0$, and taking into account the now two-dimensional symmetry of the physical geometry, equation (19.10) can be rewritten:

$$
\frac{\partial}{\partial z} \frac{1}{\mu} \frac{\partial A}{\partial z}+\frac{\partial}{\partial r} \frac{1}{\mu} \frac{1}{r} \frac{\partial(r A)}{\partial r}=-j,
$$

Where only the $\theta$ components $(\mathrm{A}, \mathrm{j})$ are present. In two dimensions Maxwell's equations thus reduce to single equation, which can be easily solved by standard computational methods. Contrary to three-dimensions, where the model geometry has to be divided into regions of "total" and "reduced" scalar potentials to gain a numerical scalar potential description, the equation to be solved in 2D is automatically of an effectively scalar nature. No division into different regions has to be performed in 2D. The magnetic field components are simply

$$
B_{r}=-\frac{\partial A}{\partial z}, B_{z}=\frac{1}{r} \frac{\partial(r A)}{\partial r} .
$$


This has an important consequence: Contrary to three dimensions, in a planar twodimensional simulation the equipotential lines of the vector potential are equal to the magnetic field lines (for axisymmetric simulations a modified potential $r \cdot A$ has to be used). That fact makes two-dimensional simulations much more intuitive and accessible from a practical point of view compared to any three-dimensional simulation.

\subsection{Example}

Figure (2) shows a typical axisymmetric model setup of a solenoid with an iron enclosure. Figure (3) shows the finite element mesh as generated by Tosca ${ }^{\circ}-2 \mathrm{D}$. The material properties must be defined in the code. For air (or vacuum) and the copper conductor the relative permeability is set to 1 , for the return yoke a typical B-H magnetization curve for 1010 carbon steel, as shown in figure 4, is used.

As shown in figure 5, correct boundary conditions have to be set to solve the problem numerically. For axisymmetric simulations the center axis requires a "tangential field only" (Dirichlet) boundary condition. The air volume enclosing the region of interest has to be chosen as large as possible to minimize the effect of the far-end boundary conditions. It is always a good practice to check that the far-field boundaries have been placed far enough by changing the normal field to tangential field boundaries. The change in the magnetic field in the region of interest should be minimal, otherwise the outer boundaries have not been moved far enough out. In the example the current density in the coil field region has been set to $4.7 \times 10^{6} \mathrm{~A} / \mathrm{m}^{2}$. That information is stored in a binary database file which is processed by the analysis module of the Tosca ${ }^{\circ}$ code. 


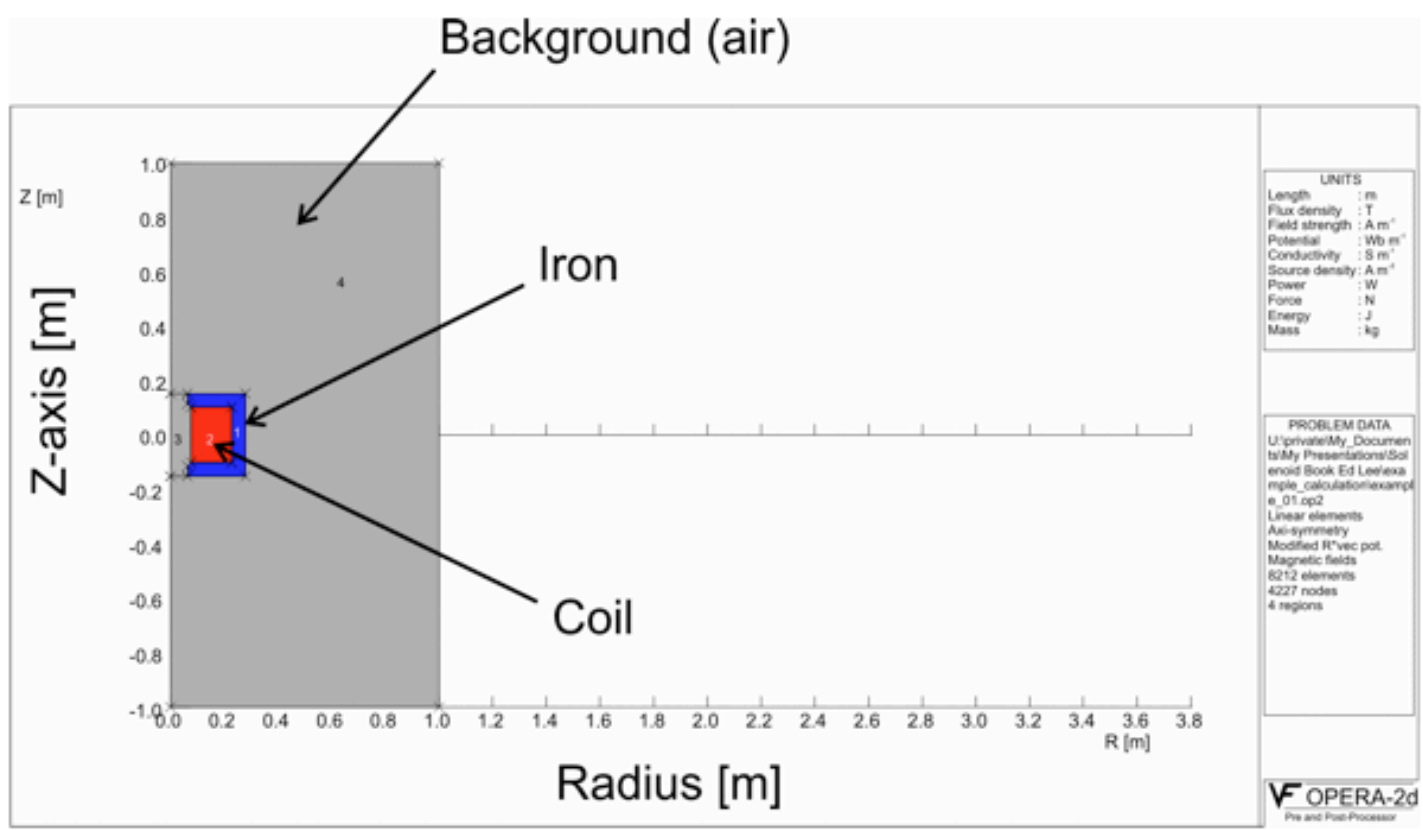

Figure 2 : Axisymmetric Tosca $^{\circ}-2 \mathrm{D}$ simulation setup. As a first step, polygons must be drawn resembling the model geometry of interest. The background air area must be chosen large enough that the model boundaries don't influence the solution in the area of interest. Tा

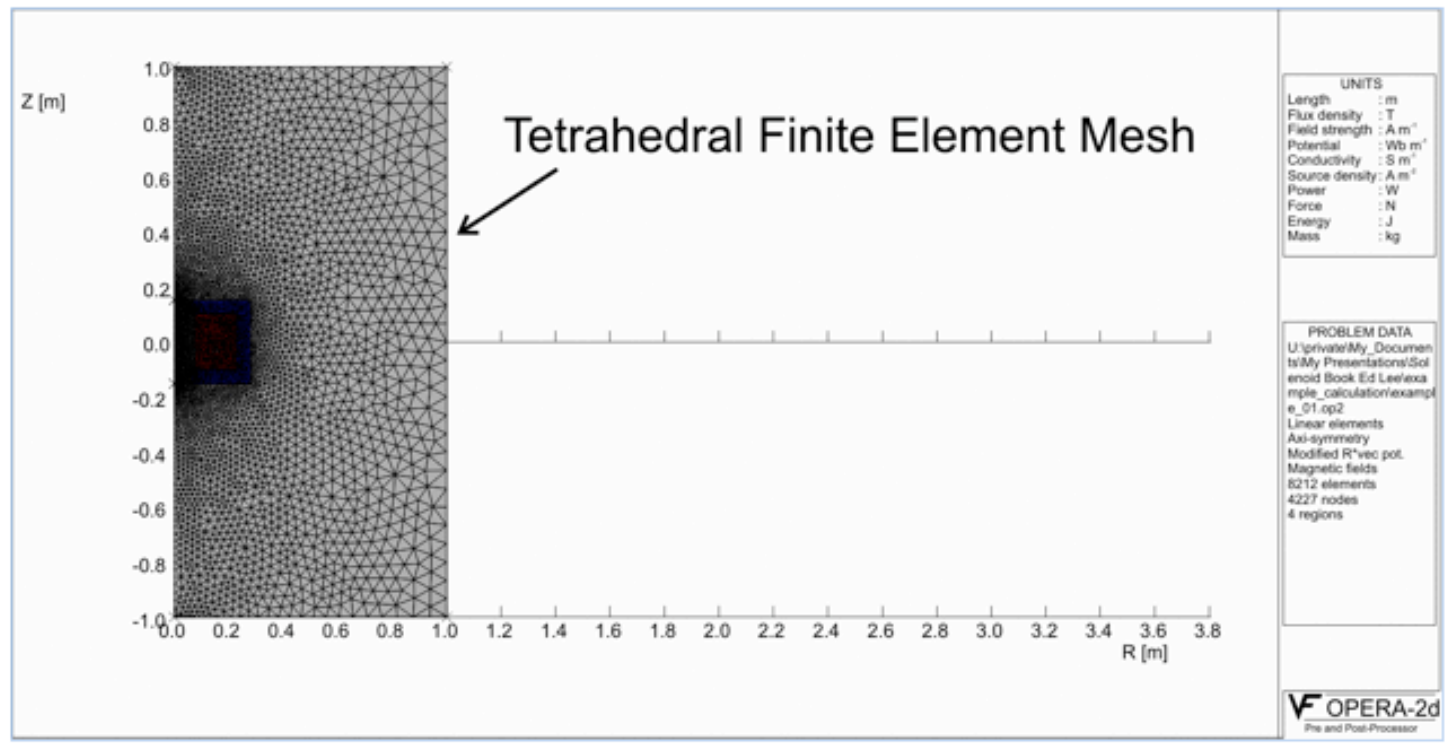

Figure 3: Tetrahedral finite element mesh as generated by the Tosca-2D mesh generator. The mesh has been chosen to be finer on axis and inside the solenoid area of interest. It gradually becomes coarser towards the outside model boundaries in order to minimize computation time. था 


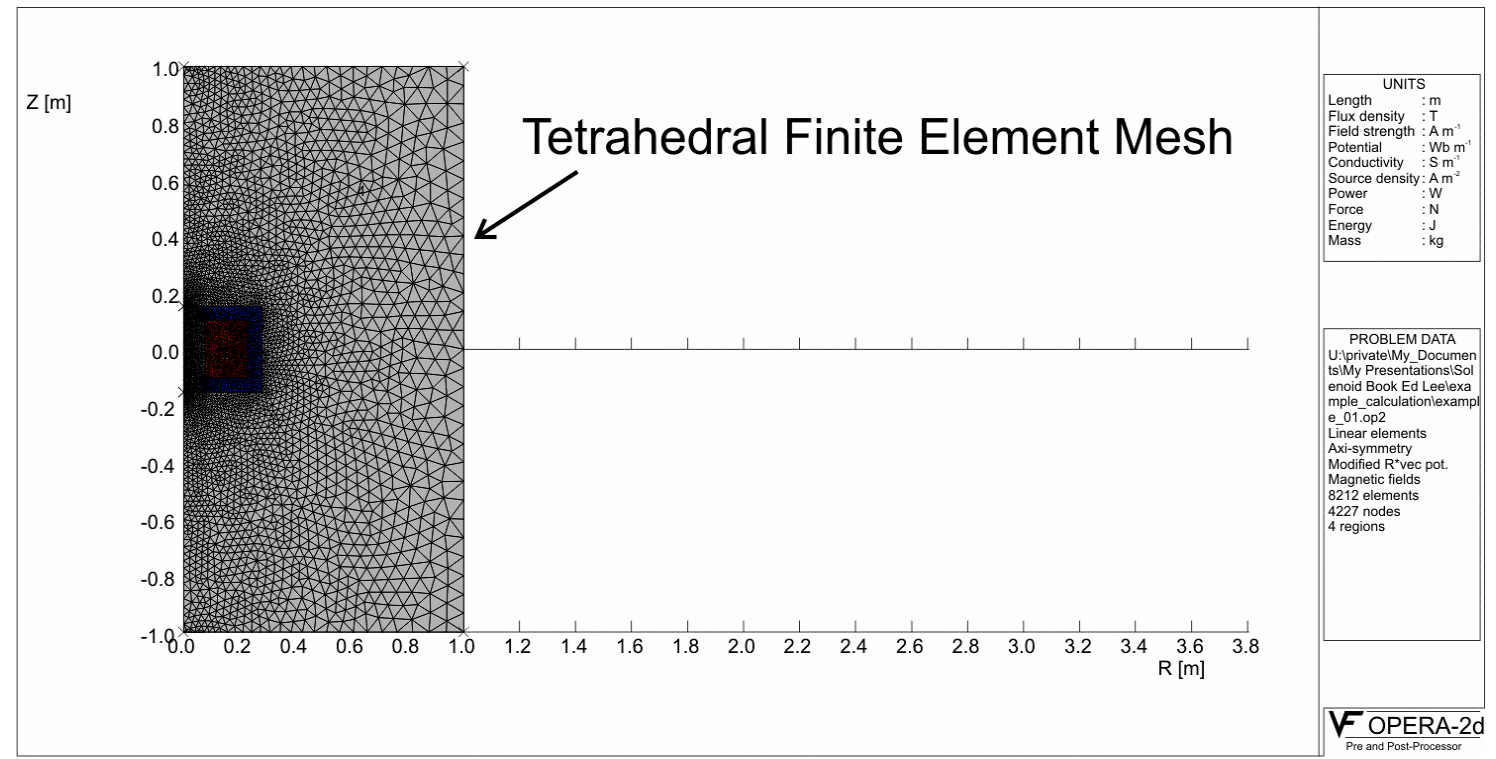

Figure 3: Tetrahedral finite element mesh as generated by the Tosca-2D mesh generator. The mesh has been chosen to be finer on axis and inside the solenoid area of interest. It gradually becomes coarser towards the outside model boundaries in order to minimize computation time.

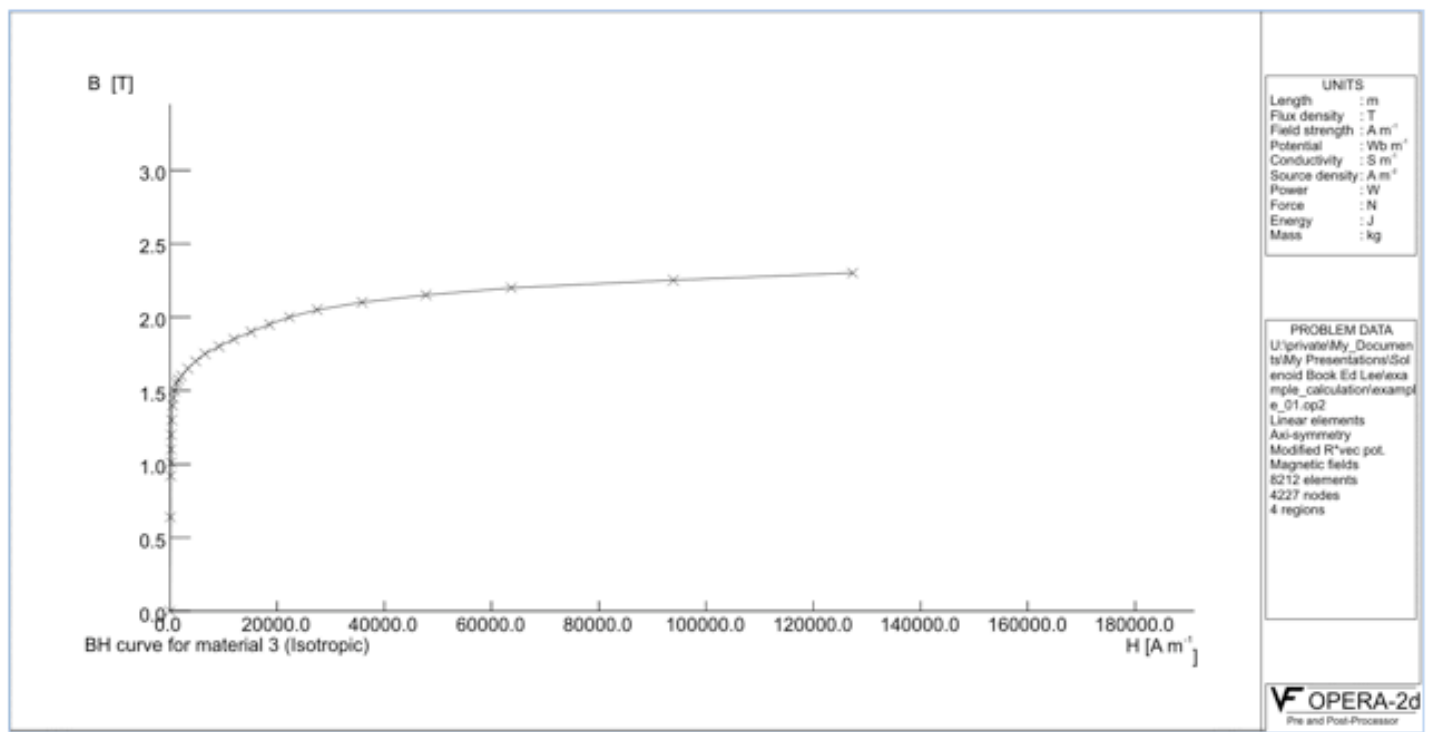

Figure 4: Each material inside the Tosca model has to be defined. For instance, the relative permeabilities of air and the coil have to be set to 1 . For the coil region, a current density must be prescribed. Non-linear material properties are defined through permeability tables as shown in this figure. I 


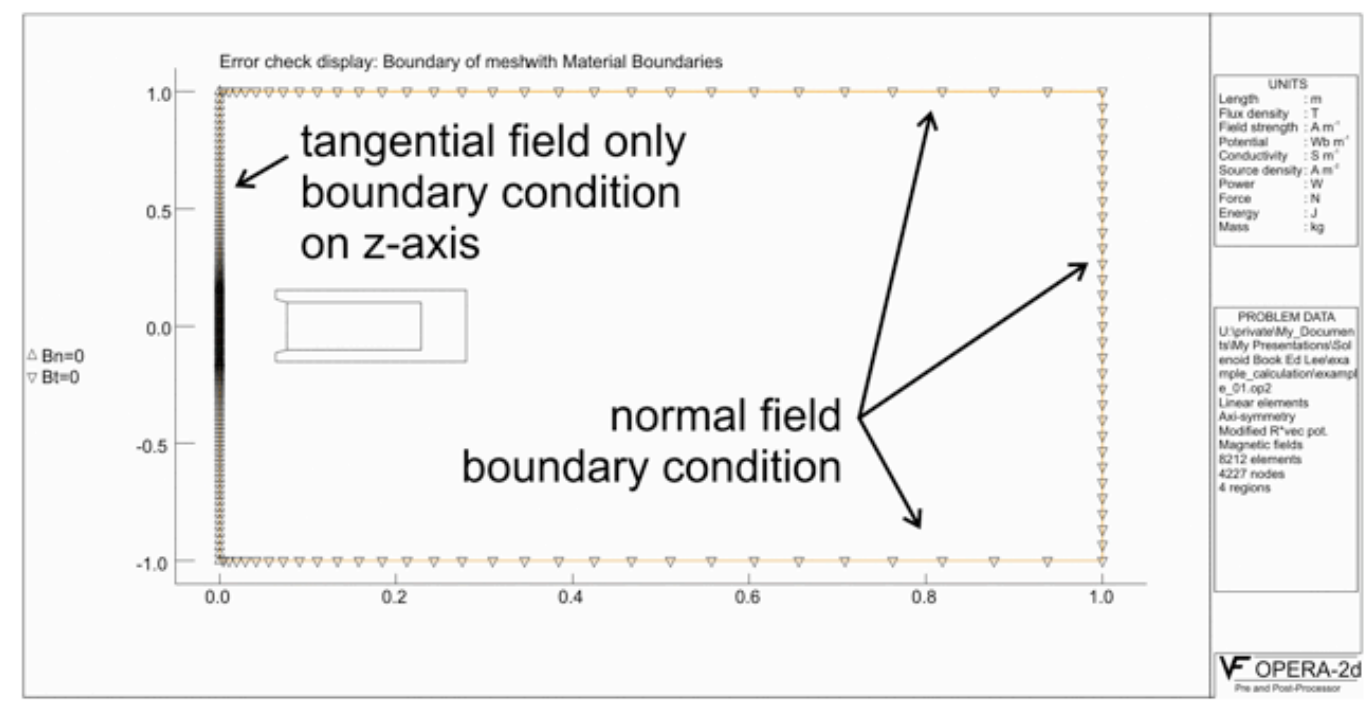

TI Figure 5 : Boundary conditions have to be set to solve the problem numerically.

था For axisymmetric simulations the center axis requires a "tangential field only"

था (Dirichlet) boundary condition. The air volume enclosing the region of interest has

I to be chosen as large as possible to minimize the effect of the far-end boundary

था conditions. था

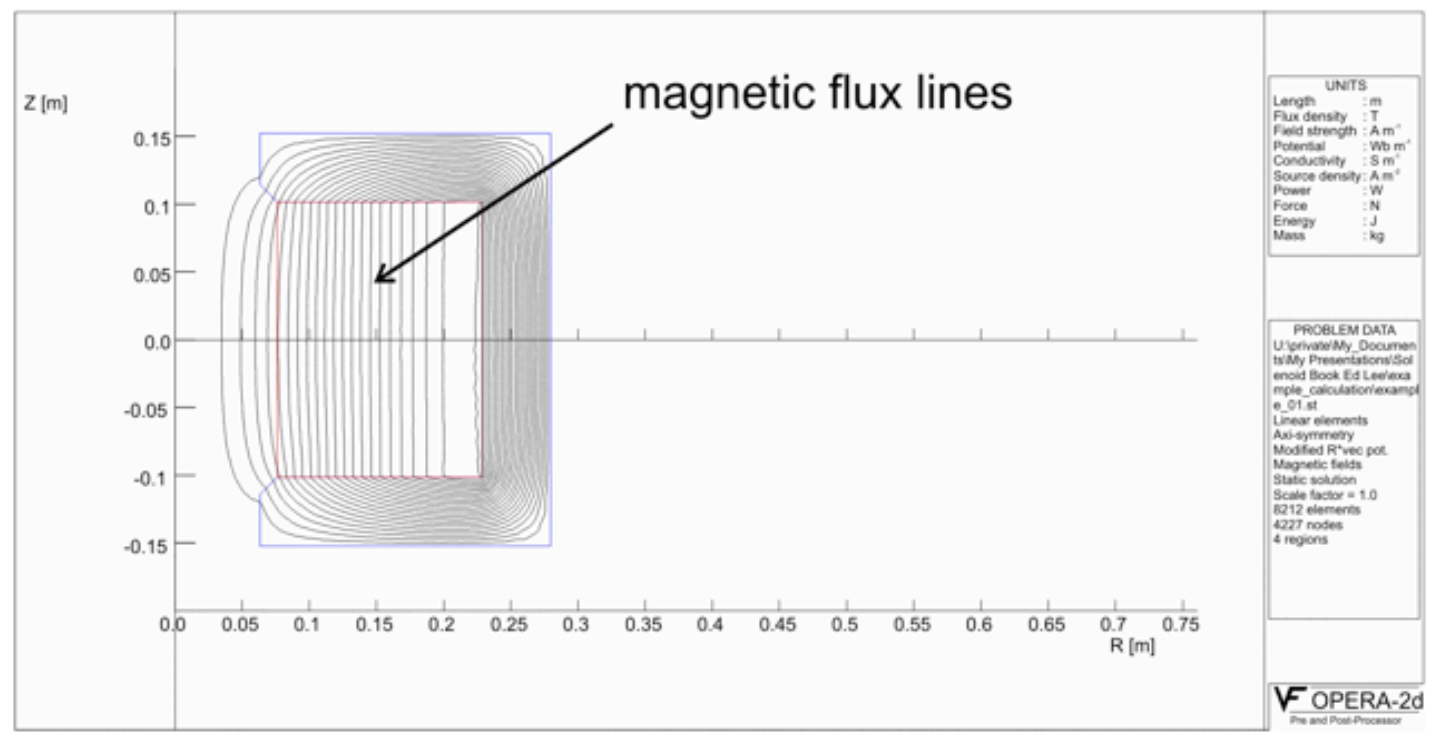

Figure 6: Model geometry with the magnetic flux lines displayed. था

Computation of the vector potential proceeds iteratively for each mesh point. The computation proceeds in the iron region with the determination of vector potential, magnetic induction, and permeability at each mesh point. The vector potentials A are first computed at all points by assuming a constant value of permeability, then the components of the induction $\mathrm{B}$ are determined as partial derivatives of $\mathrm{A}$, and the permeability values as a function of the absolute values of B are read from a B-H curve table. Then the whole process is repeated with adjusted permeability values: cycling is continued until the changes in permeability are all below a specified value. 
Once the field computation has been performed the database is read into a postprocessor for further evaluation. Figure 6 shows the model geometry with the magnetic flux lines displayed. Figure 7 shows contour zones of the total magnitude of the magnetic flux density, and figure 8 shows a read out of the magnetic field on axis.

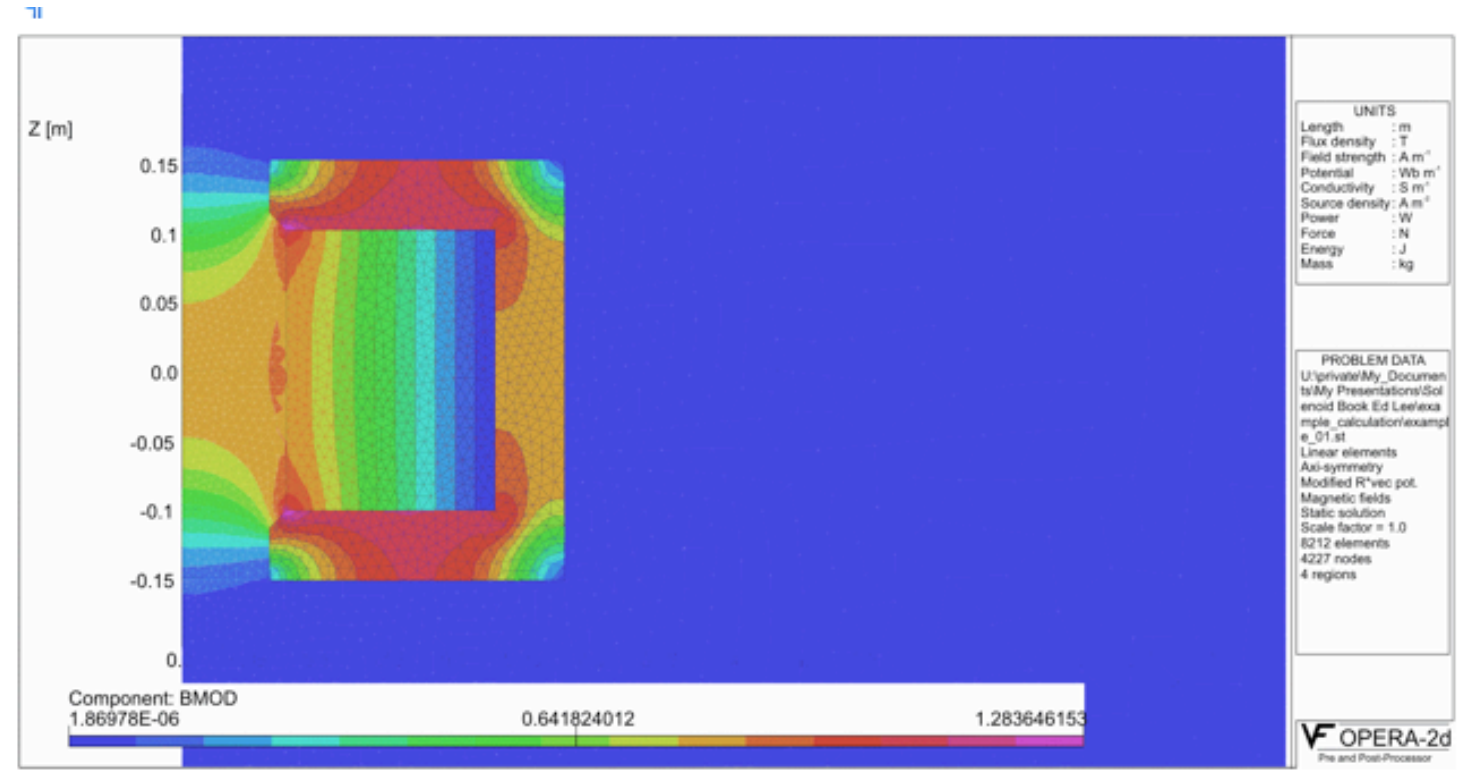

Figure 7: Colored contour zones of the absolute magnitude of the magnetic flux density. As can be clearly seen, most of the magnetic return flux is concentrated in the iron yoke, which starts to saturate. Finite element analysis is best suited for predicting the magnetic field distribution in cases where saturated materials with non-linear magnetic properties are present. ๆ 


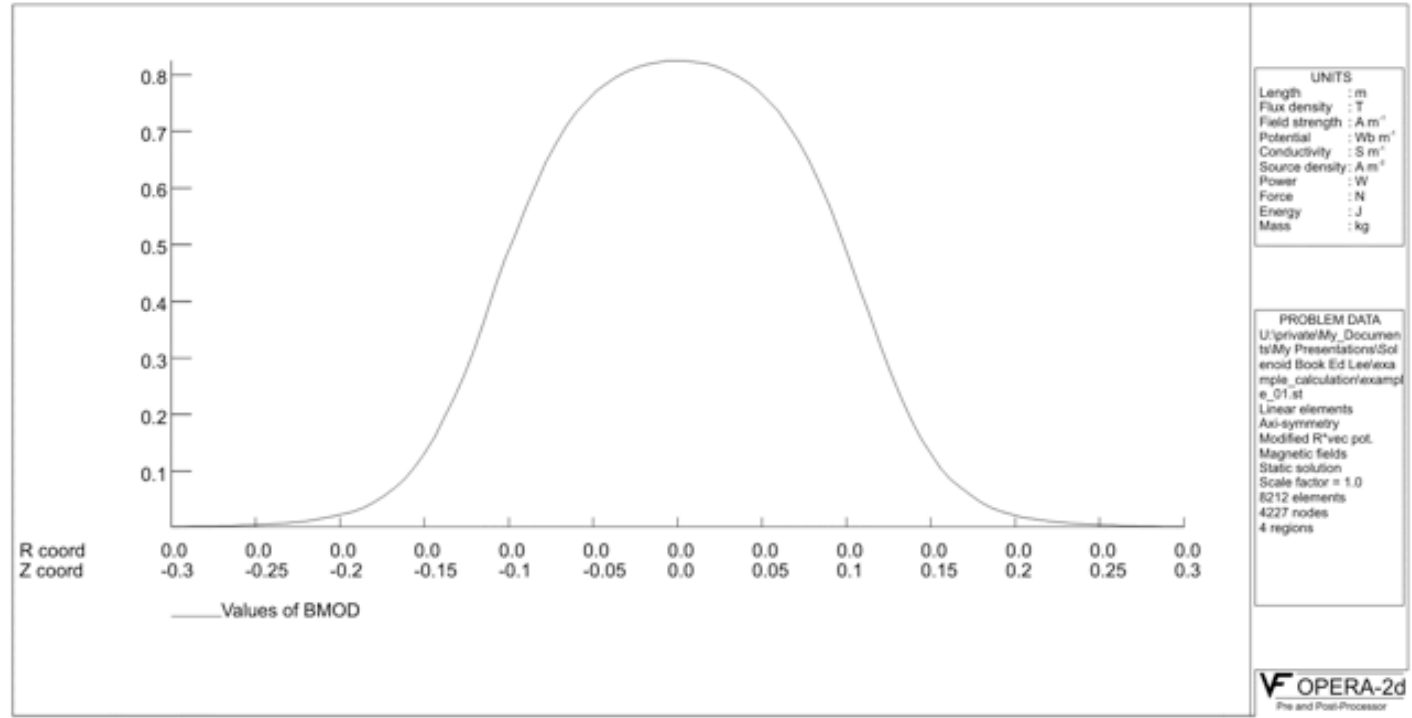

Figure 8: Magnetic flux density in Tesla along the solenoid axis. था 


\section{Application of Tosca ${ }^{\odot}$ to Induction Linac Design}

\subsection{Introduction}

Recently there has been renewed interest in relatively low energy modular induction accelerators to drive close-coupled HIF targets ( $\sim 75 \mathrm{MV}$ acceleration voltage of $\mathrm{Ar}^{8+}$, corresponding to $600 \mathrm{MeV}$ kinetic energy). Similar, smaller-scale designs are suggested for a possible facility to perform high energy density physics experiments. These designs utilize solenoid beam transport because of its favorable scaling at the desired ion mass and kinetic energy.

An example "modular solenoid driver" 20-1 would consist of 40 beamlines (20 on each side of the final focus chamber). Each beamline would have a high current $\mathrm{Ar}^{8+}$ injector and a pre-bunch section to compress the beam for injection into a solenoid-based modular induction linac. The total linac length would be only 75 meters. The accelerated beams, which have an approximately $15 \%$ energy tilt imposed, compress in a neutralized drift section ( $\sim 260$ meters long) before final radial compression inside a liquid-vortex flibe chamber with a solenoid focusing magnet set.

Figure 1 shows the mechanical layout of the high-energy end of such a modular solenoid linac. The main dimensions of a linac cell are given in figure 2 . The transport solenoids are superconducting $\mathrm{Nb}_{3} \mathrm{Sn}$ magnets producing 12 Tesla peak axial magnetic field. Finite-element based electromagnetic simulation tools are utilized in several design aspects of the linear accelerator:

1. The solenoids are embedded inside induction cores of high permeability. To shield the solenoid return flux from the induction cores, the superconducting winding structure is enclosed by a thick, soft-magnetic steel shell. Finite element codes are reliable tools for calculating the effects of the permeable materials and allow optimization of the acceleration cell geometry.

2. The ion beams exiting the 20 modular solenoid linacs must be matched into the focusing system leading to the fusion target. Three-dimensional finite element codes can reliably calculate the dipole and higher-order field contributions of the solenoid magnetic fields at the end of the linac structure, especially if permeable materials are present to channel the linac fields' return flux.

3. Finite element codes are used to design the superconducting winding pack. They allow the exact coil dimensioning using critical current load lines.

4. If one solenoid along a linac string quenches, the forces on the neighboring solenoids will be imbalanced. Finite element codes allow one to calculate these forces and to dimension a sufficient support system. The electromagnetic simulation models are directly linked with structural models to design the solenoid clamping structure. 


\section{MODULAR INDUCTION LINAC}

HIGH ENERGY END

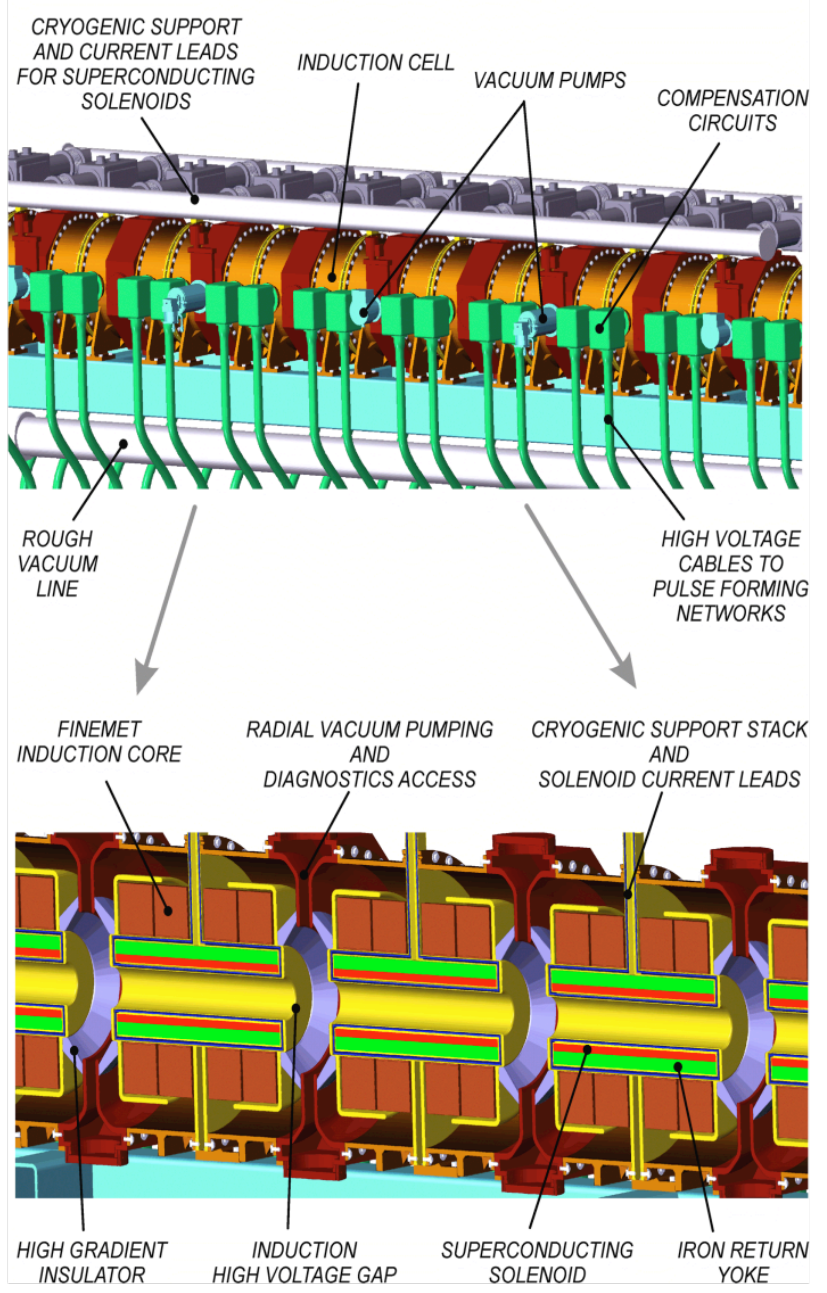

Figure 1: A modular solenoid driver would use strong solenoids for focusing the heavy ion beam. 


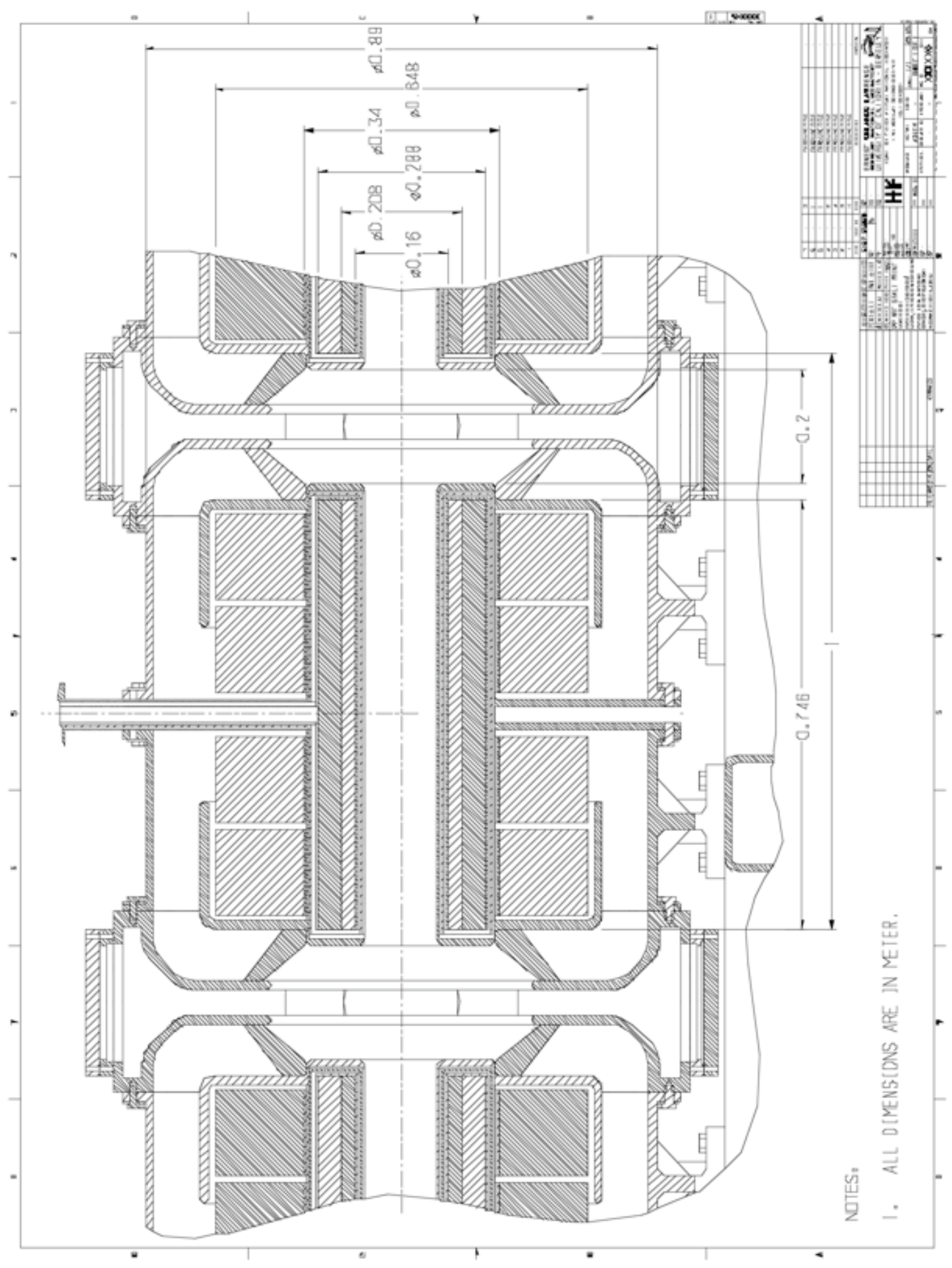

Figure 2: 2D drawing showing the main dimension of the driver induction cell. 


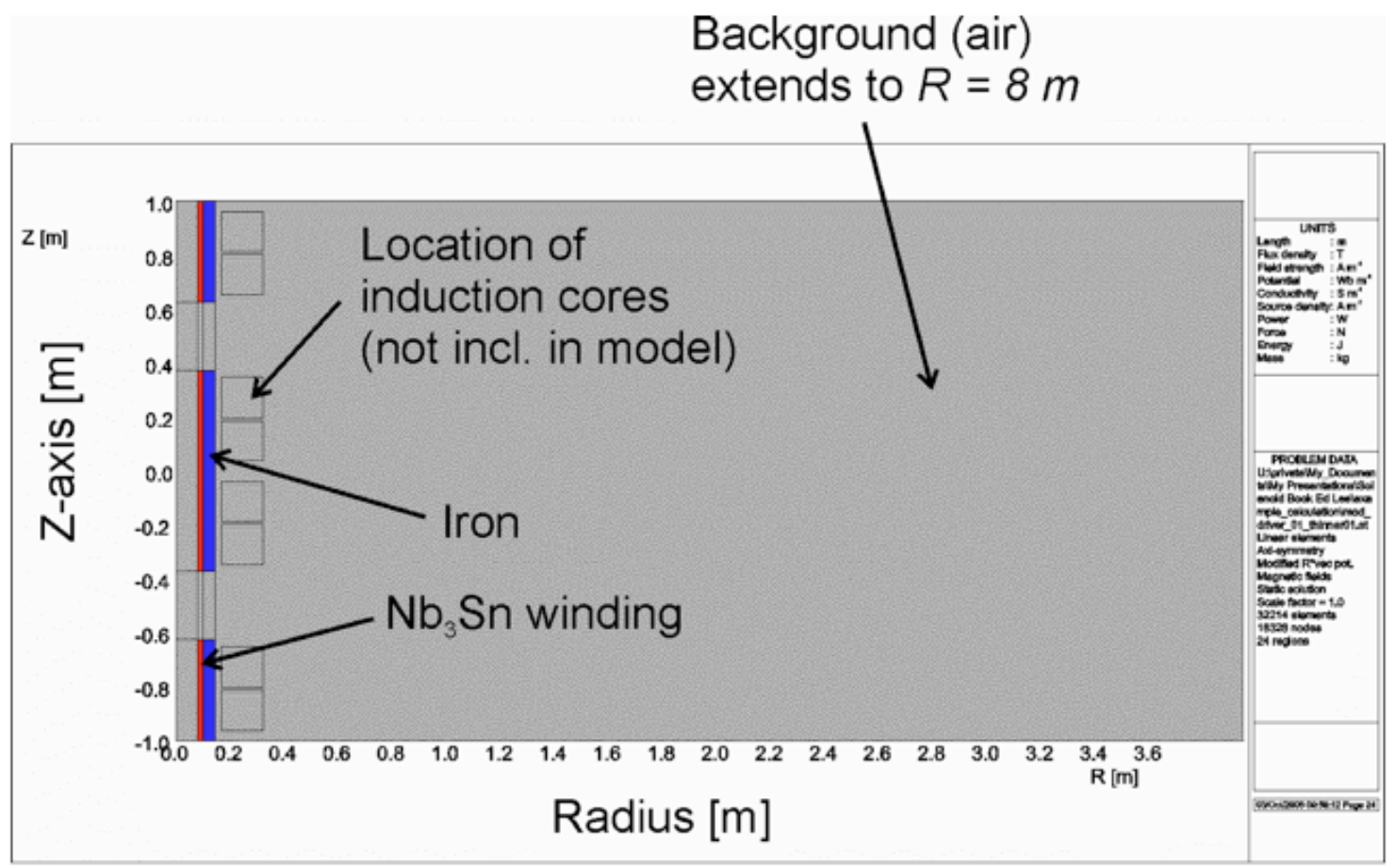

Figure 3: Example FEA setup of a modular solenoid driver.

\subsection{Example}

As an example of how electromagnetic modeling can be utilized to optimize the modular solenoid driver architecture, we look at the effect of adding iron outside the superconducting solenoid winding. We will also discuss how to specify the superconducting winding structure.

Figure 3 shows a simulation setup for an infinitely long solenoid string consisting of a (thin) superconducting wire pack surrounded by an iron cylinder. The rest of the model consists of air with a relative permeability equal to 1 . The location of the induction cells is indicated, but we will not include their material properties in this example. The beam travels along the vertical axis, and the radial coordinate is the horizontal axis.

Such a simulation should strongly take advantage of the model symmetry. First of all the simulation is 2D axisymmetric with the beam axis as symmetry axis. Second, because of the infinite nature of the problem the top and bottom model boundaries are symmetry planes through the centers of the solenoids. The correct numerical boundary conditions are shown in figure 4 . The background air (vacuum) volume extends radially far outside to minimize the influence of the far field boundary condition, which was set to tangential $B_{r}=0$. 


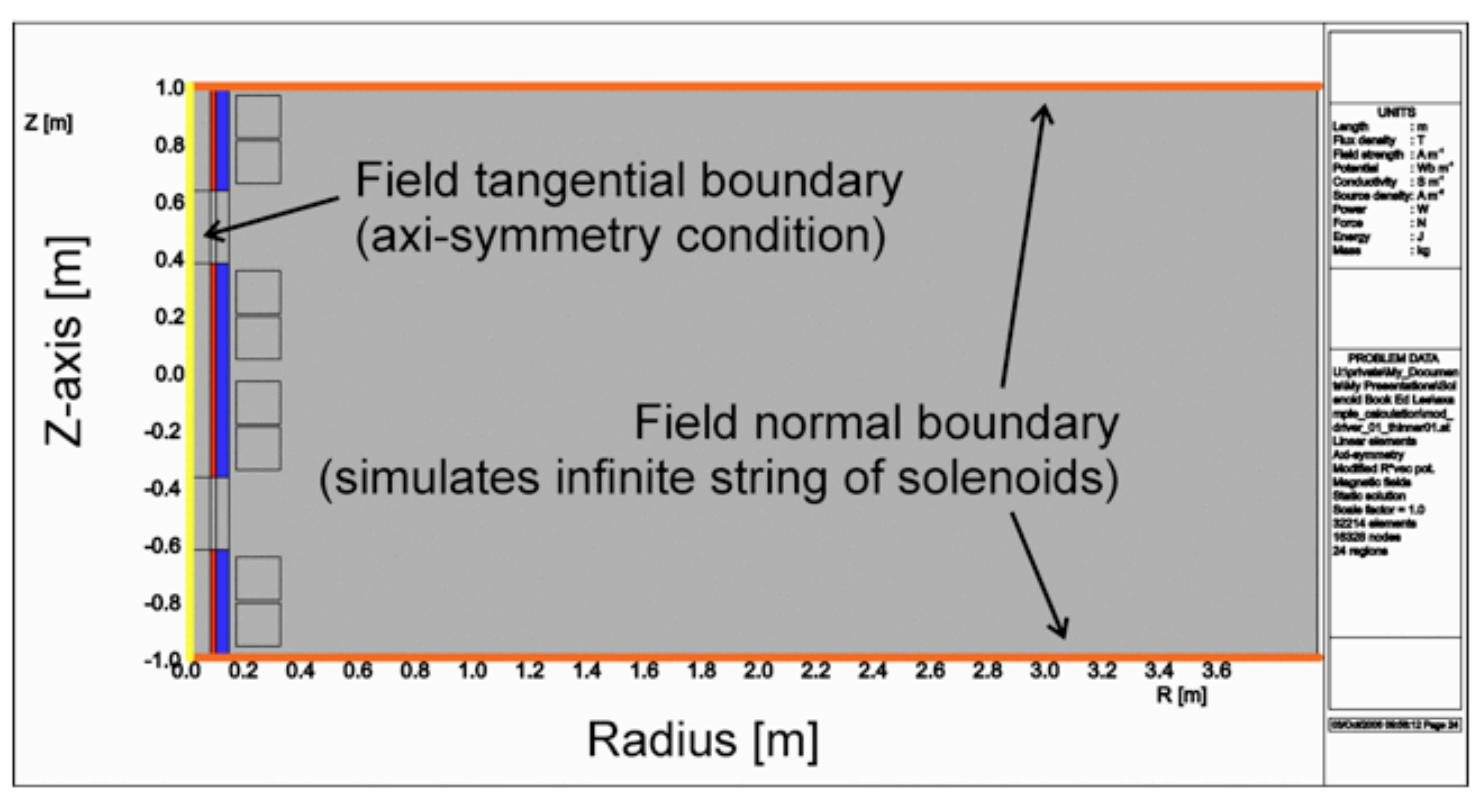

Figure 4: The FEA boundary conditions are set to simulate an infinite string of solenoids. A 2D simulation is sufficient because of the axisymmetric nature of the problem.

Figure 5 shows in more detail the region around one single solenoid and the finite element grid generated by the Tosca ${ }^{\circledR} 2 \mathrm{D}$ simulation code. To enhance the accuracy of the simulation model, regions close to the beam axis - including the solenoid winding and the iron regions - are modeled using mapped hexagonal finite elements. The background air model uses tetragonal elements, which allow gradually increasing element sizes. In that way the model can have a very fine grid around the areas of interest and a coarser grid at the far field boundaries.

After meshing the problem geometry the material and conductor properties have to be defined. Figure 6 shows the permeability curve for 1010 steel, a soft magnetic steel used for building electromagnets.

Figure 7 shows a graph of critical current densities for different superconducting cable materials [2]. The blue curve with blue squares shows the critical current line for $\mathrm{Nb}_{3} \mathrm{Sn}$ at a temperature of 4.2 Kelvin, the temperature of the liquid helium coolant. The critical current density is the maximum current density a superconducting wire can transport without loosing its superconductivity, which is dependent on the applied magnetic field. The higher the magnetic field the lower the critical current density. $\mathrm{Nb}_{3} \mathrm{Sn}$ has similar critical current densities as NbTi but can be operated at much higher applied magnetic fields.

It is important to realize that the critical current densities are shown for single stranded superconducting wire. The actual current densities used as input in the simulations to define the conductor regions are significantly lower. Figure 8 shows a 
cross sections of a few actual superconducting wires. It shows the superconducting wire strands embedded inside a copper matrix whose purpose is two-fold.
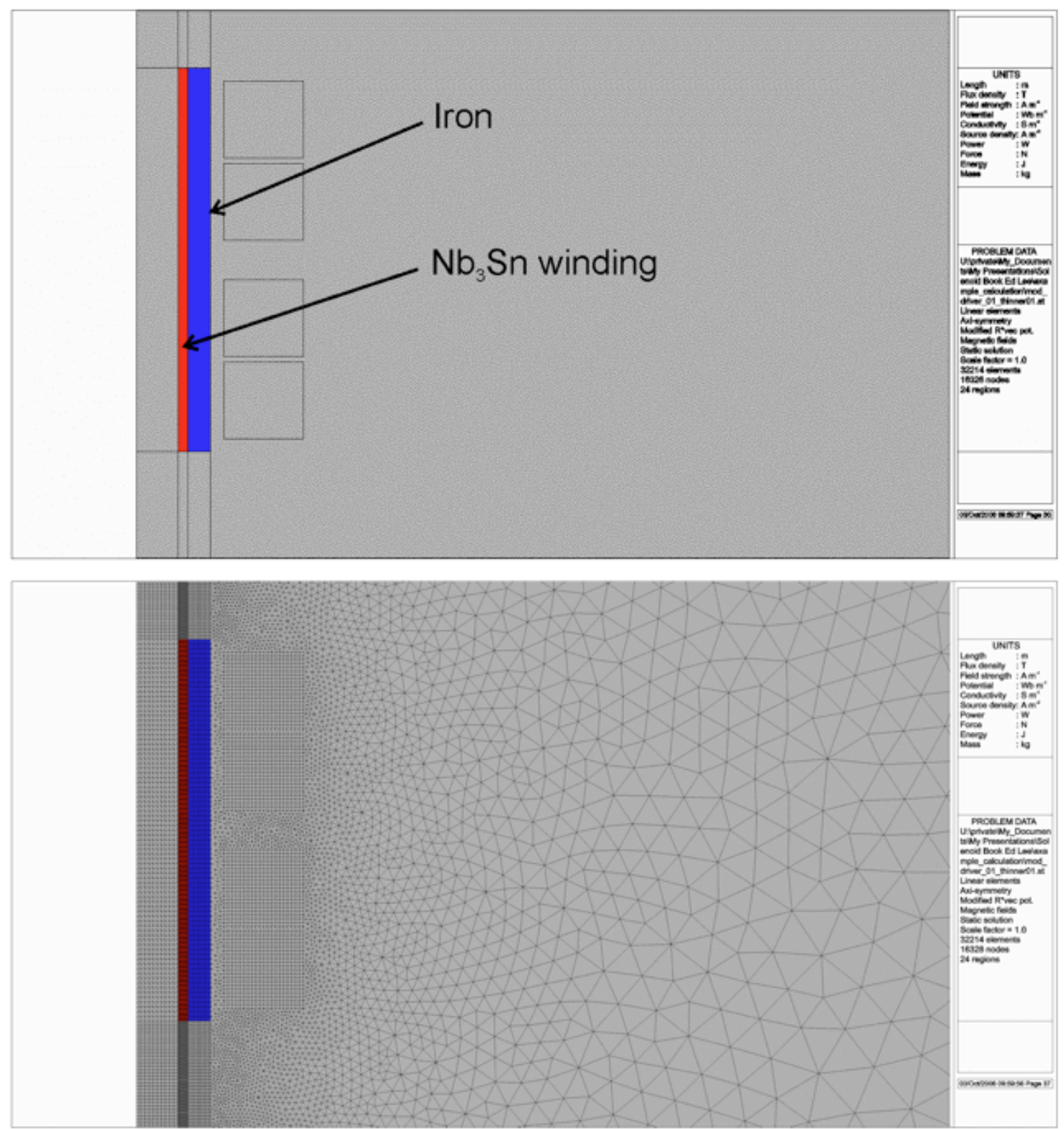

Figure 5: Example 2D FEA grid of a solenoid magnet.

First it helps to locally stabilize the superconducting wire. On a microscopic scale, the superconducting state is fluctuating, and a sufficiently sized copper matrix helps to stabilize the superconducting condition. Second, the wire has to be able to transport all the current in cases where the wire quenches and superconductivity is lost entirely. Enough copper must be available to transport the current without melting the wire. 


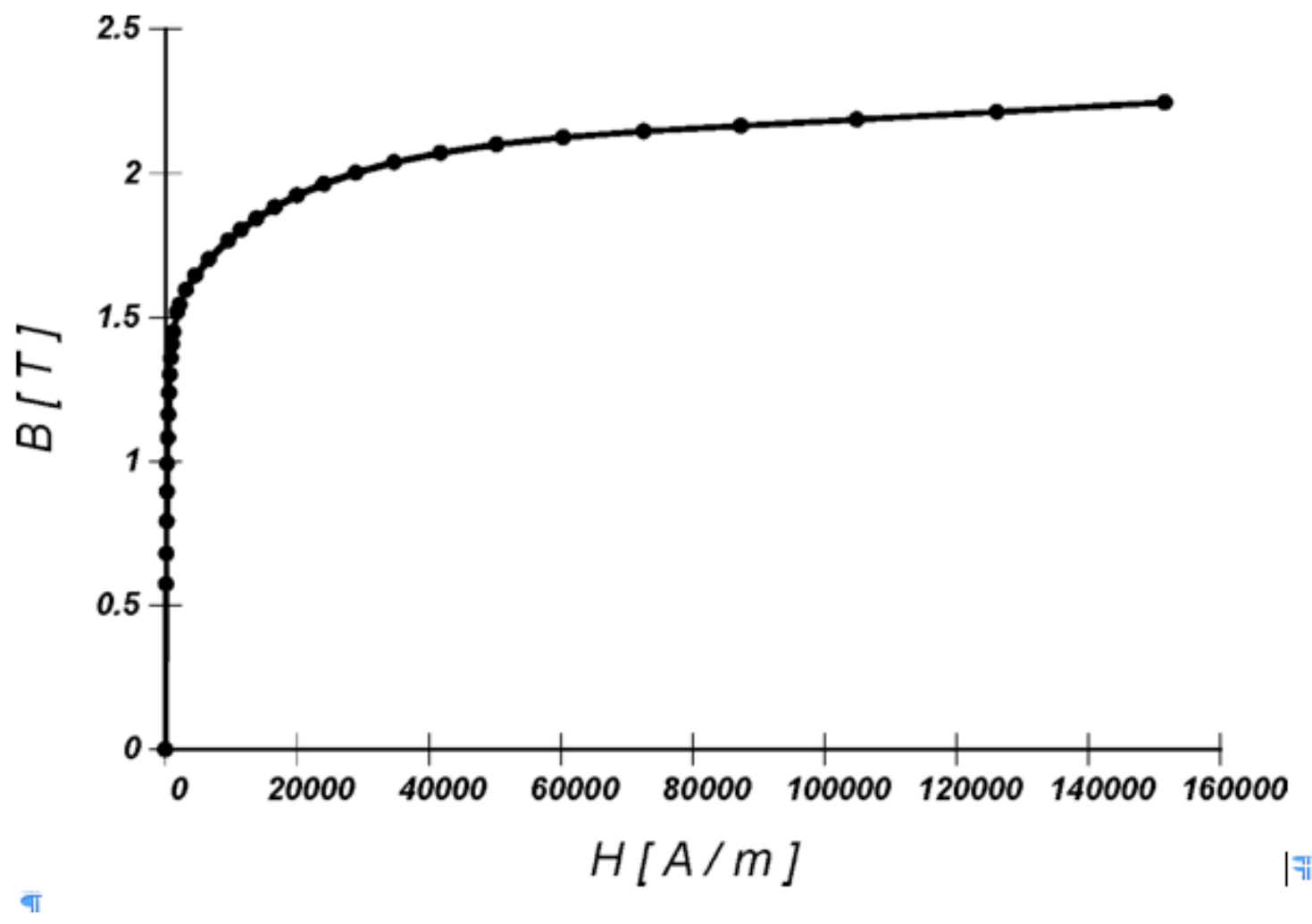

Figure 6: BH curve for 1010 steel, a soft magnetic steel used for building electromagnets.

In designing a magnet, what really matters is the overall "engineering current density". It is given by the superconducting strand critical current density times the superconducting material to copper ratio times the packing factor of the actually wound superconducting wire. Figure 8 demonstrates the packing of commercial round wires in a magnet winding.

$$
J_{\text {overall }}=\text { packing factor } \cdot J_{\text {wire }}=\text { packing factor } \cdot \frac{1}{C u / S C \text { ratio }} J_{c}
$$

with

$$
J_{\text {overall }} \ldots \quad \text { "engineering current density" }
$$

packing factor ... takes account of space occupied by insulation, eventual cooling channels, mechanical reinforcement, epoxy, etc.

$\mathrm{Cu} / \mathrm{SC}$ ratio ... ratio of copper to superconducting material Table 1 determines the maximum $\mathrm{Cu} / \mathrm{SC}$ ratio by limiting the maximum current density to be carried by the copper matrix to $1500 \mathrm{~A} / \mathrm{mm} @$ in case of a complete quench

$J_{c} \ldots \quad$ critical current density for single superconducting strand 


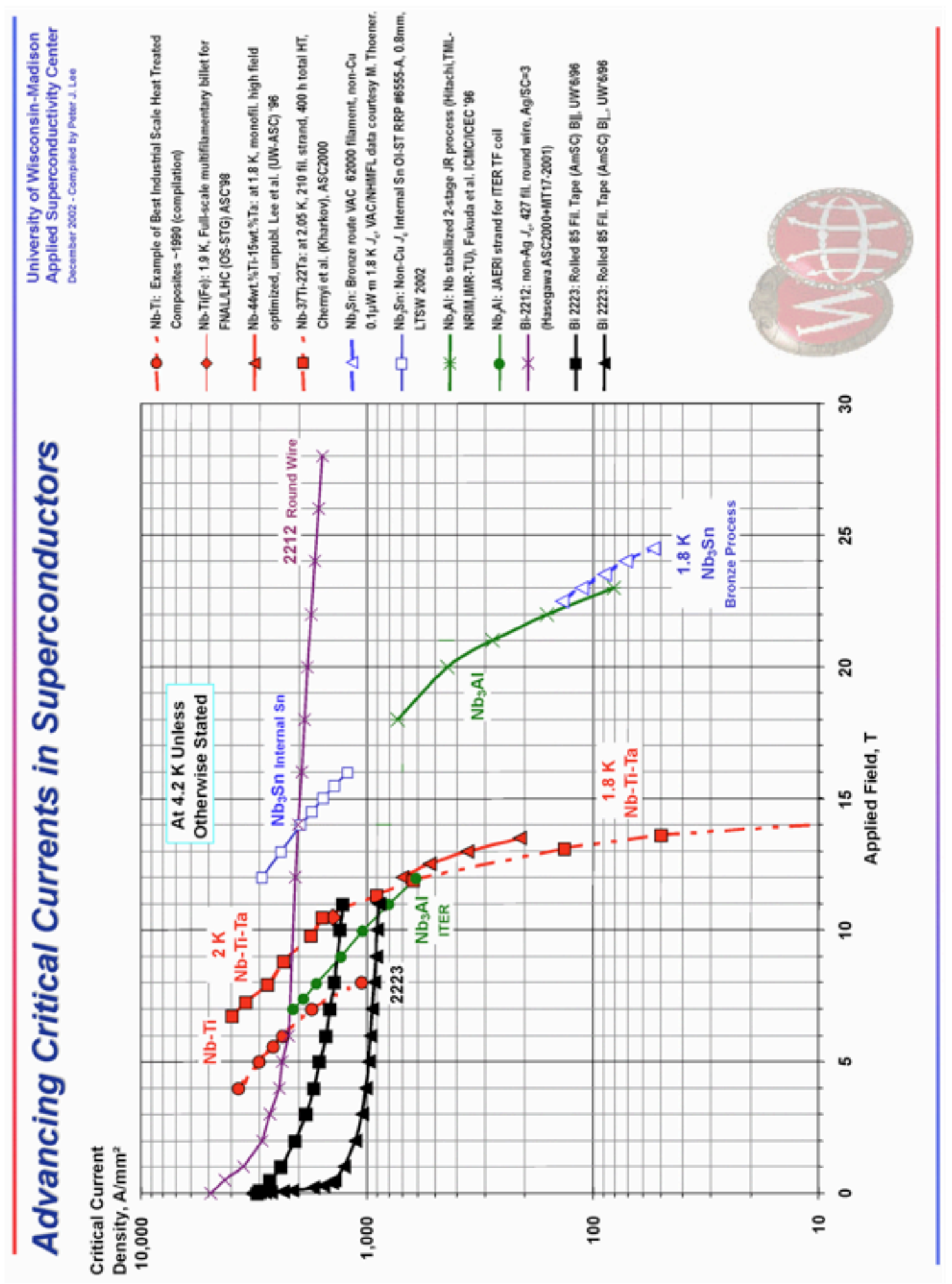

Figure 7: Critical current densities for commercial superconducting wires [2]. The blue curve with blue squares shows the critical current line for $\mathrm{Nb}_{3} \mathrm{Sn}$ at $4.2 \mathrm{~K}$. 


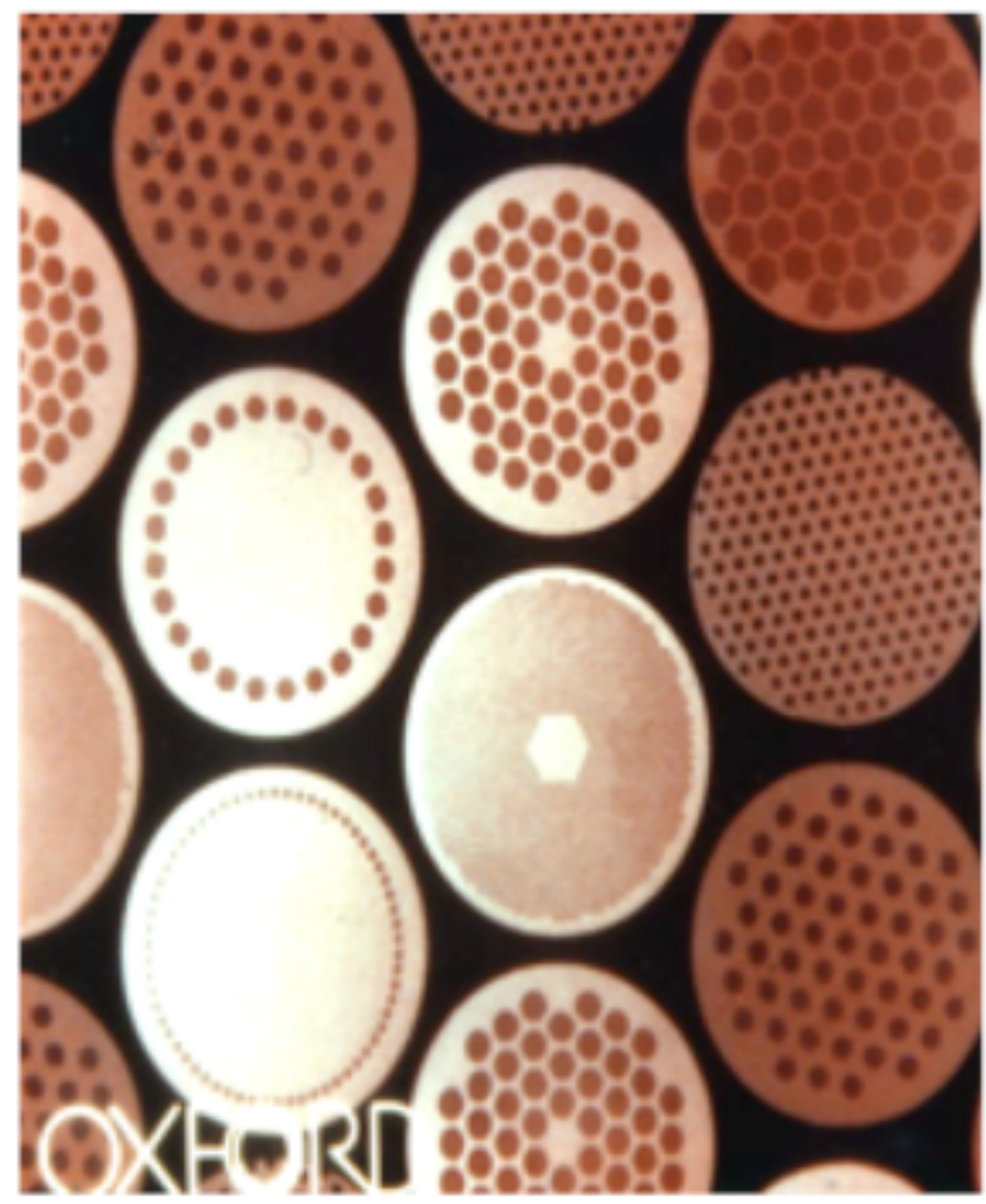

Figure 8: Cross section of various actual superconducting wires. The superconducting filaments, which are actually twisted, are embedded inside a copper matrix. Additional space around the wires is needed for insulation, structural epoxy, or cooling channels.

Table 1 summarizes the actual "engineering" current densities used to specify conductors in the simulation dependent on the applied magnetic field. An additional 30\% safety margin is applied to the engineering current densities to account for winding strain and temperature fluctuations. Depending on the actual mechanical solenoid design an even larger temperature safety margin may be necessary at higher applied magnetic fields.

Figure 9 displays the field lines of the resulting solenoid beam transport channel. Figure 10 shows the axial magnetic field strength along the beam center axis. The field ripple due to the fairly large magnet spacing, which is necessitated by the wide induction acceleration gap (1 MV/m gradient), is clearly visible. 


\begin{tabular}{|c|c|c|c|c|c|}
\hline$\stackrel{B}{B}$ & $\stackrel{\mathbf{J}_{\mathbf{c}}}{\left[\mathbf{A} / \mathbf{m m}^{2}\right]}$ & $\underset{(*)}{\mathbf{C u} / \mathbf{S C} \text { Ratio }}$ & $\begin{array}{c}\mathbf{J}_{\text {wire }} \\
{\left[\mathbf{A} / \mathbf{m m}^{2}\right]}\end{array}$ & $\begin{array}{c}\mathbf{J}_{\text {overall }} \\
{\left[\mathbf{A} / \mathbf{m m}^{2}\right]}\end{array}$ & $\begin{array}{l}\text { with } 30 \% \\
\text { margin }^{(* *)} \\
{\left[\mathrm{A} / \mathbf{m m}^{2}\right]}\end{array}$ \\
\hline 5 & 9454 & 6.30 & 1295 & 906 & 634 \\
\hline 6 & 7766 & 5.18 & 1257 & 880 & 616 \\
\hline 7 & 6431 & 4.29 & 1216 & 851 & 596 \\
\hline 8 & 5347 & 3.56 & 1171 & 820 & 574 \\
\hline 9 & 4446 & 2.96 & 1122 & 785 & 550 \\
\hline 10 & 3689 & 2.46 & 1066 & 746 & 523 \\
\hline 11 & 3048 & 2.03 & 1005 & 704 & 493 \\
\hline 12 & 2500 & 1.67 & 938 & 656 & 459 \\
\hline 13 & 2031 & 1.35 & 863 & 604 & 423 \\
\hline 14 & 1631 & 1.09 & 781 & 547 & 383 \\
\hline 15 & 1289 & 0.86 & 693 & 485 & 340 \\
\hline
\end{tabular}

${ }^{(*)} \quad$ for a maximum current density of $1500 \mathrm{~A} / \mathrm{mm}^{2}$ in the copper matrix ${ }^{(* *)} \quad$ margin for winding strain and temperature flucuations

Table 1: Engineering current densities $\left(J_{\text {overall }}\right)$ for $\mathrm{Nb}_{3} \mathrm{Sn}$ at different magnetic fields. 


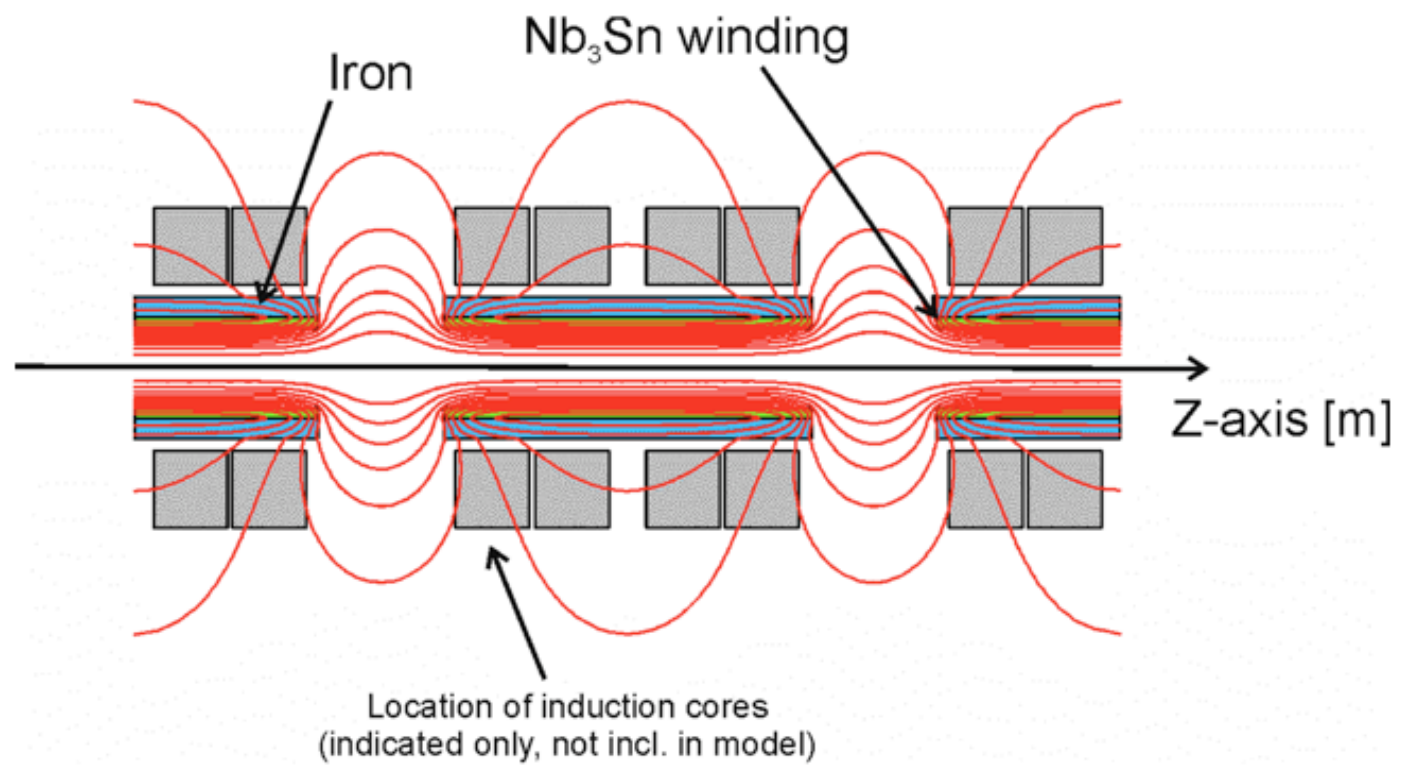

Figure 9: Magnetic flux lines for a string of solenoids.

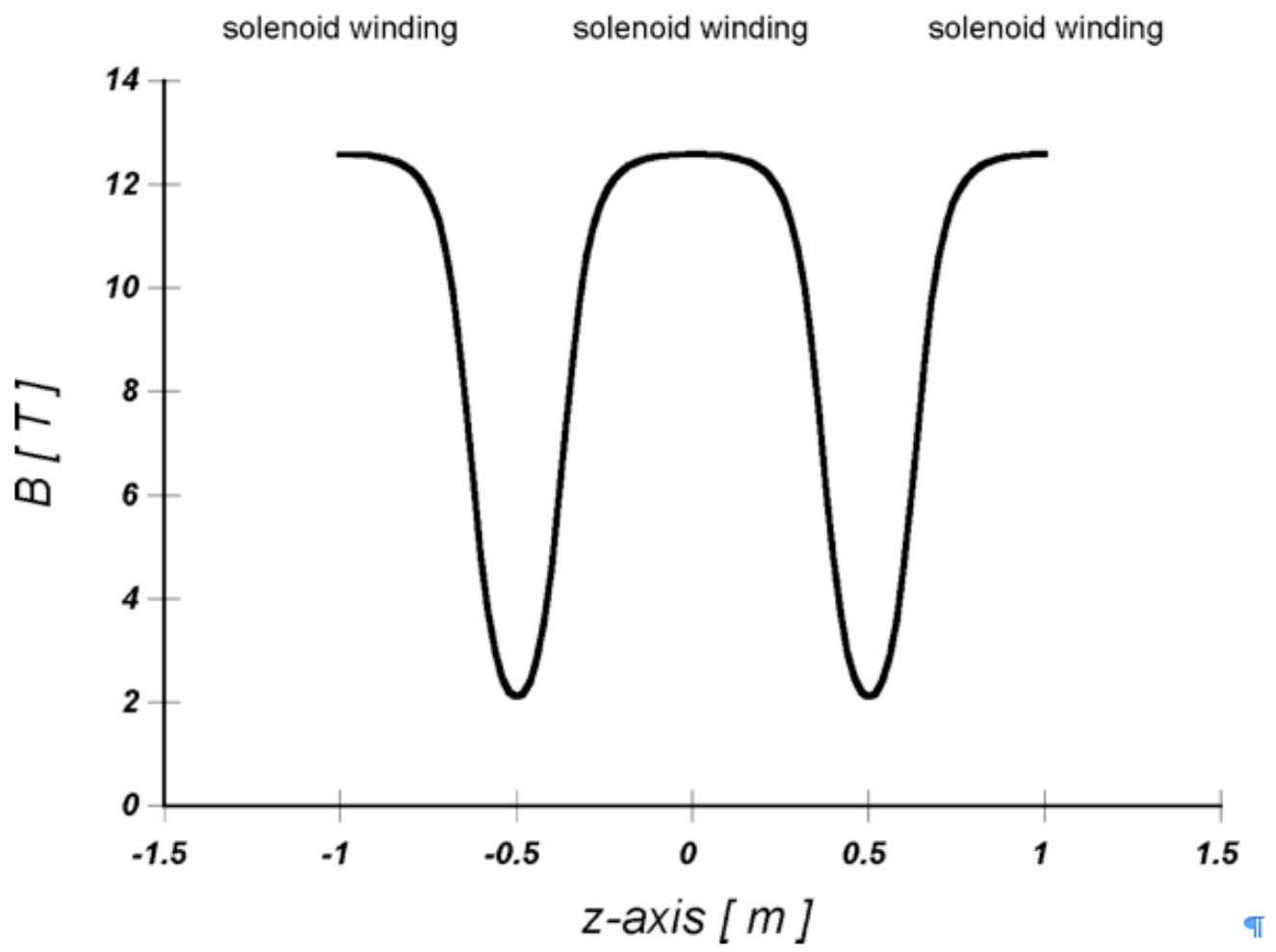

Figure 10: Magnetic field along the beam center axis. 


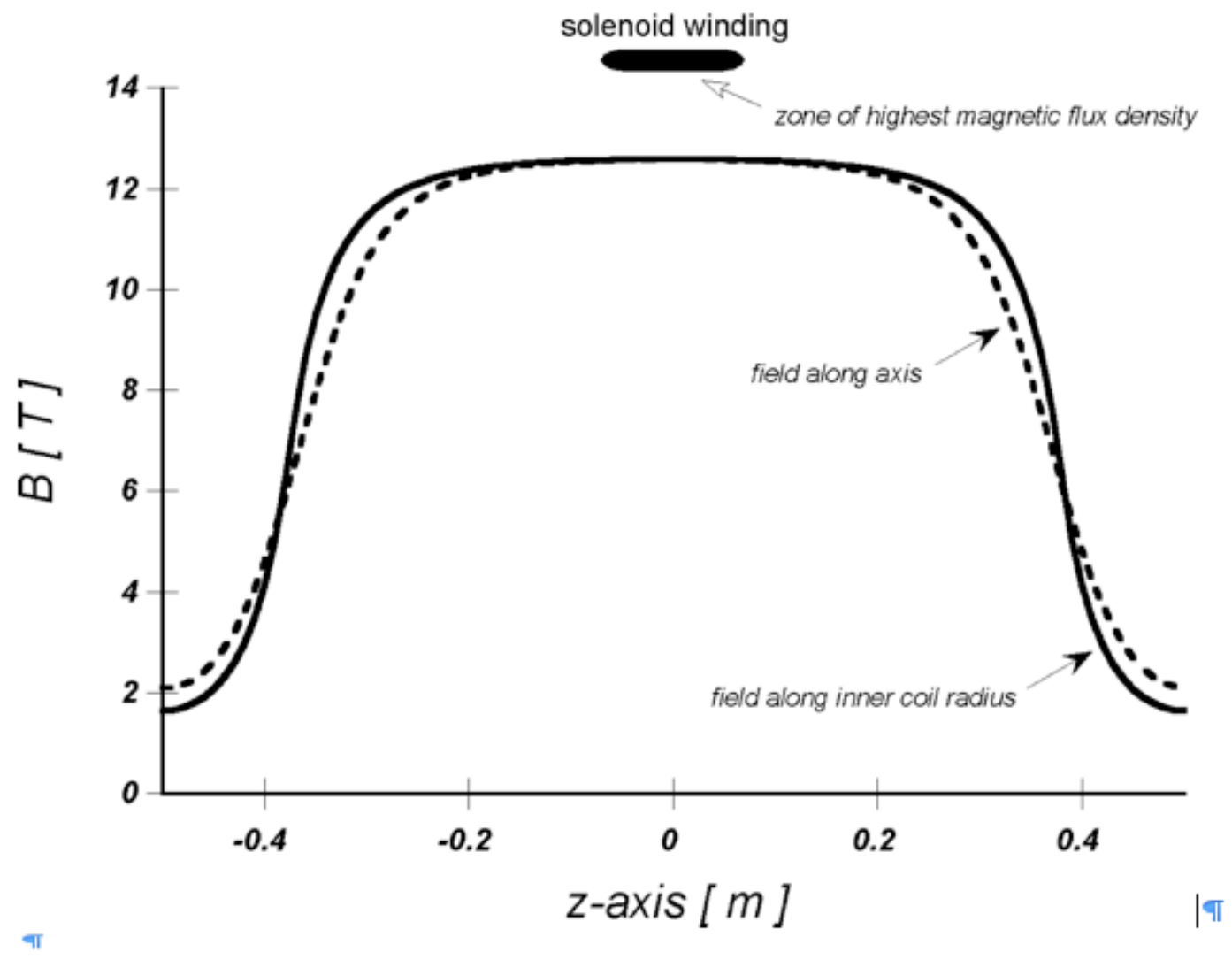

Figure 11: Comparison of $|\mathrm{B}|$ along the beam center axis and along the solenoid inner radius Indicated is the zone of highest magnetic field inside the solenoid winding.

Figure 11 shows $|\vec{B}|$ along the beam center axis and along the inner coil radius. The field nonuniformity must be included in ion optics simulations. Indicated in figure 11 is the location of the maximum magnetic field for a long solenoid. The solenoid current density must be below the "engineering" critical current density for the magnetic field encountered in that region.

Finite element analysis plays an important role in reliably predicting magnetic fields inside permeable materials. In the above design the iron region around the magnet winding is fully saturated (magnetic field $>2$ Tesla), as can be seen in figure 12. Plotted in figure 13 is the magnetic field versus radius (at $\mathrm{z}=0.0$, the middle of the solenoid, see figure 12) for different iron thicknesses. The magnetic field is constant within the solenoid bore, drops within the winding pack, and reverses its direction in the iron and the surrounding air. Since the iron is fully saturated it cannot completely shield the magnetic field return flux from the induction cells and the adjacent structures. With a $4 \mathrm{~cm}$ return iron yoke the air magnetic field at the location of the induction cells would be around 0.2 Tesla (see figure 14).

Increasing the iron thickness is the only option to further reduce the return field. That effect can be seen in figure 14. A significant thickness is required to shield a 12 
Tesla solenoid field from the induction cells. Currently it is not clear if total shielding is required to maintain the full flux swing inside the induction cells since the induction cell magnetization is perpendicular to the solenoid magnetization direction. In addition, the induction cell magnetization is a transient effect compared to the DC magnetization caused by the solenoid.

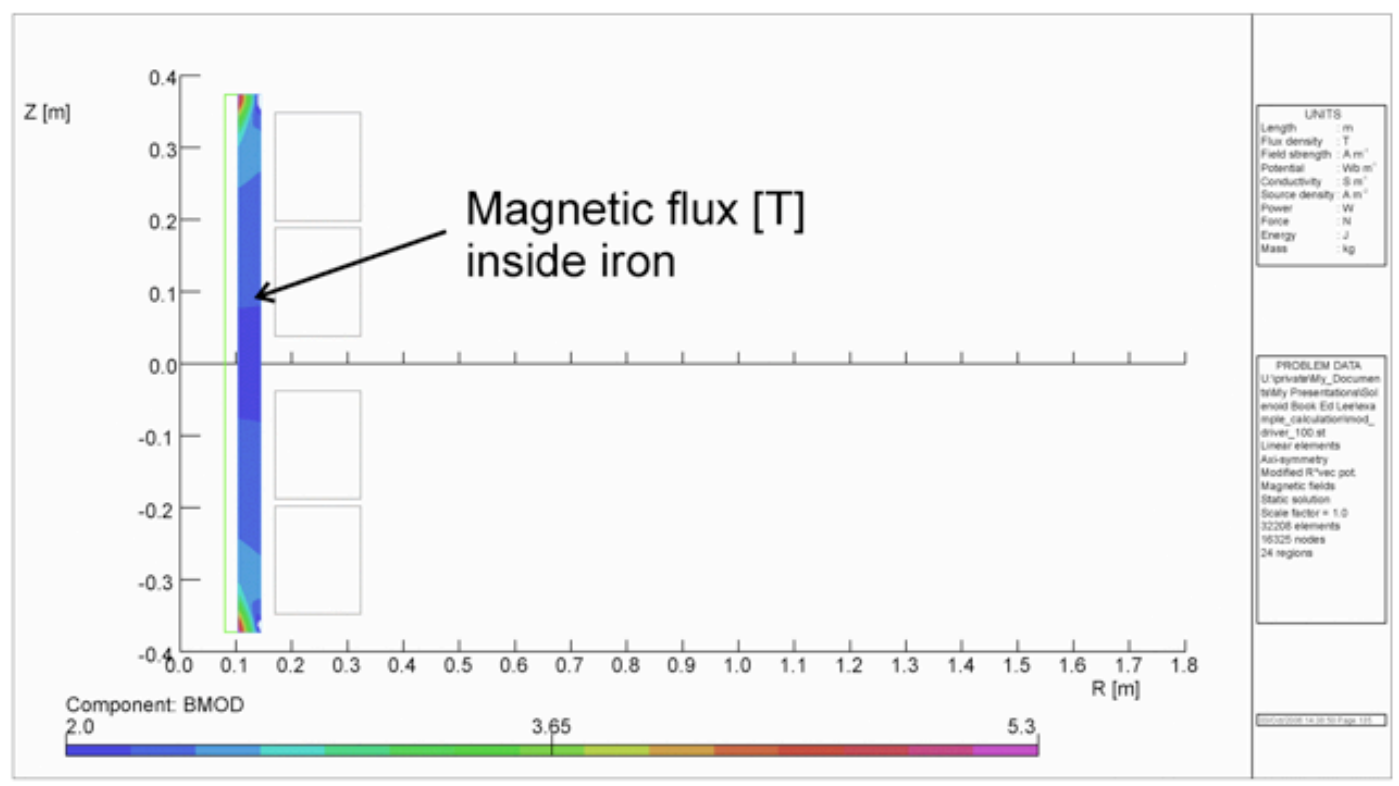

Figure 12: The solenoid iron return yoke is fully saturated.

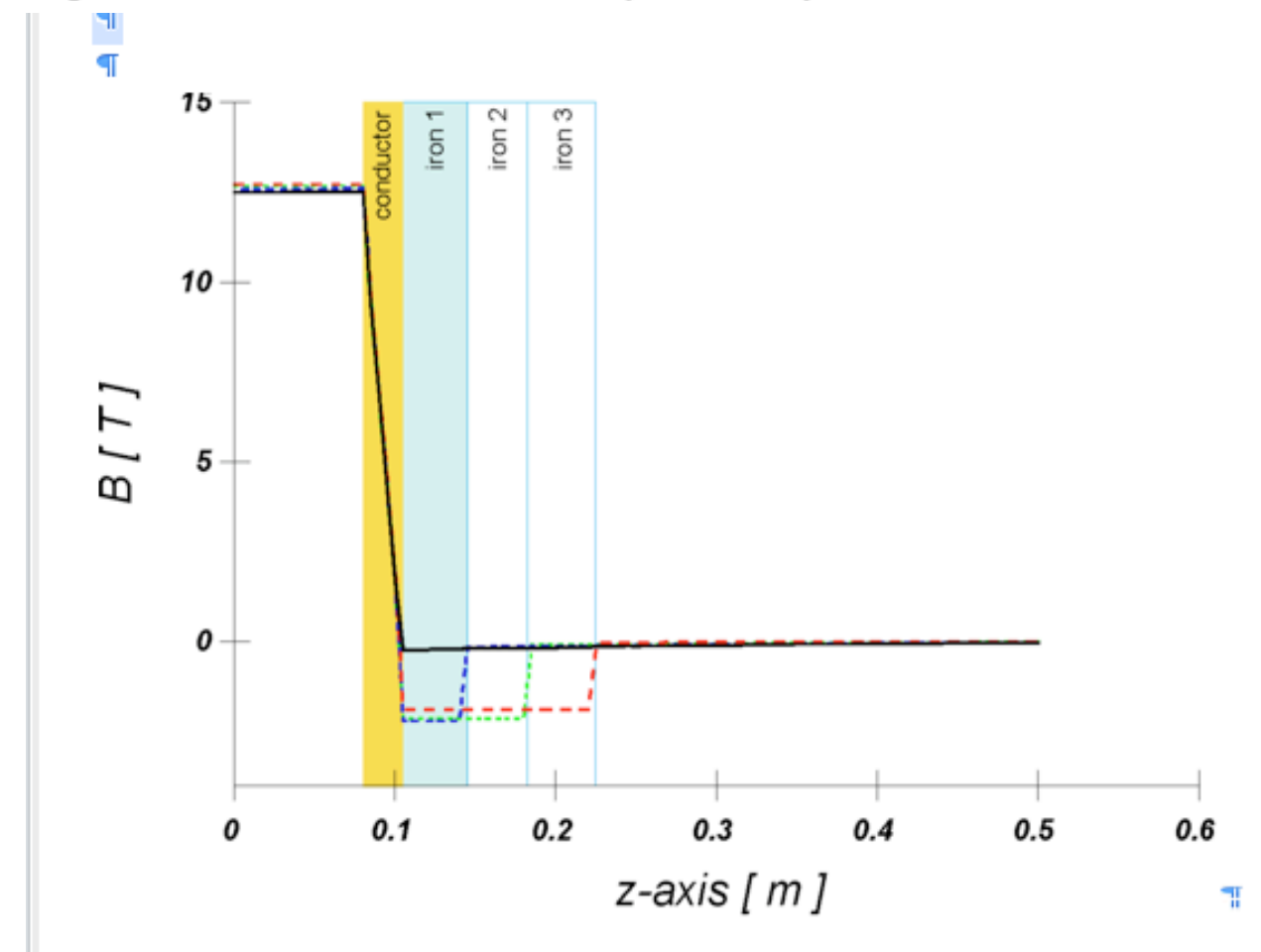

Figure 13: Magnetic field (in solenoid center) versus radius for different iron thicknesses. 


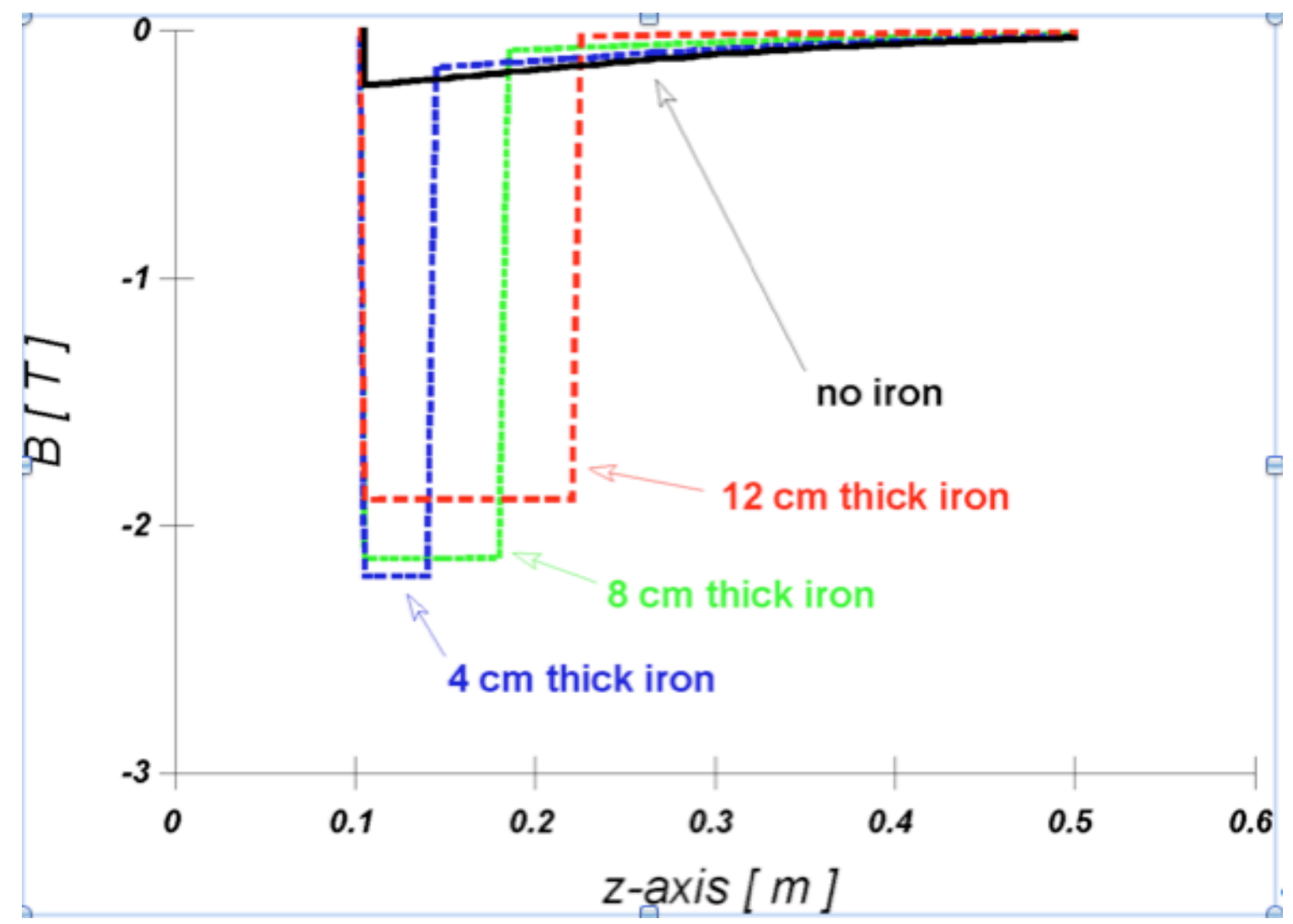

Figure 14: Solenoid return field in the vicinity of the induction cells for different iron return yoke thicknesses.

\section{References}

20-1 B. Grant Logan: "What product might a renewal of Heavy Ion Fusion development offer that competes with methane microbes and hydrogen HTGRs?” internal HIF-VNL report, May 1, 2006, LBNL-61905.

20-2 Applied Superconductivity Center, University of Wisconsin-Madison, http://www.asc.wisc.edu/ or http://magnet.fsu.edu/ lee/plot/plot.htm\}

\section{Biot-Savart Formula}

The Biot-Savart formula is usually an integration along a wire path, and it is therefore an attractive tool when the path is mathematically specified and there are no permeable materials. It may be used with a CAD program that lays out the wire path, which in general is a vector position $\vec{R}(s)$, where $s$ is path length measured along the wire. This is a good way to compute the fields of magnet leads and other deviations from ideal solenoidal symmetry. The Biot-Savart formula is often presented in texts as an experimental result for a straight wire and then generalized for an arbitrary wire path. 
Here we briefly derive it from the basic magnetostatic eqns. (2.1a,b). It is then used to obtain the fields of a straight wire segment of finite length and of a helix.

\subsection{General formula}

In the absence of magnetic materials the Cartesian components of a static magnetic field satisfy

$$
\nabla^{2} \vec{B}=\vec{\nabla} \vec{\nabla} \cdot \vec{B}-\vec{\nabla} x(\vec{\nabla} x \vec{B})=-\mu_{0} \vec{\nabla} x \vec{J}
$$

so the Green function solution is

$$
\vec{B}=\frac{\mu_{0}}{4 \pi} \int d^{3} r^{\prime} \frac{\vec{\nabla}^{\prime} x \vec{J}^{\prime}}{\left|\vec{r}-\vec{r}^{\prime}\right|}
$$

An integration by parts gives the most general form of the Biot-Savart law:

$$
\begin{aligned}
& \vec{B}=\frac{\mu_{0}}{4 \pi} \int d^{3} r^{\prime} \vec{J}^{\prime} x \vec{\nabla}^{\prime} \frac{1}{\left|\vec{r}-\vec{r}^{\prime}\right|} \\
& =\frac{\mu_{0}}{4 \pi} \int d^{3} r^{\prime} \vec{J}^{\prime} x \frac{\left(\vec{r}-\vec{r}^{\prime}\right)}{\left|\vec{r}-\vec{r}^{\prime}\right|^{3}} .
\end{aligned}
$$

If $\vec{J}^{\text {mag }}$ is known it can be added to $\vec{J}$ in eqn (21.3).

For a wire (of infinitesimal thickness) with path $\vec{R}(s)$ and carrying current I, eqn. (21.3) may be written as

$$
\vec{B}=\frac{\mu_{0} I}{4 \pi} \int d s \hat{e}(s) x \frac{(\vec{r}-\vec{R}(s))}{|\vec{r}-\vec{R}(s)|^{3}},
$$

where $\hat{e}(s)$ is the unit vector along the wire in the direction of the current:

$$
\hat{e}(s)=\frac{d \vec{R}(s)}{d s} .
$$


If the field point $\vec{r}$ is not close to the wire, eqn. (21.4) may be approximated by a finite sum. Divide a closed circuit into $N$ segments of length $L_{i}$ with $\overrightarrow{R_{i}}$ and $\vec{e}_{i}$ the position and tangent unit vector at the ith segment center. Then we have

$$
\vec{B}(\vec{r}) \approx \frac{\mu_{0} I}{4 \pi} \sum_{i=1}^{N} L_{i} \hat{e}_{i} x \frac{\vec{r}-\vec{R}_{i}}{\left|\vec{r}-\vec{R}_{i}\right|^{3}}
$$

This formula gives the correct on-axis field for a simple current loop of radius $R$ (see eqn. (4.14) if $L_{i}=2 \pi R / N$ and $N>1$. However, it is no good for $\vec{r}$ close to $\vec{R}_{i}$. Often a current path is well represented by a series of straight wire segments of finite length, and the much more accurate formula given below can be used.

\subsection{Straight wire segment field}

First we recover the elementary Biot-Savart law for an infinitely long wire along the z- axis. In this case we have simply

$$
\hat{e}(s)=\hat{e}_{z}, \vec{R}(s)=s \hat{e}_{z} .
$$

Eqn. (21.4) becomes

$$
\vec{B}=\frac{\mu_{0} I}{4 \pi} \hat{e}_{z} x \vec{r} \int_{-\infty}^{+\infty} d s \frac{1}{{\sqrt{r^{2}+(z-s)^{2}}}^{3}}
$$

where as usual $r^{2}=x^{2}+y^{2}$. The integration is elementary in this case:

$$
\vec{B}=\left.\frac{\mu_{0} I}{4 \pi} \frac{\hat{e}_{z} x \vec{r}}{r^{2}} \frac{(s-z)}{\sqrt{r^{2}+(s-z)^{2}}}\right|_{-\infty} ^{+\infty}=\frac{\mu_{0} I}{2 \pi} \frac{\hat{e}_{z} x \vec{r}}{r^{2}}=\frac{\mu_{0} I}{2 \pi r} \hat{e}_{\theta}
$$

For a semi-infinite wire running from $-\infty$ to 0 we have

$$
\vec{B}=\frac{\mu_{0} I}{4 \pi} \frac{\hat{e}_{\theta}}{r}\left(1-\frac{z}{\sqrt{r^{2}+z^{2}}}\right) \text {. }
$$

However there must be an additional current path (unspecified here) leading away from the wire end at $z=0$ to avoid a non-zero divergence of $\vec{J}$. Interestingly, eqn. (21.10) is the exact field for the wire current terminating at $z=0$ plus a spherically symmetric current $(I)$ or equivalent displacement current flowing outwards from the termination point. In either case Maxwell's equations are satisfied. 
A generalization of the above example can be derived for a straight wire segment between arbitrary points $\vec{r}_{1}$ and $\vec{r}_{2}$; we define

$$
\begin{aligned}
& \vec{C}=\frac{\vec{r}_{1}+\vec{r}_{2}}{2}=\text { segment center, } \\
& L=\left|\vec{r}_{2}-\vec{r}_{1}\right|=\text { segment length, } \\
& \hat{e}=\frac{\vec{r}_{2}-\vec{r}_{1}}{L}=\text { segment direction. }
\end{aligned}
$$

Then the wire path is

$$
\vec{R}(s)=\vec{C}+s \hat{e}
$$

with $-L / 2<s<L / 2$, and eqn (21.4) becomes

$$
\vec{B}=\frac{\mu_{0} I}{4 \pi} \int_{-L / 2}^{L / 2} d s \hat{e} x \frac{[\vec{r}-(\vec{C}+s \hat{e})]}{|\vec{r}-(\vec{C}+s \hat{e})|^{3}}
$$

The integration is again elementary, but it helps to employ the definitions

$$
\alpha=\hat{e} \cdot(\vec{r}-\vec{C}), \vec{\beta}=\hat{e} x(\vec{r}-\vec{C}),
$$

with $|\vec{r}-\vec{C}|^{2}=\alpha^{2}+|\vec{\beta}|^{2}$. Making the change of variable $t=s-\alpha$, we get from eqn. (21.12)

$$
\begin{aligned}
& \vec{B}=\left.\frac{\mu_{0} I}{4 \pi} \frac{\vec{\beta}}{|\vec{\beta}|^{2}} \frac{t}{\sqrt{|\vec{\beta}|^{2}+t^{2}}}\right|_{-L / 2-\alpha} ^{L / 2-\alpha} \\
& =\frac{\mu_{0} I}{4 \pi} \frac{\vec{\beta}}{|\vec{\beta}|^{2}}\left[\frac{(L / 2-\alpha)}{\sqrt{|\vec{\beta}|^{2}+(L / 2-\alpha)^{2}}}+\frac{(L / 2+\alpha)}{\sqrt{|\vec{\beta}|^{2}+(L / 2+\alpha)^{2}}}\right] .
\end{aligned}
$$

This formula may be used for the field from a straight magnet lead provided the coil field is computed with similar detail to avoid $\vec{\nabla} \cdot \vec{J} \neq 0$. Also, the field of a complete circuit with curved wired sections can be computed as a sum of fields from short straight sections, each with specified endpoints and defined quantities $(\vec{C}, L, \hat{e}, \alpha, \vec{\beta})$. 


\subsection{Helix field}

The path representation of a helix is more complicated; $\rho$ is its radius (wire distance from $z$-axis), $p$ is the helix pitch angle (restricted to $0<p<\pi / 2$ ), and $\tau$ is a variable proportional to $s$ such that $0 \leq \tau \leq 2 \pi$ gives one loop. Then we may write

$$
R_{x}=\rho \cos (\tau), R_{y}=\rho \sin (\tau), R_{z}=\rho \tau \tan (p) .
$$

We have $(d s)^{2}=|d \vec{R}|^{2}=\rho^{2}\left[1+\tan ^{2}(p)\right](d \tau)^{2}$, so

$$
\tau=\frac{s}{\rho \sqrt{1+\tan ^{2}(p)}}=\frac{s \cos (p)}{\rho},
$$

and $R_{z}=s \sin (p)$, as expected from the definition of helix pitch.

The helix period length is $2 \pi \rho \tan (p)=1 / N$, where $N$ is the number of turns per meter, so the mean $\theta$-component of surface current density is

$$
\langle S\rangle=N I,
$$

while the total z-current equals I (no return helix is assumed).

For an infinitely long helix, the on-axis $z$ component of the field is by symmetry,

$$
B_{0}(z)=\mu_{0}\langle S\rangle=\mu_{0} N I
$$

For $r>>$ we also have

$$
B_{\theta} \rightarrow \frac{\mu_{0} I}{2 \pi r}
$$

Close to the wire, which has infinitesimal thickness, the field diverges strongly. The onaxis transverse field does not vanish, but has a constant absolute value with direction rotating $90^{\circ}$ out of phase from the wire path. It is an interesting exercise to visualize the field lines.

To proceed with the helix field calculation, first note that 


$$
\begin{aligned}
& \hat{e}(s)=\frac{d \vec{R}}{d s}=\frac{d \tau}{d s} \frac{d \vec{R}}{d \tau}=\cos (p)\left[-\hat{e}_{x} \sin (\tau)+\hat{e}_{y} \cos (\tau)+\hat{e}_{z} \tan (p)\right], \\
& \hat{e}(s) x(\vec{r}-\vec{R}(s))=\cos (p)\left\{\hat{e}_{x}\left[\left(z-R_{z}\right) \cos (\tau)-\left(y-R_{y}\right) \tan (p)\right]\right. \\
& \left.+\hat{e}_{y}\left[\left(x-R_{x}\right) \tan (p)+\left(z-R_{z}\right) \sin (\tau)\right]-\hat{e}_{z}\left[\left(y-R_{y}\right) \sin (\tau)+\left(x-R_{x}\right) \cos (\tau)\right]\right\} .
\end{aligned}
$$

Each Cartesian field component may be projected from eqn. (21.4); for example

$$
B_{z}=\vec{B} \cdot \hat{e}_{z}=-\frac{\mu_{0} I}{4 \pi} \int d s \frac{\cos (p)\left[\left(y-R_{y}\right) \sin (\tau)+\left(x-R_{x}\right) \cos (\tau)\right]}{\sqrt{\left(x-R_{x}\right)^{2}+\left(y-R_{y}\right)^{2}+\left(z-R_{z}\right)^{2}}} .
$$

This may be evaluated by a numerical integration package inserting the given definitions of $\vec{R}(s)$. It helps to use $\tau=s \cos (p) / \rho$ as the integration variable. A helix might have, for example, $\mathrm{m}$ periods with $0 \leq \tau \leq 2 \pi m$ and longitudinal dimension running from $\mathrm{z}=0$ to $\mathrm{z}=2 \pi m \rho \tan (p)$. The components $B_{x}$ and $B_{y}$ are found in similar fashion.

For an infinite helix the on-axis longitudinal field is

$$
\begin{aligned}
& B_{z o}=\frac{\mu_{0} I}{4 \pi} \cos (p) \int_{-\infty}^{+\infty} d s \frac{\left[R_{y} \sin (\tau)+R_{x} \cos (\tau)\right]}{{\sqrt{R_{x}^{2}+R_{y}^{2}+\left(z-R_{z}\right)^{2}}}^{3}} \\
& =\frac{\mu_{0} I}{4 \pi} \cos (p) \int_{-\infty}^{+\infty} \frac{d u}{\sin (p)} \frac{\rho}{{\sqrt{\rho^{2}+u^{2}}}^{3}},
\end{aligned}
$$

where $u=R_{z}-z=s \sin (p)-z$. The integration is again elementary:

$$
B_{z_{o}}=\left.\frac{\mu_{0} I \rho}{4 \pi \tan (p)} \frac{1}{\rho^{2}} \frac{u}{\sqrt{\rho^{2}+u^{2}}}\right|_{-\infty} ^{+\infty}=\frac{\mu_{0} I}{2 \pi \rho \tan (p)}=\mu_{0} N I,
$$

as advertised in eqn. (21.18).

The transverse fields of a finite or infinite helix are

$$
B_{x}=\vec{B} \cdot \hat{e}_{x}=\frac{\mu_{0} I}{4 \pi} \int d s \frac{\cos (p)\left[\left(z-R_{z}\right) \cos (\tau)-\left(y-R_{y}\right) \tan (p)\right]}{\sqrt{\left(x-R_{x}\right)^{2}+\left(y-R_{y}\right)^{2}+\left(z-R_{z}\right)^{2}}}
$$




$$
B_{y}=\vec{B} \cdot \hat{e}_{y}=\frac{\mu_{0} I}{4 \pi} \int d s \frac{\cos (p)\left[\left(x-R_{x}\right) \tan (p)+\left(z-R_{z}\right) \sin (\tau)\right]}{\sqrt{\left(x-R_{x}\right)^{2}+\left(y-R_{y}\right)^{2}+\left(z-R_{z}\right)^{2}}}
$$

These components may also be evaluated numerically using $\tau$ as the variable of integration.

The on-axis transverse fields for an infinite helix are

$$
\begin{aligned}
& B_{x o}=\frac{\mu_{0} I \cos (p)}{4 \pi} \int_{-\infty}^{+\infty} d s \frac{[\rho \sin (\tau) \tan (p)+(z-\tau \rho \tan (p)) \cos (\tau)]}{{\sqrt{\rho^{2}+(z-\tau \rho \tan (p))^{2}}}^{3}}, \\
& B_{y o}=\frac{\mu_{0} I \cos (p)}{4 \pi} \int_{-\infty}^{+\infty} d s \frac{[(z-\tau \rho \tan (p)) \sin (\tau)-\rho \cos (\tau) \tan (p)]}{{\sqrt{\rho^{2}+(z-\tau \rho \tan (p))^{2}}}^{3}} .
\end{aligned}
$$

Making the change of variable $u=R_{z}-z=s \sin (p)-z$ we get

$$
\begin{aligned}
& B_{x o}=\frac{\mu_{0} I}{4 \pi \tan (p)} \int_{-\infty}^{+\infty} d u \frac{\left[\rho \tan (p) \sin \left(\frac{u+z}{\rho \tan (p)}\right)-u \cos \left(\frac{u+z}{\rho \tan (p)}\right)\right]}{{\sqrt{\rho^{2}+u^{2}}}^{3}}, \\
& B_{y o}=\frac{-\mu_{0} I}{4 \pi \tan (p)} \int_{-\infty}^{+\infty} d u \frac{\left[u \sin \left(\frac{u+z}{\rho \tan (p)}\right)+\rho \tan (p) \cos \left(\frac{u+z}{\rho \tan (p)}\right)\right]}{{\sqrt{\rho^{2}+u^{2}}}^{3}} .
\end{aligned}
$$

It is seen that $B_{x_{0}}$ vanishes at $z=0, \pm \pi \rho \tan (p), \pm 2 \pi \rho \tan (p), \ldots$, consistent with the helical symmetry. At the coordinate origin we have, setting $u=\rho v$,

$$
B_{y_{0}}(0)=-\frac{\mu_{0} I}{2 \pi \rho \tan (p)} \int_{0}^{\infty} d v\left[\frac{v \sin \left(\frac{v}{\tan (p)}\right)}{{\sqrt{1+v^{2}}}^{3}}+\frac{\tan (p) \cos \left(\frac{v}{\tan (p)}\right)}{{\sqrt{1+v^{2}}}^{3}}\right]
$$




$$
=-\frac{\mu_{0} I}{2 \pi \rho \tan (p)}\left[\frac{\mathrm{K}_{0}(1 / \tan (p))}{\tan (p)}+\mathrm{K}_{1}(1 / \tan (p))\right],
$$

where $\mathrm{K}_{0}$ and $\mathrm{K}_{1}$ are the modified Bessel functions. Setting $1 / \tan (p)=2 \pi \rho N$ we have

$$
B_{y_{0}}(0)=-\mu_{0} I N\left[(2 \pi \rho N) \mathrm{K}_{0}(2 \pi \rho N)+\mathrm{K}_{1}(2 \pi \rho N)\right] .
$$

Periodicity yields

$$
\vec{B}_{\perp_{0}}=B_{y_{0}}(0)\left[\begin{array}{lll}
\hat{e}_{y} & \cos (\tau)-\hat{e}_{x} & \sin (\tau)
\end{array}\right] .
$$

Generally the parameter $2 \pi \rho N$ is much larger that unity, and the Bessel functions may be expanded; the leading terms give

$$
B_{y_{0}}(0) \approx-\left(\mu_{0} I N\right) \sqrt{\frac{\pi}{2}}\left(\sqrt{2 \pi \rho N}+\frac{7}{8 \sqrt{2 \pi \rho N}}\right) e^{-2 \pi \rho N},
$$

which is usually very small. For a concrete example set $(N=20 \operatorname{turn} / \mathrm{m}, \rho=.1 \mathrm{~m})$; then $2 \pi \rho N=12.566$, and

$$
\frac{B_{y_{0}}(0)}{-\mu_{0} I N} \approx \sqrt{\frac{\pi}{2}}\left(\sqrt{12.566}+\frac{7}{8 \sqrt{12.566}}\right) e^{-12.566} \cong .000017 .
$$

\section{Single Particle Equations of Motion in an Arbitrary Electromagnetic Field}

Although the topic of this report is the calculation of fields, we also include this brief section on the particle equations of motion. There is no assumption of solenoidal symmetry or constancy of fields in time. The goal is to have a convenient reference where these equations are written using longitudinal distance $z$ as the independent variable instead of time $t$. This form displays the geometric aberrations, which are mixed with field aberrations and are hidden in the compact time-dependent formulation.

\subsection{Time as the independent variable}

With time as the independent variable, an ion's velocity $\vec{v}$ satisfies the Lorentz equation of motion

$$
\frac{d(\gamma \vec{v})}{d t}=\frac{q e}{M}(\vec{E}+\vec{v} \times \vec{B})
$$

with relativistic factor 


$$
\gamma=\left(1-v^{2} / c^{2}\right)^{-1 / 2}=\left(1+\gamma^{2} v^{2} / c^{2}\right)^{1 / 2}
$$

Here $q$ is the ion's charge state, $M$ is its mass, $v=|\vec{v}|$, and we use the natural constants

$$
e=1.6022 \ldots x 10^{-19} C, c=2.9979 \ldots x 10^{8} \mathrm{~m} / \mathrm{s} .
$$

The electric and magnetic fields, $\vec{E}(\vec{r}, t)$ and $\vec{B}(\vec{r}, t)$, are assumed to be known or are computable.

\subsection{Longitudinal distance as the independent variable}

For time-dependent, multi-particle simulations it is convenient to use eqn. (22.1) as it stands, or with $\gamma=1$ for non-relativistic cases. However there are situations where it is preferable to use the longitudinal position $z$ as the independent variable. One example is the simulation of an ion source operating in steady state. In principle we may always use $z$ instead of $t$ if there is no turning back of ion orbits. In addition to the particle variables $\left\{x(z), y(z), v_{x}(z), v_{y}(z), v_{z}(z), v(z), \gamma(z)\right\}$ we also calculate $t(z)$, the time when an ion is at $z$. The fields the ion experiences are then

$$
\vec{B}=\vec{B}(\vec{r}(z), t(z)), \quad \vec{E}=\vec{E}(\vec{r}(z), t(z)) .
$$

The time derivative in eqn. (22.1) is converted to a $z$-derivative by the relation

$$
\frac{d}{d t}=v_{z} \frac{d}{d z}
$$

Derivatives with respect to $z$ are denoted here by a "prime", i.e. $x^{\prime}(z)=d x / d z$, etc. Then we get

$$
\begin{aligned}
& v_{x}=\frac{d x}{d t}=v_{z} \frac{d x}{d z}=v_{z} x^{\prime}, \\
& v_{y}=\frac{d y}{d t}=v_{z} \frac{d y}{d z}=v_{z} y^{\prime} .
\end{aligned}
$$

The absolute velocity is

$$
v(z)=\sqrt{v_{x}^{2}+v_{y}^{2}+v_{z}^{2}}
$$

so substituting from eqn. (22.5) (assuming $\left.v_{z}>0\right)$, 


$$
v_{z}=\frac{v(z)}{\sqrt{1+x^{\prime 2}+y^{\prime 2}}} .
$$

The time derivative may therefore be written

$$
\frac{d}{d t}=\frac{v(z)}{\sqrt{1+x^{\prime 2}+y^{\prime 2}}} \frac{d}{d z} .
$$

The factor $\sqrt{1+x^{\prime 2}+y^{\prime 2}}$ in eqns. $(22.7,22.8)$, which we will denote by $\sqrt{\ldots}$, is the cause of the geometric aberrations during transport close to the $z$ axis.

Eliminating time, eqn. (22.1) yields

$$
\begin{aligned}
& \frac{v}{\sqrt{\ldots}} \frac{d}{d z} \frac{\gamma v}{\sqrt{\ldots}} \frac{d x}{d z}=\frac{q e}{M}\left(E_{x}+\frac{v y^{\prime}}{\sqrt{\ldots}} B_{z}-\frac{v}{\sqrt{\ldots}} B_{y}\right), \\
& \frac{v}{\sqrt{\ldots}} \frac{d}{d z} \frac{\gamma v}{\sqrt{\ldots}} \frac{d y}{d z}=\frac{q e}{M}\left(E_{y}+\frac{v}{\sqrt{\ldots}} B_{x}-\frac{v x^{\prime}}{\sqrt{\ldots}} B_{z}\right) .
\end{aligned}
$$

We also need an equation for $\gamma$ (or equivalently $v$ or $\gamma v$ ). From eqns. (22.1) and (22.2) we derive

$$
\frac{d}{d t} \gamma=\frac{q e}{M c^{2}} \vec{v} \cdot \vec{E}
$$

which becomes

$$
\frac{d \gamma}{d z}=\frac{q e}{M c^{2}}\left(x^{\prime} E_{x}+y^{\prime} E_{y}+E_{z}\right)
$$

or equivalently

$$
\begin{aligned}
& \frac{d v}{d z}=\frac{q e}{M} \frac{1}{\gamma^{3} v}\left(x^{\prime} E_{x}+y^{\prime} E_{y}+E_{z}\right), \\
& \frac{d \gamma v}{d z}=\frac{q e}{M} \frac{1}{v}\left(x^{\prime} E_{x}+y^{\prime} E_{y}+E_{z}\right) .
\end{aligned}
$$

The equation for time is simply

$$
\frac{d t}{d z}=\frac{1}{v_{z}}=\frac{\sqrt{\ldots}}{v} .
$$


Eqns. (22.9a,b) are of an awkward form since, due to the differentiation of $\sqrt{\ldots}$, the second derivatives $x^{\prime \prime}$ and $y^{\prime \prime}$ appear together. A simplification results when these equations are linearly combined to place these second derivatives in separate equations. It also helps to eliminate $d(\gamma v) / d z$ using eqn. (22.11c). These steps finally yield after some algebra:

$$
\begin{aligned}
& x^{\prime \prime}=\frac{q e}{M} \frac{1}{\gamma v^{2}}\left\{\sqrt{\ldots}^{2}\left(E_{x}-x^{\prime} E_{z}\right)+v \sqrt{\ldots}\left[x^{\prime} y^{\prime} B_{x}-\left(1+x^{\prime 2}\right) B_{y}+y^{\prime} B_{z}\right]\right\}, \\
& y^{\prime \prime}=\frac{q e}{M} \frac{1}{\gamma v^{2}}\left\{\sqrt{\ldots}^{2}\left(E_{y}-y^{\prime} E_{z}\right)+v \sqrt{\ldots}\left[-x^{\prime} y^{\prime} B_{y}+\left(1+y^{\prime 2}\right) B_{x}-x^{\prime} B_{z}\right]\right\} .
\end{aligned}
$$

Eqns. (22.11), (22.12), and (22.13) are valid as long as $d z / d t>0$; they contain all aberrations and may be used with tilted system axes and non-solenoidal fields.

\subsection{Paraxial approximation}

A simplified set of equations is usually used for calculations of beam transport along a straight axis $(z)$, in which small terms such as $x^{\prime 2}$ are dropped. This implies the fields are sufficiently weak, given the ion $s^{\prime}$ momenta, that trajectories are nearly parallel to the axis, ie $\left(x^{\prime}, y^{\prime}\right) \sim \varepsilon<<1$. If $2 \pi / k$ is the scale betatron wavelength, a formal ordering in $\varepsilon$ is

$$
(x, y) \sim \varepsilon / k,\left(x^{\prime \prime}, y^{\prime \prime}\right) \sim \varepsilon k,
$$

Often a simplified set of equations is used, in which terms such as $x^{\prime 2}$ are known to be small and are dropped. This is the "paraxial approximation", with formal ordering $d / d z \sim \varepsilon<1$ and $(x, y) \sim 1$ :

$$
\begin{aligned}
& \frac{q e}{M} \frac{1}{\gamma v^{2}}\left(E_{z}, v B_{z}\right) \sim k, \\
& \frac{q e}{M} \frac{1}{\gamma v^{2}}\left(E_{x}, E_{y}, v B_{x}, v B_{y}\right) \sim \varepsilon k .
\end{aligned}
$$

Dropping terms that are small of order $\varepsilon^{2}$ in eqn, (22.11-22.13) yields

$$
c^{2} \frac{d \gamma}{d z}=\gamma^{3} v \frac{d v}{d z}=v \frac{d \gamma v}{d z}=\frac{q e}{M} \mathrm{E}_{z}, \frac{d t}{d z}=\frac{1}{v}
$$




$$
\begin{aligned}
& x^{\prime \prime}=\frac{q e}{M} \frac{1}{\gamma v^{2}}\left[\left(\mathrm{E}_{x}-x^{\prime} \mathrm{E}_{z}\right)+v\left(y^{\prime} B_{z}-B_{y}\right)\right] \\
& y^{\prime \prime}=\frac{q e}{M} \frac{1}{\gamma v^{2}}\left[\left(\mathrm{E}_{y}-y^{\prime} \mathrm{E}_{z}\right)-v\left(x^{\prime} B_{z}-B_{x}\right)\right]
\end{aligned}
$$

In this approximation the geometric aberrations are gone but nonlinear terms representing field aberrations may still be present. If $(x, y)$ are small compared with the radii of wire layers or electrodes then the field expressions may be linearized and eqn. $(22.16,22.17)$ are free of aberrations except for the chromatic effects from spread momenta.

$\nabla^{2} B_{z}=-\mu_{0} \frac{1}{r} \frac{\partial}{\partial r} r J_{\theta}=-\frac{\mu_{0}}{r} \frac{\partial}{\partial r} r^{2} \frac{\partial K}{\partial r} \cos (\theta)$

\section{Steering Dipole Field}

Weak transverse dipole magnets that produce nearly uniform fields are used to steer and correct the beam orbit. Such a dipole winding is generally on a thin cylindrical shell (e.g. a printed circuit) around the beam pipe and may be combined with a solenoid. The longitudinal current density $J_{z}(r, \theta, z)$ has approximate $\sin (\theta)$ or $\cos (\theta)$ azimuthal dependence, with minor higher order transverse multipoles $(\sin (3 \theta), \sin (5 \theta)$, etc.) from the discrete wire layout. Non-linear fringe field components are produced by the magnet ends, where an azimuthal current density $J_{\theta}(r, \theta, z)$ completes the circuit.

We calculate the field from a current layer whose dominant Fourier components are

$$
J_{z}=K(r, z) \sin (\theta), \quad J_{\theta}=\frac{\partial K(r, z)}{\partial z} r \cos (\theta)
$$

with $J_{\theta}$ determined from $\vec{\nabla} \cdot \vec{J}=0$. This configuration produces an on-axis field $B_{x 0}(z)$, which deflects the beam in the $y$ direction. The approach used here is to first calculate $B_{z}$ from $J_{\theta}$ using the Green function for the Laplacian operator. Then the scalar potential $\phi$, which is valid in the magnet bore, is derived from $B_{z}$, and the complete field in the bore is given by $\vec{B}=-\mu_{0} \vec{\nabla} \phi$. Flux lines of $\vec{J}$ correspond to $K(r, z) \cos (\theta)=$ constant at fixed $r$. A widely used alternate approach is to calculate the vector potential component $A_{z}$ from $J_{z}$ (with gauge condition $\vec{\nabla}_{\perp} \cdot \vec{A}_{\perp}=0$ ) and use it to calculate the transverse magnetic field. This ignores the contribution from the transverse components of $\vec{A}$, a poor approximation if the current layer is not long compared with its radius.

A transverse dipole current density is not axisymmetric so we start with the general differential equation for $\vec{B}$, i.e. eqn. $(2.3 \mathrm{c})$. Its $z$ projection, with $J_{r}=0$ is 


$$
\nabla^{2} B_{z}=-\mu_{0} \frac{1}{r} \frac{\partial}{\partial r} r J_{\theta}=-\frac{\mu_{0}}{r} \frac{\partial}{\partial r} r^{2} \frac{\partial K}{\partial z} \cos (\theta)
$$

The Green function solution - see section 6 is

$$
B_{z}=\frac{\mu_{0}}{4 \pi} \int d^{3} r^{\prime} \frac{\left[\frac{1}{r^{\prime}} \frac{\partial}{\partial r^{\prime}} r^{\prime 2} \frac{\partial K\left(r^{\prime}, z^{\prime}\right) \cos \left(\theta^{\prime}\right)}{\partial z^{\prime}}\right]}{\left|\overrightarrow{r^{\prime}}-\vec{r}\right|} .
$$

Integrating by parts in $z^{\prime}$ and using $\partial\left|\overrightarrow{r^{\prime}}-\vec{r}\right| / \partial z^{\prime}=-\partial\left|\overrightarrow{r^{\prime}}-\vec{r}\right| / \partial r$, we get

$$
B_{z}=\frac{\partial}{\partial z} \frac{\mu_{0}}{4 \pi} \int \frac{d^{3} r^{\prime}\left[\frac{1}{r^{\prime}} \frac{\partial}{\partial r^{\prime}} r^{\prime 2} K\left(r^{\prime}, z^{\prime}\right) \cos \left(\theta^{\prime}\right)\right]}{\left|\overrightarrow{r^{\prime}}-\vec{r}\right|} .
$$

Since we also have $B_{z}=-\mu_{0} \partial \phi / \partial z$ in the vacuum bore, we may integrate (23.4) in $z$ to get

$$
\phi=-\frac{1}{4 \pi} \int \frac{d^{3} r^{\prime}\left[\frac{1}{r^{\prime}} \frac{\partial}{\partial r^{\prime}} r^{\prime 2} K\left(r^{\prime}, z^{\prime}\right) \cos \left(\theta^{\prime}\right)\right]}{\left|\overrightarrow{r^{\prime}}-\vec{r}\right|} .
$$

This is valid if the integration in $z$ does not pass through any part of a current layer. Inserting the identity $\cos \left(\theta^{\prime}\right)=\cos (\theta) \cos \left(\theta^{\prime}-\theta\right)-\sin \left(\theta^{\prime}\right) \sin \left(\theta^{\prime}-\theta\right)$, and displaying all variables, eqn. (23.5) becomes

$$
\begin{aligned}
\phi=-\frac{1}{2} \int_{-\infty}^{+\infty} d z^{\prime} \int_{0}^{\infty} d r^{\prime} r^{\prime}\left[\frac{1}{r^{\prime}} \frac{\partial}{\partial r^{\prime}} r^{\prime 2} K\left(r^{\prime}, z^{\prime}\right)\right] \\
\cdot \int_{0}^{2 \pi} \frac{d \theta^{\prime}}{2 \pi} \frac{\left[\cos (\theta) \cos \left(\theta^{\prime}-\theta\right)-\sin (\theta) \sin \left(\theta^{\prime}-\theta\right)\right]}{\sqrt{\left(z^{\prime}-z\right)^{2}+r^{\prime 2}+r^{2}-2 r^{\prime} r \cos \left(\theta^{\prime}-\theta\right)}},
\end{aligned}
$$

or in a more compact form:

$$
\phi=-\frac{\cos (\theta)}{2} \int_{-\infty}^{+\infty} d z^{\prime} \int_{0}^{\infty} d r^{\prime} \frac{\left[\frac{\partial}{\partial r^{\prime}} r^{\prime 2} K\left(r^{\prime}, z^{\prime}\right)\right] f_{1}(a)}{\sqrt{\left(z^{\prime}-z\right)^{2}+r^{\prime 2}+r^{2}}}
$$

with $f_{1}(a)$ as defined in section 7: 


$$
\begin{gathered}
f_{1}(a)=\int_{0}^{2 \pi} \frac{d \theta}{2 \pi} \frac{\cos (\theta)}{\sqrt{1-a \cos (\theta)}}=\frac{a}{4}\left(1+\frac{15}{32} a^{2}+\ldots\right), \\
a=\frac{2 r^{\prime} r}{\left(z^{\prime}-z\right)^{2}+r^{\prime 2}+r^{2}} .
\end{gathered}
$$

On the system axis $\phi=0$ but we may expand the integral solution, eqn. (23.7), for small $r$ to get the near-axis formula:

$$
\begin{aligned}
\phi^{\text {near axis }} & =g(z) r \cos (\theta)=g(z) x \\
g(z) & =-\frac{1}{4} \int_{-\infty}^{+\infty} d z^{\prime} \int_{0}^{\infty} d r^{\prime} r^{\prime} \frac{\frac{\partial}{\partial r^{\prime}} r^{\prime 2} K\left(r^{\prime}, z^{\prime}\right)}{{\sqrt{\left(z^{\prime}-z\right)^{2}+r^{\prime 2}}}^{3}} \\
& =\frac{1}{4} \int_{-\infty}^{+\infty} d z^{\prime} \int_{0}^{\infty} d r^{\prime} r^{\prime 2} K\left(r^{\prime}, z^{\prime}\right)\left(\frac{1}{{\sqrt{\left(z^{\prime}-z\right)^{2}+r^{\prime 2}}}^{3}}-\frac{3 r^{\prime 2}}{{\sqrt{\left(z^{\prime}-z\right)^{2}+r^{\prime 2}}}^{5}}\right) .
\end{aligned}
$$

The on-axis field is

$$
B_{x 0}=-\mu_{0} \frac{\partial \phi^{\text {near axis }}}{\partial \mathrm{x}}=-\mu_{0} g(z)
$$

A quasi-realistic model of a dipole current layer (radius $R$, length $\ell$ ) is

$$
J_{z}=K(r, z) \sin (\theta)=k \delta(r-R) \sin (\theta) \quad-\frac{\ell}{2}<z<\frac{\ell}{2} .
$$

Here $J_{\theta}$ is approximated by infinitesimally wide flows at $z= \pm \ell / 2$. Equation (23.10) yields

$$
\begin{aligned}
g(z) & =-\frac{k}{4}\left[\frac{2(z+\ell / 2)}{\sqrt{(z+\ell / 2)^{2}+R^{2}}}-\frac{2(z-\ell / 2)}{\sqrt{(z-\ell / 2)^{2}+R^{2}}}\right. \\
& \left.-\frac{(z+\ell / 2)^{3}}{{\sqrt{(z+\ell / 2)^{2}+R^{2}}}^{3}}+\frac{(z-\ell / 2)^{3}}{{\sqrt{(z-\ell / 2)^{2}+R^{2}}}^{3}}\right]
\end{aligned} .
$$

$B_{x o}(z)$ is plotted below for $\left(\mu_{0} k=1.0 T, \ell=.2 m, R=.1 m\right)$.

Off-axis fields are conveniently derived from the expansion of $\phi$ in powers of $r$. From $\nabla^{2} \phi=0$ we get: 


$$
\frac{1}{r} \frac{\partial}{\partial r} r \frac{\partial \phi}{\partial r}-\frac{\phi}{r^{2}}=-\frac{\partial^{2} \phi}{\partial z^{2}}
$$

The leading term is known to be $g(z) r \cos (\theta)=g x$. Iterating in eqn. (23.15) gives

$$
\begin{aligned}
\phi= & \cos (\theta)\left(g r-\frac{g^{\prime \prime} r^{3}}{8}+\frac{g^{i v} r^{5}}{192}-\ldots\right) \\
& =x\left[g-\frac{g^{\prime \prime}\left(x^{2}+y^{2}\right)}{8}+\frac{g^{i v}\left(x^{2}+y^{2}\right)^{2}}{192}-\ldots\right]
\end{aligned}
$$

It is easily shown from eqns (23.10 and 23.14$)$ that

$$
\int_{-\infty}^{+\infty} d z^{\prime} B_{x}(r, z)=\frac{\mu_{0}}{2} \int_{-\infty}^{+\infty} d z^{\prime} \int_{0}^{\infty} d r^{\prime} K\left(r^{\prime}, z^{\prime}\right)
$$

For the example distribution [eqn. (23.12)] this integral is simply $\mu_{0} k \ell / 2$.

We can actually define a global scalar potential $(\varphi)$ for the steering dipole potential as follows; let

$$
\vec{B}=\mu_{0} K(r, z) r \cos (\theta) \hat{e}_{r}+\vec{\nabla} \varphi
$$

This satisfies $\vec{\nabla} x \vec{B}=\mu_{0} \vec{J}$, and to make $\vec{\nabla} \cdot \vec{B}=0$ we require

$$
\nabla^{2} \varphi=-\frac{1}{r} \frac{\partial}{\partial r} r \mu_{0} K r \cos (\theta)
$$

The Green function solution is

$$
\varphi=\frac{\mu_{0}}{4 \pi} \int d^{3} r^{\prime} \frac{\frac{1}{r^{\prime}} \frac{\partial}{\partial r^{\prime}} r^{\prime 2} K^{\prime} \cos \left(\theta^{\prime}\right)}{\left|\overrightarrow{\mathrm{r}^{\prime}}-\overrightarrow{\mathrm{r}}\right|},
$$

and $B_{z}=\partial \varphi / \partial z$ is identical with eqn (23.4). It is apparent that in the magnet bore $\varphi=-\mu_{0} \phi$, but eqn (23.17) is also valid in the current layer. 


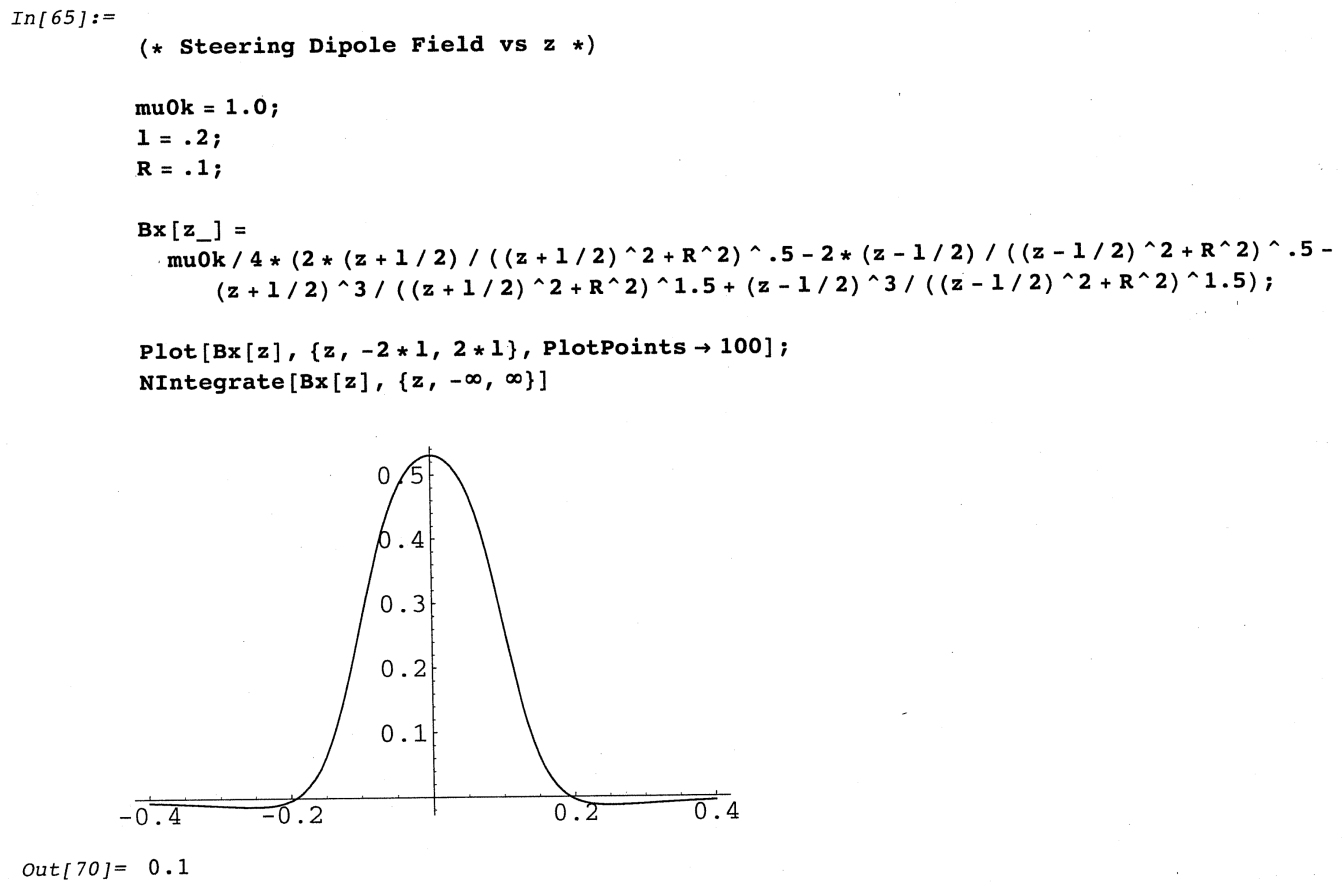

$\operatorname{out}[70]=0.1$ 